\title{
Multi-Phase Fracture-Matrix Interactions Under Stress Changes
}

\author{
Final Report
}

Reporting Period

September 21, 2001 - September 20, 2005

Principal Authors:

A. S. Grader, D. Elsworth, P. M. Halleck, F. Alvarao, A. Alajmi, Z. Karpyn, N. Mohammed, S. Al-Enezi

Report Issue Date: December 7, 2005

DOE Award Number: DE-FC26-01BC15355

Submitting Organization:

The Pennsylvania State University

The Energy Institute

A. S. Grader

203 Hosler Building

University Park, PA 16802 


\section{DISCLAIMER}

“This report was prepared as an account of work sponsored by an agency of the United States Government. Neither the United States Government nor any agency thereof, nor any of their employees, makes any warranty, express or implied, or assumes any legal liability or responsibility for the accuracy, completeness, or usefulness of any information, apparatus, product, or process disclosed, or represents that its use would not infringe privately owned rights. Reference herein to any specific commercial product, process, or service by trade name, trademark, manufacturer, or otherwise does not necessarily constitute or imply its endorsement, recommendation, or favoring by the United States Government or any agency thereof. The views and opinions of authors expressed herein do not necessarily state or reflect those of the United States Government or any agency thereof.” 


\begin{abstract}
The main objectives of this project are to quantify the changes in fracture porosity and multi-phase transport properties as a function of confining stress. These changes will be integrated into conceptual and numerical models that will improve our ability to predict and optimize fluid transport in fractured system. This report details our progress on: a. developing the direct experimental measurements of fracture aperture and topology and fluid occupancy using high-resolution x-ray micro-tomography, b. counter-current fluid transport between the matrix and the fracture, c. studying the effect of confining stress on the distribution of fracture aperture and two-phase flow, and $\mathbf{d}$. characterization of shear fractures and their impact on multi-phase flow.
\end{abstract}

The three-dimensional surface that describes the large-scale structure of the fracture in the porous medium can be determined using x-ray micro-tomography with significant accuracy. Several fractures have been scanned and the fracture aperture maps have been extracted. The success of the mapping of fracture aperture was followed by measuring the occupancy of the fracture by two immiscible phases, water and decane, and water and kerosene.

The distribution of fracture aperture depends on the effective confining stress, on the nature of the rock, and the type and distribution of the asperities that keep the fracture open. Fracture apertures at different confining stresses were obtained by micro-tomography covering a range of about two thousand psig. Initial analysis of the data shows a significant aperture closure with increase in effective confining stress. Visual and detailed descriptions of the process are shown in the report. Both extensional and shear fractures have been considered.

A series of water imbibition tests were conducted in which water was injected into a fracture and its migration into the matrix was monitored with CT and DR x-ray techniques. The objective was to 
understand the impact of the fracture, its topology and occupancy on the nature of mass transfer between the matrix and the fracture. Counter-current imbibition next to the fracture was observed and quantified, including the influence of formation layering.

A group of Shear fractures were studied, with layers perpendicular and parallel to t he main axis of the sample. The structures of the fractures as well as their impact on absolute permeability and on oil displacement by water were evaluated. Shear fractures perpendicular to the layers lead to a wide distribution of pores and to an overall increase in absolute permeability. Shear fractures parallel to the layers lead to an overall increase in absolute permeability, but a decrease in displacement efficiency.

This DoE project funded or partially funded three Ph.D. and four M.Sc. students at the Pennsylvania State University. The results from the research have yielded several abstracts, presentations and papers. Much of the work is still in the process of being published. 


\section{TABLE OF CONTENTS}

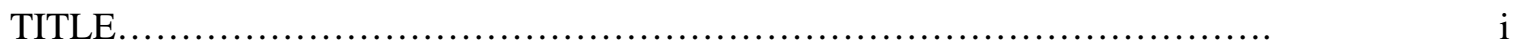

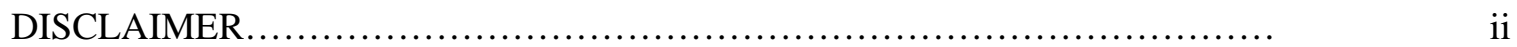

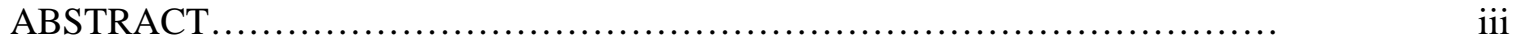

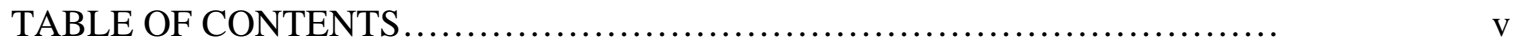

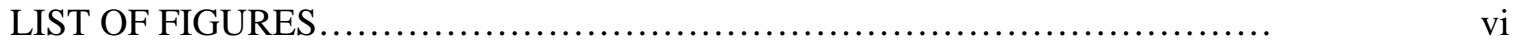

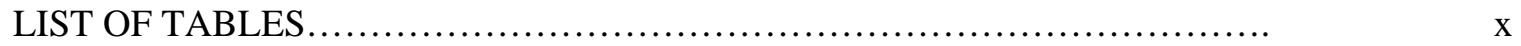

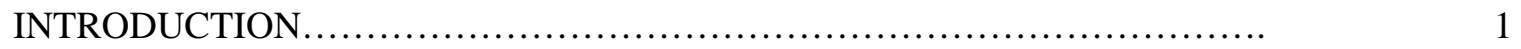

EXECUTIVE SUMMARY ............................................... 3

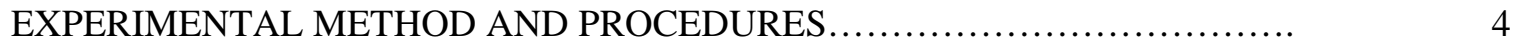

RESULTS AND DISCUSSION..............................................

Fracture Morphology and Aperture Distribution $r$

Matrix-Fracture interaction -Counter-Current Imbibition............ 19

Shear Fractures Parallel and Perpendicular to Bedding...................... 60

Effects of Confining Stress on Aperture Structure and Two-Phase Flow........ 76

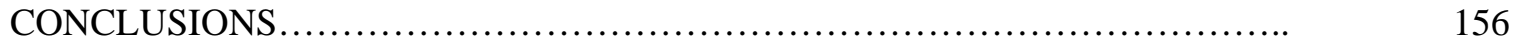

REFERENCES .......................................................... $\quad 156$

LIST OF ACRONYMS AND ABBREVIATIONS............................. 160 


\section{LIST OF FIGURES}

Figure 1: $\quad$ Fractured Berea sample and a detailed inset........................

Figure 2: A two-dimensional map of fracture aperture. Area: $16 \mathrm{~mm}$ x $30 \mathrm{~mm}$. Red: Contact regions. White: aperture of 1200 microns. Yellow: 600 microns contour

Figure 3: Fracture surrounded by matrix

Figure 4:

Three-dimensional images of the fracture under 500 and 2500 psig

confining pressure.

Figure 5:

Berea sandstone sample with fracture perpendicular to rock layering.

Figure 6:

Examples of original CT data (a) and connected fracture volume (b).

Image size had been cropped to 983x261 elements to reduce the computation effort

Figure 7: $\quad$ Examples of fracture and matrix regions used in correlating aperture with matrix porosity.

Figure 8: CT value as related to porosity as a function of fracture aperture....

Figure 9:

A representation of a portion of the fracture and adjacent matrix showing the matrix portion used in computing the adjacent porosity to the fracture.

Figure 10: Fracture aperture and adjacent porosity axial images.

Fracture aperture and adjacent porosity axial images showing the specific slices (20 - green, 140 - orange, 280 - red) and layers that are strongly correlated (black vertical lines).

Figure 12: A three-dimensional rendition of the fracture

A three-dimensional rendition of the connected volume of the fracture.

Figure 14:

A three-dimensional rendition of the fracture. Black spaces indicate asperities.

Figure 15:

Scanning sequence of experiment 2: capillary imbibition.

Schematic representation of capillary imbibition across fracturematrix interface..............................................

Figure 17: Digital Radiography of sample 2 initially saturated with oil...........

Figure 18: Cumulative water saturation during capillary imbibition obtained from digital radiography.

Figure 19: Incremental water saturation during capillary imbibition obtained from digital radiography.

Figure 20: Cumulative water saturation during capillary imbibition obtained from high-resolution CT scans.

Figure 21: Incremental water saturation during capillary imbibition obtained from high-resolution CT scans................................

Figure 22: Effect of rock bedding on spontaneous imbibition from subtracted CT scans.

Figure 23: Schematic representation of the CT section used for saturation profile calculation....

Figure 24: Cumulative water saturation profiles obtained from high-resolution 
CT scans. Horizontal orientation is $3 \mathrm{~mm}$ above the inlet end of the sample

Figure 25: Incremental water saturation profiles obtained from high-resolution CT scans. Horizontal orientation is $3 \mathrm{~mm}$ above the inlet end of the sample.

Figure 26: Changes in average water saturation as a function of time for a CT slice $3 \mathrm{~mm}$ above the bottom of the core.

Figure 27: Water saturation map at the end of capillary imbibition 1 showing fluid banks, $1 \mathrm{~mm}$ above the bottom of the core.

Figure 28: Water saturation map at the end of capillary imbibition 1 showing fluid banks and layering effect, $9 \mathrm{~mm}$ above the bottom of the core...

Figure 29: Schematic representation of experimental observations during capillary imbibition.

Figure 30: Grid model for two-dimensional imbibition Simulated water front using fracture capillary pressure equal to zero.. Simulated cumulative water front using similar capillary pressure curves in the fracture and the matrix.

Figure 35: Simulated cumulative water front obtained from two-dimensional two-phase imbibition model.

Figure 36:

Grid model for simulating three-dimensional imbibition...............

Water and oil flow vectors on axial cross-section at 4 and 90 minutes, slice $\mathrm{z}=11$

Figure 41: Water saturation changes in a coronal cross-section, slice $y=11 \ldots \ldots .$. and oil flow vectors on coronal cross-section at 4 and 90 minutes,

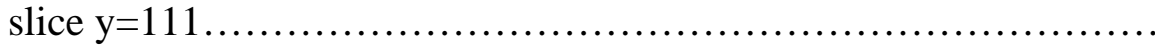

Figure 43: Water saturation changes in a sagital cross-section, slice $x=11 \ldots \ldots \ldots$ minutes, slice $\mathrm{x}=11$.

Figure 45: Qualitative comparison of cumulative water saturation profiles obtained from three-dimensional simulation and the experiment.......

Figure 46: Apparatus for fracturing in shear mode.

Figure 48: Three-dimensional renditions of a shear fracture in a sample cut perpendicular to bedding.

Figure 49: Experimental procedure for fluid flow experiment with shear fractures........................................................

Figure 50: Porosity distribution along the sample. The inlet side is on the right.

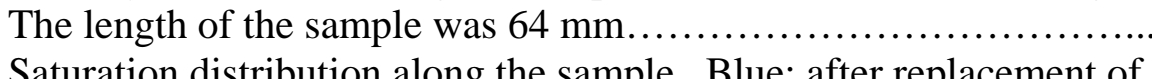


water with NaI-tagged water. Black: after an oil flood, at residual water saturation. Red: after a water flood, at residual oil saturation

Figure 52: Net oil recovery profile along the sample showing a region of relatively good sweep that corresponds to a region with poor fracture connectivity ...............................................

Figure 53: Three possible fracture conditions, of which the most likely one in the experiment is number three.....................................

Figure 54: An example of a sample with the bedding planes parallel to the main axis of the sample, before and after fracturing.

Figure 55: Binary maps of the fractures created in perpendicular and parallel to bedding planes samples. Left: Perpendicular to bedding. Right: Parallel to bedding........................................... 68

Figure 56: Schematic and actual layers dislocation after fracturing...............

Figure 57: Average water saturation for each slice at Swirr and Sor conditions.

Figure 58: Water saturation inside the fracture at Swirr condition

Figure 59: Oil saturation inside the fracture at Swirr condition. Dark gray is the fracture.

Figure 60: Both oil and water saturation at Swirr condition. Blue is water and red is oil...

Figure 61: A single CT slice at various stages of processing. a: original. b: rotated. c: cut and masked....

Figure 62:

Original core before and after scanning and extracting a region that contains the fracture.

CT image at the same location for increasing confining pressure

Figure 63: Slice 140 .

Figure 64:

Aperture profiles as a function of confining pressure at the same location in the sample.

Figure 65:

Aperture fracture map for 100, 500, 1000, 1500, 2000, and 2500 psig confining pressure.

Figure 66: a: CT profile at $x=140$. b: Images for 100 and 2500 psig confining pressure. c: CT profile at $\mathrm{x}=660$.

Figure 67: CT profiles at different locations (100 psig and 2500 psig confining

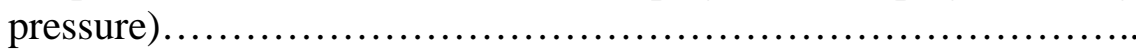

Figure 68: Fracture aperture surrounded by matrix.........................

Figure 69: Three-dimensional images of the fracture under 100 and 2500 psig confining pressures........................................ 86

Figure 70: $\quad$ Fracture aperture volume with coronal slice planes.................. 87

Figure 71: Cartoon of the three-dimensional (binary) fracture aperture array..... 87

Figure 72: Two dimensional fracture aperture maps. a: 100 psig. b: 2500 psig... 88

Figure 73: $\quad$ Two dimensional asperities maps............................. 90

Figure 74: Probability plot from the fracture aperture....................... 91

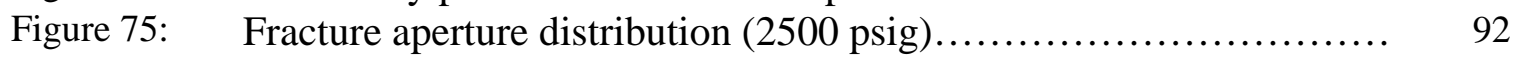

Figure 76: $\quad$ Fracture aperture distributions for 100 and 2500 psig............... 92

Figure 77: Three dimensional representation of fracture aperture at 2500 psig.... 94

Figure 78: $\quad$ Fracture aperture distribution 360 microns thickness or more.......... 95 


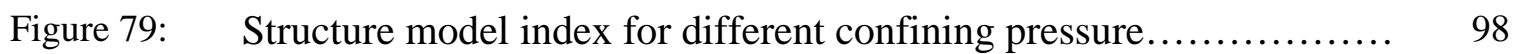

Figure 80: $\quad$ Fracture sub-division scheme into 4 blocks $(2 x 2 \ldots \ldots \ldots \ldots \ldots \ldots \ldots \ldots . . . \ldots 9$

Figure 81: $\quad$ Euler-Poincaré distribution for the 16x16 case at 500 psig............ 101

Figure 82: $\quad$ Euler-Poincaré distribution for the $16 \times 16$ case at 2500 psig.......... 102

Figure 83: $\quad$ SMI distribution for the $16 \times 16$ case at 2500 psig..................... 103

Figure 84: $\quad$ SMI distribution for the $16 \times 16$ case at 2500 psig. The width is 3.120 $\mathrm{mm}$ and the length is $3.968 \mathrm{~mm}$................................ 104

Figure 85: $\quad$ EPC distribution for the $2 \times 2,4 \times 4,8 \times 8$ and $16 \times 16$ case at 2500 psig... 105

Figure 86: $\quad$ EPC average values for the $2 \times 2,4 \times 4,8 \times 8$ and $16 \times 16$ case at 500 and 2500 psig................................................... 106

Figure 87: $\quad$ SMI average values for the $2 \times 2,4 \times 4,8 \times 8$ and $16 \times 16$ case at 500 and 2500 psig....................................................

Figure 88: Changes along pressure drop for different confining pressure at fixed injection rate................................................. 110

Figure 89: $\quad$ Pressure drop along the fracture for different fractional flow values... 111

Figure 90: $\quad$ Pressure drop along the fracture vs fractional flow, 1000 psig confining pressure.............................................. 112

Figure 91: $\quad$ Pressure drop in the fracture vs fractional flow for 800 and 1000 psig Figure 92: $\quad$ Pressure drop in the fracture vs fractional flow for 200 psig. Set \# 1 .. Figure 93: $\quad$ Pressure drop in the fracture vs fractional flow for 200 psig. Set \# 2.. Figure 94: $\quad$ Pressure drop in the fracture vs fractional flow for a confining pressure equal to 800 psig......................................

Figure 95: Fractional flow range: $0.25-0.50$. 800 psig confining pressure......... Figure 96: Fractional flow range: $0.50-0.83$. 800 psig confining pressure........

Figure 97: Pressure drop in the fracture vs fractional flow for 500 psig. Core \# 4

Figure 98: Fractured core (top), matrix reduction (bottom)...................

Figure 99:

Figure 100:

Figure 101:

Figure 102:

Figure 103: Pressure drop in the fracture for water injection.

Figure 104: Water pressure drop in the fracture for different saturation conditions Relative permeabilities curve for a fractured system at 500 psig....... Fracture volume for different threshold numbers.................... Three dimensional rendition of fracture asperities.

Fluids distribution maps: a: dry. b: oil. c: water at residual water saturation state.

Figure 105: Fluids distribution maps: a: dry. b: Residual oil. c: Water. imbibitions cycles.

Figure 107: Pressure drop in the fracture for different fractional flow. $\mathrm{q}_{\text {total }}=12$ $\mathrm{cc} / \mathrm{mim}$.

Figure 108: Fluids distribution in the fracture at low pressure drop. a: Oil b: water.......................................................

Figure 109: Oil saturation profile for the low pressure drop condition (left).Three-dimensional distribution of oil in the fracture (right)......

Figure 111: Oil bodies distribution at low pressure drop. 
spontaneous pressure change

Figure 113: Oil saturation profile for the high pressure drop condition

(left).Three-dimensional distribution of oil in the fracture (right).....

Figure 114: Oil saturation profile for the high pressure drop condition (left).Three-dimensional distribution of oil in the fracture (right)......

Figure 115: Oil distribution low pressure drop (a). Oil distribution high pressure drop (b) .........................................................

Figure 116: Pressure drop as a function of flow rate for a non-fractured and a fractured core

Figure 117: Schematic of the volume changes with confining pressure............. 142

Figure 118: CT profile in the matrix area near the fracture (Dry and Wet)........ 144

Figure 119: CT profile in the matrix area near the fracture at 500 and 2500 psig...

Figure 120:

Residual oil saturation map at 2500 psig.

Figure 121: Pressure drop in the fracture at 2500 psig for a total liquid flow rate of $1 \mathrm{cc} / \mathrm{min}$

Figure 122: Changes in CT average (2500 psig) after injecting alcohol and vacuum drying of sample.

Figure 123: Three-dimensional recreation of the fracture at 500 (left) and 2500 (right) psig.

Figure 124: Thickness aperture map of the fracture at 500 (left) and 2500 (right) psig.

\section{LIST OF TABLES}

Table 1: $\quad$ Rock properties assigned to fracture and matrix in two-dimensional model

Table 2: $\quad$ Rock properties assigned to fracture and matrix in three-dimensional model.

Table 3: $\quad$ Absolute permeabilities before and after fracturing

Table 4: $\quad$ Air permeabilities for samples cut parallel and perpendicular to bedding planes before and after fracturing.

Table 5: $\quad$ Relative permeabilities at $\mathrm{S}_{\text {wirr }}$ and $\mathrm{S}_{\text {or }}$ for fractured and nonfractured samples.

Table 6:

Water and Oil saturation at $\mathrm{S}_{\text {or }}$ condition.

Table 7:

Water and Oil saturation inside the fracture at $\mathrm{S}_{\text {wirr }}$ condition............

Table 8:

Roughness factor values

Table 9:

SMI parameters for 500 and 2500 psig.

Table 10:

Summary of the segmentation process and block dimensions.

100

Table 11:

Table 12: Liquid flow rates and fractional flow

Table 13: Fluid fracture saturation comparison at high and low pressure.........

Table 14: Volume change with confining pressure. Before and after $\mathrm{P}_{\mathrm{c}}$ changes.

Table 15:

Residual oil saturation and mobile at 500 and 2500 psig. 


\section{INTRODUCTION}

Natural and artificially-induced fractures in a reservoir have a great impact on fluid flow patterns and on the ability to recover hydrocarbons. In tight formations, the naturally fractured system provides access to the hydrocarbon fluids stored in the matrix. Fractures can have a negative effect on recovery processes when they form bypassing paths, especially in production-injection systems. For example, injected fluid may preferentially flow through the fractures leaving behind inaccessible and non-contacted hydrocarbons. Fractures can enhance the efficiency of displacement operations when the main direction of flow is perpendicular to the direction of the fractures. Fractures may also be non-conductive and form barriers to fluid flow. The mass transport between the fractures and the surrounding matrix has an important role in being able to predict and optimize the recovery processes from fractured reservoirs. As production occurs and pore pressures decline, the net confining stress on the rock increases. This project focuses on the effects that changes in confining stress have on the transport properties of the fracture-matrix system. The confining stress has an impact on the fracture aperture and therefore on multiphase fluid transport properties (Barton et al., 1985, Gentier et al., 1997). We propose to quantify the changes in fracture porosity and saturation distribution under steady and non-steady flow in both the fracture and the adjoining matrix. The quantitative changes will be obtained by low and high resolution X-ray computerized tomography (CT) imaging (Vinegar and Wellington, 1987), and then these changes will be used in an inverse simulation process to quantify changes in permeabilities and to suggest up-scaling procedures.

We propose a fracture-matrix interaction program with the following objectives:

- Quantify the effects of confining stress on fracture topology and aperture and porosity.

- Quantify the changes in two-phase fracture permeabilities as a function of stress. 
- Determine the effect of stress on oil-water transport between the fracture and the matrix.

- Determine the effects of stress on recovery processes in the presence of fractures.

- Use inverse simulation of four-dimensional saturation data for up-scaling.

We approach multi-phase flow in stressed fractured rocks experimentally and computationally. We will use multi-phase injection into confined rock samples that contain natural fractures or artificially induced ones. We will quantify the saturation distribution temporally and spatially (four-dimensional) using our new imaging facility and The Center for Quantitative Imaging. We will also monitor the pressure behavior of the sample and confining fluids. The resulting fourdimensional distribution of saturations and pressure histories will form the basis for multi-phase fluid flow simulation with the ultimate goal of quantifying the changes in the fluid flow characteristics of the rock as a function of the net confining stress. This combined experimental/computational approach will lead to advances in our understanding of the effects in-situ stress on recovery processes. 


\section{EXECUTIVE SUMMARY}

The main objectives of this project are to quantify the changes in fracture porosity and multiphase transport properties as a function of confining stress. These changes will be integrated into conceptual and numerical models that will improve our ability to predict and optimize fluid transport in fractured system. The project focuses on the four main subjects presented here:

Fracture Topology, and Aperture Distribution: Several rock samples with extensional and shear fracture were scanned the distributions of fracture apertures were obtained. There is a relationship between the structure of the fracture aperture map and the type and orientation of bedding. Some of the samples were scanned specifically for characterization purposes and some were scanned as part of fluid flow experiments. The Euler-Poincare (EPC) and the Structure Model Index (SMI) methods were used to characterize the distribution of fracture apertures and to differentiate between samples.

Matrix-Fracture interaction - Counter-Current Imbibition: Counter-current imbibition experiments between the fracture and the matrix were performed. The experiments showed that presence and the significance of this transport process, and also demonstrated the effects of different layers on the process. A balance between overall co-current flow away from the fracture as well as the counter-current flow in the fracture and next to the fracture walls was observed. The multi-phase experimental process was modeled in principal providing detailed insight into the entire process including cross layer flow, and the importance of capillary forces in the fracture.

Shear Fractures Parallel and Perpendicular to Bedding: Shear fractures have different properties than extensional fractures. We have artificially fractured several samples in shear mode and where the layers are parallel and perpendicular to the main axis of the core. The absolute permeability of the overall sample was reduced after the introduction of a shear fracture when the layers are perpendicular to the main axis of the core. The absolute permeability increased in the parallel case. The presence of the shear fracture created a significant reduction in water flooding effectiveness. This effect can be related to the morphology of the fracture and to fracture-matrix fluid interactions.

Effects of Confining Stress on Aperture Structure and Two-Phase Flow: Several experiments were carried out in which sweeps of the fractional flow domain were used to study the effect of the presence of the fracture on pressure drops (relative permeabilities) as well as the added effects of increasing the effective confining stress. Instability of the fluid distribution in the fracture let to hysteresis and sudden pressure changes during two-phase flow conditions. In some cases, the mobile saturation range in the sample was much smaller than expected or typically used in practice. This small mobile saturation range has an impact on mass transport between the matrix and the fracture in recovery processes and should be included in predictive models. 


\section{EXPERIMENTAL METHOD AND PROCEDURES}

The experimental system used in this project includes a multi-phase fluid flow system, a core holder assembly that can provide controlled confining pressure, and an x-ray computed tomography system. A schematic of the system is shown in Figure 1 and photographs of the medical and the industrial scanners are shown in our first quarterly report. Most of the details of the fluid flow system can be found in Alajmi and Grader (2000). Two new core holders were acquired. These are tri-axial cells with high pressure ratings and elevated temperatures. The rock samples may have a diameter of up to $25 \mathrm{~mm}$ and a length of up to $300 \mathrm{~mm}$. These core holders will be used to study the effects of confining stress and fracture aperture on two phase flow. A new core holder was designed and built for low confining pressure. This core holder is made of polycarbonate and is clear. The advantage of this low pressure core holder is that it has a low x-ray attenuation allowing efficient micro-tomography operations.

The Computed Tomography (CT) system consists of an ionized x-ray source, a detector, a translation system, and a computer system that controls motions and data acquisition. The x-ray source has a Tungsten target with a focal spot of 5 microns. It produces a cone beam that passes through the core and activates the detector. The image intensifier detector surface releases electrons that are then focused on a screen that is photographed by a high-resolution (1024x1024) camera with a frequency of $15 \mathrm{~Hz}$. The sample is rotated 360 degrees in the x-ray beam while the detector is providing attenuation views to the data acquisition computer. After the sample is rotated a complete turn, the system reconstructs a slice, a cross-sectional image of the attenuation values that represents a combination of the density and the apparent atomic number of the sample and the imaged position. The imager operates in volume mode where 
several separate slices are collected in one rotation. In the example shown in the report, up to 41 slices were acquired in a single rotation. After each rotation, the sample is translated axially to a new scanning position, thus, allowing a continuous three-dimensional coverage of the sample. 


\section{RESULTS AND DISCUSSION}

\section{Fracture Topology, and Aperture Distribution}

Methods: Most of the cores used in this research were $25 \mathrm{~mm}$ in diameter. Thus, with the use of the high-resolution scanner we were able to obtain voxel size on the order of 30 microns, inside a core holder or un-confined. Hence the overall best resolution of fracture apertures that we can obtain was about 30 microns. We note here that the virtual digital fracture is composed of parallelograms called voxels, and that all the characterization of the fracture volume is done in this digital world. All the samples in this work were artificially fractured by either a modified Brazilian test or by shear fracturing in a Hoek cell (as described in early reports). There are two methods for scanning extensional fractures. The first method is to scan the sample with the fracture completely gaped (no asperities). This method produces two surfaces that can then be studied, characterized, and used digitally to create different asperity sets by collision and orientation methods. The second method is to match the two parts of the core - with a possible translation to create focal asperities in some cases - and scan the core with the fracture. The work presented here uses the second method. In the case of shear fractures, the samples were sealed prior to fracturing and were never opened. Thus, the gouge and fractured zone were preserved and scanned with loss of mass.

Each sample was scanned under dry conditions (in fluid flow cases, under vacuum), and the high-resolution virtual samples was created from the CT data. Figure 1 shows an example of a single image with a highlighted section to its right. The image shows the vertical layers and the expanded region shows large pores (black spots), high density elements (white spots) and the fracture. Through numerical processes that include erosion and dilation, a two-dimensional map 
of the distribution of fracture aperture was extracted from the entire data set, shown in Figure 2. The red areas are asperity regions where the two sides of the fracture are in contact. The white regions are regions of high fracture width of about 1200 microns. The yellow contour represents a fracture width of 600 microns. This type of analysis permits the computation of fracture volume, as well as to demonstrate the topology of the fracture. Dark regions would be filled with water, and light colored regions will form oil flow channels. We expect to perform fluid flow experiments in similar fractured samples. The pre-determination of the fractured region will simplify the partitioning of the two phases in the fracture during the post-acquisition analysis stage.

Extensional Fractures: The method of creating the extensional fractures is presented in the first Quarterly Report. After the original CT volume was reduced to contain the fracture the fracture was characterized by three-dimensional visualization. Fracture aperture and volume were computed. This process was applied to different confining pressure. Figure 3 shows the core fracture (in red) surrounded by the matrix. Figure 4 presents an iso-surface of the fracture at 500-psig confining pressure (left). In order to differentiate the fracture from the matrix a threshold must be applied. Further verification of the used threshold will be studied in the future. Figure 4 shows many asperities (dark color) in the fracture, these asperities are fracture contact points and are responsible for the reduction of the conductivity the fracture. 


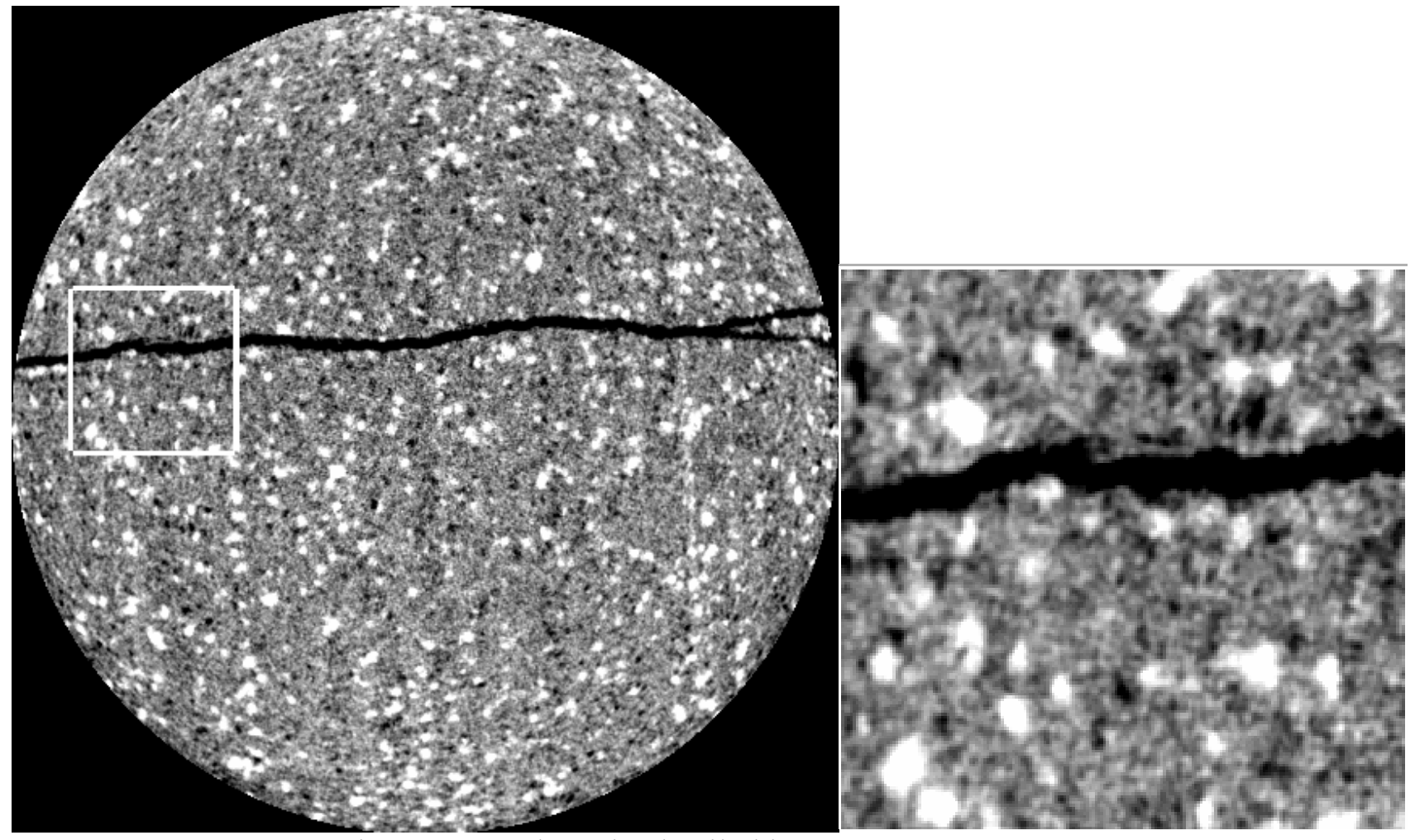

Figure 1: Fractured Berea sample and a detailed inset.

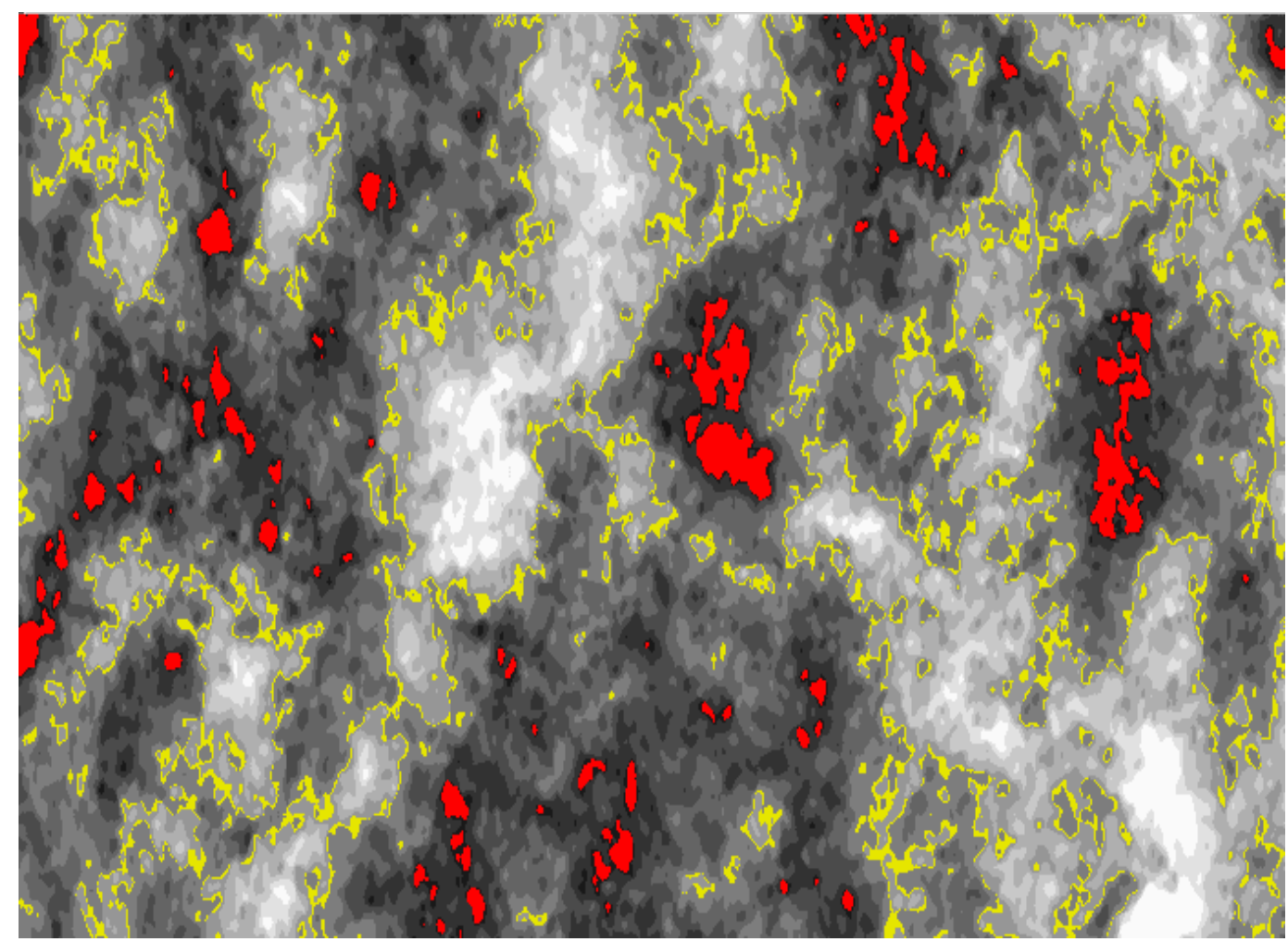

Figure 2: A two-dimensional map of fracture aperture. Area: $16 \mathrm{~mm}$ x $30 \mathrm{~mm}$.

Red: Contact regions. White: aperture of 1200 microns. Yellow: 600 microns contour. 

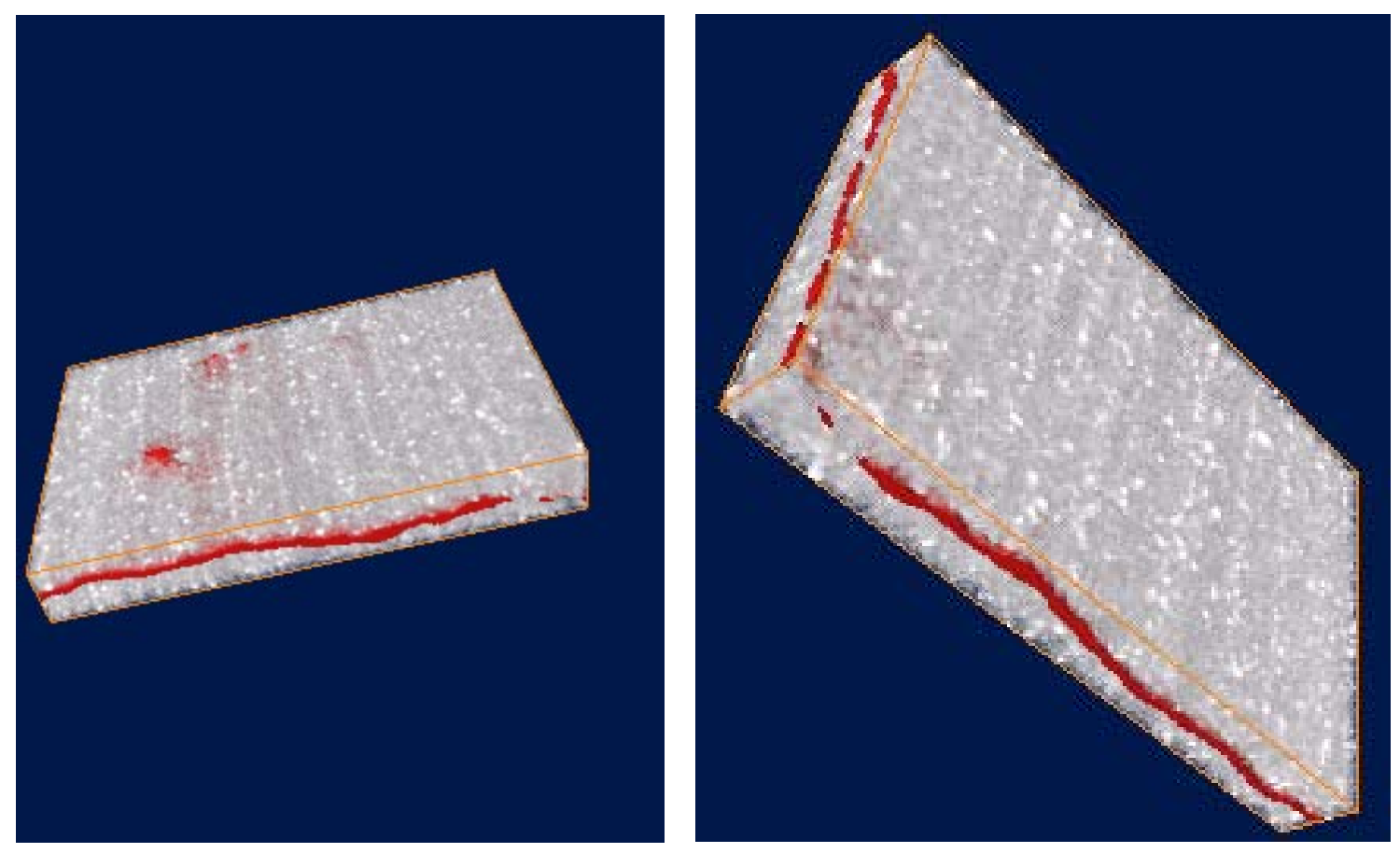

Figure 3: Fracture surrounded by matrix.
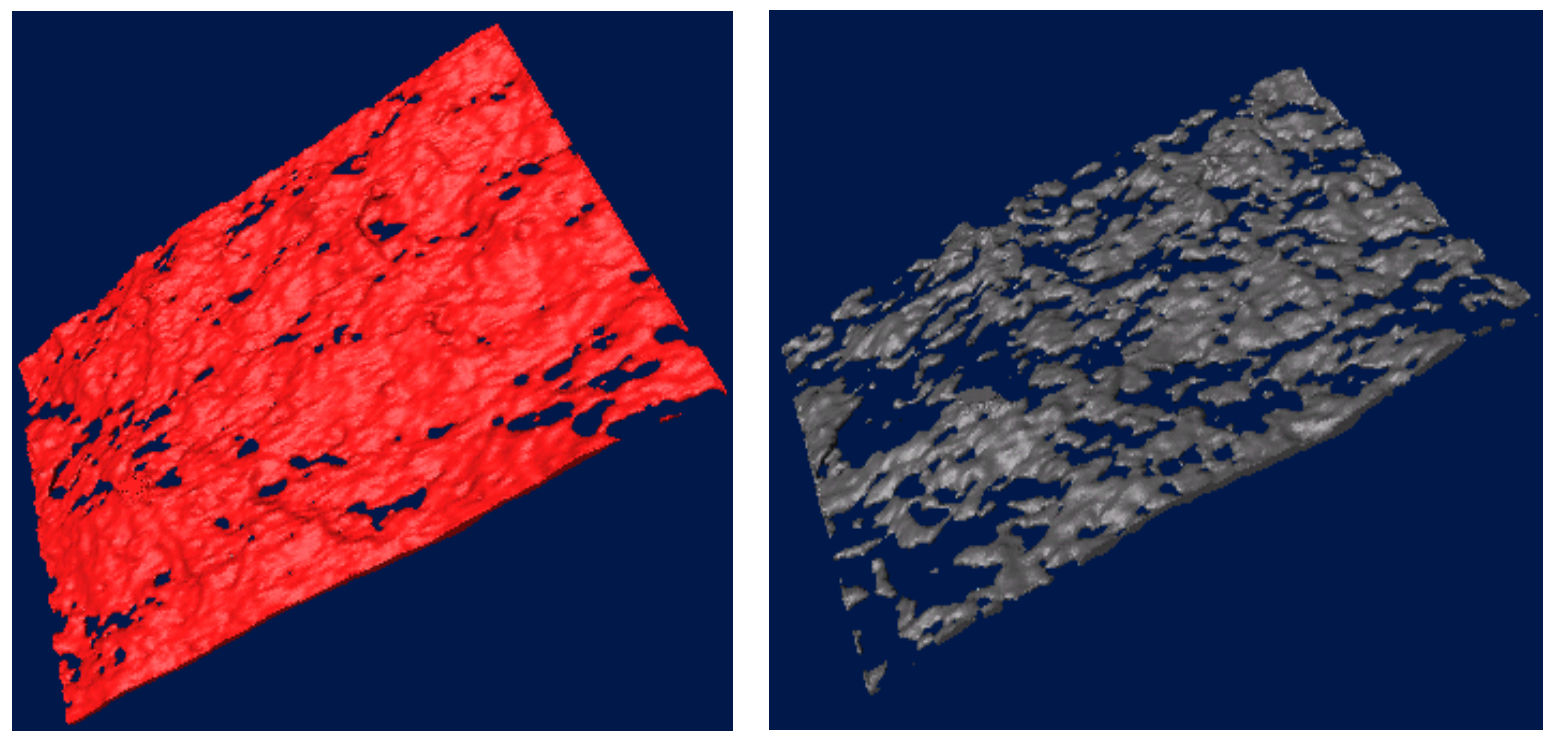

Figure 4: Three-dimensional images of the fracture under 500 and 2500 psig confining pressure. 
Figure 4 (right) shows the fracture under a much higher confining pressure, 2500 psig. There is an increase in the size of the asperities (dark color) as a consequence of the increment in the confining pressure.

Fracture Aperture vs. Porosity in Neighboring Layered Rock: In the past, a correlation between the fracture aperture and the porosity of adjacent layers has been observed. The evidence was mainly hydraulic. Micro CT provides an opportunity to explore this correlation from mechanical and topological points of view. Fracture aperture and porosity distribution in the neighboring rock matrix were extracted from X-ray CT images of a layered Berea sandstone sample. This sample with a diameter of $51 \mathrm{~mm}$ was fractured perpendicular to rock layering, as shown in Figure 5.

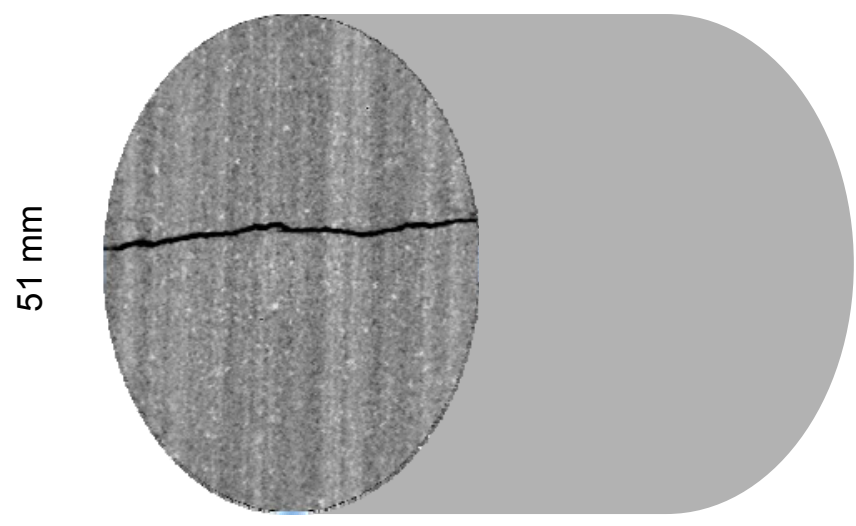

Figure 5: Berea sandstone sample with fracture perpendicular to rock layering.

A total of 300 images were taken along the main axis of the cylindrical sample, for an actual scanned section of $10 \mathrm{~mm}$. A smaller section of the sample is extracted for further mathematical manipulation, shown in Figure 6. This section contains the fracture void and a portion of the surrounding rock structure. Through a three-dimensional connectivity search, all interconnected elements in the fracture void space are identified and extracted. The CT signature of all pixels in 
the fracture is enhanced to define a clear fracture-matrix interface. The two images in Figure 6 contain 983 pixels in the horizontal direction and 261 pixels in the vertical direction. Figure 6a shows the original MicroCT image and Figure $6 \mathbf{b}$ shows the same image with the fracture volume highlighted after the connectivity search.
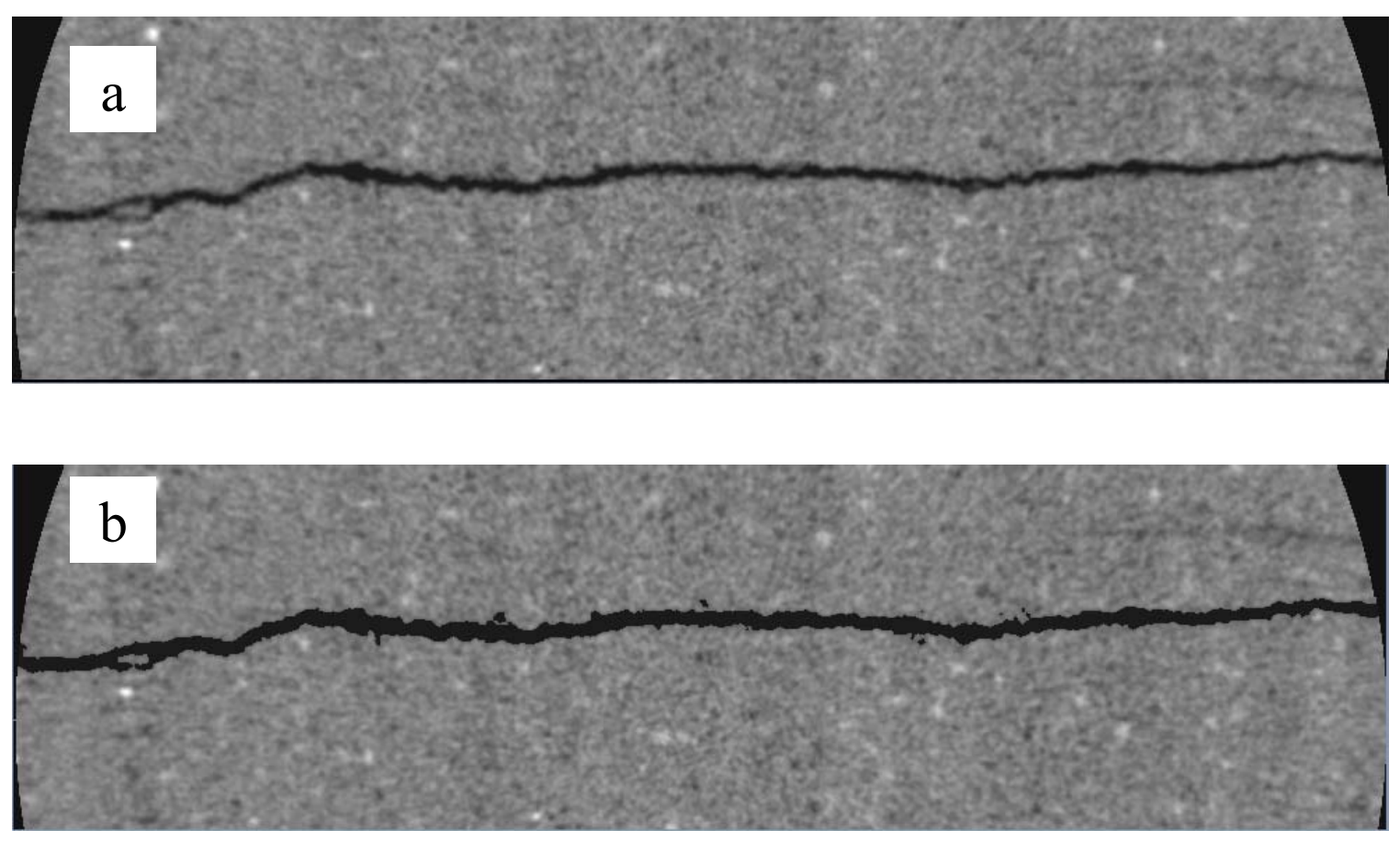

Figure 6: Examples of original CT data (a) and connected fracture volume (b). Image size had been cropped to $983 \times 261$ elements to reduce the computation effort.

The relationship between fracture aperture and adjoining porosity was quantified by comparing vertical fracture apertures to matrix porosity at various locations and block sizes. Figure 7a shows a portion of the fracture-matrix system with the blocks divided into four groups: black represents the fracture, white represents the matrix, red represents fracture aperture at a given location, and blue represents the adjoining matrix blocks that were used to determine the porosity for the specific fracture aperture. Figure $7 \mathbf{b}$ shows an example of large blocks (in the $\mathrm{x}-\mathrm{z}$ direction highlighted in green) that were used to determine a correlation between aperture and porosity. 

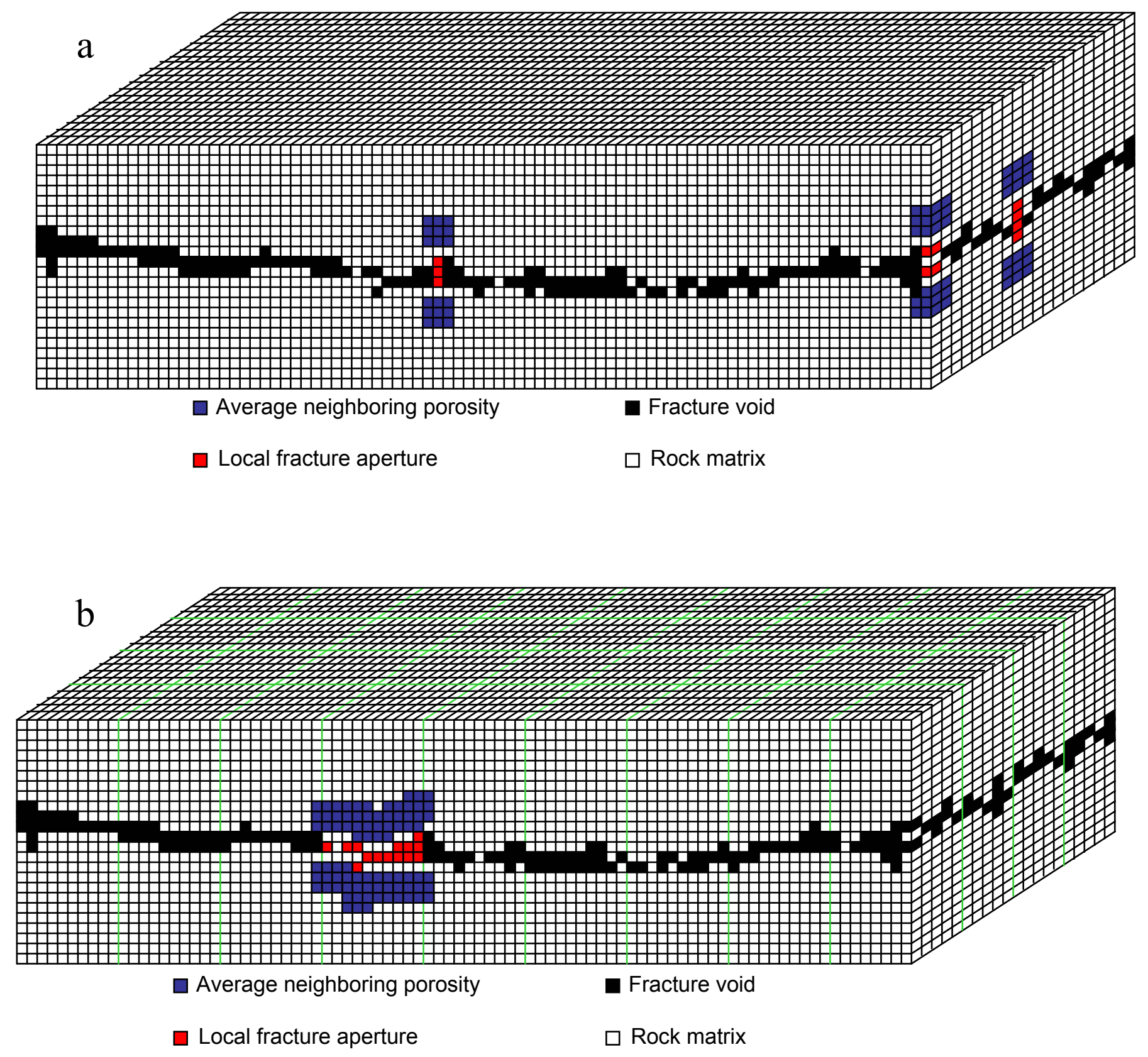

Figure 7: Examples of fracture and matrix regions used in correlating aperture with matrix porosity. 
Different section sizes were tested and neighboring CT porosity and local apertures were correlated in each case. Porosities and apertures were computed for data blocks (sections) of 10pixels by 10pixels (x-direction by z-direction), 15 x 15, 20 x 20, 30 x 30, 40 x 40, and 50 x 50 . In each case, CT porosity and fracture aperture were linearly correlated. The lowest dispersion of points was obtained for blocks of 15 x 15 pixels, shown in Figure 8.

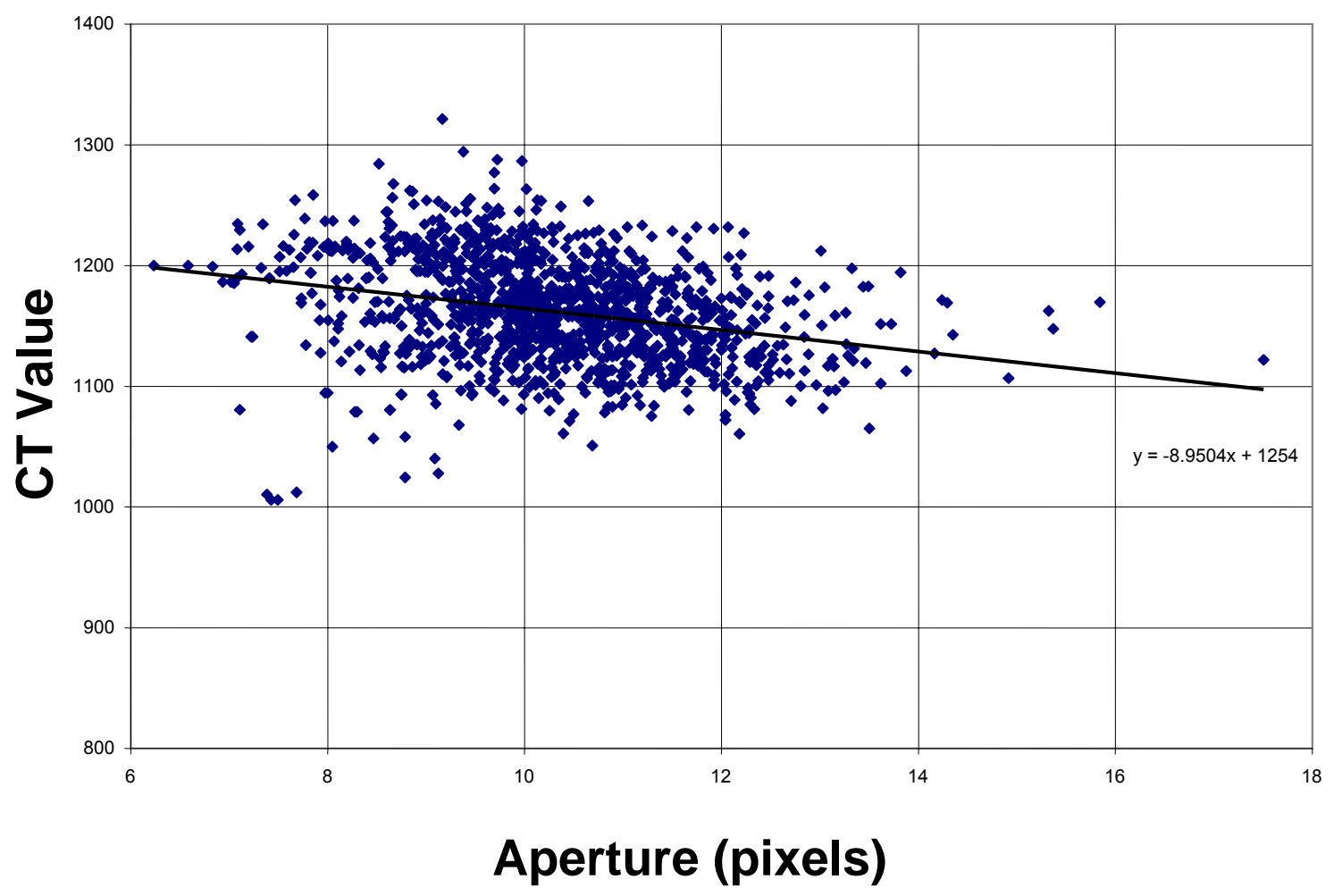

Figure 8: CT value as related to porosity as a function of fracture aperture.

A Linear Least Squares fit for the data shows a correlation with a negative slope. This negative slope implies that fracture aperture and CT values are inversely proportional, the higher the aperture the lower the $\mathrm{CT}$ registration. A low $\mathrm{CT}$ registration is characteristic of media with low density or high porosity Regions with higher neighboring rock porosities will lead to larger apertures than those regions with lower neighboring rock porosities. The reasons for the 
relationship between fracture aperture and the properties of the adjacent rock are not fully understood. Some explanation for this observation rests in the method of fracturing and fracture propagation, fracture zone, rock structure and cementation.

Two-dimensional fracture aperture and neighboring porosity maps were generated to obtain a visual representation of the relationship between fracture aperture and the properties of the adjacent rock. The arithmetic average of the $\mathrm{CT}$ registrations in the neighboring rock is calculated for each point on the fracture plane. Colors blue and red in Figure 9, represent the topography of calculated adjacent porosity and fracture apertures, respectively. Figure 10 shows two axial images showing fracture aperture (a) and porosity above and below the fracture (b).

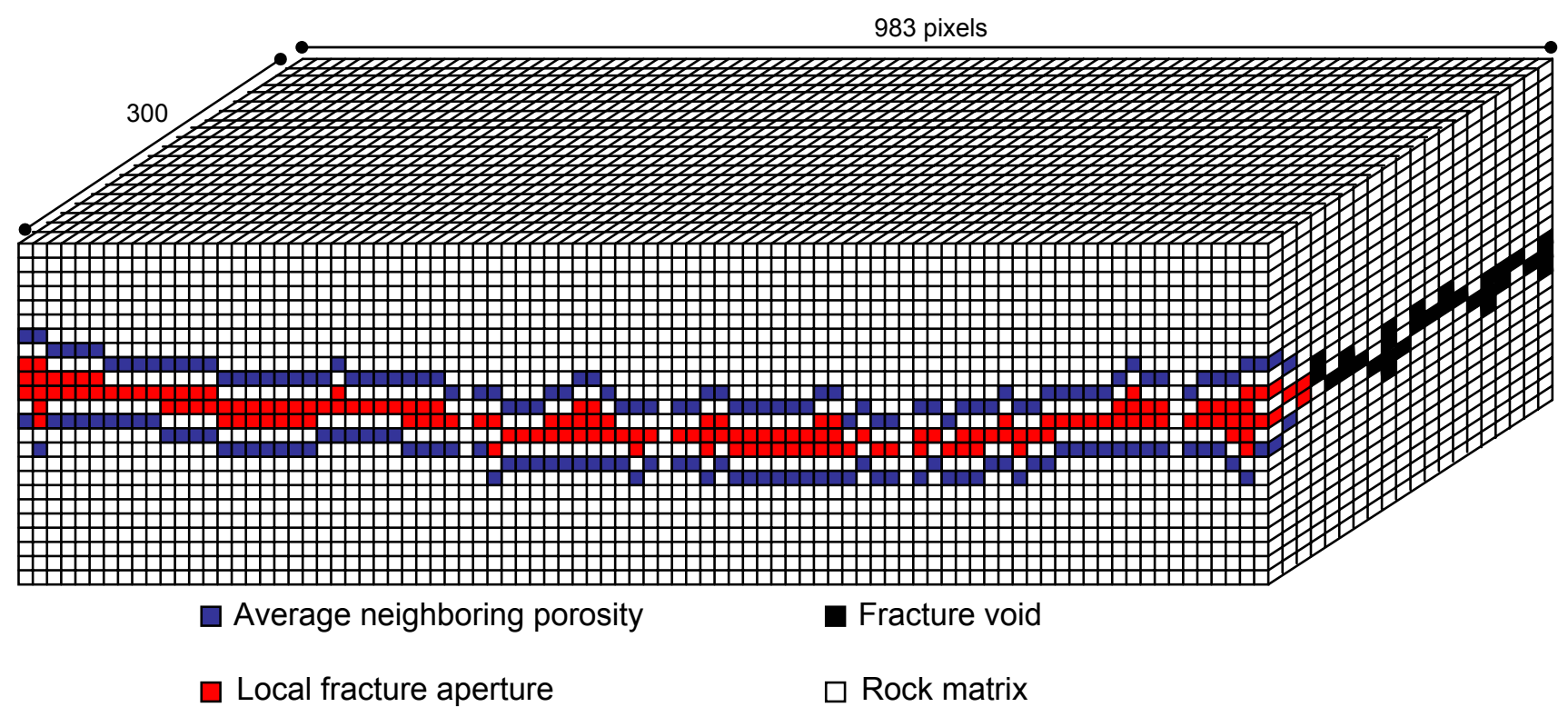

Figure 9: A representation of a portion of the fracture and adjacent matrix showing the matrix portion used in computing the adjacent porosity to the fracture. 


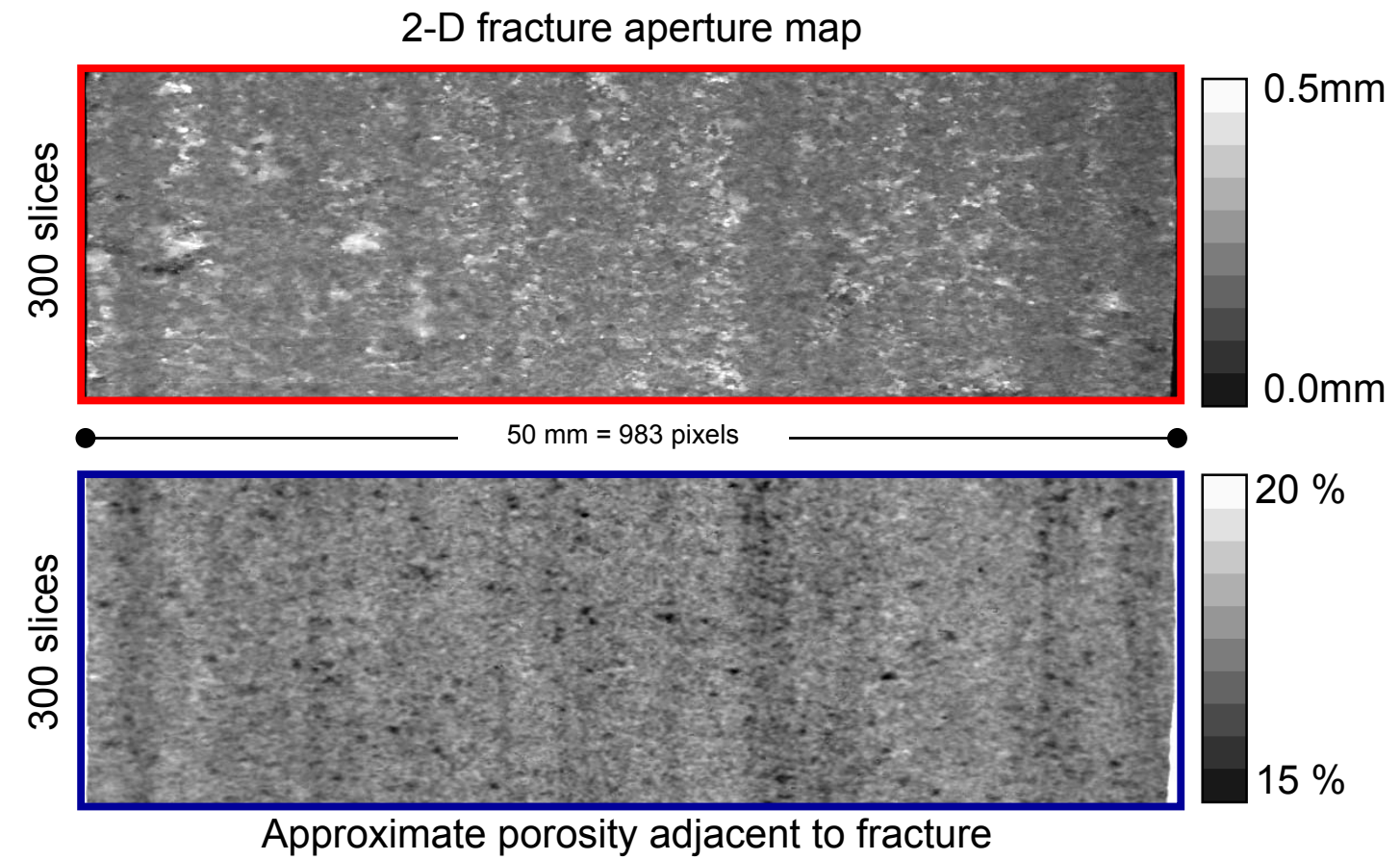

Figure 10: Fracture aperture and adjacent porosity axial images.

Three single slices were also selected out of the three hundred that form the sample: slice number 20 (green), slice number 140 (orange), and slice number 280 (red) shown in Figure 11. The relationship between fracture aperture and adjacent porosity was also evaluated in these slices and compared to the general trend. Layering can be visually identified in both aperture and porosity maps. Strong correspondence can be observed when comparing layering in both twodimensional maps. In addition, different layers will show different level of correlation. The correspondence between aperture distribution and rock layering is highlighted with vertical black lines in Figure 11. 


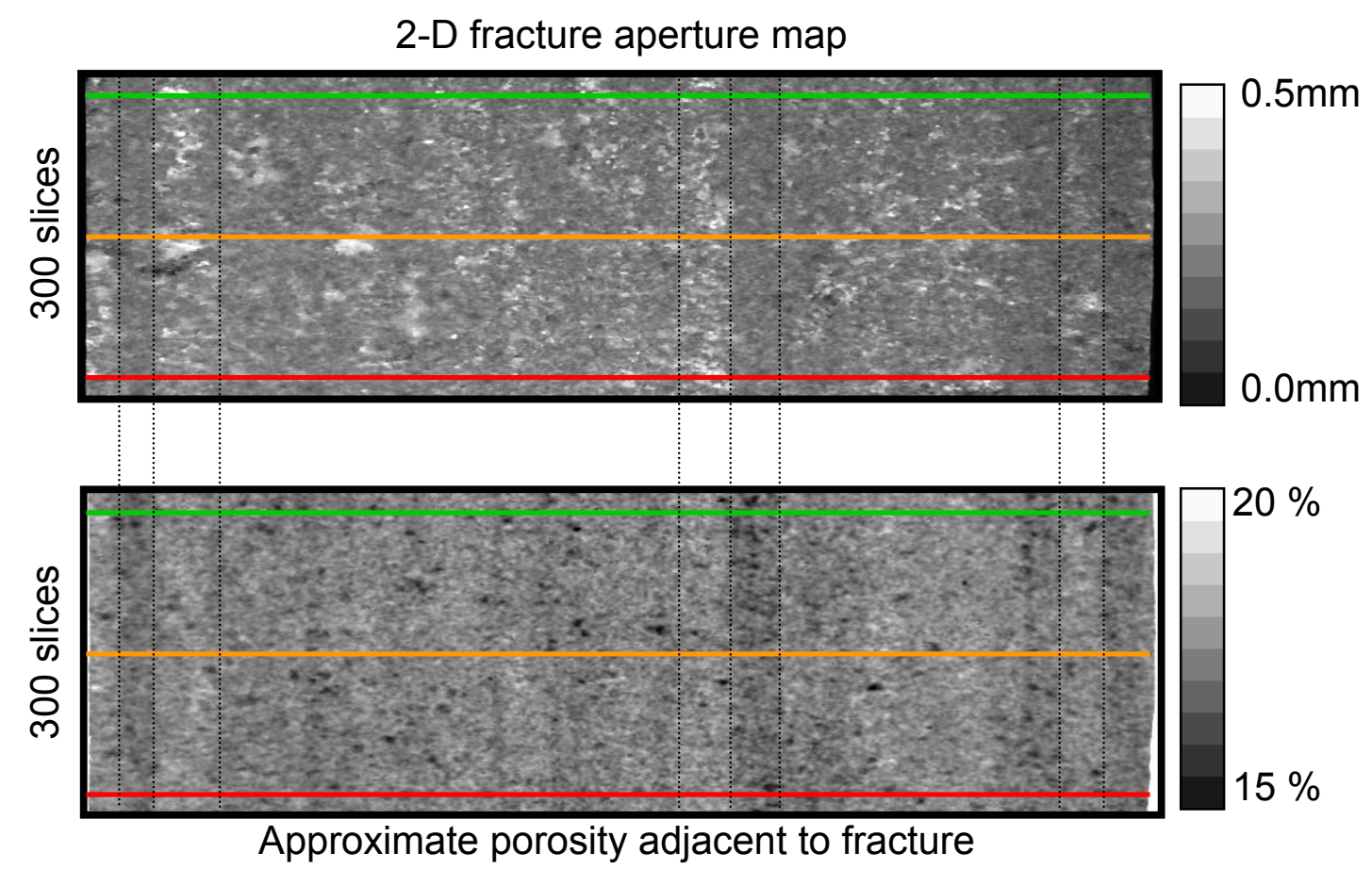

Figure 11: Fracture aperture and adjacent porosity axial images showing the specific slices (20 - green, 140 - orange, 280 - red) and layers that are strongly correlated (black vertical lines).

Shear Fractures: The method for creating the shear fractures is detailed in the sixth semi-annual report. Again, two types of cores were used: with the layers parallel to the main axis of the long core, and with the layers perpendicular to the long axis of the core.

A software package named Amira was used to visualize three-dimensional data sets. A state-ofthe-art visualization and quantification technique helps to gain detailed insight into the data. The images were used to extract three-dimensional rendering and surface generation of the fracture itself from the rock matrix.

It was impossible to extract the fracture globally using a threshold CT value due to the significant differences in the CT values of the fracture in the high density and low density layers. The CT numbers of the fracture zone in the high density layers matched that of the CT numbers 
of the matrix in the low density layers. The damaged zone or the gouge formation formed near the fracture also contributed to the difficulty in identifying the fracture. Therefore, for a given slice, the fracture was extracted manually by a combination of setting thresholds and testing connectivity of pixels. Extracted fracture data were used to create three-dimensional visuals of the fracture.

Figure 12 shows an a three-dimensional image of the fracture consisting of all voxels less than set threshold is highlighted in red and the layers of the sample are shown in a grayscale where high density material is in light gray. The fracture geometry is complex and does not lie along a straight plane from one end to the other. Much of this complexity is likely due to end effects where loading pistons contact the sample. The high density layers cutting across the fracture, which are light in shade and are perpendicular to the fracture, are also clearly visible in the figure.

The connected pores in the fracture can be seen in Figure 13. Here, only fracture voxels actually connected to each other are shown. The increase in the permeability after the fracturing can be attributed to the fact that the majority of the fracture voids are connected. The fracture was further characterized by identifying the major connected pores and calculating the volume of the fracture. Volume of the fracture was $75 \mathrm{~mm}^{3}$, which is about $0.2 \%$ of the total sample volume. Figure 14 shows another view of the fracture surface head-on, highlighting the large asperities. 


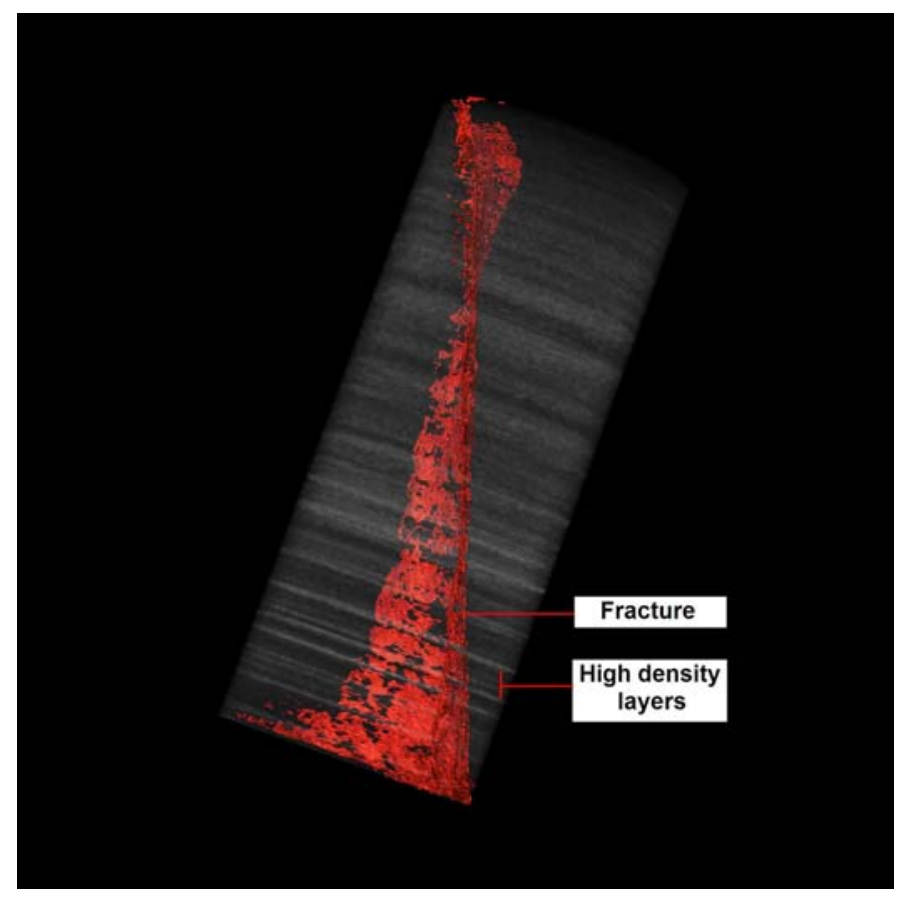

Figure 12: A three-dimensional rendition of the fracture.

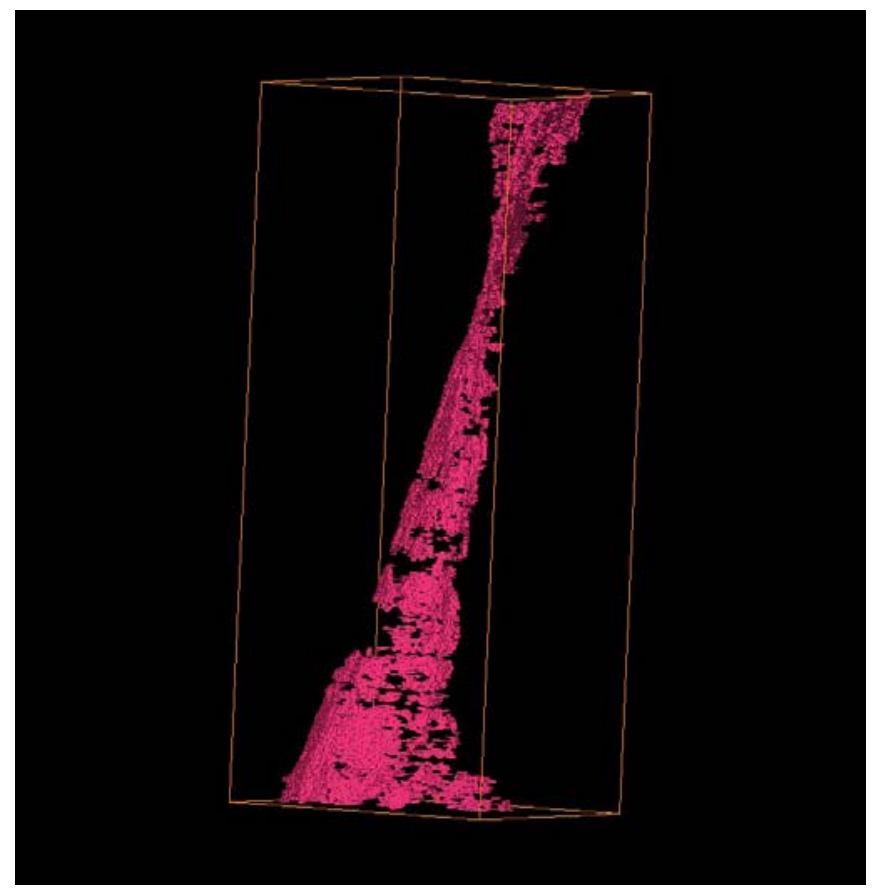

Figure 13: A three-dimensional rendition of the connected volume of the fracture. 


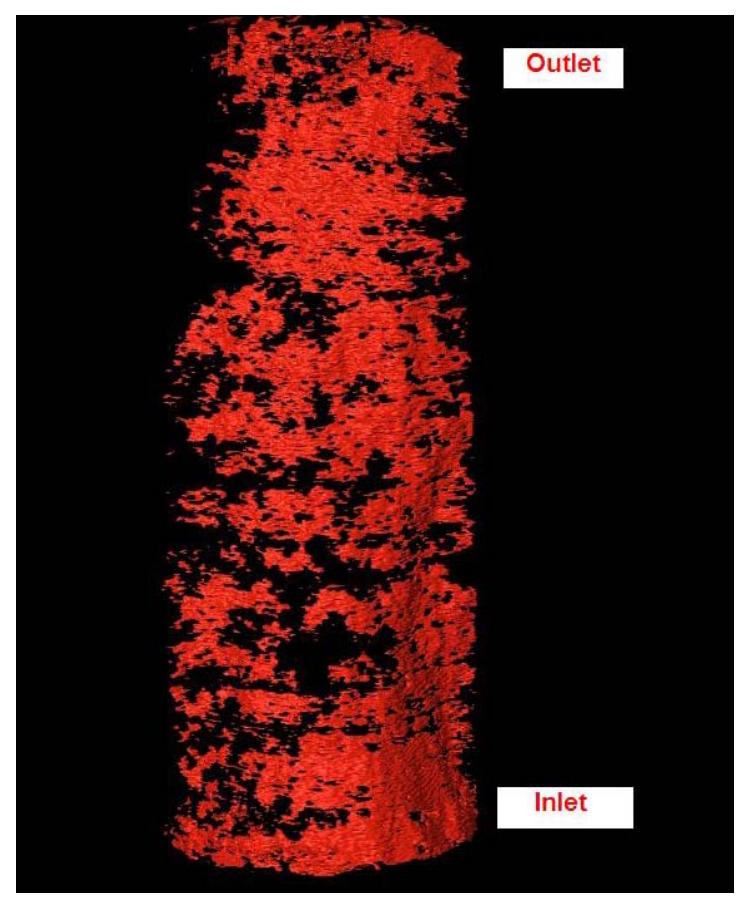

Figure 14: A three-dimensional rendition of the fracture. Black spaces indicate asperities.

The effects of confining pressure on fracture morphology and the use of the Euler-Poincare (EPC) and the Structure Model Index (SMI) are presented in a later section.

Matrix-Fracture Interaction - Counter-Current Imbibition: Much of the research in this area was presented in the sixth semi-annual report. The rock sample used in this experiment (Sample 2) was cored parallel to bedding and fractured perpendicular to bedding. Once the rock sample is fractured and separated into two halves, the two portions of rock are put back together with a slight shift of $0.4 \mathrm{~mm}$ along the longitudinal axis. This shift accentuates aperture changes inside the fracture. 0.4-thick plates were placed at the bottom of the core to maintain a flat contact with flow distributors. The pixel resolution used in this experiment was $0.030 \mathrm{~mm} \mathrm{x}$ $0.030 \mathrm{~mm}$ and the slice thickness was $0.032 \mathrm{~mm}$. Detail procedure of experiment 2 is described in the sixth report. A schematic representation of the scanning sequence is shown in Figure 15. 


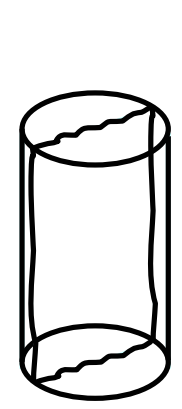

Dry
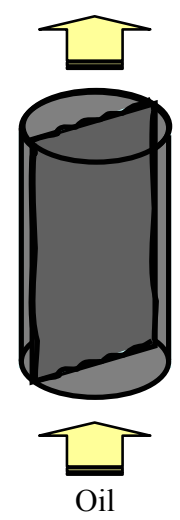

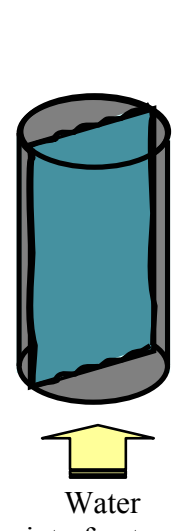

into fracture
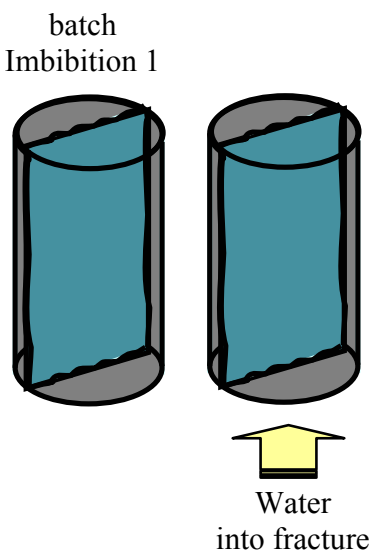
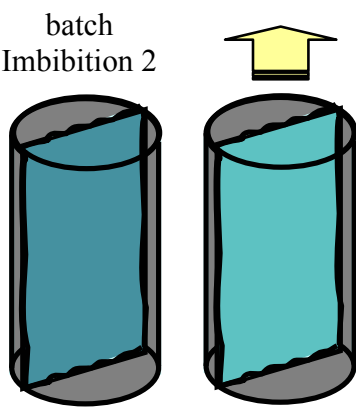

continuous

imbibition

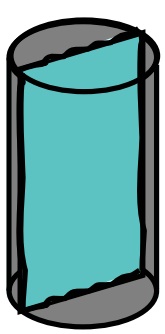

Figure 15: Scanning sequence of experiment 2: capillary imbibition.

Data collected during spontaneous imbibition include high-resolution scans at a fixed location and large-range digital radiographs. Fluid exchange takes place as represented in Figure 16.

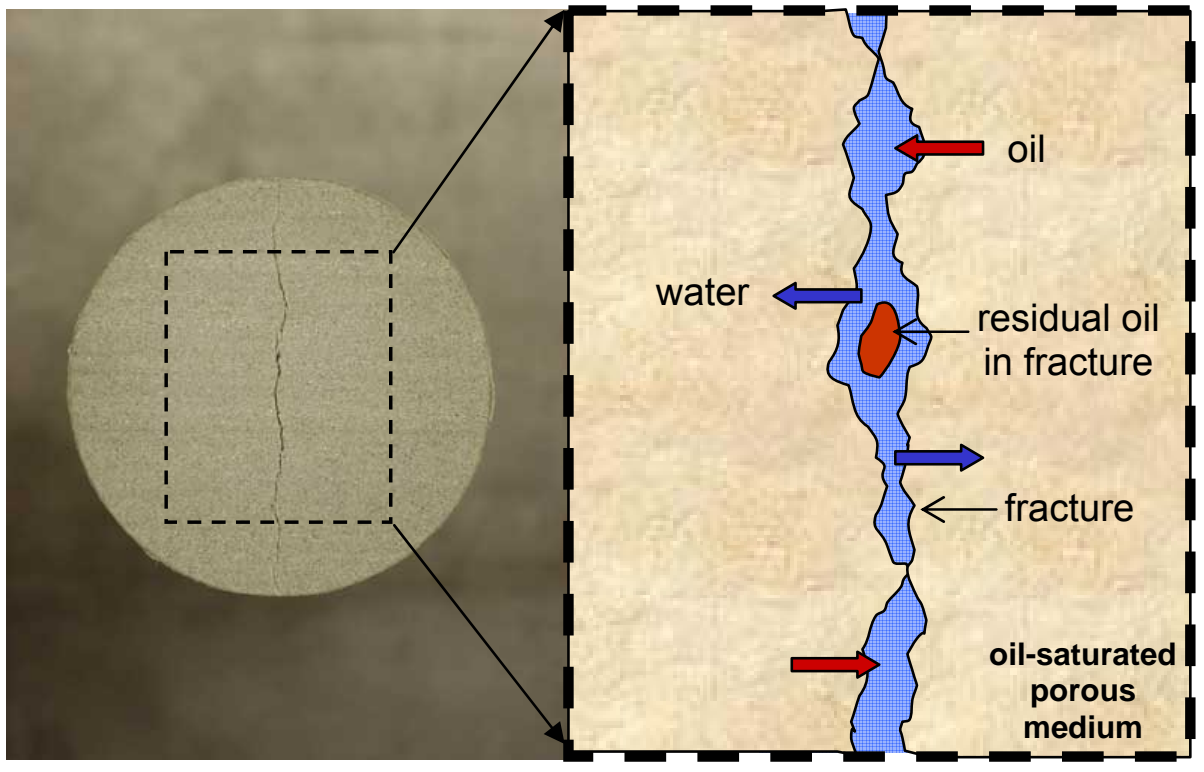

Figure 16: Schematic representation of capillary imbibition across fracture-matrix interface. 
Capillary Imbibition across Fracture-Matrix Interface: In reservoirs with water drive, either from an aquifer or from water-flooding, the oil in the matrix adjoining a fracture is generally considered to be produced by water imbibition (Lee and Kang, 1999). In an attempt to study this mechanism, an imbibition experiment was conducted, allowing a wetting phase to enter the fracture and imbibe into the adjacent matrix. In addition to the experimental work, a series of simulation scenarios were performed to assist in understanding observations and the flow mechanisms taking place during capillary imbibition in fractured media. The experiment was performed in three stages: first injecting two small amount of water, and finally injecting water continuously. The sample used was a cylindrical Berea core, $47.5 \mathrm{~mm}$ long and $25.4 \mathrm{~mm}$ in diameter with a single longitudinal fracture with open ends.

The first stage of the experiment, stage 1 , consisted of monitoring imbibition of wetting phase (water) in a fractured rock originally saturated with non-wetting phase (Soi=1.0). The rock had a single fracture in it, which extended along the full sample height. Water was injected upwards into the fracture for four minutes at a rate of $0.05 \mathrm{cc} / \mathrm{min}$, for a total volume injected approximately equal to the fracture void volume. The system was then closed and monitored using fast digital radiography (DR) and high-resolution CT scans. After several hours, when the imbibition process became too slow for changes to be appreciable, a second water injection took place (stage 2) and the system was again closed and monitored. In this second stage, a wetting phase imbibed into a sample containing both wetting and non-wetting phase $(\mathrm{Soi}<1.0)$. The third stage consisted of monitoring imbibition during continuous water injection. Fracture inlet and outlet were always open to flow during this stage. In the present document, only results from capillary imbibition 1 are described and analyzed. 
Digital Radiography (DR) is an X-ray imaging technique with rapid digital processing that allows a much faster image collection than high-resolution CT scans do. However, speed is counterbalanced by limited image quality. DR was used in this experimental work to monitor the entire core during early-time saturation changes, which could not be accomplish using high resolution images. DR and $\mathrm{CT}$ scans also have a fundamental difference in the way images are collected. CT scans are used to visualize single slices through the object. A pile or stack of CT scans adjacent one another can therefore reproduce a three-dimensional digital view of the scanned object. DR reproduces not a slice but a full two-dimensional projection of the object exposed to X-rays. From DR images it is not possible to render a three-dimensional view of the scanned object. Figure 17 presents a DR of sample 2 at initial conditions, fully saturated with the non-wetting phase. The color scheme in this figure represents the overlapped effect of the object's density and thickness. The vertical fracture is displayed in dark gray, meaning a reduced attenuation of X-rays penetrating the sample. Similarly, gray tones go from light to dark from the center outwards as the resistance is less due to the cylindrical shape of the core sample.

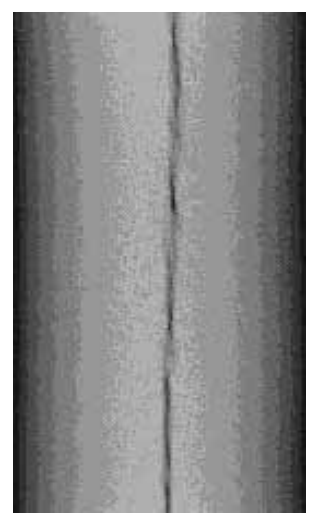

Figure 17: Digital Radiography of sample 2 initially saturated with oil. 
To enhance the definition of the water front during matrix imbibition, the initial oil saturated DR image was subtracted from the DR images collected during the imbibition period. The outcome of this subtraction, presented in Figure 18, is a series of cumulative water saturation maps during capillary imbibition where water saturation increases with brightness. The nature of these images is merely qualitative; no saturation values have been assigned the DR's since they represent a lumped registration of a three-dimensional image.

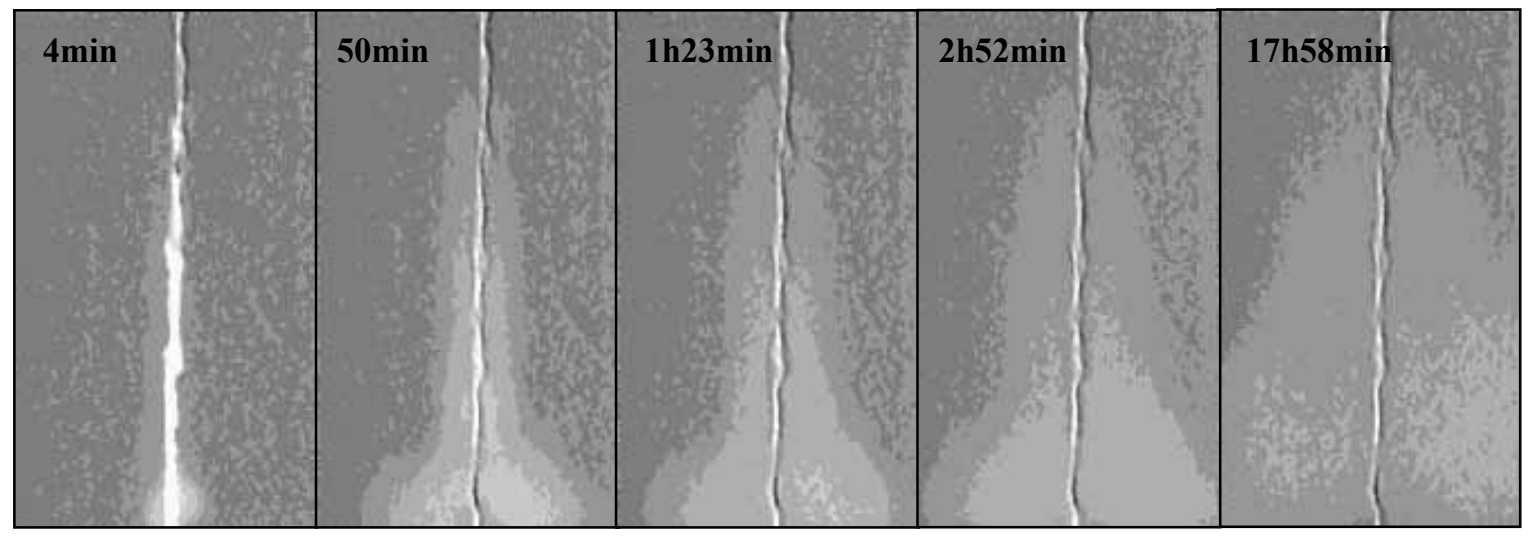

Figure 18: Cumulative water saturation during capillary imbibition obtained from digital radiography.

After four minutes of water injection $(0.05 \mathrm{cc} / \mathrm{min})$ the system was closed and water continued to imbibe into the matrix under counter-current flow; oil is simultaneously migrating in the opposite direction (towards the fracture). When injection ceased, the fracture and its immediate adjacent matrix undergoes drainage.

The inlet face of the core was covered with Teflon, leaving a narrow gap for fluid to enter directly into the fracture. Nevertheless, it was quite difficult to fully isolate the face of the core 
from the injection channels in the flow distributor so that there was still some contact between the rock matrix and water in the inlet flow distributor. That is the main reason for a significantly wider saturation front in the lower portion of the DR (Figure 18). The bell-shape saturation front is especially visible at 50 minutes of monitoring time. The extent of water penetration into the matrix from the fracture is influenced by gravitational effects holding water in the lower portion of the core and also by the amount of water being exposed to the fracture-matrix interface at a given time. During upward injection, the lower section of the core is exposed to more water than the upper section at any given time. If matrix capillary forces are high enough to pull water from the fracture before it reaches the top, then a wider water saturation front develops at the bottom of the core.

Images presented in Figure 19 were also obtained from the capillary imbibition experiment. These images represent the incremental changes in the water saturation profile between successive scans. The first image, e.g., is the result of the 0 -minute image subtracted from one obtained after 4 minutes, the second image is the result of the 4-minute image subtracted from one obtained after 50 minutes, for a total of 46 minutes, etc.

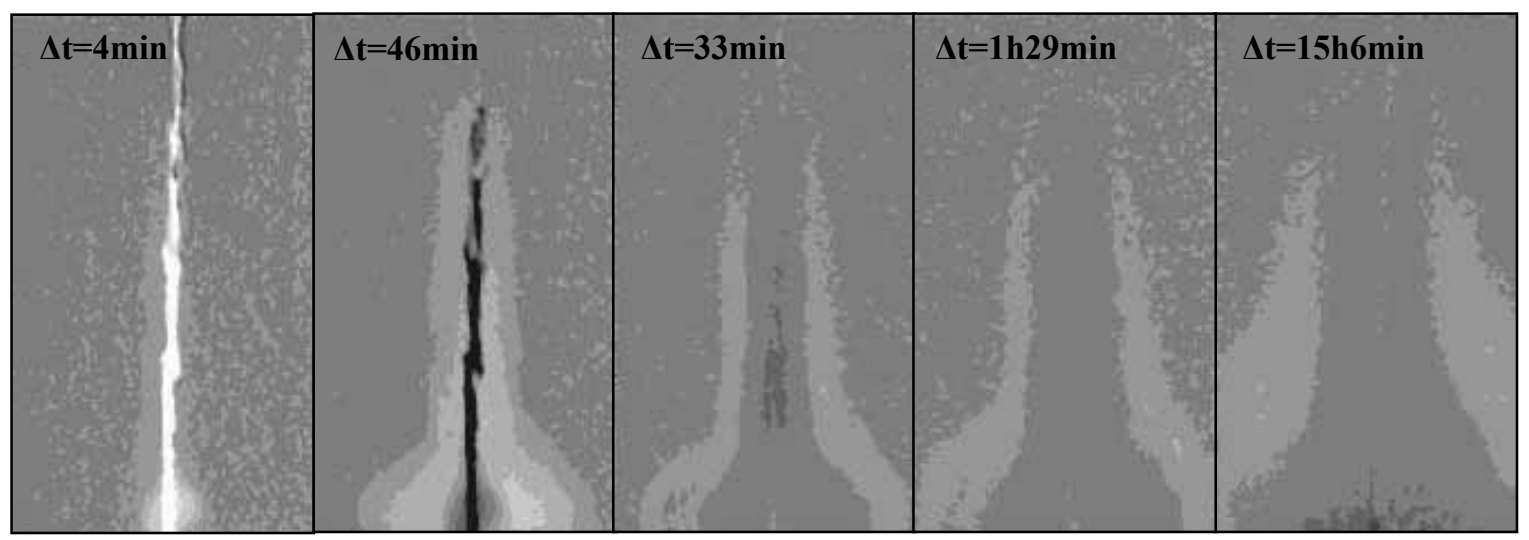

Figure 19: Incremental water saturation during capillary imbibition obtained from digital radiography. 
The change in water saturation increases with brightness. Light gray represents regions where water has arrived (imbibition), dark gray represents regions from which water has been pulled (drainage), and middle gray regions experienced little change in saturation for the labeled time period. For instance, the 4-minute time frame image shows a very bright fracture indicating the arrival of water filling this space. In the next image $(\Delta \mathrm{t}=46 \mathrm{~min})$, water saturation decreased in the fracture, while increasing in the adjacent rock. Water invasion grows not only in the horizontal direction, but there also exists a vertical component that pulls the water upwards. The last frame in Figure 19 shows a significant advance in the vertical direction with water flowing from the center bottom of the core.

Image subtraction was also applied to high-resolution CT scans taken $3 \mathrm{~mm}$ above the bottom of the core. Results are shown in Figures 20 and Figure 21. 

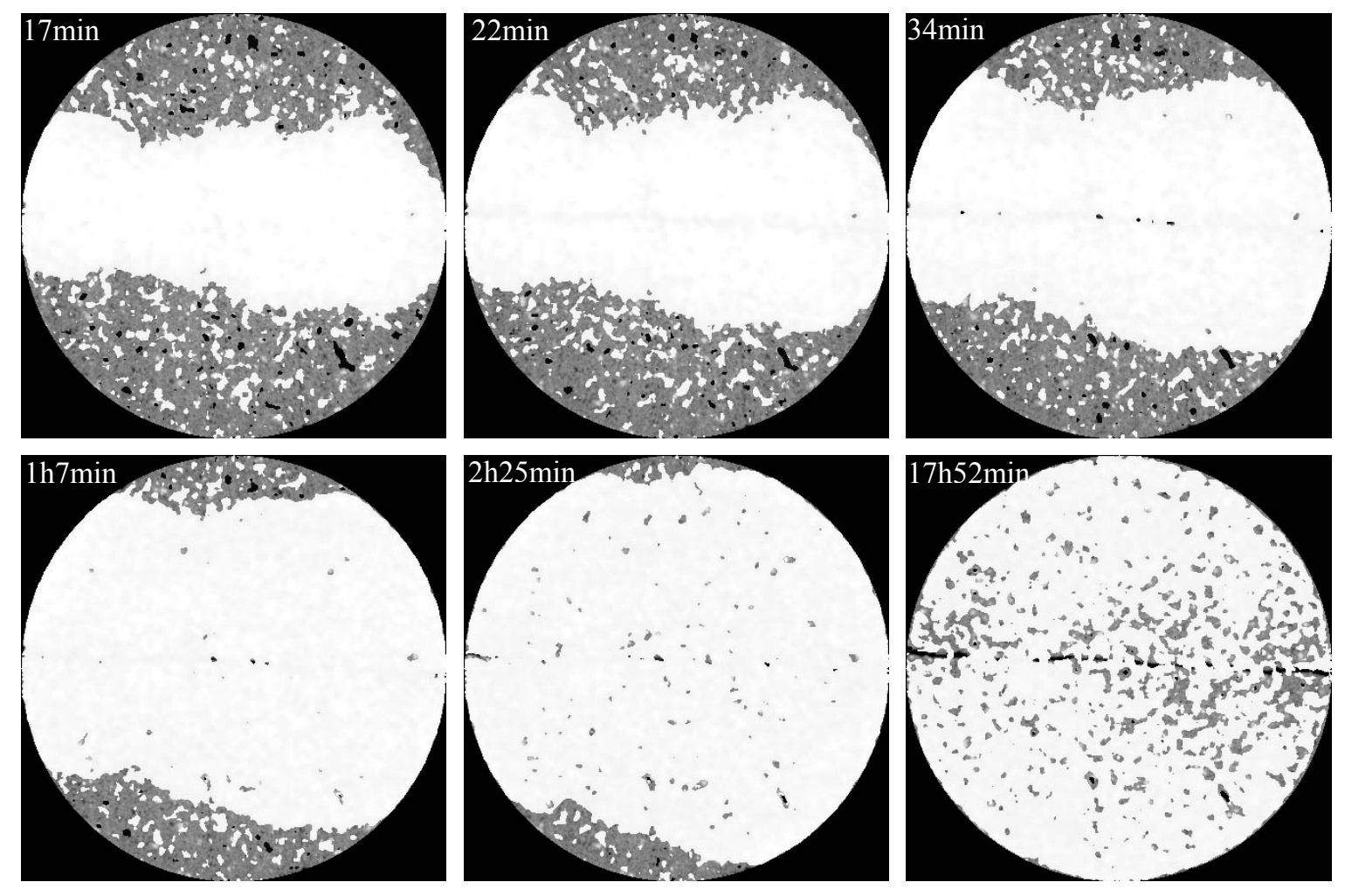

Figure 20: Cumulative water saturation during capillary imbibition obtained from highresolution CT scans.

Figure 6.20 presents a series of cumulative water saturation maps where water invaded zones are denoted in white without consideration of saturation. The remaining areas in gray represent the original rock structure without water. Cumulative CT scans in Figure 6.20 show an uneven growth of the invading water front, which is mainly due to rock heterogeneities and boundary effects. The rock sample used in this experiment (Sample 2) was cored parallel to bedding and fractured perpendicular to bedding. Thus the wavy water front is mostly caused by the contrast between the different layers in the matrix. The last image, corresponding to 17 hours and 52 minutes of spontaneous imbibition, shows sparse spots with a reduction in water saturation with respect to the previous image at 2 hours and 25 minutes. These spots are shown in gray and black, and remained after the complete water invasion, suggesting that water is transported in the 
direction perpendicular to the CT scanning plane. Recall that DR's also showed a significant water advance in the vertical direction, perpendicular to the CT scanning plane, with water flowing from the center bottom of the core.

Figure 21 shows the incremental changes in water saturation during spontaneous imbibition. White represents imbibed regions, black represents drained regions, and the remaining gray areas show the original rock structure where no significant change in saturation has taken place for a given time period. The first image in this sequence, at 17 minutes, is the same for both cumulative and incremental saturation distributions. The effect of rock layering on the development of the imbibing front is dominant during early time imbibition. Black spikes perpendicular to the fracture clearly follow rock layering patterns in the 5-minute frame in Figure 21. These spikes tend to correspond to layers with low density, which are characteristic of high porosity/permeability. The presence of these black spikes indicates that drainage is preferential to high porosity/permeability layers after they were initially invaded by water. 

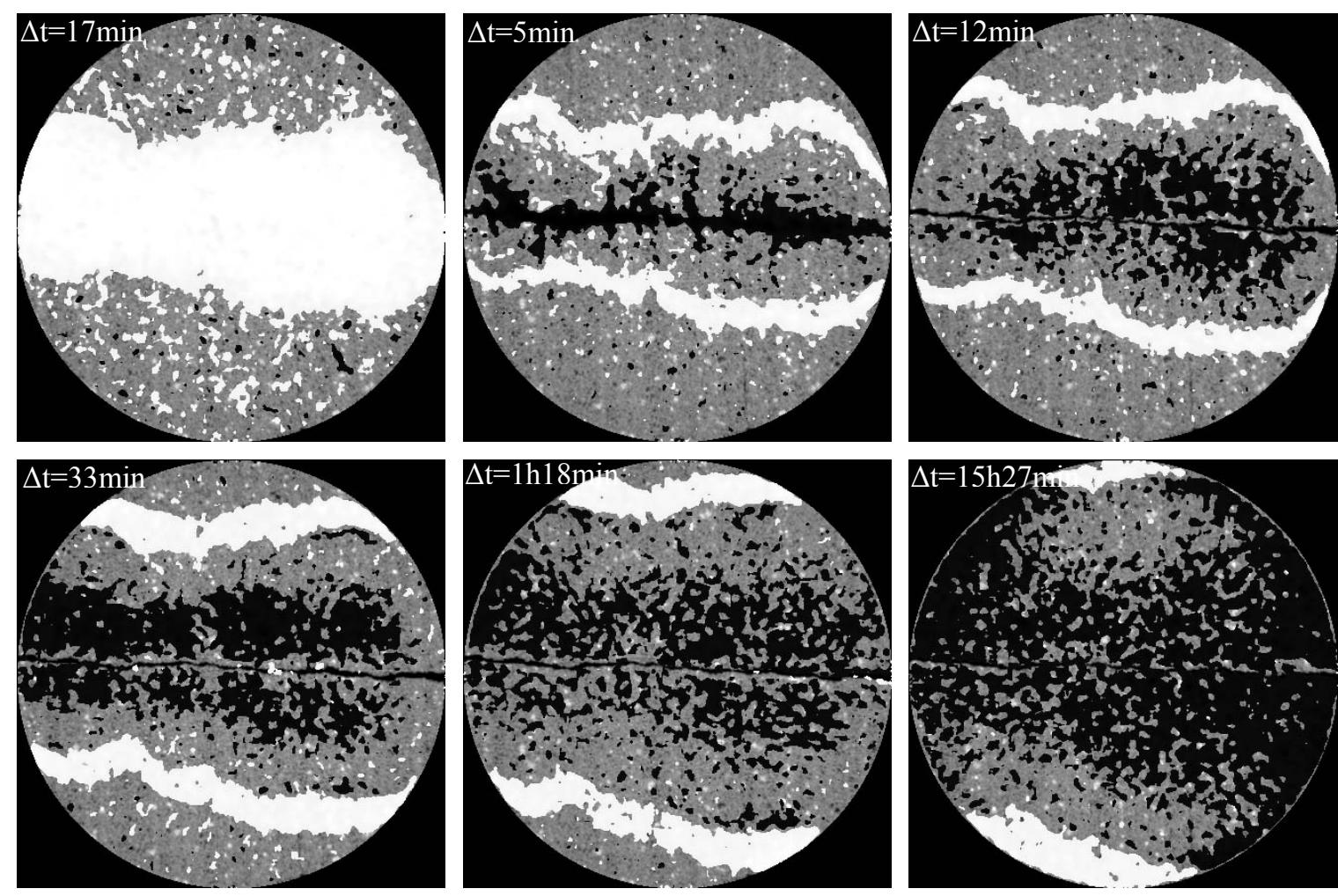

Figure 21: Incremental water saturation during capillary imbibition obtained from highresolution CT scans.

Bedding planes in the matrix determine the shape of the imbibing front. Figure 22 shows a close-up of the 5-minute frame highlighting three layers, and a schematic drawing of those layers. The distribution of imbibed and drained regions in this figure provides evidence of the in-situ flow mechanism. The white water front reaches farther in low permeability layers and it is retarded in high permeability layers. Simultaneously, high permeability layers exhibit a larger drained area in black, which indicates that they supply water to the advancing water front. The imbibing front advances farther through low permeability layers due to higher capillary forces, while cross-layer fluid exchange tends to level the imbibing front. 

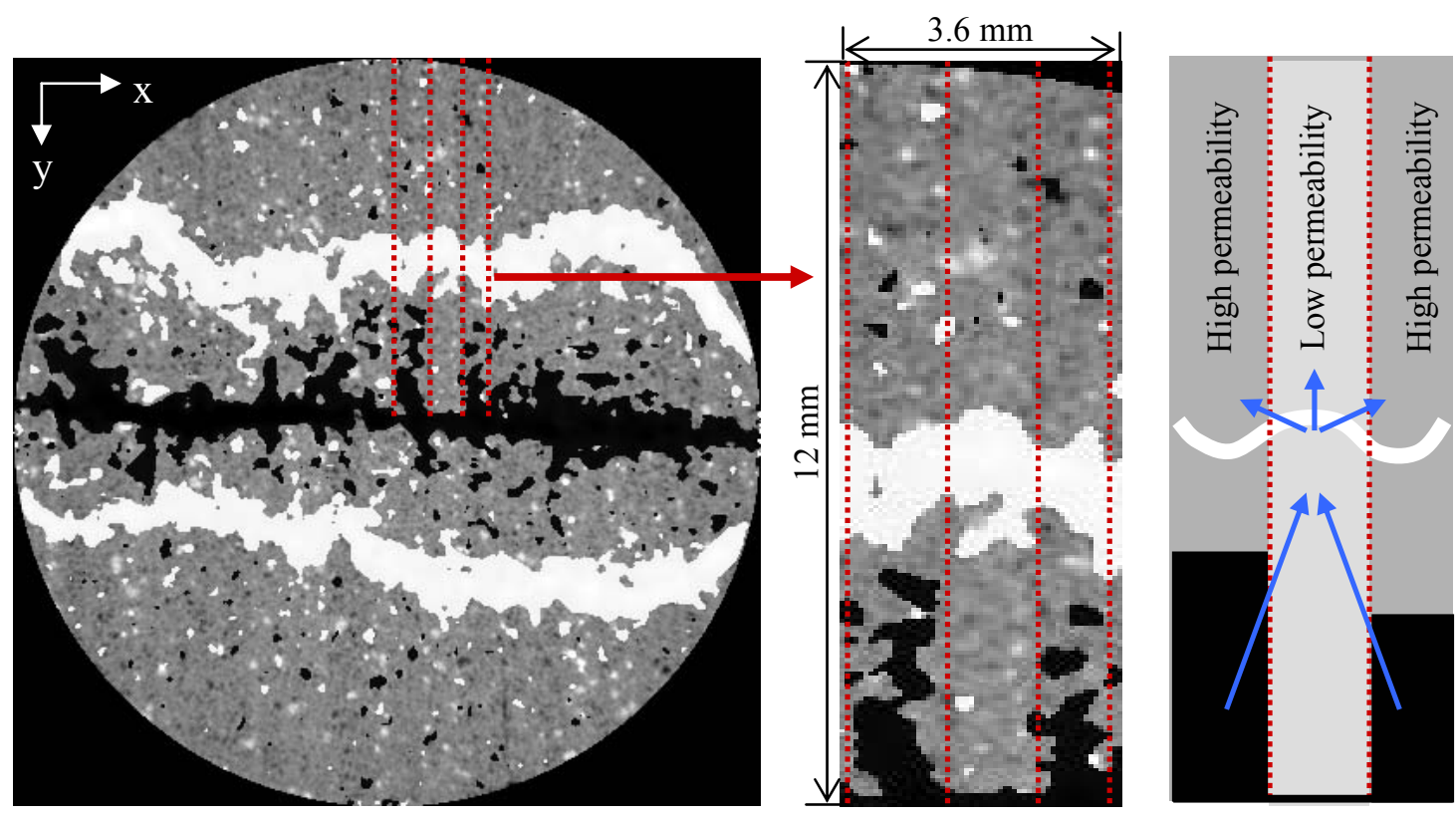

Figure 22: Effect of rock bedding on spontaneous imbibition from subtracted CT scans.

Low porosity/permeability layers in the rock tend to coincide with the narrowest fracture gaps where water is predominantly available, thus promoting advance of the imbibing front through those low permeability layers. The gray transition zone between the black and white contours in Figure 22-left indicates that no significant saturation change occurred during this time period. This transition zone is a major contributor to fluid transport, but its overall saturation shows little-to-no changes.

Saturation profiles were computed over a rectangular portion of the CT slices and averaged in the " $x$ " direction. A schematic representation of this procedure in presented in Figure 23. The resulting profiles capture saturation changes with time in the direction perpendicular to the fracture and parallel to rock layering. 


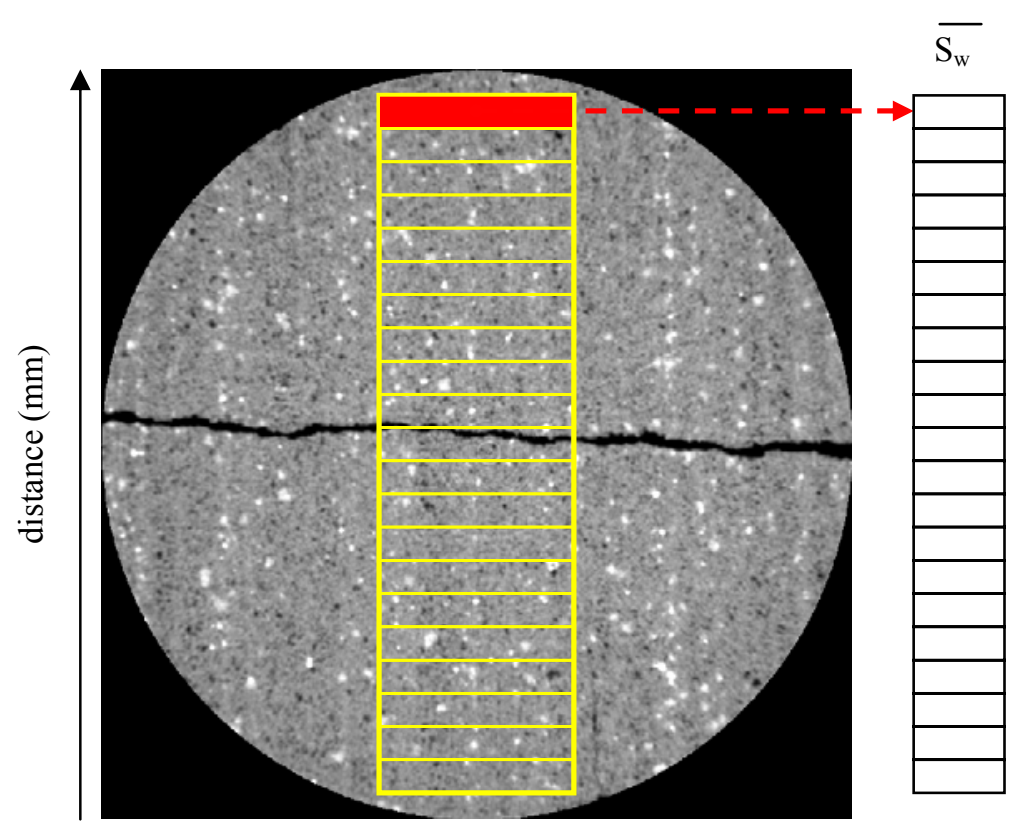

Figure 23: Schematic representation of the CT section used for saturation profile calculation.

The profiles obtained are shown in Figures $\mathbf{2 4}$ and $\mathbf{2 5}$ for cumulative and incremental imbibition fronts. Each of the six profiles in Figures 24 and 25 corresponds to a different time equivalent to those presented in Figures 20 and 21. 


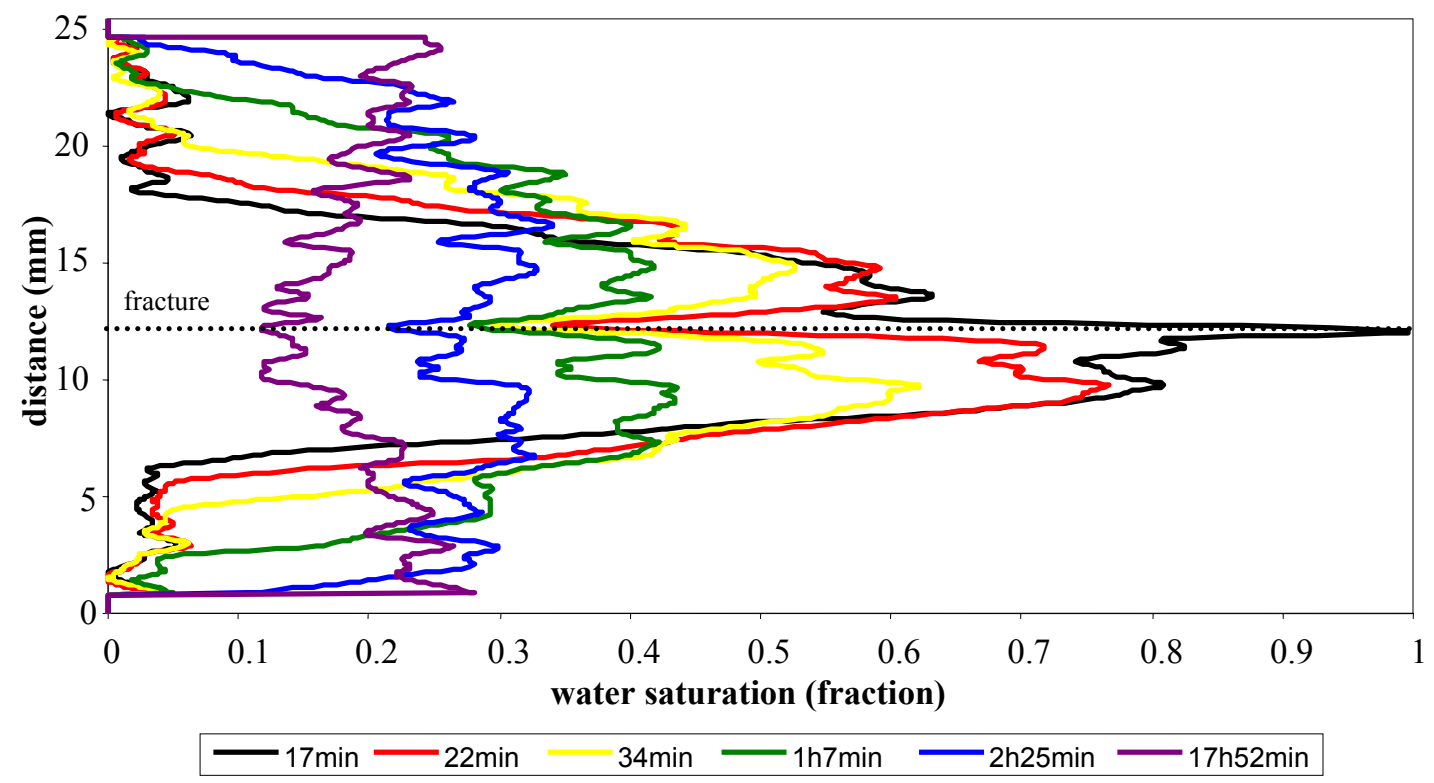

Figure 24: Cumulative water saturation profiles obtained from high-resolution CT scans. Horizontal orientation is $3 \mathrm{~mm}$ above the inlet end of the sample.

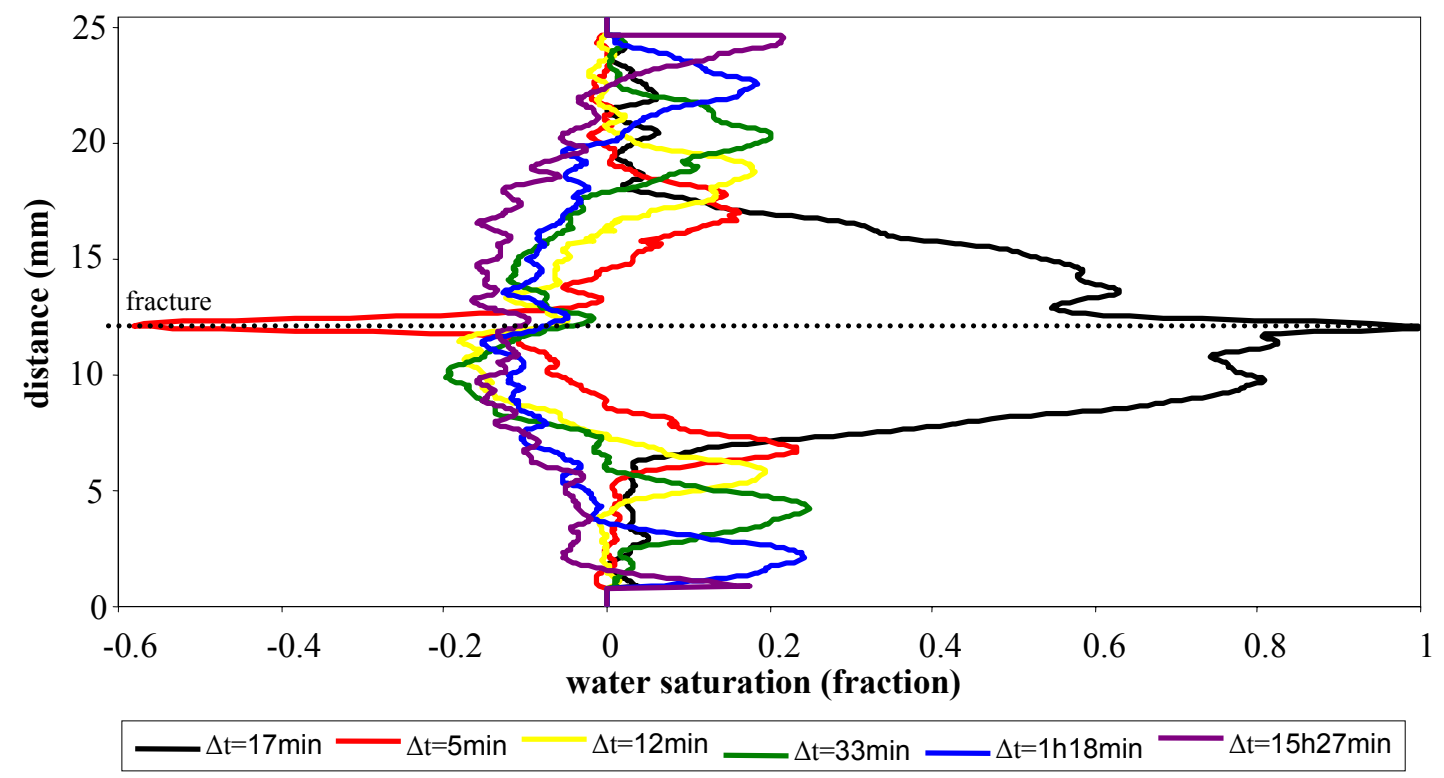

Figure 25: Incremental water saturation profiles obtained from high-resolution CT scans. Horizontal orientation is $3 \mathrm{~mm}$ above the inlet end of the sample. 
The first profile in Figures 24 and 25 (black), obtained after 17 minutes of spontaneous imbibition, shows a high peak of water saturation around $12 \mathrm{~mm}$, which corresponds to the location of the fracture. This saturation peak is an indication of the channeling of water through the fracture gap. Five minutes later, at 22 minutes of cumulative imbibition, water resident in the fractured rapidly flows into the matrix. This mechanism is represented in the saturation profiles by a shift between the black and red lines near the fracture $(12 \mathrm{~mm})$. The red peak is inverted with respect to the black profile for a sharp reduction in water saturation in the fracture. As time progresses, the profiles tend to flatten with water spreading away from the fracture.

Average water saturations for each time profile in Figure 24 were calculated and plotted as a function of time. Results are presented in Figure 26. During early-time imbibition the average water saturation on the scanned slice is close to a constant 0.25 , which suggests that fluid exchange takes place on the scanned horizontal plane. This observation and the symmetry of the imbibing front with respect to the fracture are strong evidence of the prevailing counter-current flow of oil and water near the fracture-matrix interface. However, after 100 minutes of spontaneous imbibition, Figure 20 shows a decrease in average water saturation, which indicates that water in migrating out of the scanning plane, in the direction perpendicular to the CT plane. Thus there must be a balance between early-time counter-current flow near the fracture-matrix interface and longitudinal flow component. 


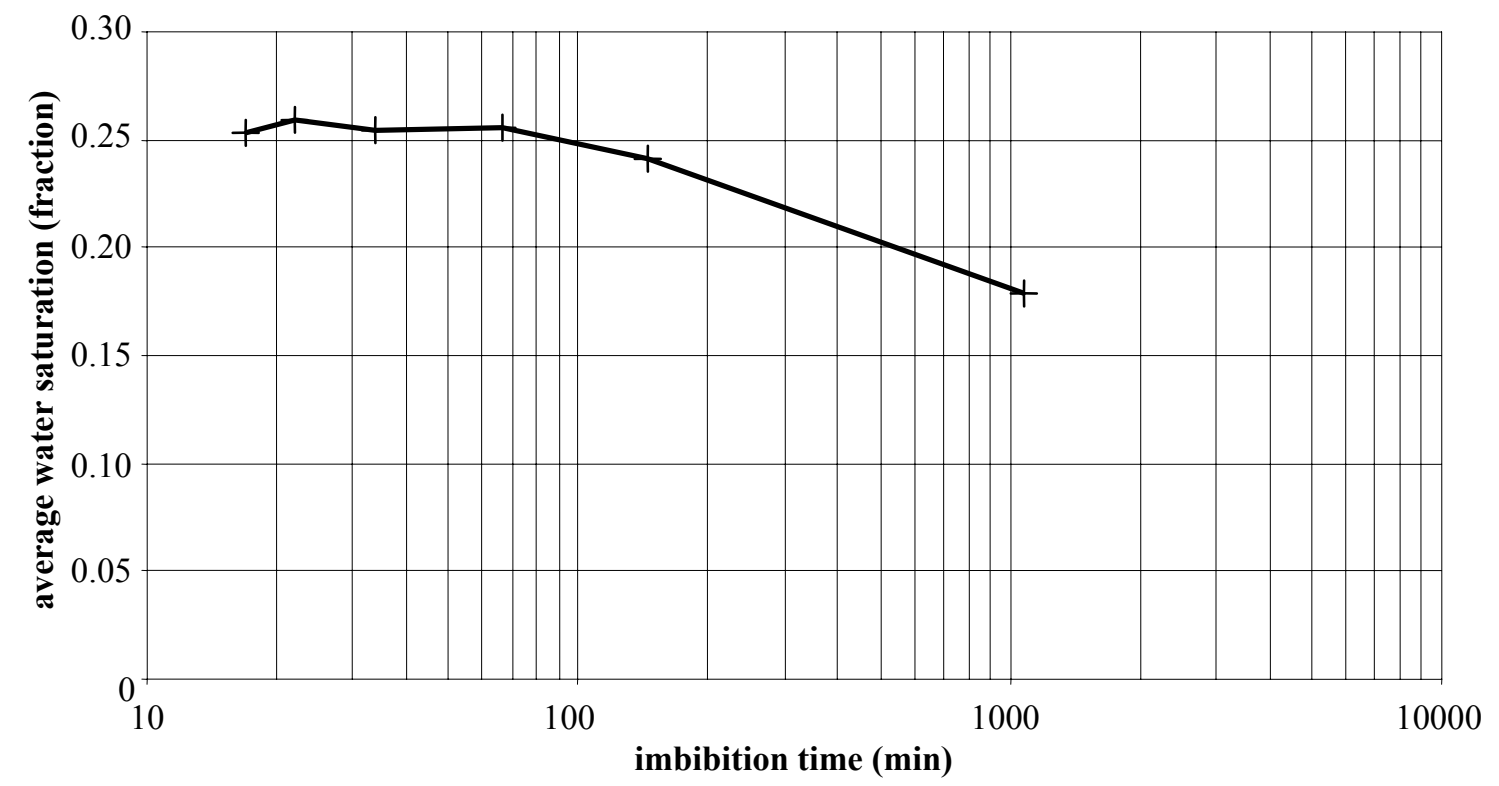

Figure 26: Changes in average water saturation as a function of time for a CT slice $3 \mathrm{~mm}$ above the bottom of the core.

An additional indication of counter-current flow perpendicular to the fracture plane is the presence of saturation banks in the rock matrix. Similar saturation banks have been observed in previous counter-current flow experiments using unconsolidated sands (Briggs and Katz, 1966 and Karpyn, 2001). The presence of saturation banks during two-phase flow in consolidated sandstones has not been previously reported in the literature. Figure 27 shows a threedimensional portion of lower end of the fractured sample that was scanned after 18 hours of spontaneous imbibition. In this figure, a saturation bank is represented by the blue-green halo with low water saturation that is adjacent to the fracture and surrounds pockets of high water saturation (orange-red). 


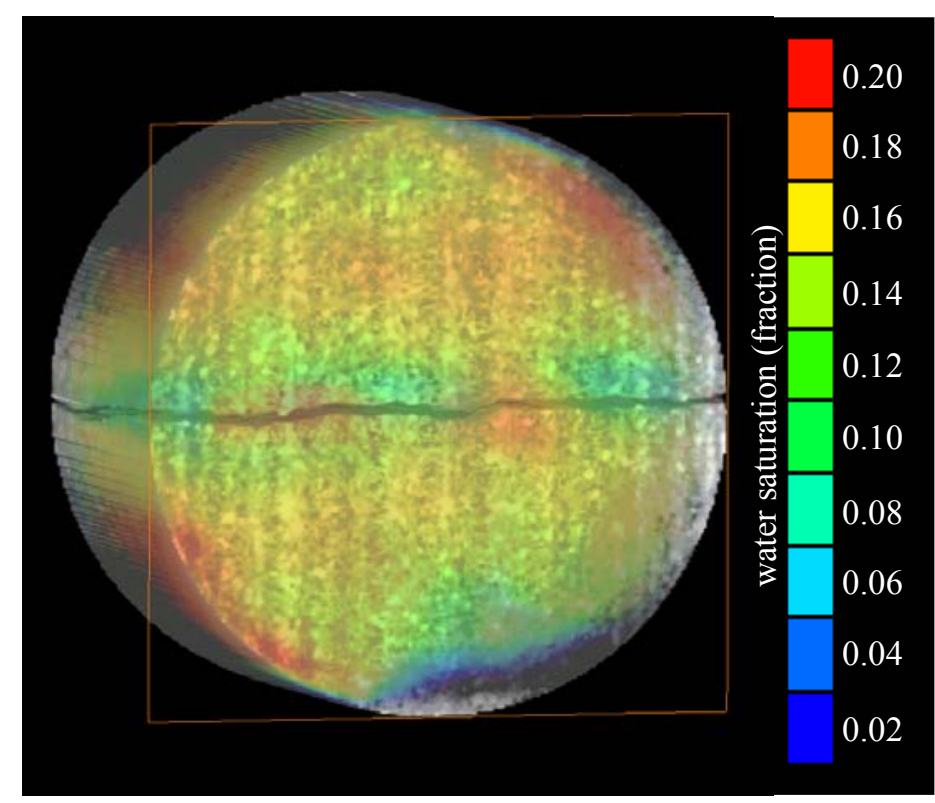

Figure 27: Water saturation map at the end of capillary imbibition 1 showing fluid banks, $1 \mathrm{~mm}$ above the bottom of the core.

Figure 28 also shows a blue halo around the fracture, indicating low water saturation. Low water saturation adjacent to the fracture surface could also be explained by the presence of micro fractures developed during the fracturing process. Micro fractures adjacent to the fracture-matrix interface may be responsible for an increase in local permeability. Previous observations demonstrated that water flows from high permeability layers, which is consistent with the saturation map in Figure 28. 


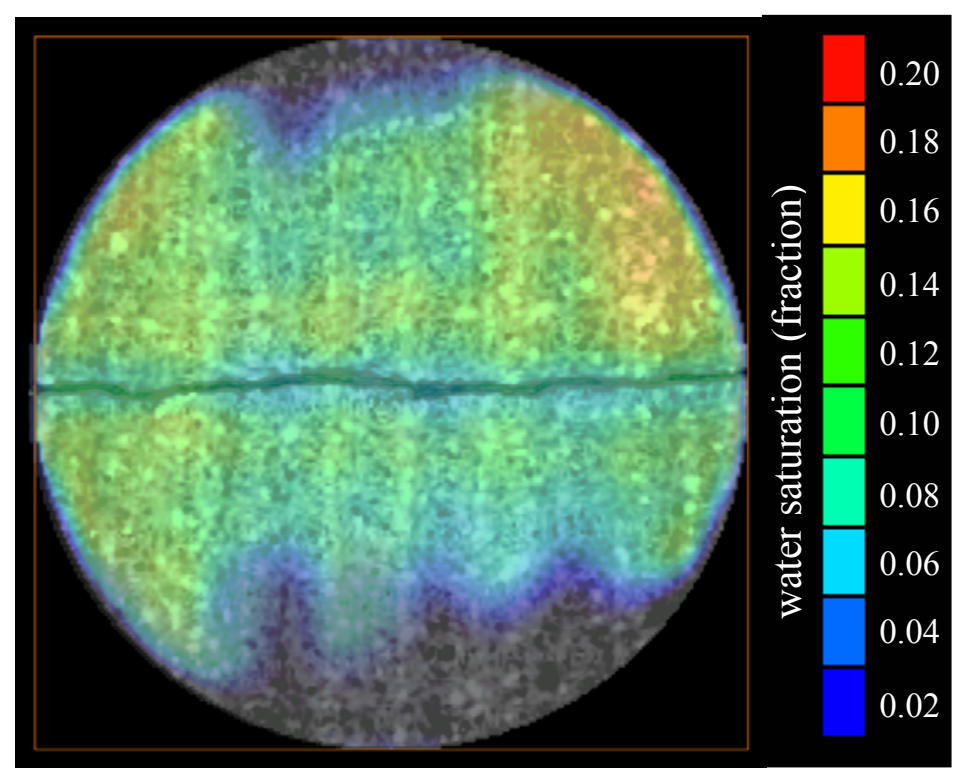

Figure 28: Water saturation map at the end of capillary imbibition 1 showing fluid banks and layering effect, $9 \mathrm{~mm}$ above the bottom of the core.

In summary, non-destructive monitoring of fluid flow in fractured samples allowed visualization of localized co-current and counter-current flow during capillary imbibition. Counter-current flow prevails in the direction perpendicular to the fracture surface, while co-current flow dominates longitudinal imbibition, parallel to the fracture plane. The presence of bedding planes in the rock's structure determines the shape of the imbibing front. The imbibing front advances faster through low porosity/permeability layers due to higher capillary forces, and cross-layer fluid exchange tends to level the imbibing front. These experimental observations are schematically represented in Figure 29. Additional confirmation of these observations was found through numerical simulation of capillary imbibition using a commercial reservoir 
simulation tool. The next stage of the research is to perform conceptual modeling of the experiment in order to understand the fluid flow mechanisms that are taking place.

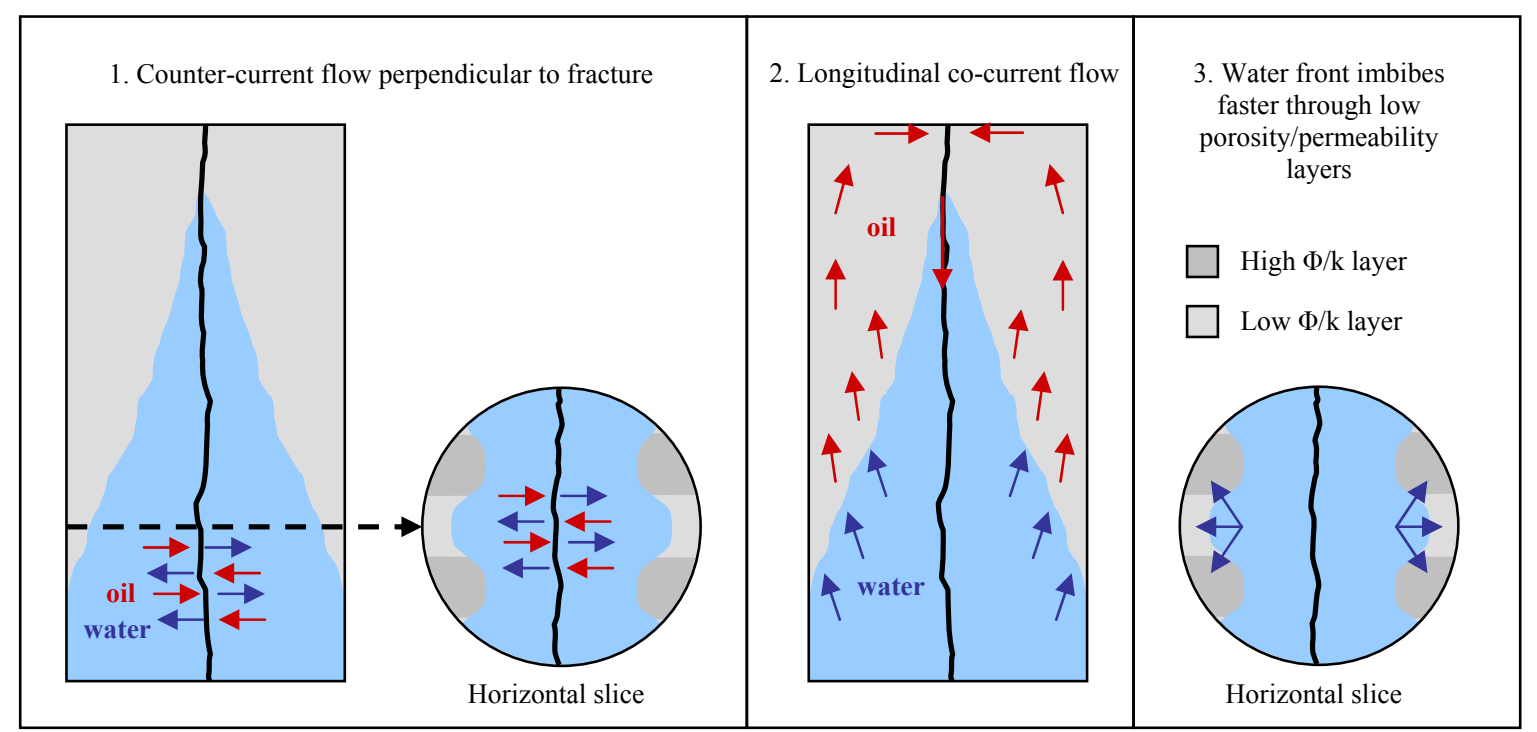

Figure 29: Schematic representation of experimental observations during capillary imbibition. 
Modeling of Counter-Current Imbibition: This experiment focuses on mass transport between the fracture and the matrix under imbibition conditions. The entire core was oil saturated and then a fixed amount of water was injected strictly into the fracture and then allowed to equilibrate. Counter-current imbibition created water flow from the fracture to the matrix and oil flow from the matrix to the fracture. This is an essential process to understand and quantify as it is the main displacement production mechanism that allows us to extract hydrocarbons from matrix blocks into the transmitting fractured network. In this section, conceptual modeling of water imbibing into the matrix from the fracture, and the counter-flow of oil into the fracture are considered. The experimental system was described in the previous report, and is not presented here.

Reservoir simulation at laboratory scale was implemented to assist in understanding various experimental observations and flow mechanisms taking place during capillary imbibition in fractured media. The simulation packages used were developed by Computer Modeling Group, Inc. (CMG). The specific tools used were Builder for pre-processing applications, Results for post-processing applications, and IMEX: IMplicit-EXplicit Black Oil Simulator for the actual two-phase model. The input files created are presented in Appendix B of the seventh semiannual report.

The first case study consisted of a two-dimensional vertical reservoir with dimensions $12.5 \mathrm{~mm} \mathrm{x}$ $48 \mathrm{~mm}$, representing one half of the system. The sample radius was approximately $12.5 \mathrm{~mm}$ and its height $48 \mathrm{~mm}$, which were selected as the reservoir dimensions. Figure 30 shows the grid used to represent fracture and matrix in this model. The grid has 13 blocks in the "i" direction 
(horizontal) and 48 blocks in the "j" direction (vertical). The blue column represents the fracture and the grid blocks in red are matrix blocks. The dimensions of each fracture blocks was $0.5 \mathrm{x} 1$ x $1 \mathrm{~mm}$ and the dimensions of each matrix block was $1 \times 1 \times 1 \mathrm{~mm}$.
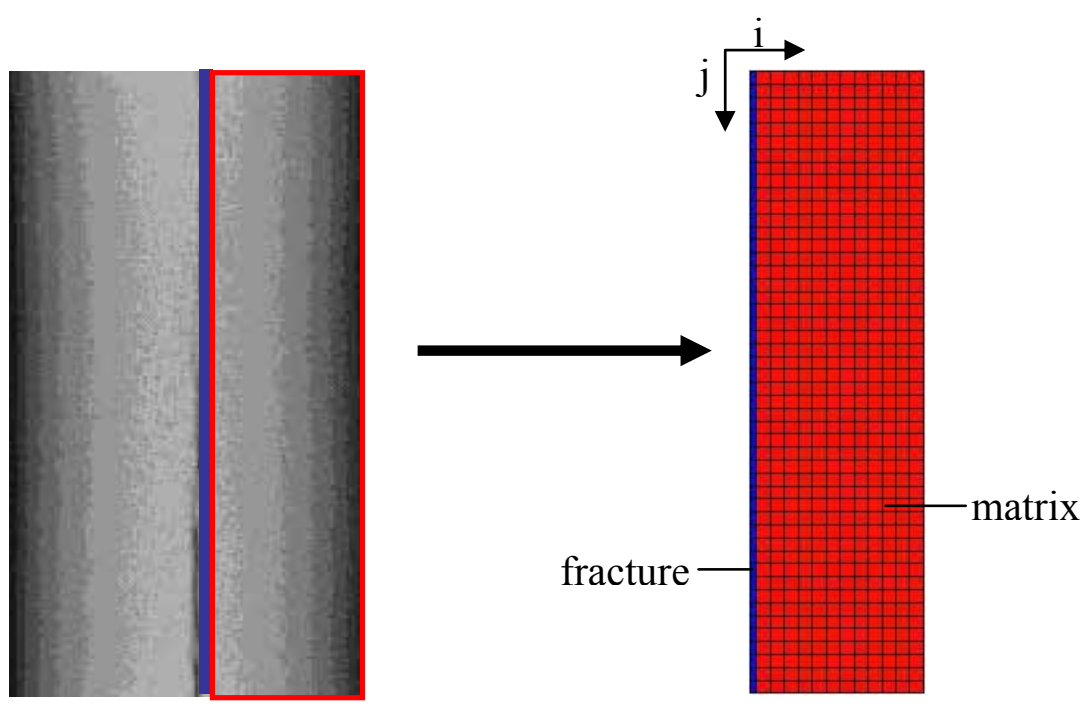

Figure 30: Grid model for two-dimensional imbibition.

Rock and fluid properties were estimated from those reported by Alajmi (2003), who conducted numerical history matching of two-phase flow experiments in fractured Berea samples, using similar fluids and rock. Alajmi (2003) used 64 md of absolute permeability in all directions and an average porosity of $17.5 \%$ in his two-dimensional model. Same values were used in the current two-dimensional model. Fracture porosity was calculated from the ratio between the actual volume of the fracture, obtained from CT data, and the bulk volume of the simulated fracture, denoted in blue in Figure 30. A volume equivalent to the simulated two-dimensional 
grid was identified on the CT data and used to calculate the actual volume of the fracture. Table 1 presents a summary of porosity and permeability values used in the present twodimensional model.

\begin{tabular}{|c|c|}
\hline \multicolumn{2}{|c|}{ Table 1: Rock properties assigned to fracture and matrix in two- } \\
dimensional model. \\
\hline PROPERTY & VALUE \\
\hline Fracture permeability [md] & 5,000 (isotropic) \\
\hline Matrix permeability [md] & 64 (isotropic) \\
\hline Fracture porosity [fraction] & 0.38 \\
\hline Matrix porosity [fraction] & 0.175 \\
\hline
\end{tabular}

Capillary pressure and relative permeability curves used in the two-phase imbibition model are presented in Figures 31 and 32. The curves assigned to the matrix are similar to those presented by Alajmi (2003). Residual oil saturation in the fracture is larger than that in the matrix due to oil trapping in the fracture.

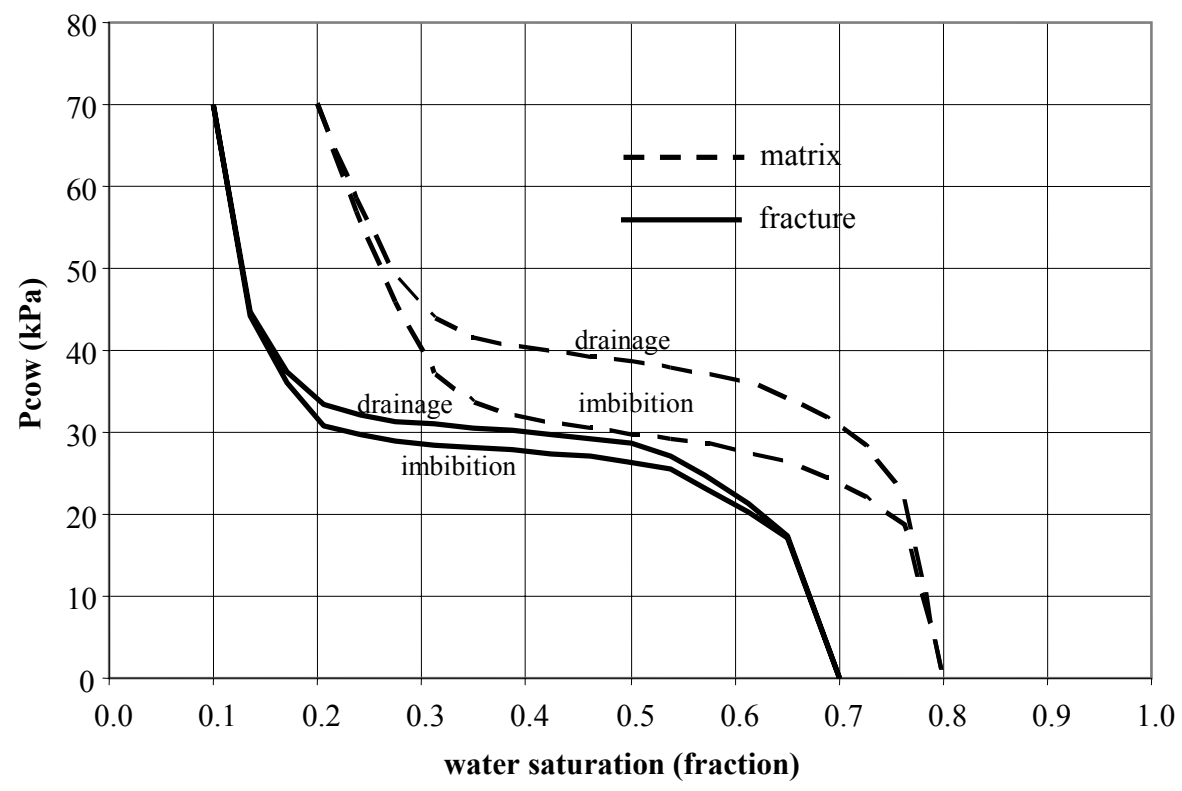

Figure 31: Capillary pressure curves used for two-dimensional model. 


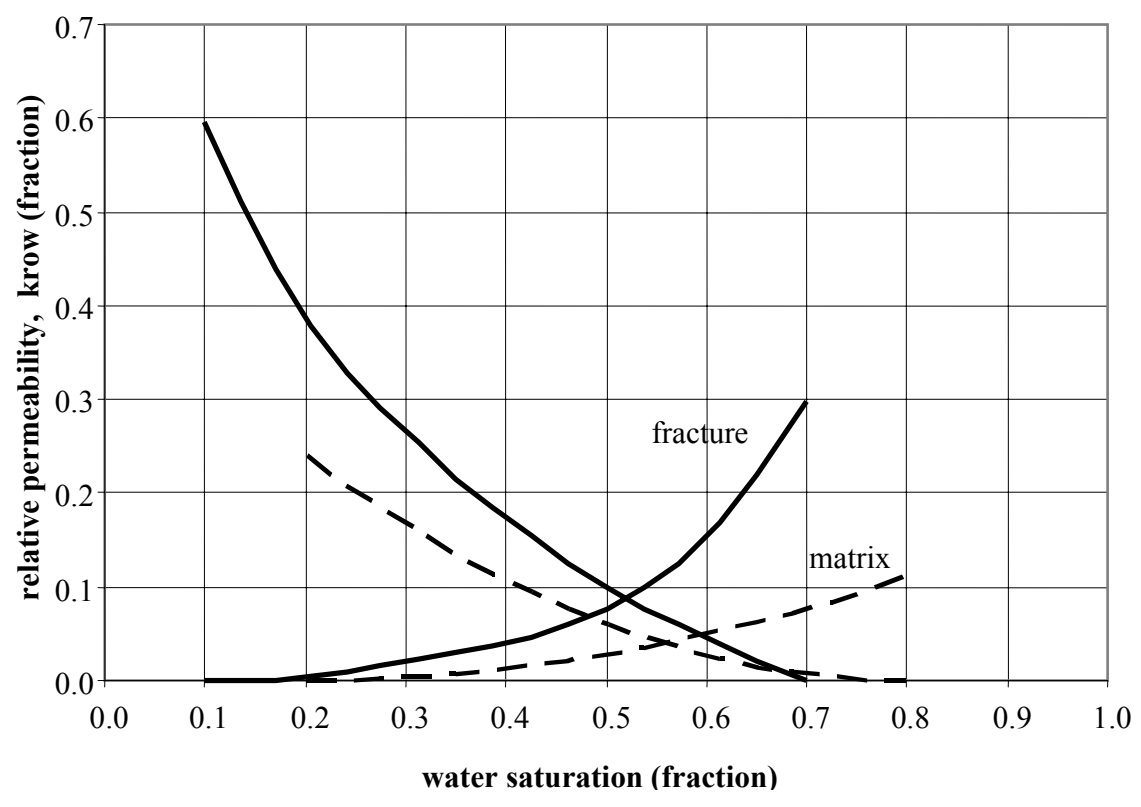

Figure 32: Relative permeability curves used for numerical modeling.

Initially, fracture capillary pressures were turned off in the two-dimensional model and only matrix capillary pressures were accounted for. As a result, simulated saturation maps were far from capturing the real imbibition process. Figure 33 shows the simulated maps using fracture capillary pressure equal to zero. The simulated water front is significantly shorter than the one in the digital radiographies. The imbibing front in the experimental maps was able to reach much farther than what the model could predict. The discrepancy between experiments and simulation suggests that an additional driving force is elevating the water through the fracture sample, just as capillarity would do. 


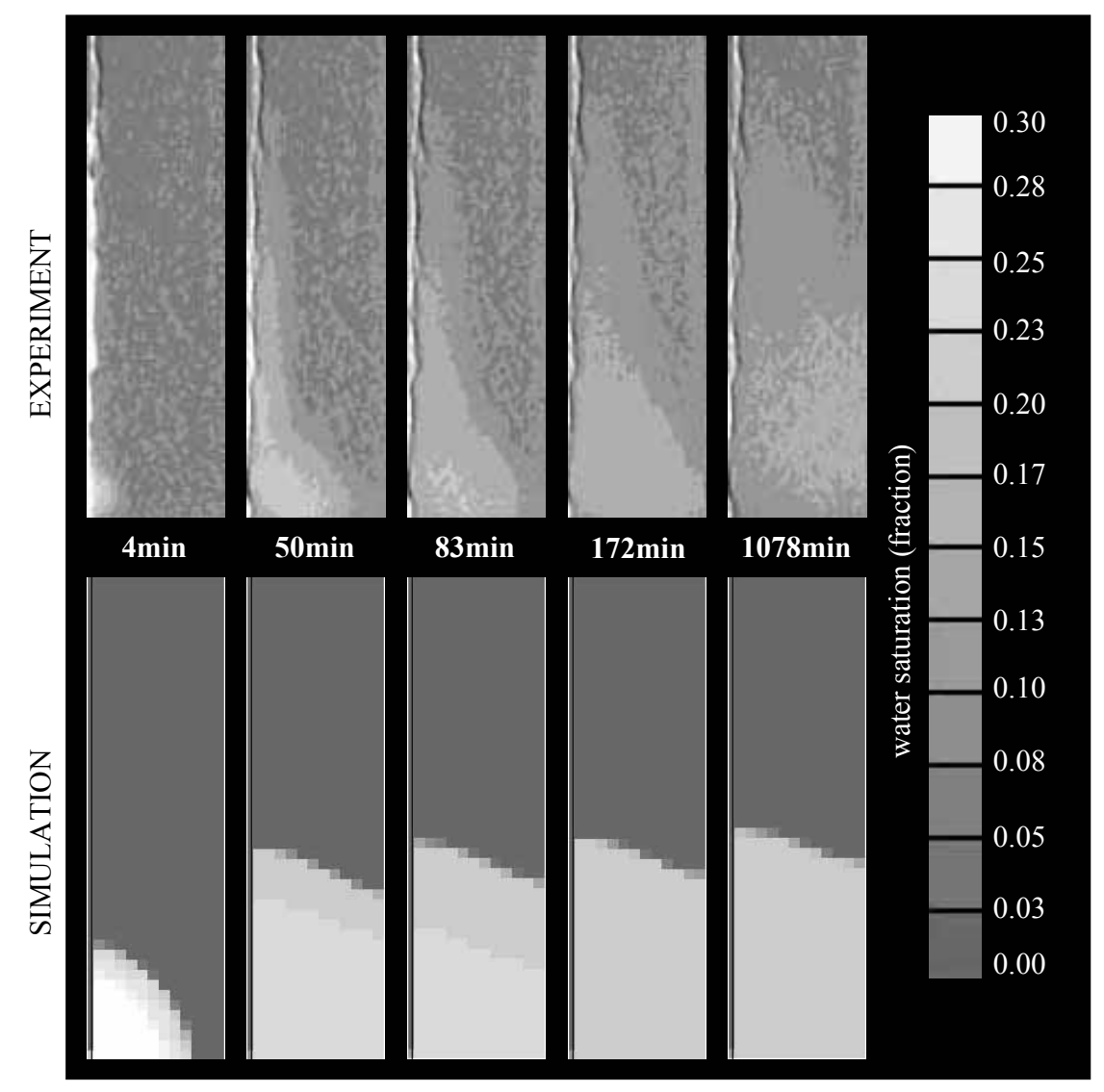

Figure 33: Simulated water front using fracture capillary pressure equal to zero.

A second case scenario was tested where both fracture and matrix had the same assigned capillary pressure curves. This simulated scenario delivered the saturation maps presented in Figure 34.

A comparison between simulated saturation maps in Figures 33 and $\mathbf{3 4}$ demonstrated strong sensitivity with respect to fracture capillary pressure. Fracture capillary pressure is often regarded as negligible in fractured reservoir simulations, but experimental observation has proven otherwise. Fracture capillary pressures are a major contributor to flow, and their impact must not be overlooked by the modeler. Accounting for capillary forces in the fracture is essential for successful modeling of fracture-matrix flow. Contact areas in the fracture provide 
passages under strong capillary action, which are able to drive wetting fluids into the rock matrix. Such behavior can be captured by means of fracture capillary pressures and void structure. Water available to the fracture immediately reaches for the narrowest gaps and imbibes the rock matrix near contact areas. Fracture capillary forces are especially important when the rate of injection is close to or less than the matrix's capacity to transport fluids.

A third modification on the fracture capillary pressure curve led to the results presented in Figure 35. In this case, fracture capillary pressures assigned to the model were lower than those assigned to the matrix. For most part of the mobile saturation window, matrix capillary forces are higher than those in the fracture.

Capillary-driven flow in fractured media and the mechanism by which oil and water exchange places is governed by aperture distribution, capillarity, permeability and wettability. They compete to guide fluid flow between fracture and matrix, and through the different layers of the rock. One of the hypotheses posed in this work was that capillary-driven imbibition would take place mainly due to local counter-current flow of wetting and non-wetting phases. However, experimental observations suggest that counter-current flow prevails in the direction perpendicular to the fracture surface, while co-current flow dominates longitudinal imbibition, parallel to the fracture plane. 


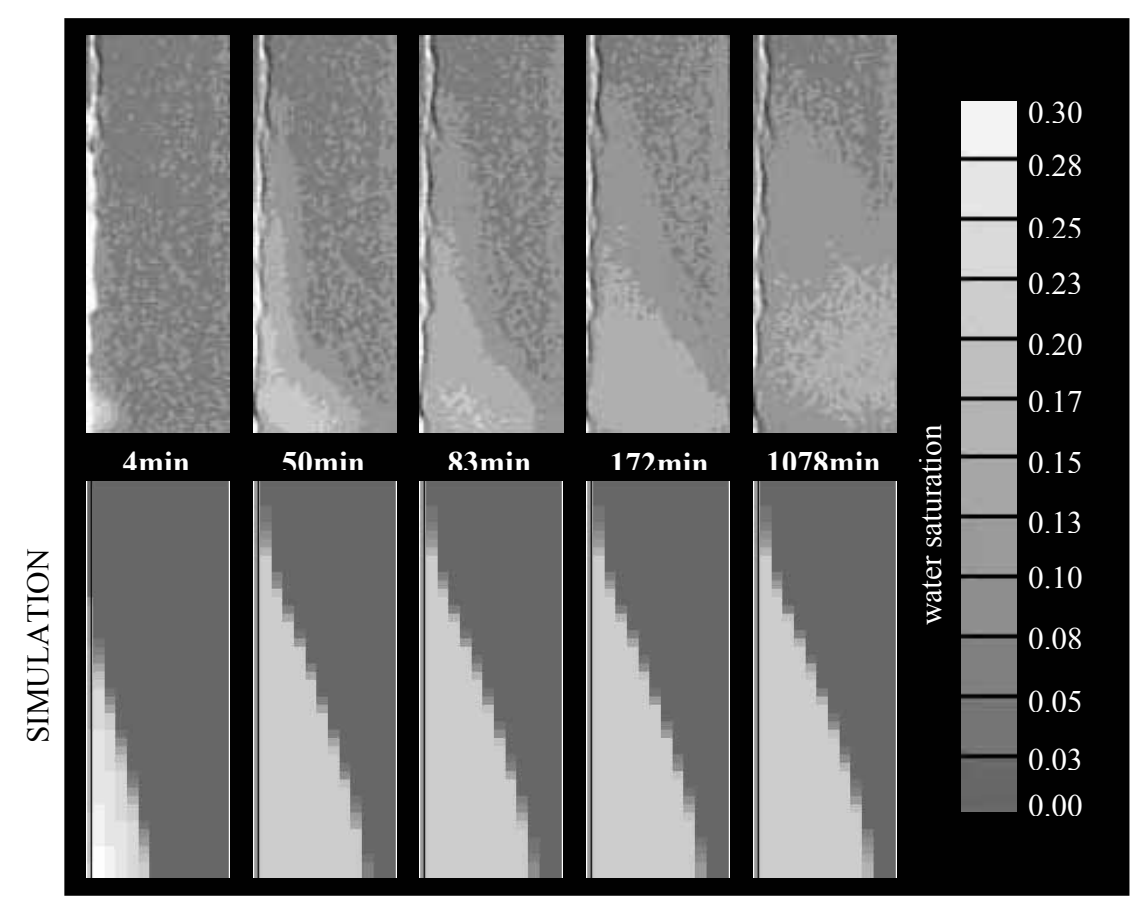

Figure 34: Simulated cumulative water front using similar capillary pressure curves in the fracture and the matrix. 


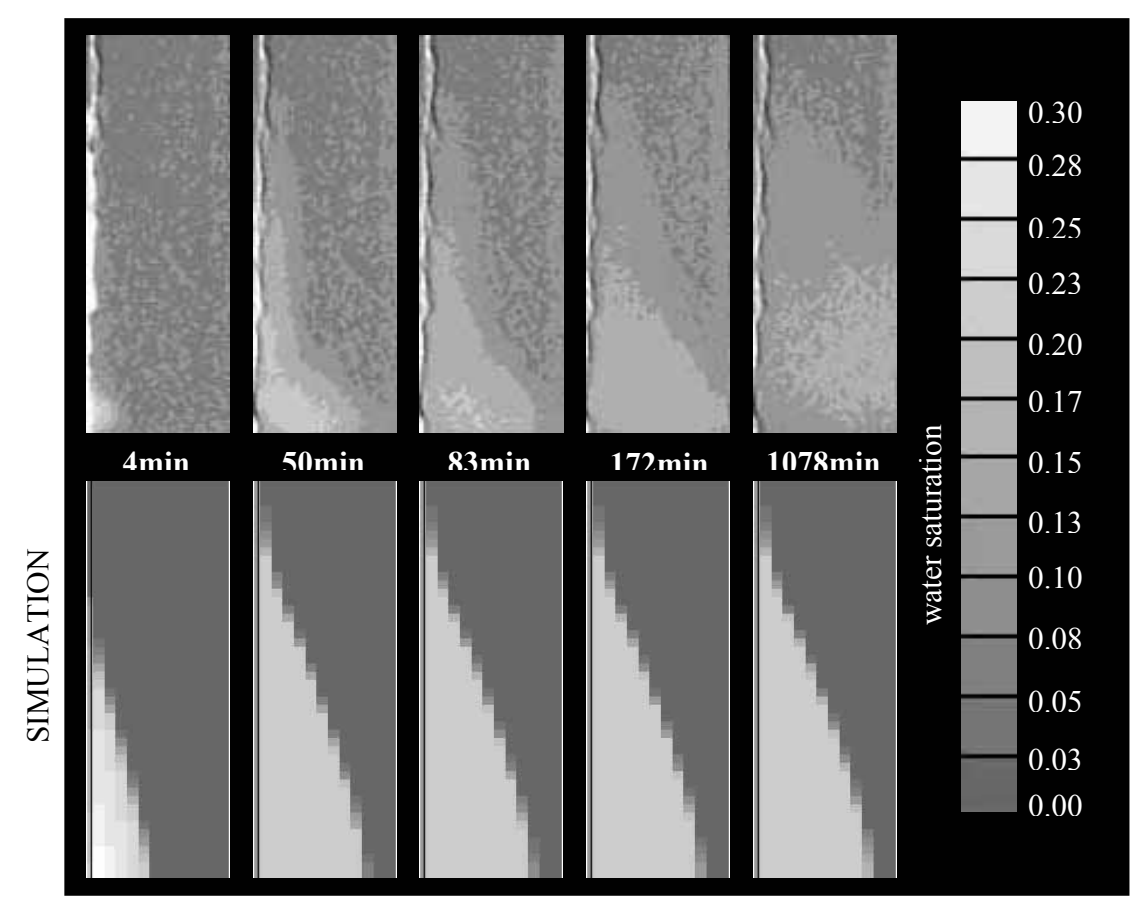

Figure 35: Simulated cumulative water front obtained from two-dimensional two-phase imbibition model.

A synthetic three-dimensional model was created using the black-oil reservoir simulator developed by CMG. The model represents a fractured system with two layers with different rock properties. Figure 36 shows the grid used to represent fracture and matrix in this model. The size of this three-dimensional system is $20 \times 23 \times 20 \mathrm{~mm}^{3}$, with individual grid blocks of $1 \times 1 \times 1$ mm each. 


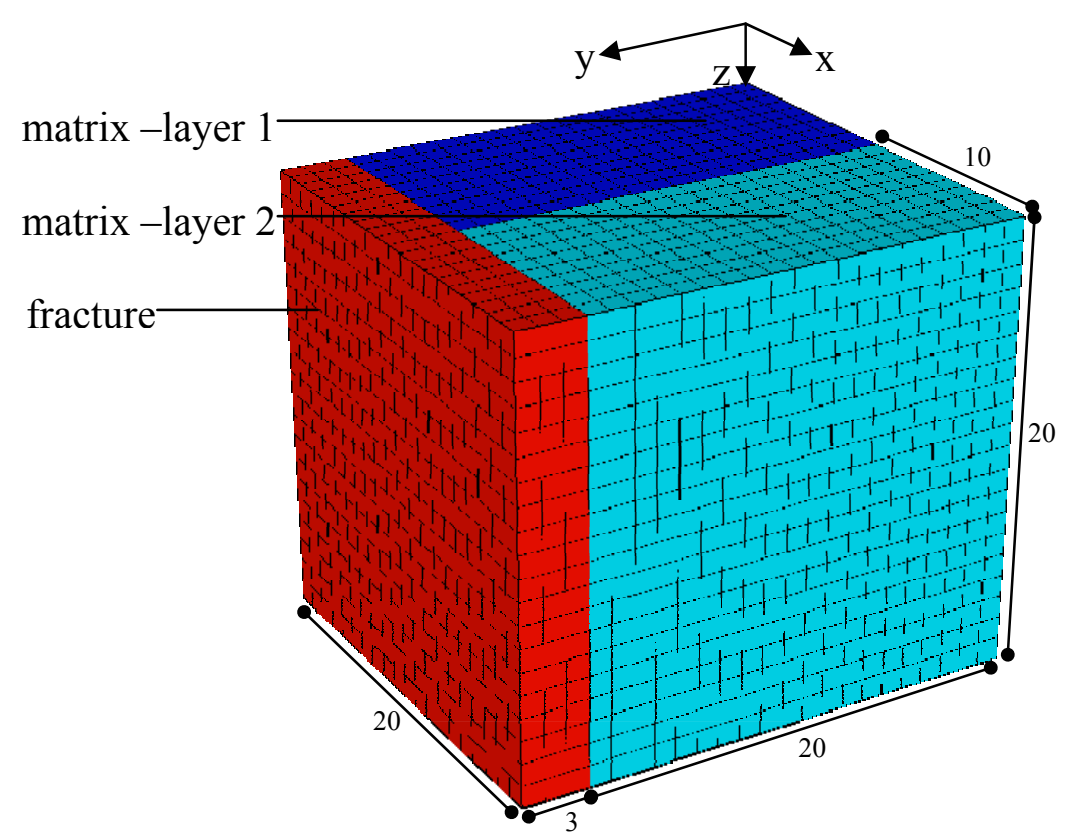

Figure 36: Grid model for simulating three-dimensional imbibition.

Rock properties used in this three-dimensional model were initially estimated from typical values found in layered Berea samples. However, those properties were later modified to enhance visualization of the flow mechanism taking place. The final rock properties used in this threedimensional model are listed in Table 2. 


\begin{tabular}{|c|c|}
\hline \multicolumn{2}{|c|}{$\begin{array}{l}\text { Table 2: Rock properties assigned to fracture and matrix in three-dimensiona } \\
\qquad \text { model. }\end{array}$} \\
\hline PROPERTY & VALUE \\
\hline Fracture permeability [md] & 500 (isotropic) \\
\hline Matrix permeability - layer $1[\mathrm{md}]$ & 40 (isotropic) \\
\hline Matrix permeability - layer $2[\mathrm{md}]$ & 60 (isotropic) \\
\hline Fracture porosity [fraction] & 0.38 \\
\hline Matrix porosity - layer 1 [fraction] & 0.12 \\
\hline Matrix porosity - layer 2 [fraction] & 0.18 \\
\hline
\end{tabular}

Different capillary pressure curves were assigned to layers 1 and 2 in the matrix. The low porosity/permeability layer (1) has higher capillary pressure values than the high porosity/permeability layer (2). Both layers 1 and 2 have higher capillary pressure values than the fracture. Figure 37 shows the capillary pressure curves used in the three-dimensional model. The drainage capillary pressure curve in each case corresponds to the upper line in the capillary loop, while the lower line corresponds to imbibition. The relative permeability curves used were similar to those presented in Figure 32.

At initial conditions, the two layers in the matrix were fully saturated with oil, while water saturation increases from top to bottom in the fracture blocks. The model was subject to spontaneous mobilization of fluids and no injection took place at any time. Fracture blocks at the top of the structure have $50 \%$ water saturation, and blocks at the bottom of the sample have $100 \%$ water saturation. Experimentally, the sample had more water available at the bottom due 
to upward water injection. Changes in water saturation as a function of time obtained from the three-dimensional model are presented in Figure 38. Water rapidly flows into the rock matrix, reaching farther through the low permeability layer. At early time, most fluid exchange takes place near the fracture-matrix interface, while cross-layer and in-layer flow become dominant when the fracture has drained. Figure 9 shows the development of water saturation on three faces of the model.

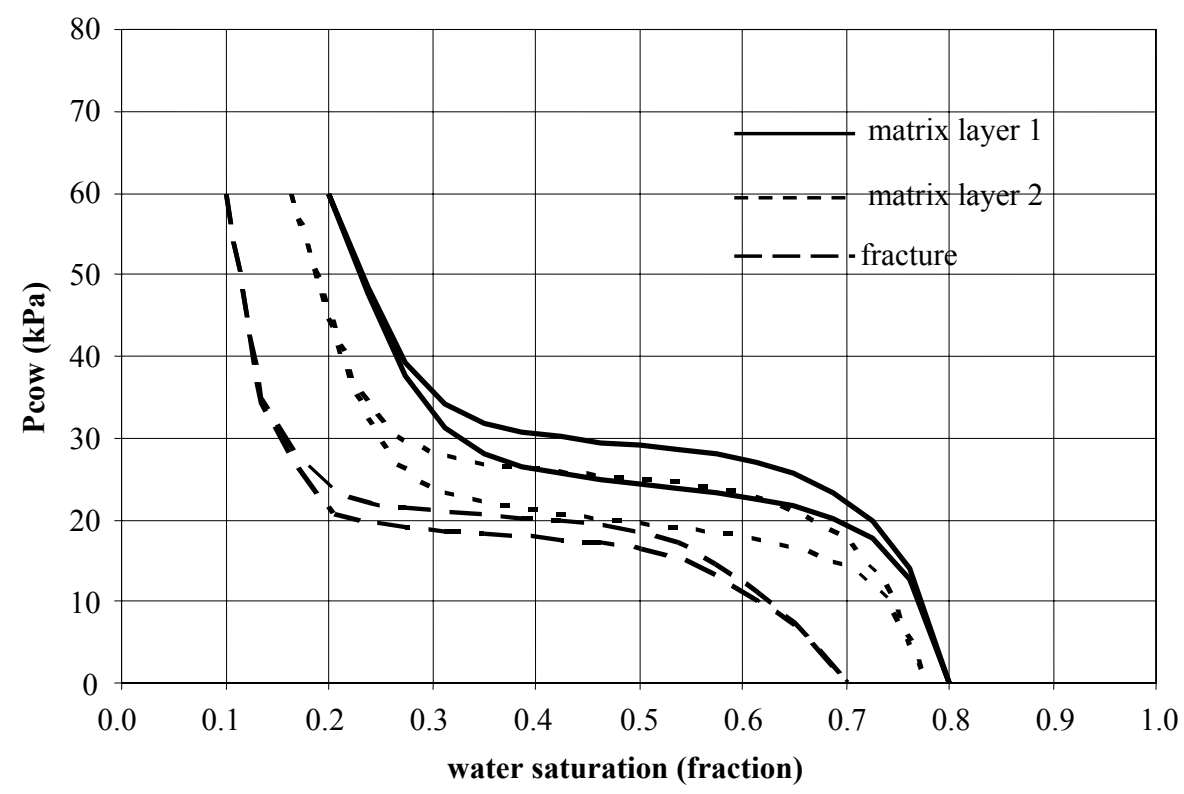

Figure 37: Capillary pressure curves used in the three-dimensional model. 


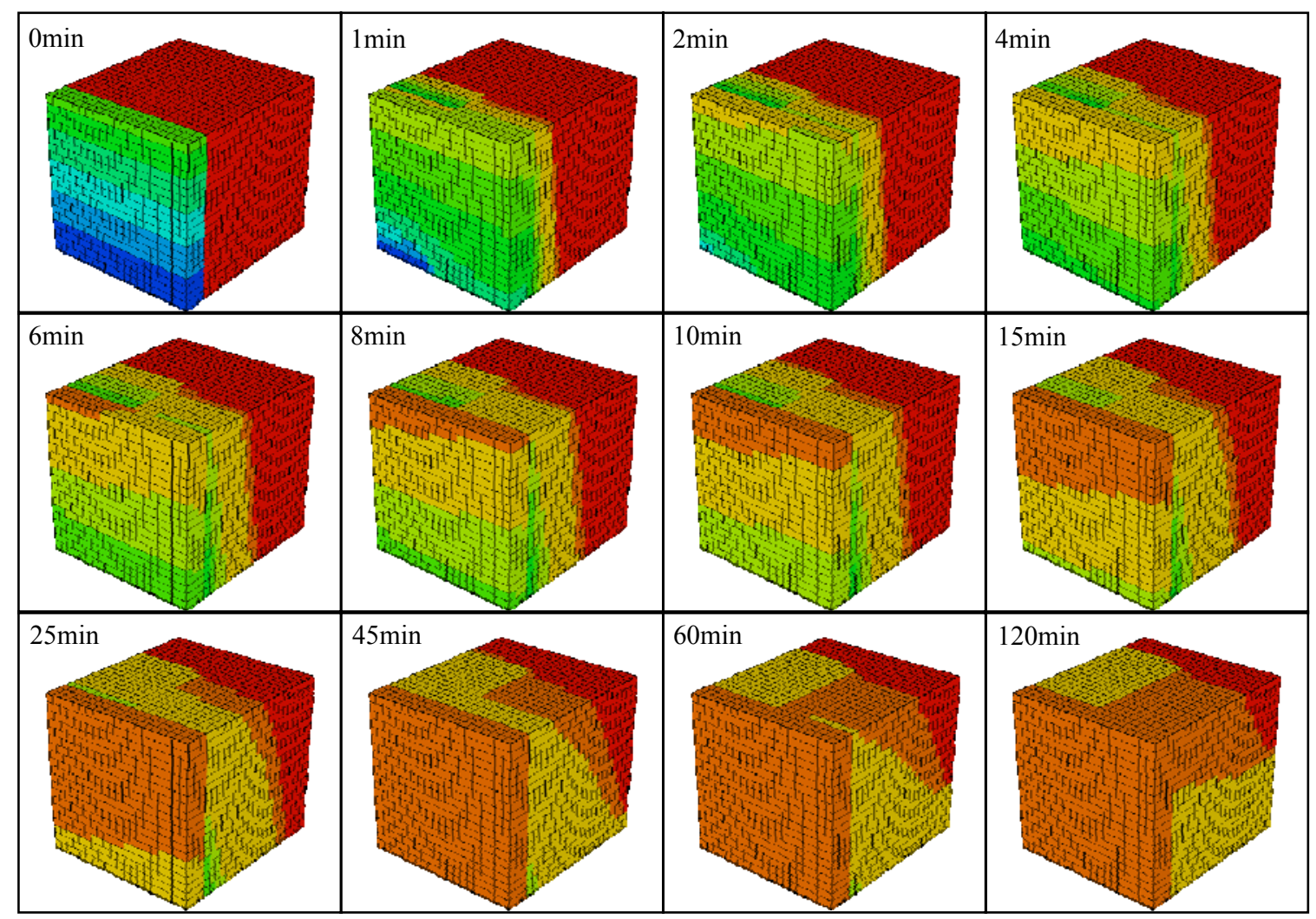

Figure 38: Changes in water saturation during capillary imbibition obtained from threedimensional model.

Different cross-sections of the three-dimensional model were analyzed individually. Figure 39 shows the $x-y$ or axial cross-section with the corresponding water saturation changes. The axial plane was located at $\mathrm{z}=11$, penetrating the two matrix layers and perpendicular to the fracture plane. The axial cross-section shows rapid water advance through the low porosity/permeability layer and a smooth transition of the water front between the two layers. 


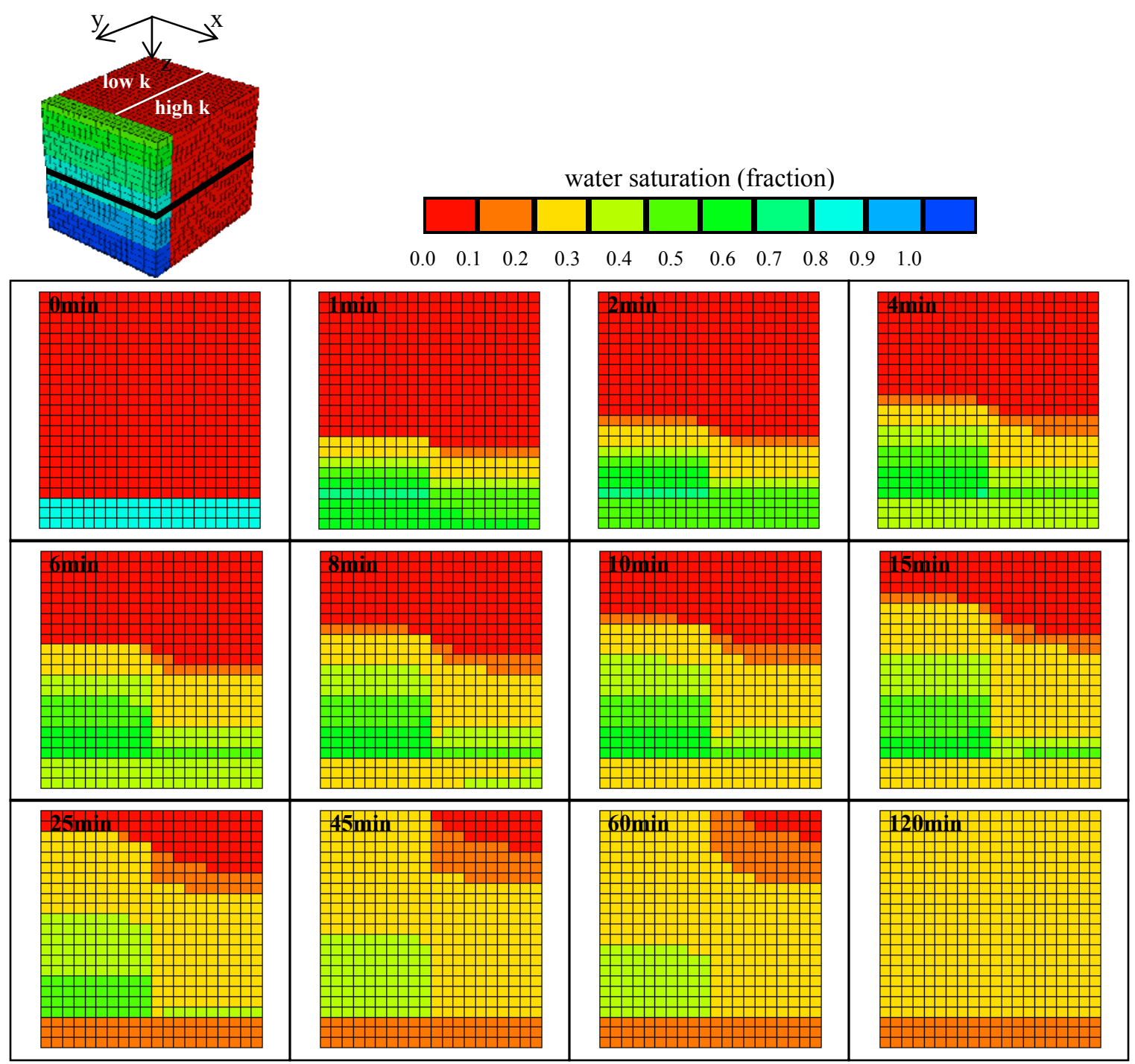

Figure 39: Water saturation changes in an axial cross-section, slice 11.

Figure 40 shows the same axial cross-section at two different times with velocity vectors for oil and water as they exchange places in the reservoir model. The top two snapshots have water flow vectors, while the bottom two have oil flow vectors. Water flow vectors at 4 minutes (topleft in Figure 40) are all pointing towards the matrix with an additional flow component between the two layers and with the fracture. Water preferentially flows from the fracture into the low permeability layer (left) and then from the low to the high permeability layer (right). Water flow vectors at 90 minutes (top-right) shows the same mechanism that explains why the water front in the high permeability layer is delayed with respect to the low permeability layer. The snapshots 
at the bottom of Figure $\mathbf{4 0}$ show how the oil phase flows towards the fracture in counter-current flow with the water phase. Additionally, preferential flow of water through the low permeability layer pushed the oil ahead of the imbibing front, which returns in a cyclic motion through the high permeability layer and to the fracture.
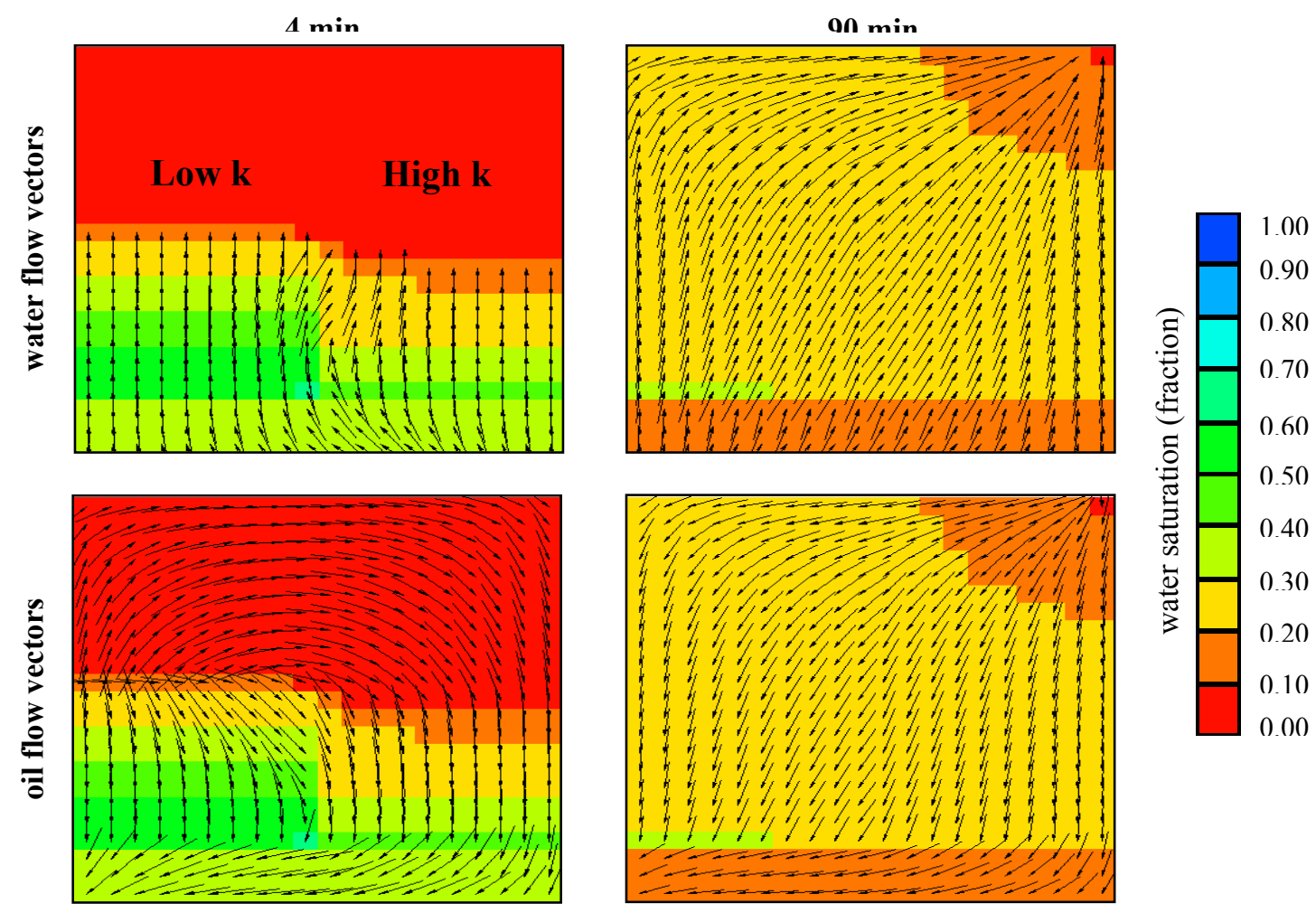

Figure 40: Water and oil flow vectors on axial cross-section at 4 and 90 minutes, slice $\mathrm{z}=11$. 
The simulated axial cross-sections confirmed important experimental observations explaining the mechanism of water imbibition in a fractured system with two matrix layers (see items 1 and 3 in Figure 2). Figure 41 shows the $x-z$ or coronal cross-section with the corresponding water saturation changes. The coronal plane was located at $\mathrm{y}=11$, penetrating the two matrix layers and parallel to the fracture plane. The coronal cross-section at 4 minutes shows the arrival of the imbibing front through the low permeability layer (left). Fluid equilibration becomes slower

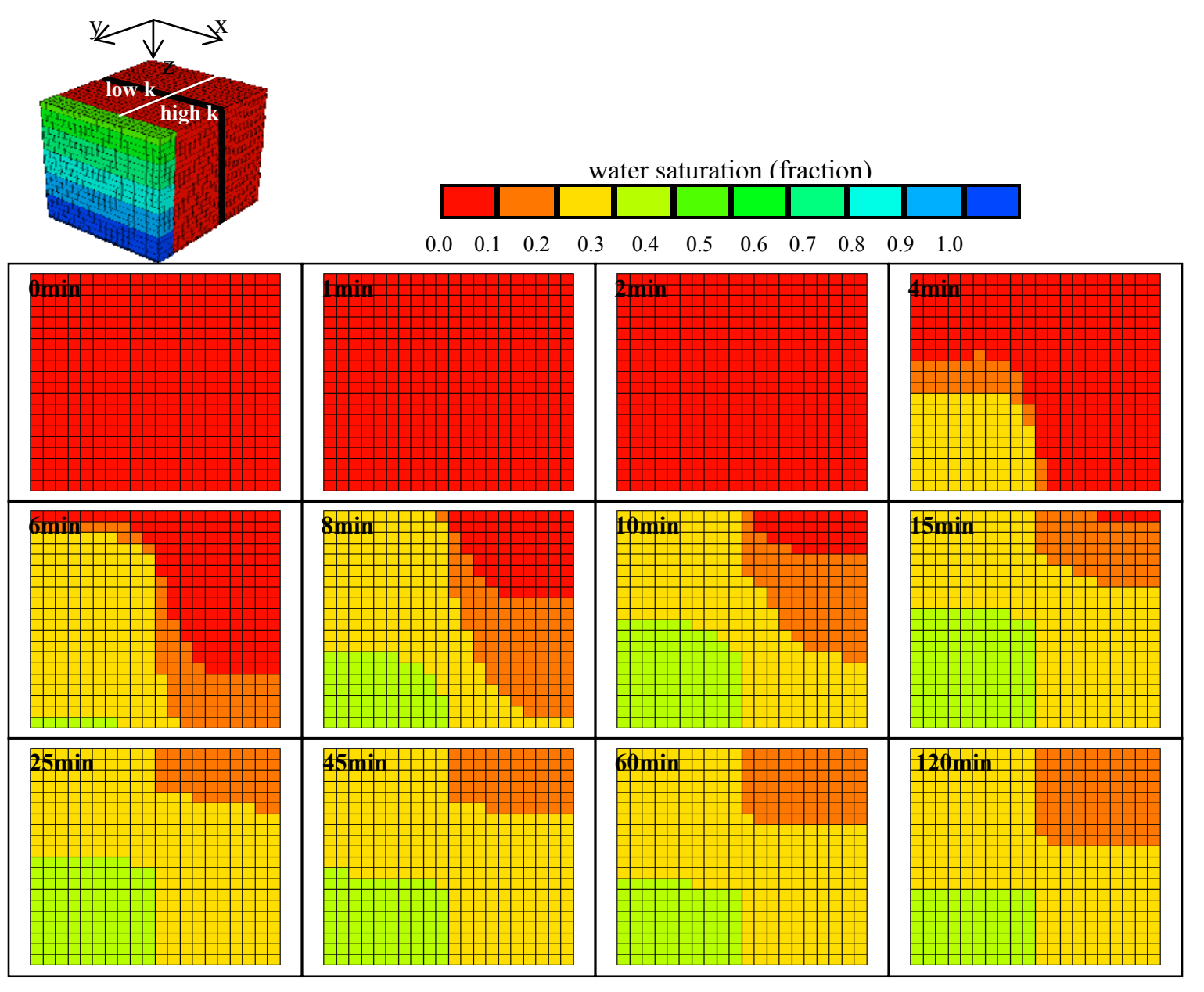

with time due to small er satur ation gradi ents drivi ng the flow mechanism.

Figure 41: Water saturation changes in a coronal cross-section, slice $y=11$. 
Figure 42 shows the same coronal cross-section at two different times with velocity vectors for oil and water as they exchange places in the reservoir model. The top two snapshots have water flow vectors, while the bottom two have oil flow vectors. The coronal cross-section confirms preferential water advance through the low permeability layer in the 4-minute image. Images at 90 minutes (top- and bottom-right) also confirm the inter-layer exchange observed in previous axial cross-section. Water preferentially flows from the fracture into the low permeability layer (left) and then from the low to the high permeability layer (right), while the oil phase flows in the opposite direction towards the fracture.
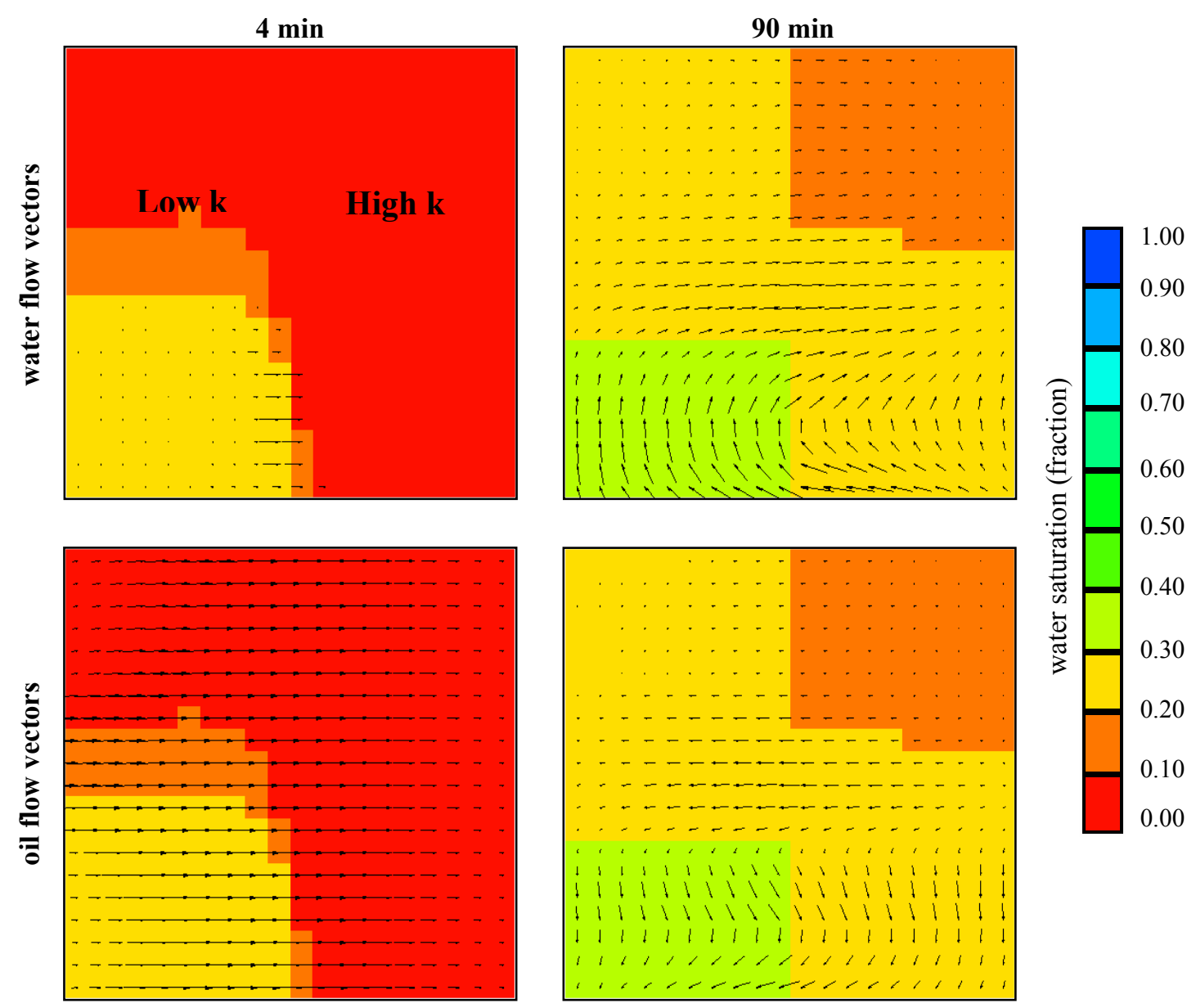

Figure 42: Water and oil flow vectors on coronal cross-section at 4 and 90 minutes, slice $y=11$. 
Figure 43 shows the $y-z$ or sagital cross-section with the corresponding water saturation changes. The sagital plane was located at $\mathrm{x}=11$, which extends through the interface between the high- and low- permeability layers (on the high permeability side) and is perpendicular to the fracture plane. The sagital cross-section reveals the tendency to equilibrate saturation distributions in the vertical direction at late times. The water saturation front flattens and rises at low rate in images at 45, 60, and 120 minutes.
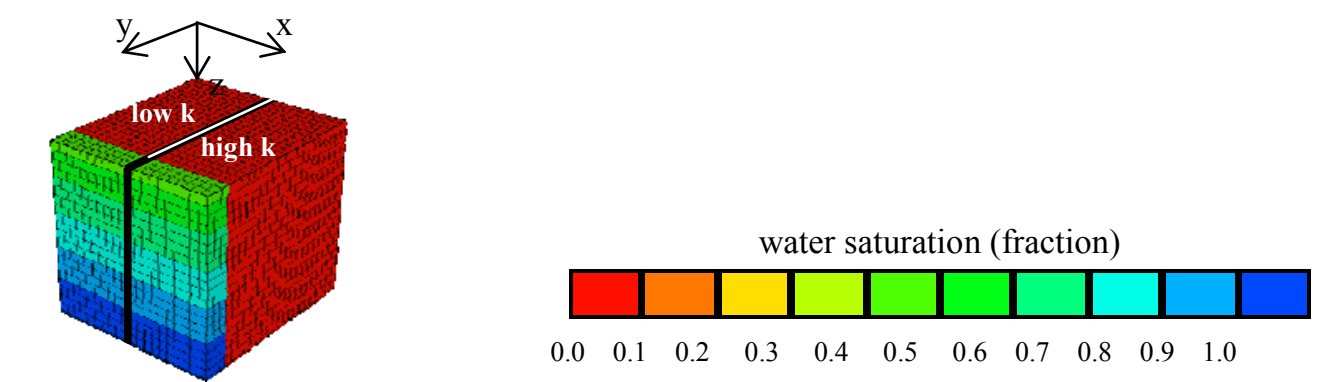

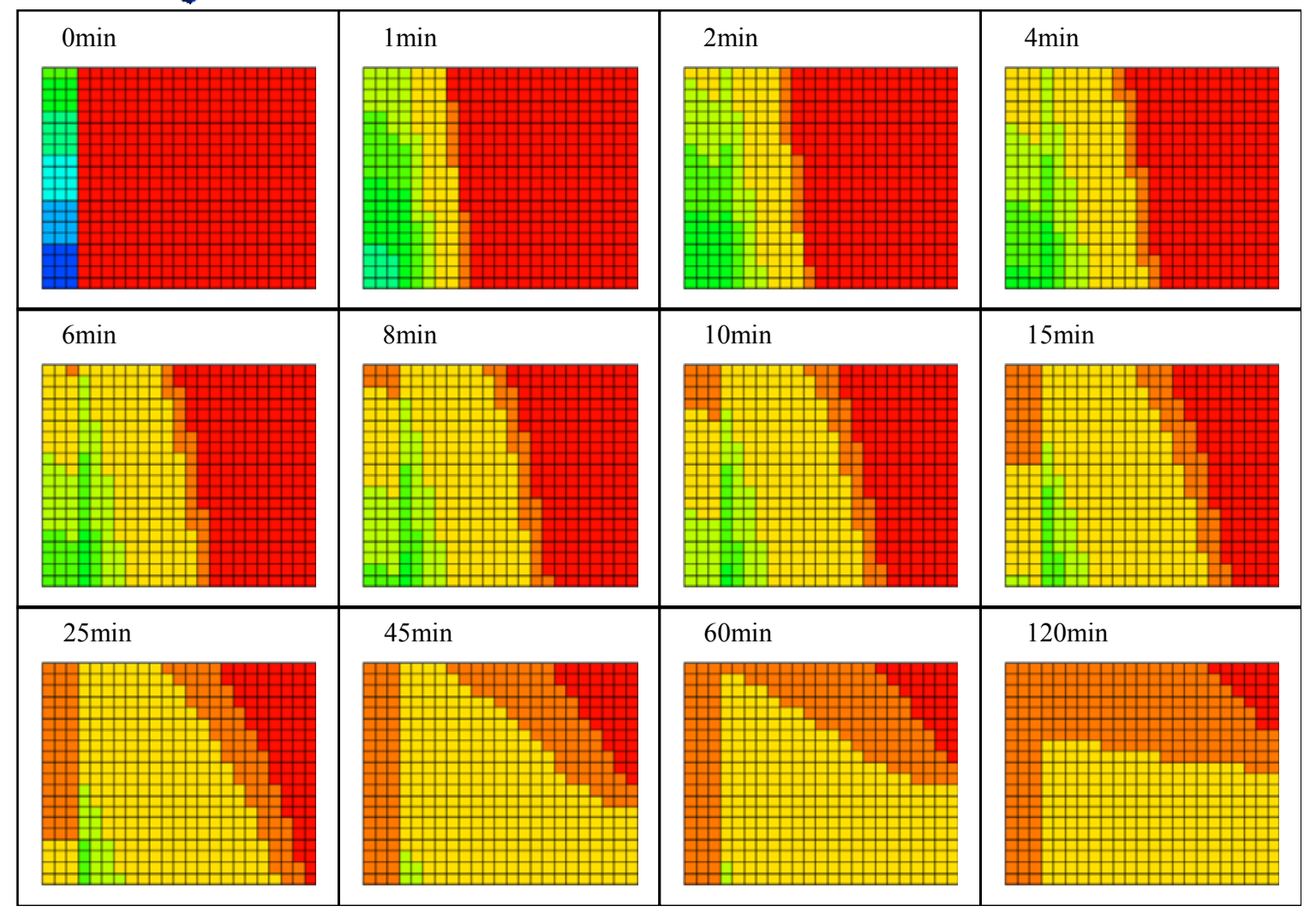

Figure 43: Water saturation changes in a sagital cross-section, slice $\mathrm{x}=11$. 
Figure 44 shows the same sagital cross-section at two different times with velocity vectors for oil and water as they exchange places in the reservoir model. The top two snapshots have water flow vectors, while the bottom two have oil flow vectors. The image at 90 minutes with water vectors (top-right) shows water migration away from the fracture and up with water flowing from the bottom of the sample and adjacent to the fracture. This observation corresponds to the experimental analysis made on the incremental water saturations obtained from digital radiography at $\Delta \mathrm{t}=15 \mathrm{hr}$ and $6 \mathrm{~min}$. At late time, the highest water saturation is found at the bottom of the sample. Capillarity drives this water towards regions with low water saturation at the top of the sample and away from the fracture. Simultaneously, oil migrates towards the bottom of the sample to replace the volume previously occupied by water. The simulated sagital cross-sections confirmed important experimental observations explaining the late-time longitudinal mechanism of water imbibition (see items 2 in Figure 1).

Experimental and simulated saturation profiles are presented in Figure 45. These profiles were computed from saturation changes in the axial cross-section of the three-dimensional model in order to compare with those obtained experimentally. Each millimeter in the "distance" axis (top-graph) corresponds to a grid block along the "y" direction on the axial cross-section. As time proceeds, regions near the fracture-matrix interface undergo drainage, while regions away from the fracture undergo imbibition. The lower portion of the curves moves from high water to low water saturation (drainage), while the upper portion of the curves moves from low water to high water saturation (imbibition). The transition zone between drainage and imbibition moves away from the fracture as the water front advances. The path of transition zones is described by the locus of intercepting points between two consecutive saturation profiles. Even though the three-dimensional simulation exercise described in this document is not a replica of the 
experimental sample or conditions, it captured the overall mechanism of capillary imbibition experienced in the laboratory.
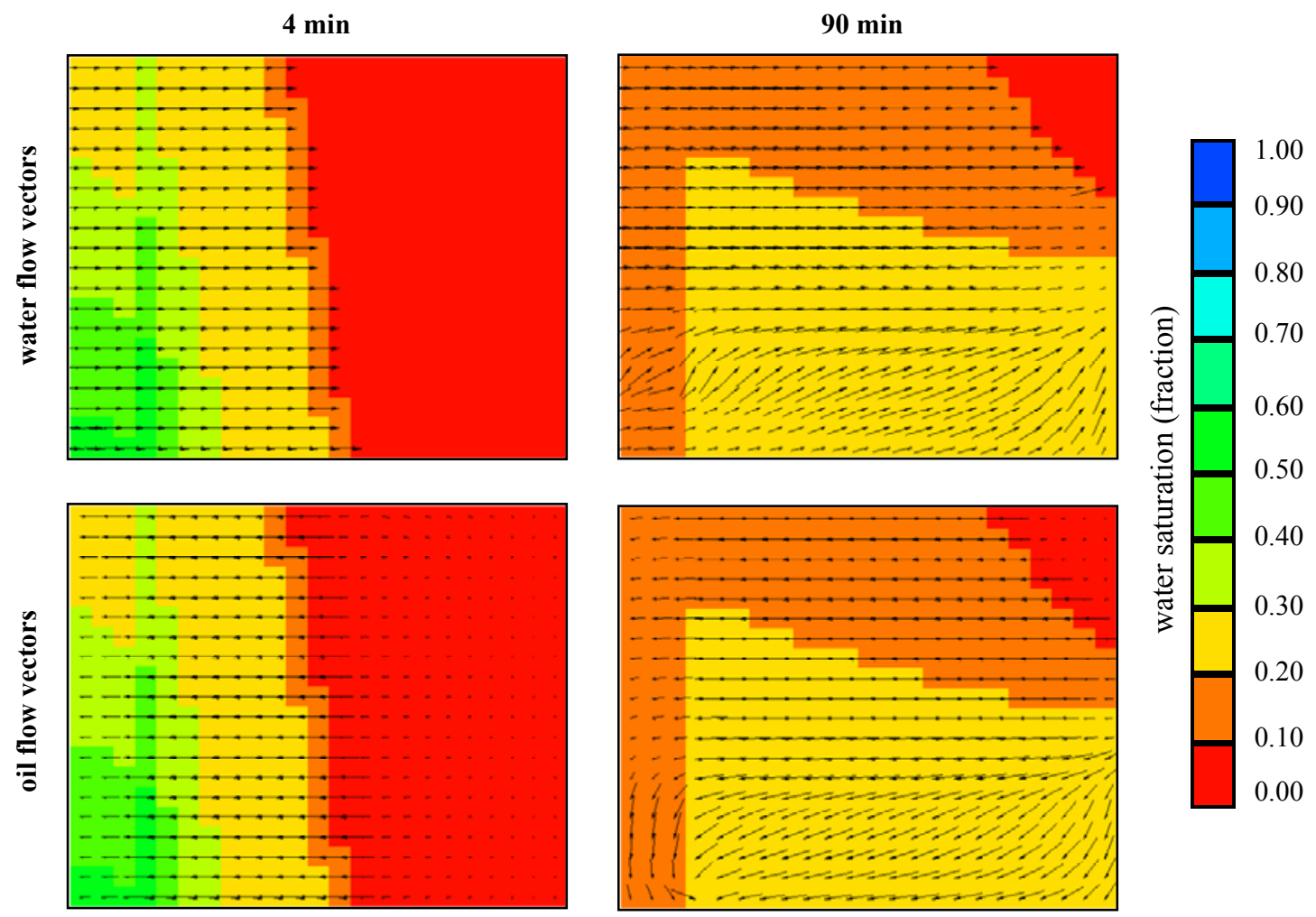

Figure 44: Water and oil flow vectors on sagital cross-section at 4 and 90 minutes, slice $x=11$. 

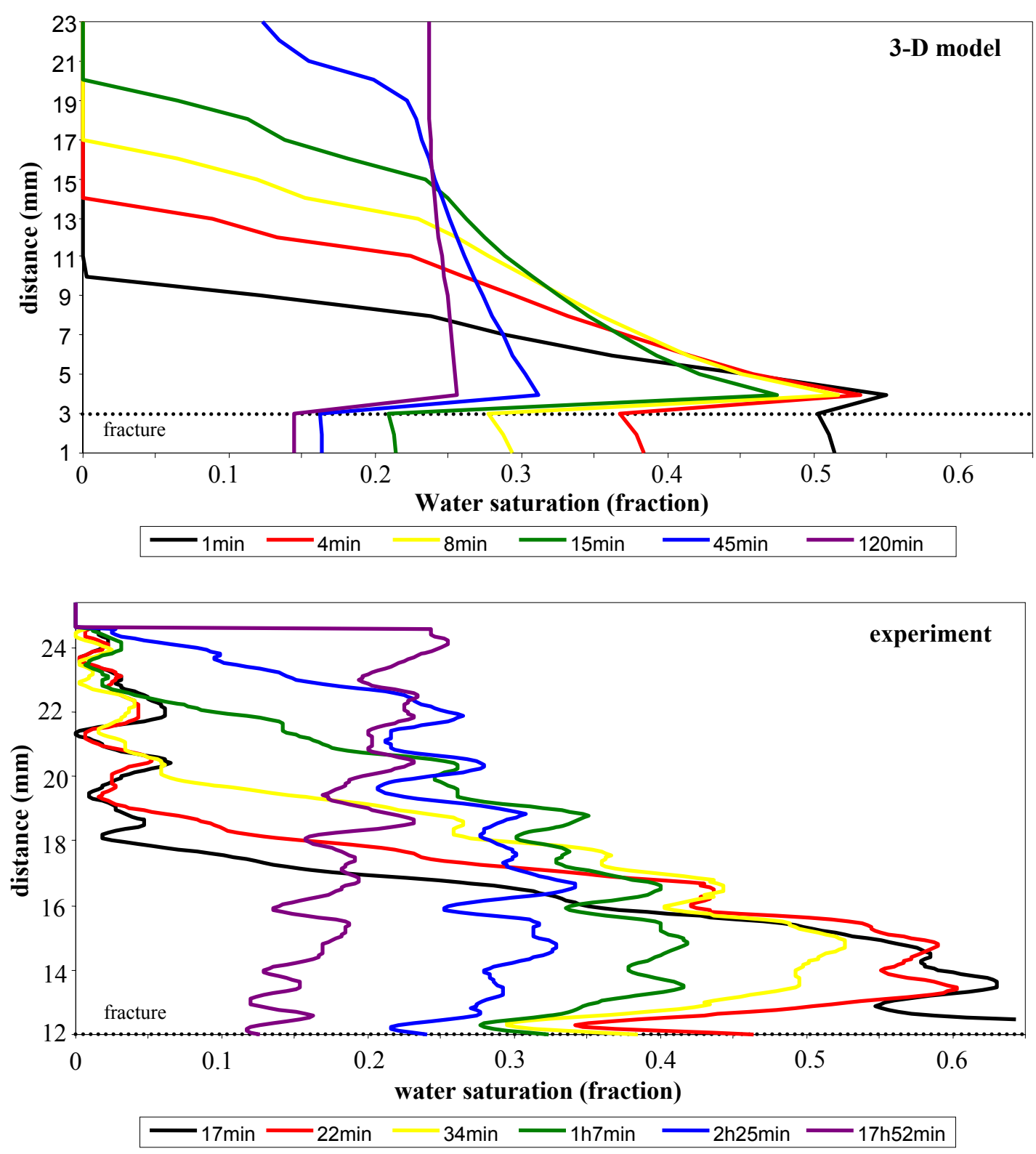

Figure 45: Qualitative comparison of cumulative water saturation profiles obtained from threedimensional simulation and the experiment.

Summary of the modeling of counter-current flow Imbibition: The present work proved the ability to detect different fluid phases coexisting in a fractured rock and to obtain detailed threedimensional fracture structures. Experimental results indicated that it is possible to characterize fracture's complex geometry and fluid phase distribution from CT data. Findings from this work 
contribute to correlation of multi-phase fluid conductivity with fracture geometry and wettability, which could significantly enhance current interpretation and modeling of capillary-driven flow in fractured systems. Using the experimental methods and modeling described in the present work, the following conclusions are made.

- A direct correlation between rock bedding porosity and local fracture apertures was confirmed. Large apertures correspond to layers of high porosity in the matrix, and vice versa. The separation of individual grains after fracturing is a possible explanation for this phenomenon. High porosity layers are typically well sorted, and have less inter-granular contact and weaker cementation than low porosity layers, thus stimulating larger apertures when fractured. This correlation plays an important role in controlling mass transport between the rock matrix and the fracture.

- There is a strong correspondence between fluid distribution and fracture geometry. The non-wetting phase tends to occupy large apertures, while the wetting phase spreads through small cavities. Due to this correspondence, it is possible to map fluid occupancy in a fracture from geometric data and wetting characteristics.

- Trapping of the non-wetting phase always occurs in local maxima with sufficient associated fracture volume which are surrounded by smaller apertures. An important consequence of fluid trapping is a high value of residual saturation of non-wetting phase, which makes the mobile region of the relative permeability curves narrower than those characteristic of matrix flow. 
- Co-current and counter-current flow mechanisms coexist during capillary imbibition of a fractured rock originally saturated with non-wetting phase. Non-destructive monitoring of fluid flow in fractured samples allowed visualization of localized co-current and counter-current flow. The presence of saturation banks during capillary imbibition suggests that there is countercurrent flow of wetting and non-wetting phases.

- Two-dimensional numerical simulation of capillary imbibition experiments demonstrated that counter-current flow prevails in the direction perpendicular to the fracture surface, while cocurrent flow dominates longitudinal imbibition, parallel to the fracture plane. Flow communication across layers also obeys the coexistence of co-current and counter-current flow.

- Accounting for capillary forces in the fracture is essential for successful modeling of fracture-matrix flow. Contact areas in the fracture provide passages under strong capillary action, which are able to drive wetting fluids into the rock matrix. Such behavior can be captured by means of fracture capillary pressures and void structure. For most part of the mobile saturation window, matrix capillary forces are higher than those in the fracture. Water available to the fracture immediately reaches for the narrowest gaps and imbibes the rock matrix near contact areas. Fracture capillary forces are especially important when the rate of injection is close to or less than the rock's capacity to transport fluids.

- The presence of bedding planes in the rock's structure determines the shape of the imbibing front. The imbibing front advances faster through low porosity/permeability layers due 
to higher capillary forces. Low porosity/permeability regions in the rock tend to coincide with the narrowest fracture gaps where water is predominantly available after water injection thus promoting advance of the imbibing front into the rock. Cross-layer fluid exchange tends to level the imbibing front. 
Shear Fractures Parallel and Perpendicular to Bedding: Two types of samples were used. The first type had cores that were cut perpendicular to bedding, and then fractured. The shear fracture is diagonal with respect to the long axis of the sample, and its orientation is arbitrary. The second type had cores that were cut parallel to the bedding plans of the rock. In this case, the fracture may have different orientations with respect to the bedding planes. Figure 46 shows the apparatus for shear fracturing. The equipment containing the rock sample (Figure 47) is subjected to simultaneous axial loading and symmetric radial confining pressure. At peak load, the stress conditions are $\sigma 1=\mathrm{P} / \mathrm{A}$ and $\sigma 3=\mathrm{p}$, where $\mathrm{P}$ is the highest load supportable parallel to the cylindrical axis, and $\mathrm{p}$ is the pressure in the radial confining medium. The confinement effect, that is, the strengthening of the rock by the application of confining pressure $p$, is realized only if the rock is enclosed in an impervious jacket.

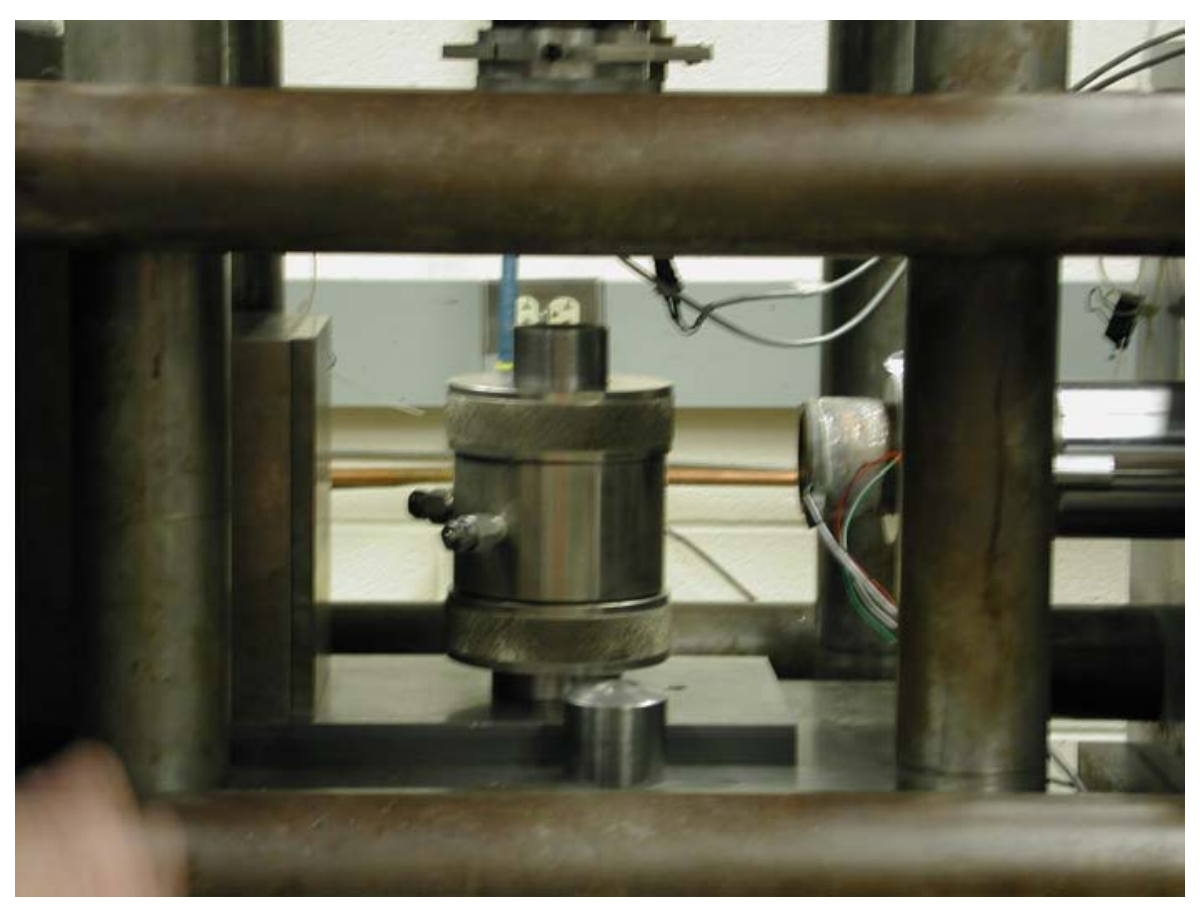

Figure46: Apparatus for fracturing in shear mode. 

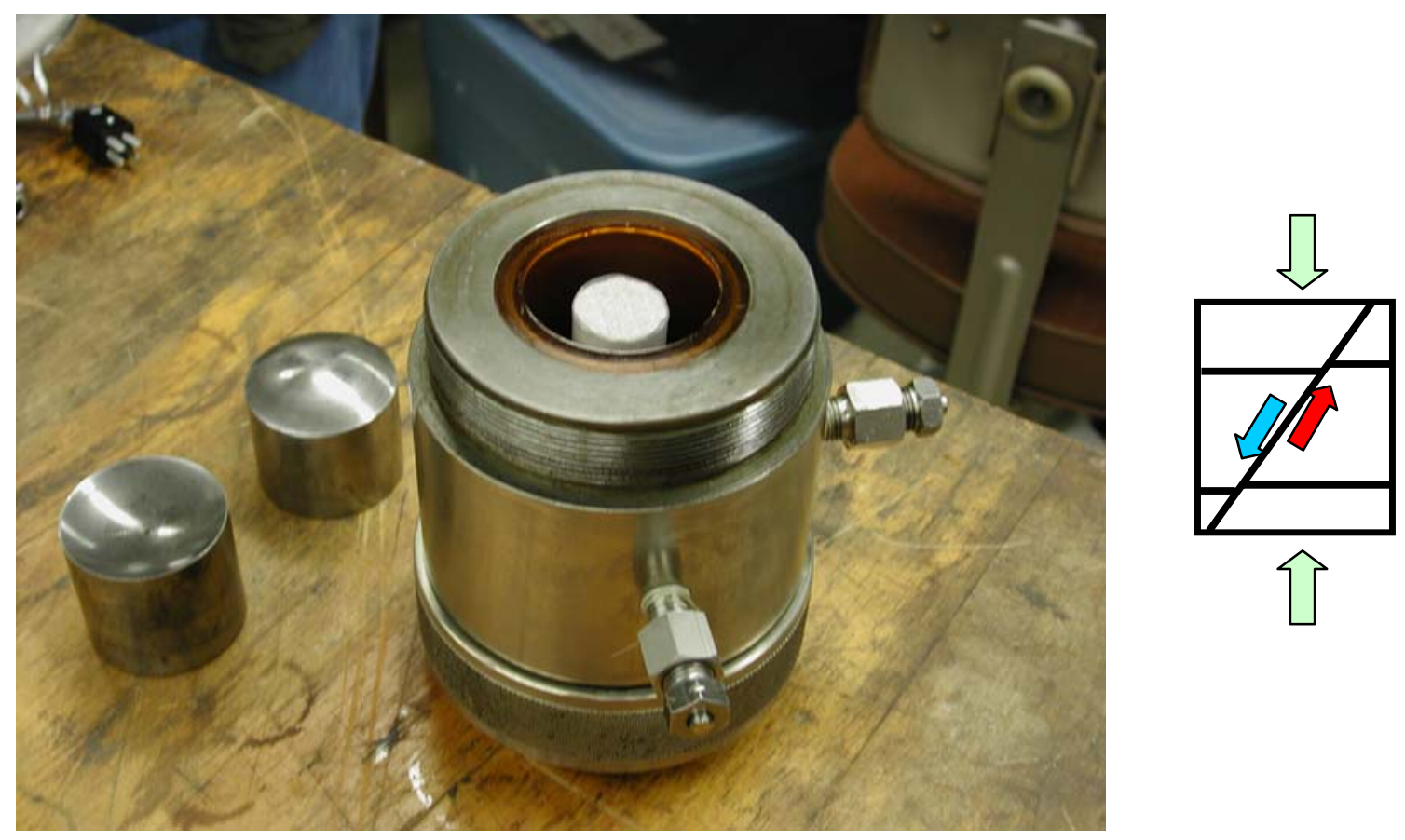

Figure 47: Packing the core holder with Aluminum oxide beads around the sample to provide the radial confining stress.

Bedding perpendicular to the main axis of the core: The absolute permeability of the four samples that were fractured in shear increased after the fracturing process. Table $\mathbf{3}$ summarizes the permeability changes after fracturing. MicroCT imaging of the samples will relate the topology of the fractures to the change in absolute permeability. The overall absolute permeability increased after fracturing. 
Table 3: Absolute permeabilities before and after fracturing

\begin{tabular}{|c|c|c|}
\hline Sample Number & \multicolumn{2}{|c|}{ Permeability (md) } \\
\hline & Before Fracture & After Fracture \\
\hline 3 & 40.1 & 68.7 \\
\hline 5 & 42.3 & 49.8 \\
\hline 7 & 43.8 & 75.3 \\
\hline 8 & 42.5 & 75.2 \\
\hline
\end{tabular}

A

chose

n

sampl

e was placed in the core flooding system for a two-phase flow experiment. The sample was scanned dry for establishing the structure of the fracture pore network. Figure $\mathbf{4 8}$ shows a series of three-dimensional renditions of the shear fracture.

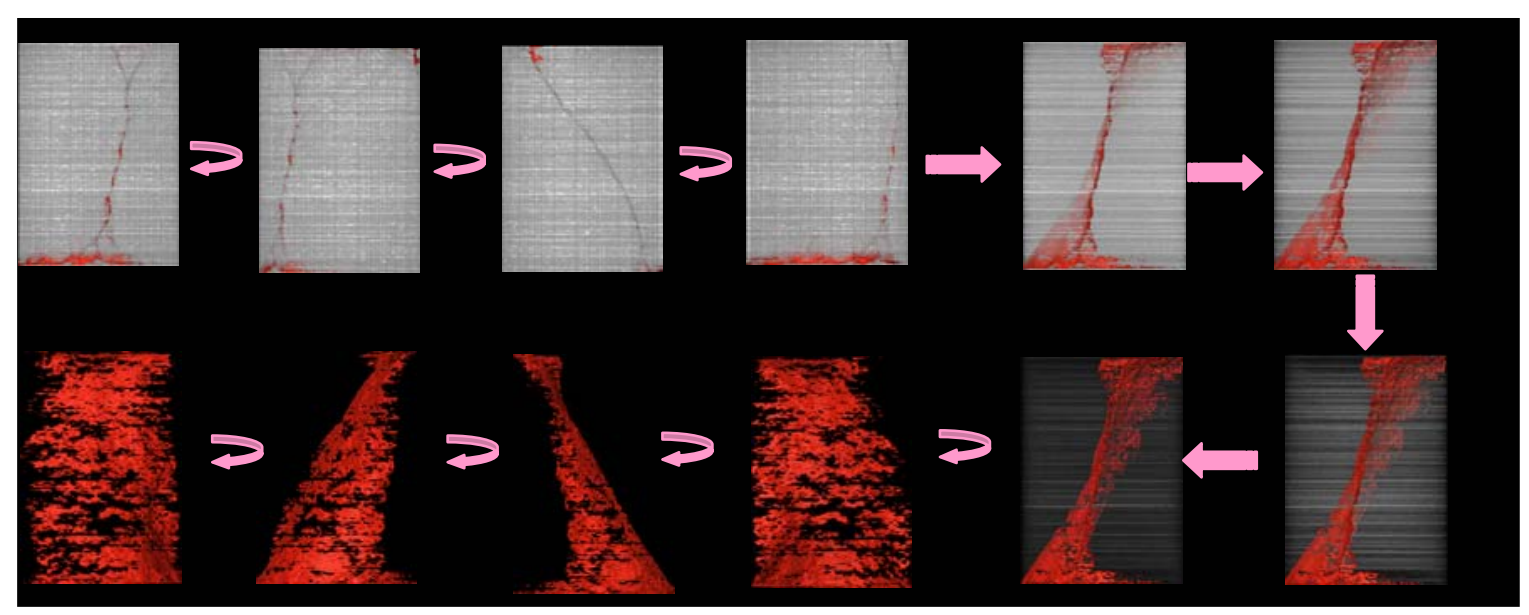

Figure 48: Three-dimensional renditions of a shear fracture in a sample cut perpendicular to bedding.

The sample was fractured while saturated with water with $2 \%$ sodium chloride, to prevent clay swelling and migration. Figure 49 shows the injection procedure followed in the experiment. At the end of each stage the sample was scanned entirely. Porosity and saturations were extracted from the CT data allowing us to understand the effect of the fracture on the displacement process. Figure $\mathbf{5 0}$ shows the overall porosity distribution along the sample. 
Figure 51 shows saturation profiles after three of the flooding stages: 1. after displacing the initial water with $\mathrm{NaI}$ tagged water. 2. after oil injection, at Swirr, and 3. after water flooding, at Sor. Figure 52 shows the net recovery after water flooding showing that high recoveries were observed in areas along the fracture that had poor fracture connectivity, thus, forcing the displacement to take place in the matrix.

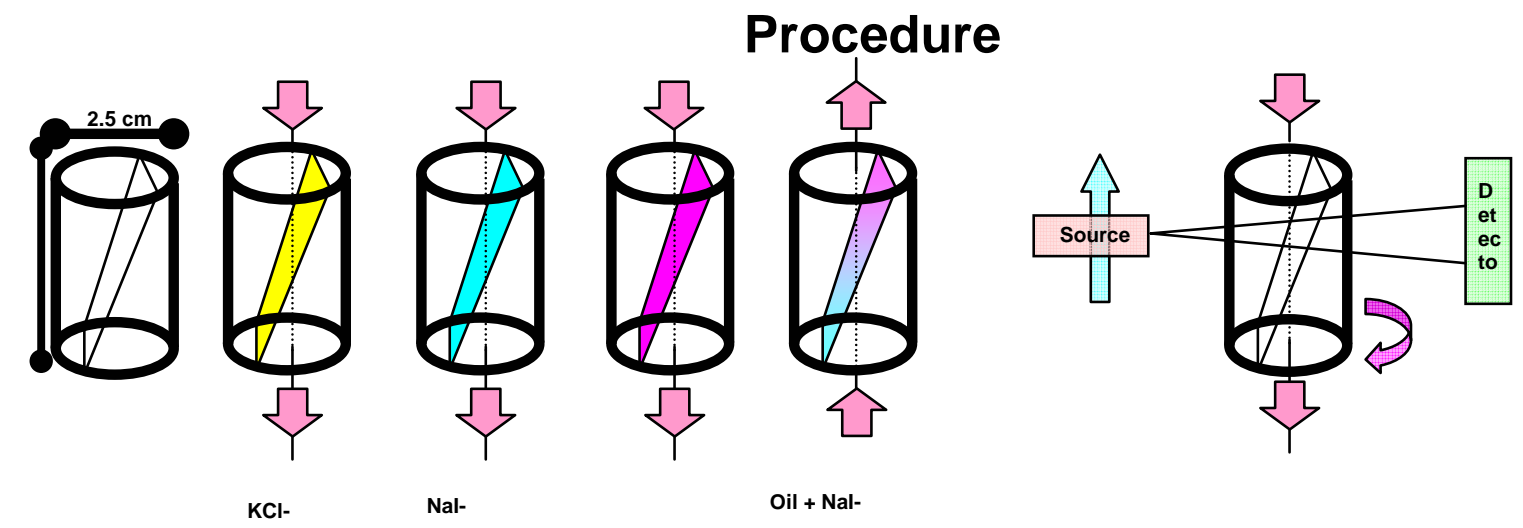

Figure 49: Experimental procedure for fluid flow experiment with shear fractures. 


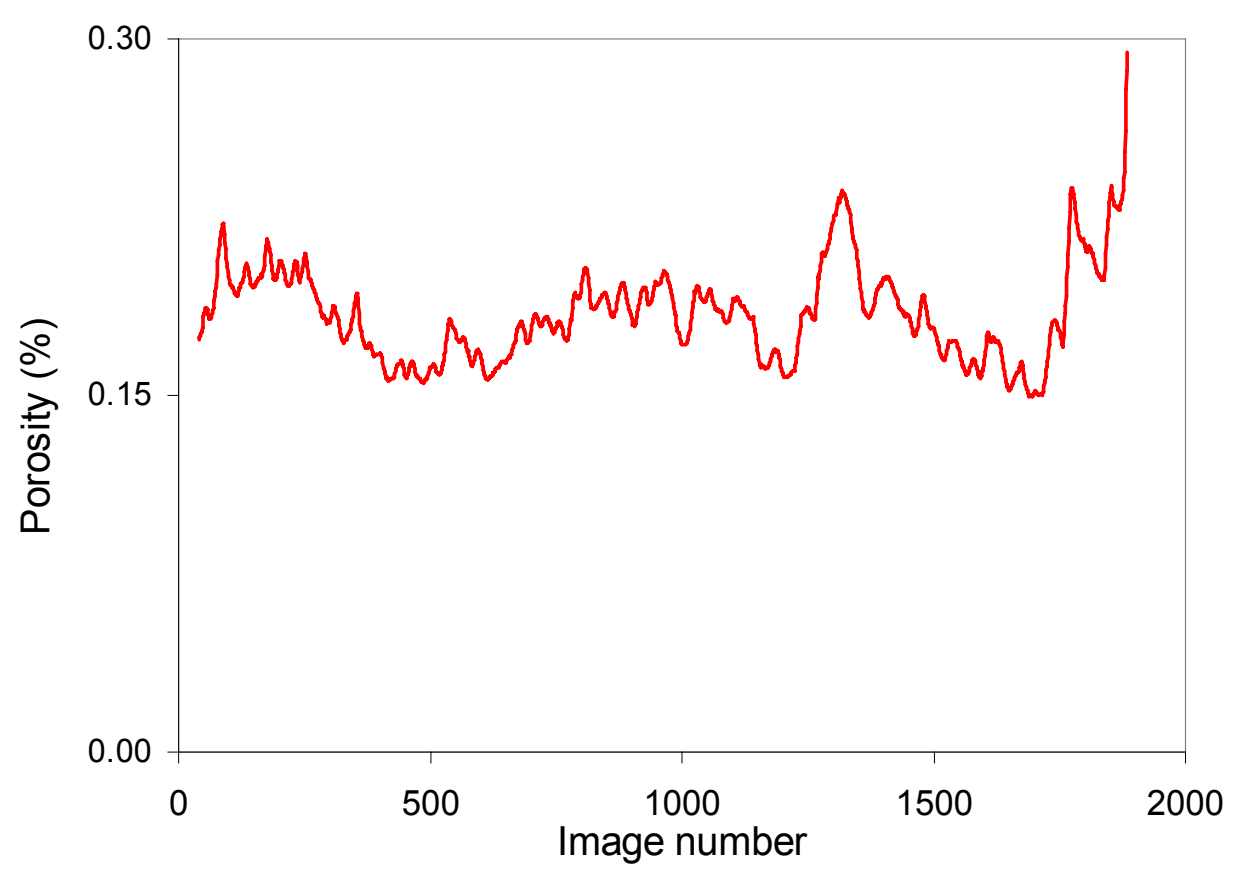

Figure 50: Porosity distribution along the sample. The inlet side is on the right. The length of the sample was $64 \mathrm{~mm}$.

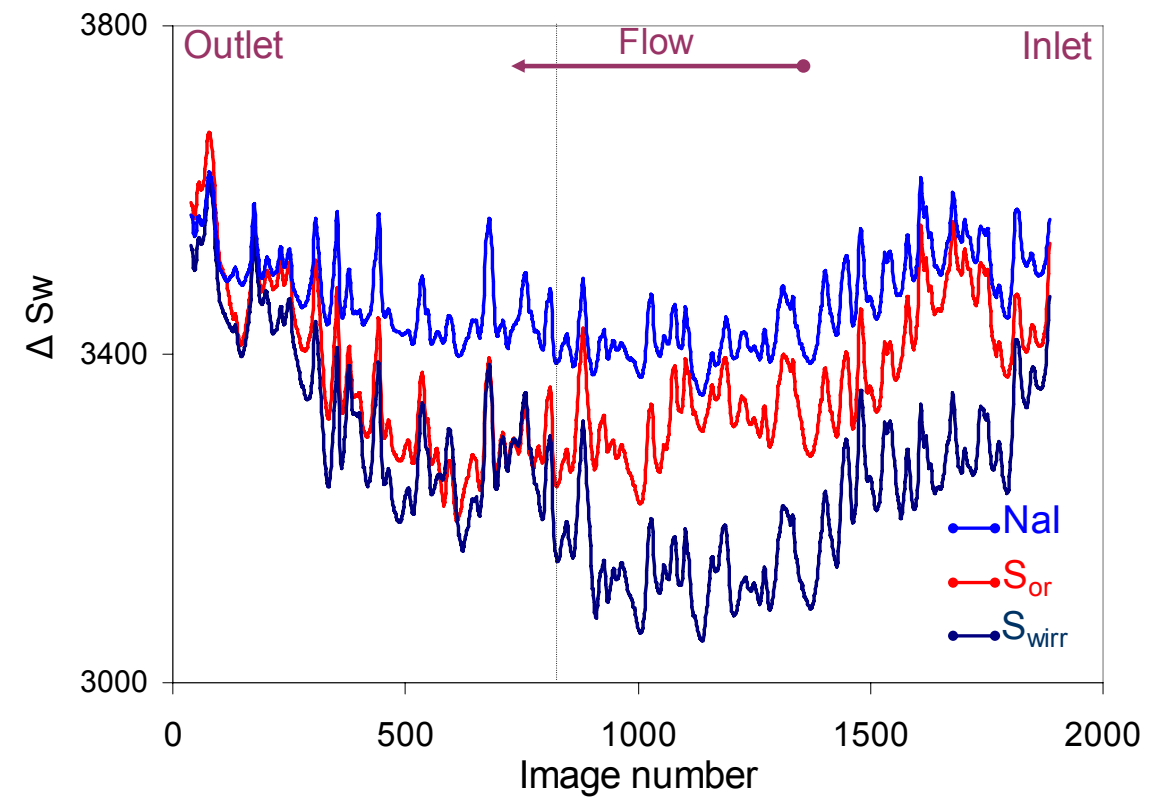

Figure 51: Saturation distribution along the sample. Blue: after replacement of water with NaItagged water. Black: after an oil flood, at residual water saturation. Red: after a water flood, at residual oil saturation. 


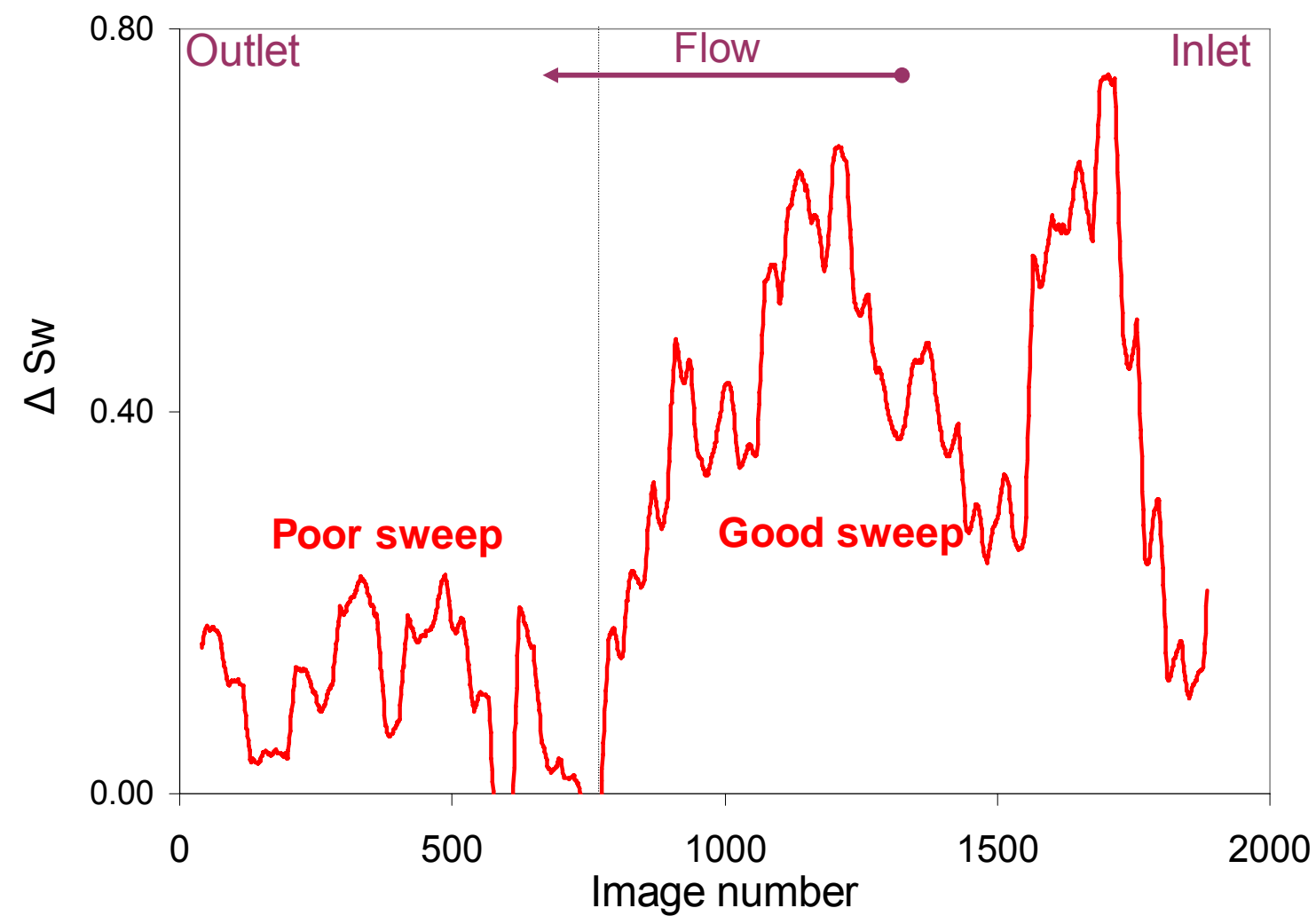

Figure 52: Net oil recovery profile along the sample showing a region of relatively good sweep that corresponds to a region with poor fracture connectivity.

Figure 53 shows three possible conditions of flow in the sample. The fracture at inlet end of the sample is less connected than the outlet end. This leads to the increased recovery shown in Figure 52. The perpendicular layers created isolated fractured shear zones that are much less connected than fractures that were created in samples that had the bedding planes parallel to the main axis of the sample. 

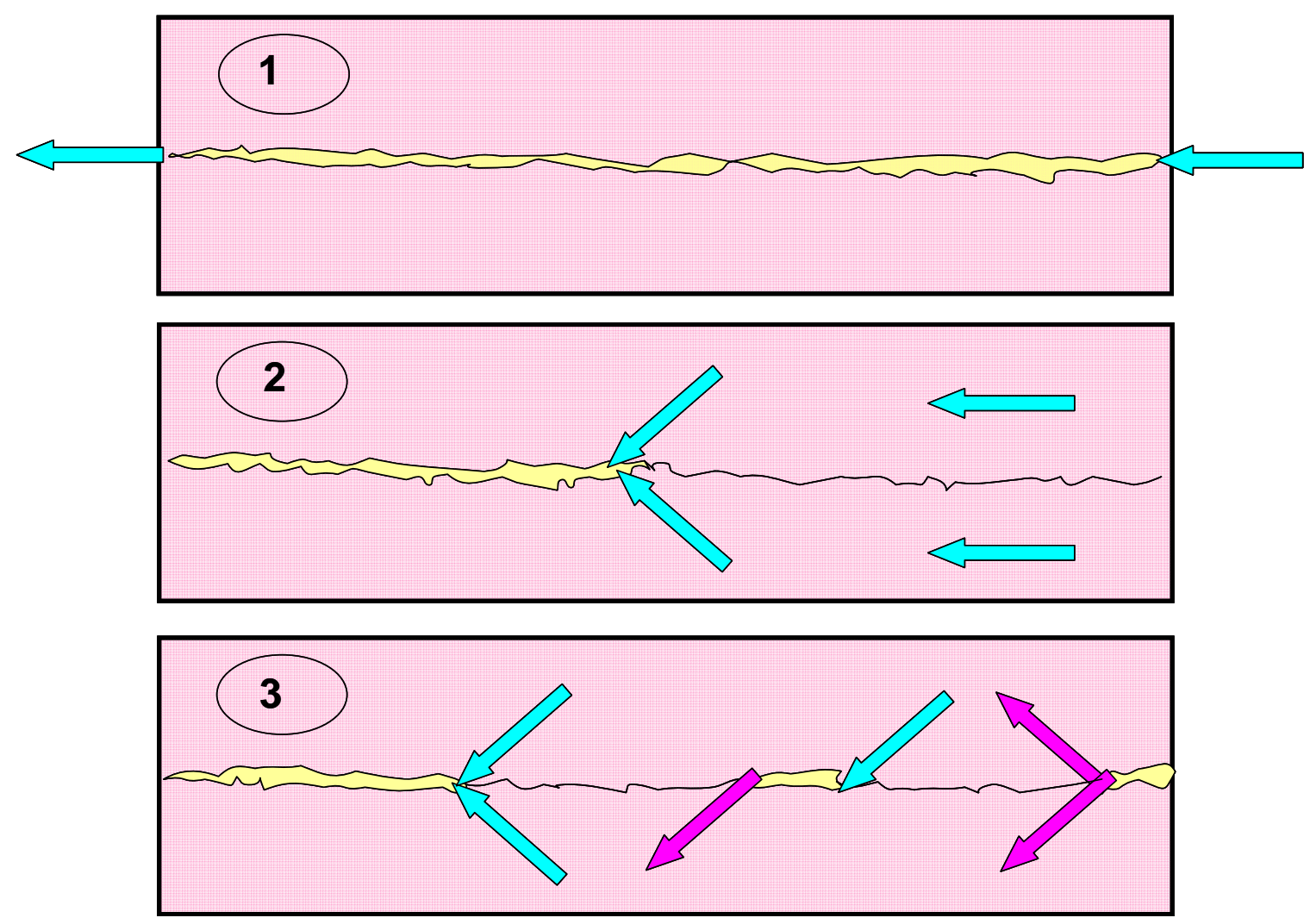

Figure 53: Three possible fracture conditions, of which the most likely one in the experiment is number three.

Bedding parallel to the main axis of the core: Several samples (one is shown in Figure 54) were fractured in shear. Two - phase flow experiments were performed in a similar manner to the ones outlined for the perpendicular bedding case, and are summarized in the Seventh SemiAnnual Report. Table 4 summarizes the reduction in permeabilities observer for the parallel bedding case. Two additional fractures were created for the perpendicular to bedding case. 


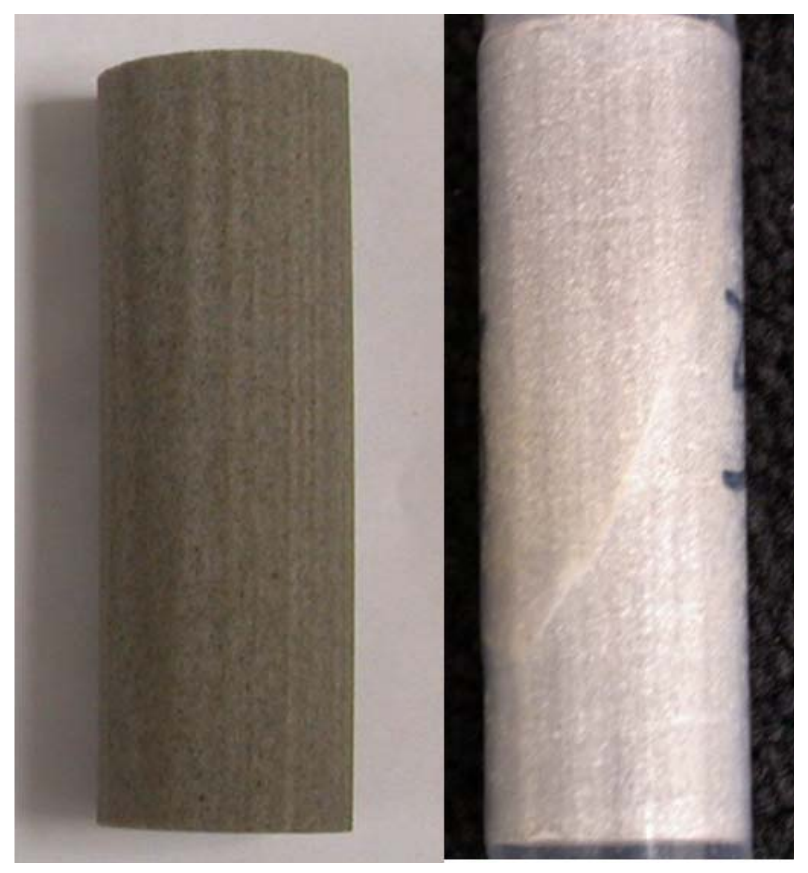

Figure 54: An example of a sample with the bedding planes parallel to the main axis of the sample, before and after fracturing.

Table 4: Air permeabilities for samples cut parallel and perpendicular to bedding planes before and after fracturing.

\begin{tabular}{|l|c|c|c|c|}
\hline \multirow{2}{*}{ Parallel } & Sample \# & \multicolumn{3}{|c|}{ Permeability, md } \\
\cline { 2 - 5 } & & pre-fractured & fractured & K change, \% \\
\cline { 2 - 5 } & 1 & 106 & 82 & -23 \\
\cline { 2 - 5 } & 2 & 86 & 78 & -10 \\
\cline { 2 - 5 } & 3 & 158 & 111 & -30 \\
\hline \hline Perpendicular & 1 & 136 & 118 & -14 \\
\cline { 2 - 5 } & 2 & 77 & 84 & +9 \\
& 2 & 67 & 77 & +15 \\
\hline
\end{tabular}


Dry Fracture Morphology: Fractures were extracted from the dry scan for the sample fractured parallel to bedding planes and perpendicular to bedding planes. The volumes of the fractures in parallel and perpendicular to bedding planes samples are $181.48 \mathrm{~mm} 3$ and $111.66 \mathrm{~mm} 3$, respectively, representing $0.75 \%$ and $0.58 \%$ of the bulk volume of the samples shear fractured parallel and perpendicular to bedding planes, respectively. The shear fracture induced parallel to the bedding planes has larger and wider distribution of the asperities than the fracture induced perpendicular to the bedding planes. Figure 55 shows partitioned maps of the fractures induced parallel and perpendicular to bedding planes extracted from dry scans.

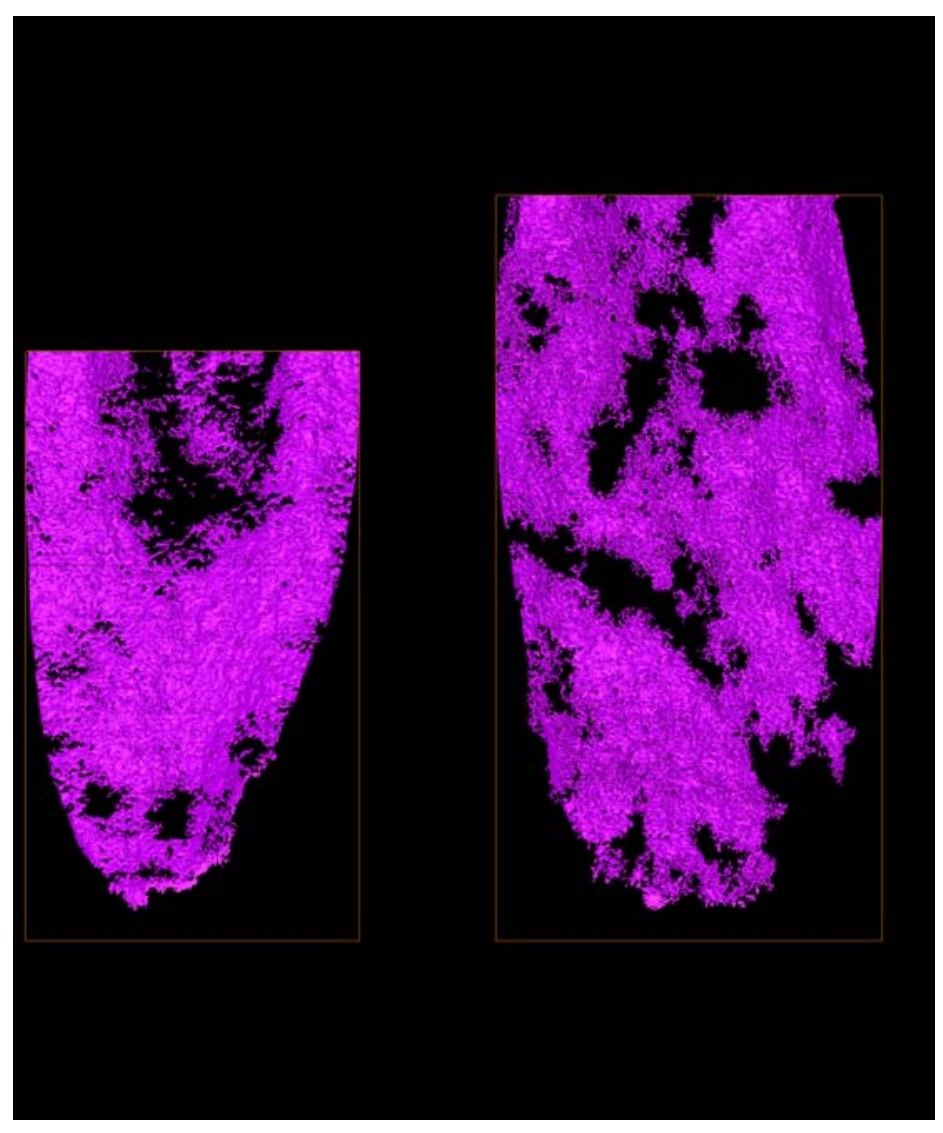

Figure 55: Binary maps of the fractures created in perpendicular and parallel to bedding planes samples. Left: Perpendicular to bedding. Right: Parallel to bedding. 
The reduction in permeability in the parallel to bedding planes cores could be explained by the dislocation of the layers. The fracture was displaced be $500 \mathrm{~m}$. Because the fracture was introduced parallel to the bedding planes, this displacement resulted in dislocation of the layers where the low density layer faced a high density layer and vise versa as shown in Figure $\mathbf{5 6 .}$ Layers dislocation forces the fluid to change its path, and as a result change the permeability. Another possible reason for the reduction of permeability could be the gouge formation after the introducing the fracture. The permeability of these regions in the direction of the flow is very low, thus, reducing the overall permeability. Gouge formation can not be shown directly in the fracture map extracted from the dry scan, but it will be discussed in coming sections.

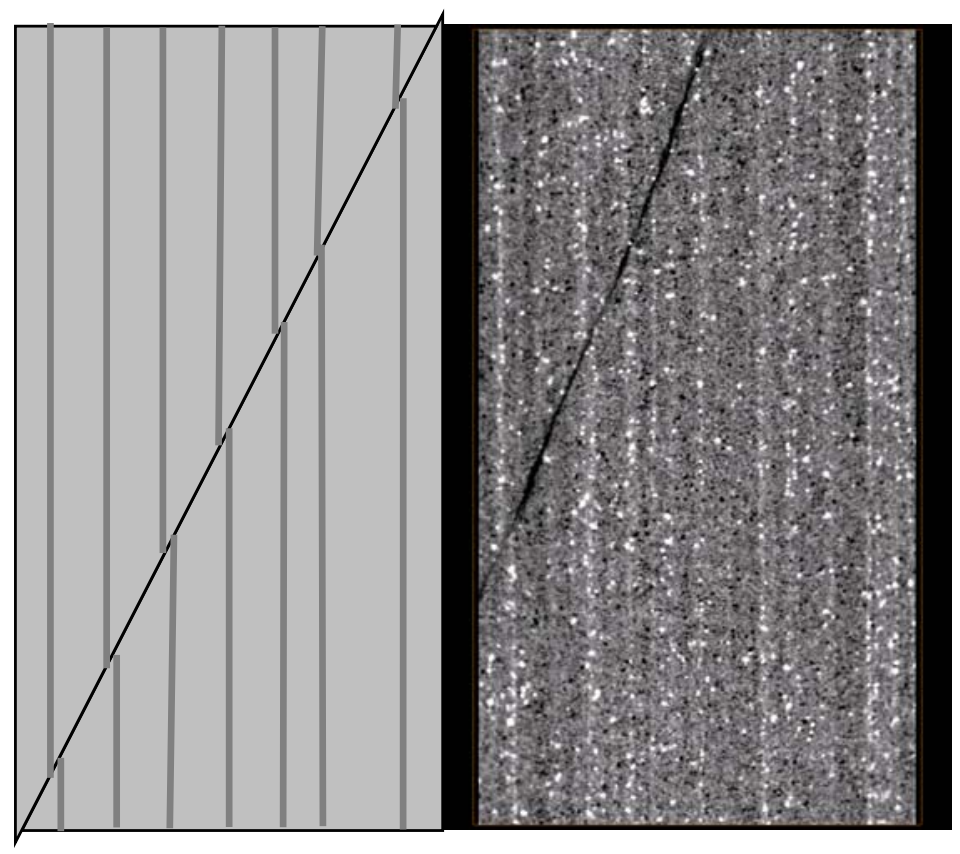

Figure 56: Schematic and actual layers dislocation after fracturing.

Two phase flow experiments that are summarized in the Seventh Semi-Annual Report yielded a final oil recovery at the end of a water flood shown in Figure 57. About 15\% of the oil was produced from the core sample after water flooding. Pressure data were collected at Swirr and 
Sor condition. Relative permeabilities to kerosene at Swirr and to water at Sor are 0.4 and 0.1 , respectively. These relative permeabilities were compared to permeability values calculated for non-fractured samples at Swirr and sor conditions. Water saturation at Swirr and Sor values for non-fractured sample are $47 \%$ and $80 \%$, respectively. Relative permeabilities to kerosene at Swirr and to water at Sor are 0.89 and .09 , respectively. Saturation values for fractured and nonfractured samples show that there is not much difference in the value of Swirr. There is a large difference in Sor values indicating that oil recovery from the non-fractured sample is larger than that produced form fractured sample. Table 4 summarizes relative permeability calculations for fractured and non-fractured samples.

The high-resolution CT images allowed us to extract the distribution of the oil and water in the fracture at all stages of the experiment. Figure 58 shows the water saturation map at residual water condition, and Figure 59 shows the oil saturation map at residual water saturation. Figure 60 shows the two saturations together. 


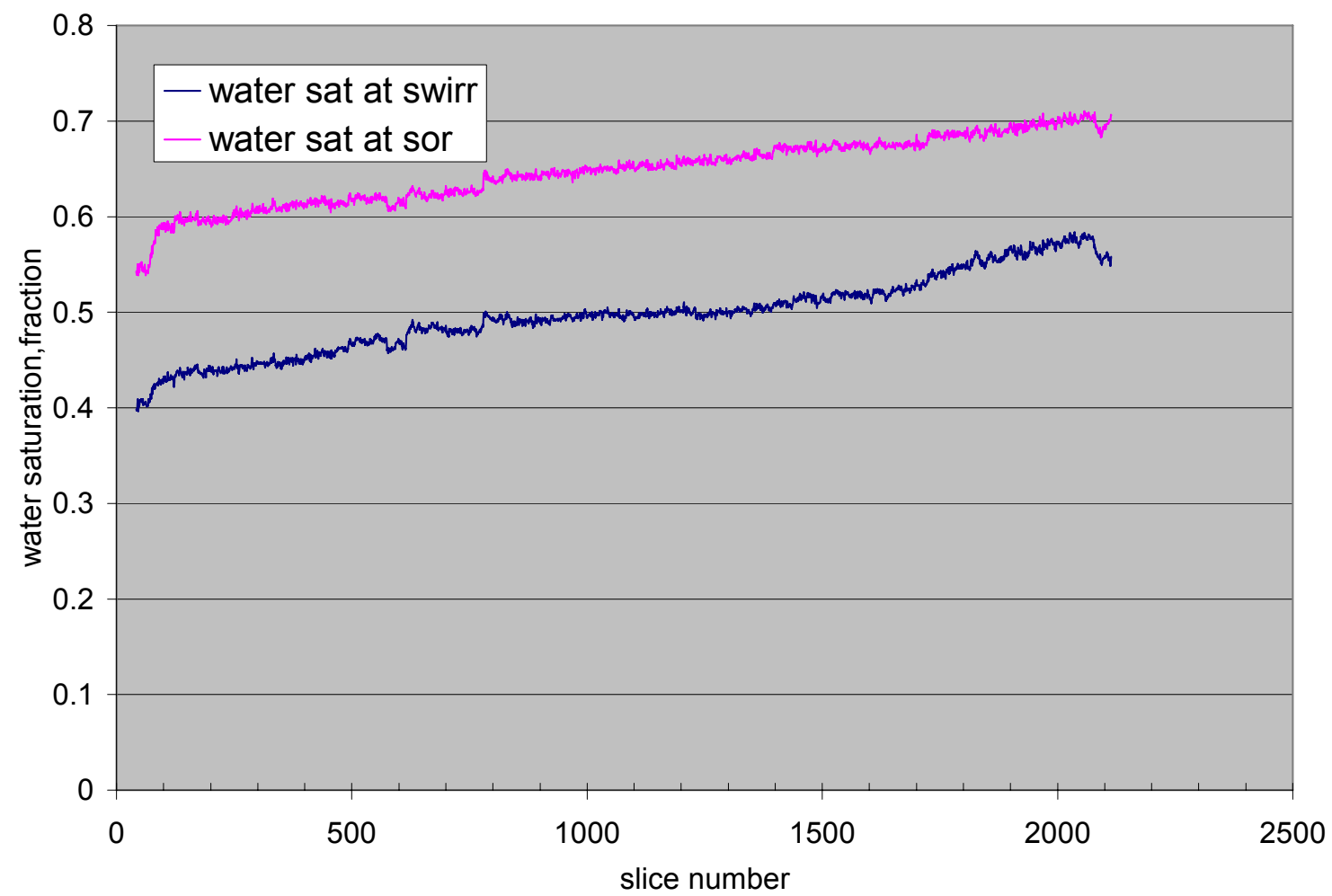

Figure 57: Average water saturation for each slice at Swirr and Sor conditions.

Table 5: Relative permeabilities at $S_{\text {wirr }}$ and $S_{\text {or }}$ for fractured and non-fractured samples.

\begin{tabular}{|l|r|r|r|r|}
\hline & $\mathrm{S}_{\mathrm{or}}, \%$ & $\mathrm{~S}_{\text {wirr }} \%$ & $\mathrm{k}_{\text {ro }} @ \mathrm{~S}_{\text {wirr }}$ & krw@S \\
\hline fracture & 35.6 & 49.1 & 0.40 & 0.10 \\
\hline non-fracture & 20.0 & 47.0 & 0.89 & 0.09 \\
\hline
\end{tabular}




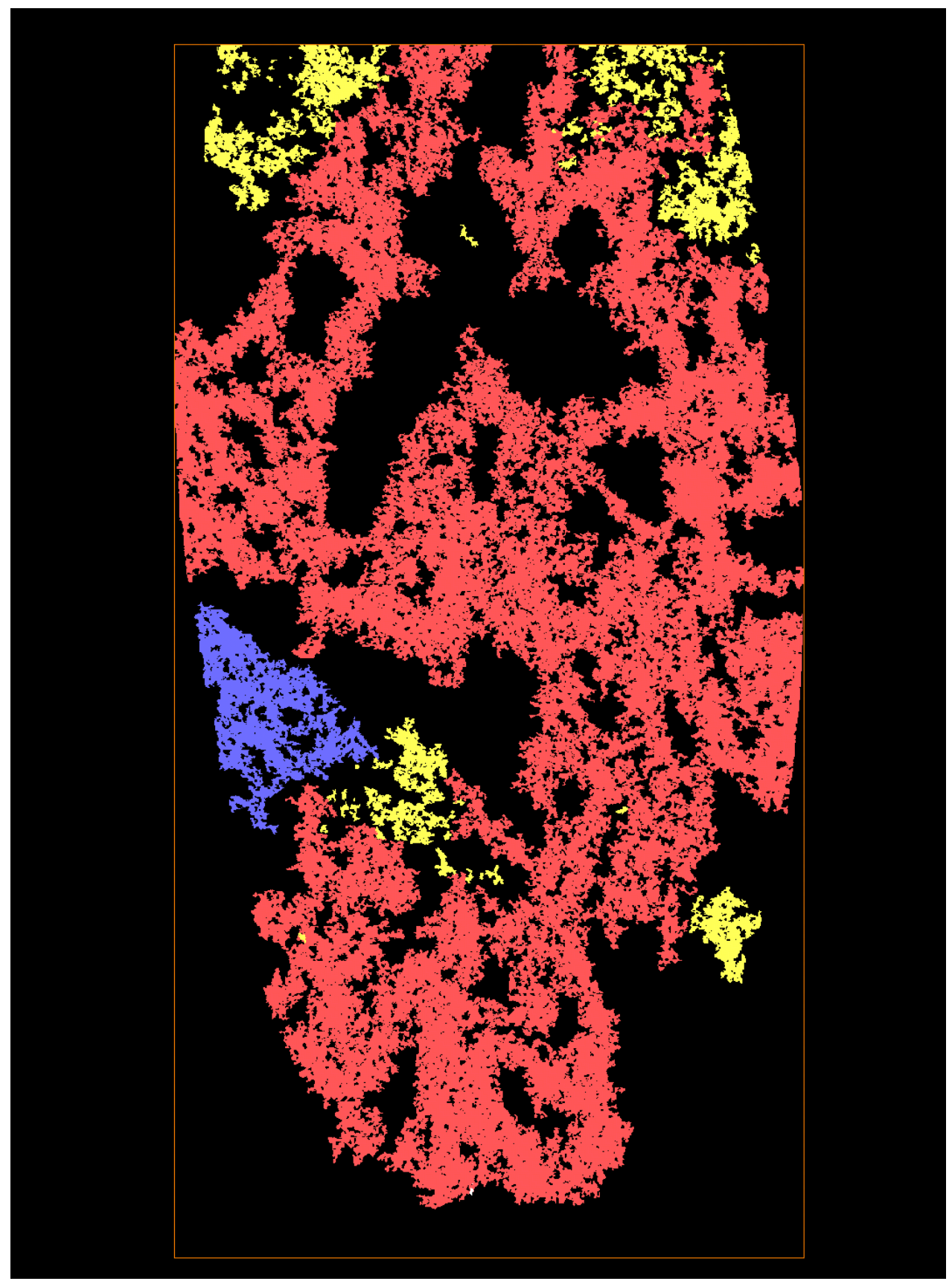

Figure 58: Water saturation inside the fracture at Swirr condition. 


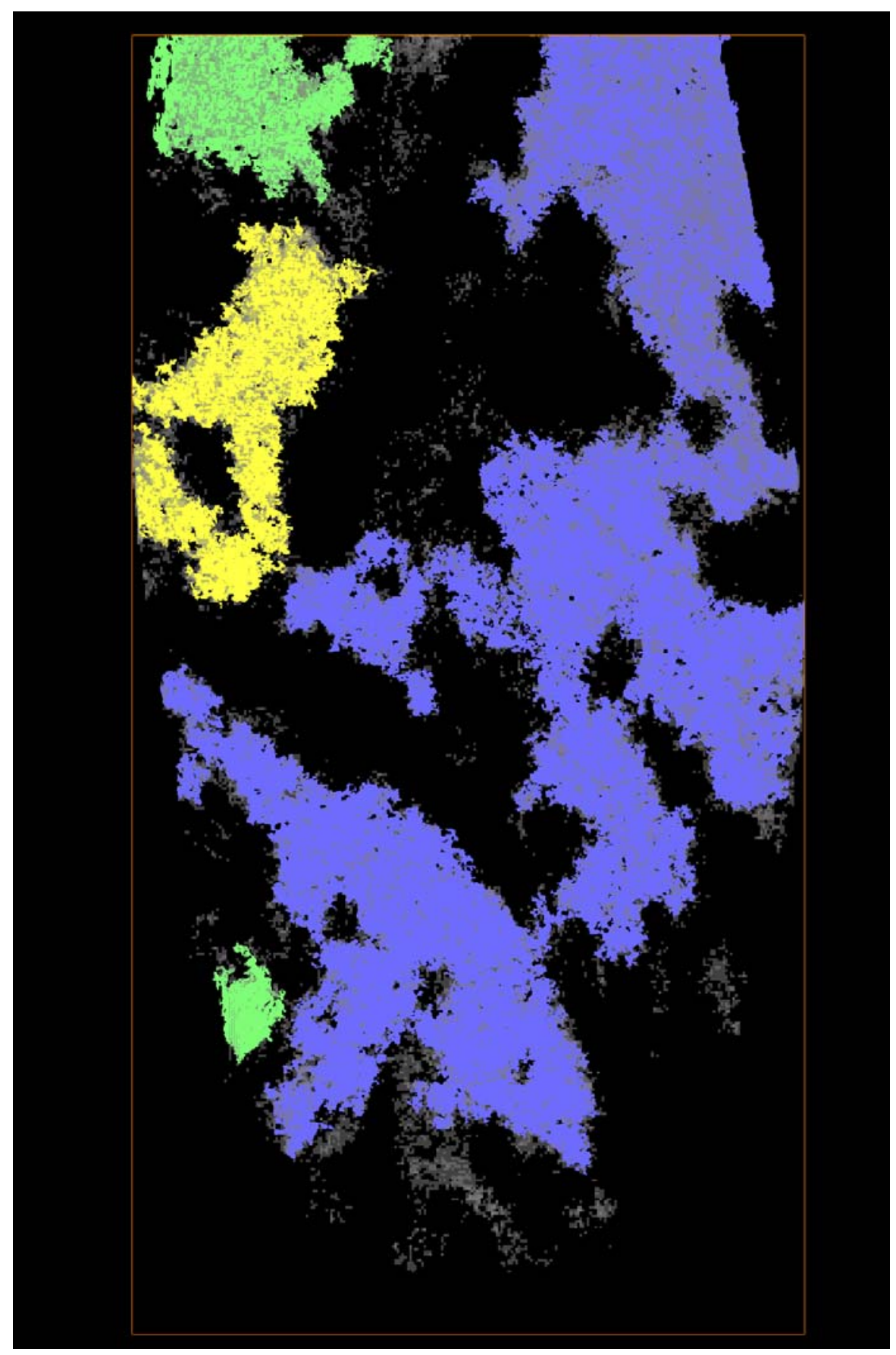

Figure 59: Oil saturation inside the fracture at Swirr condition. Dark gray is the fracture. 


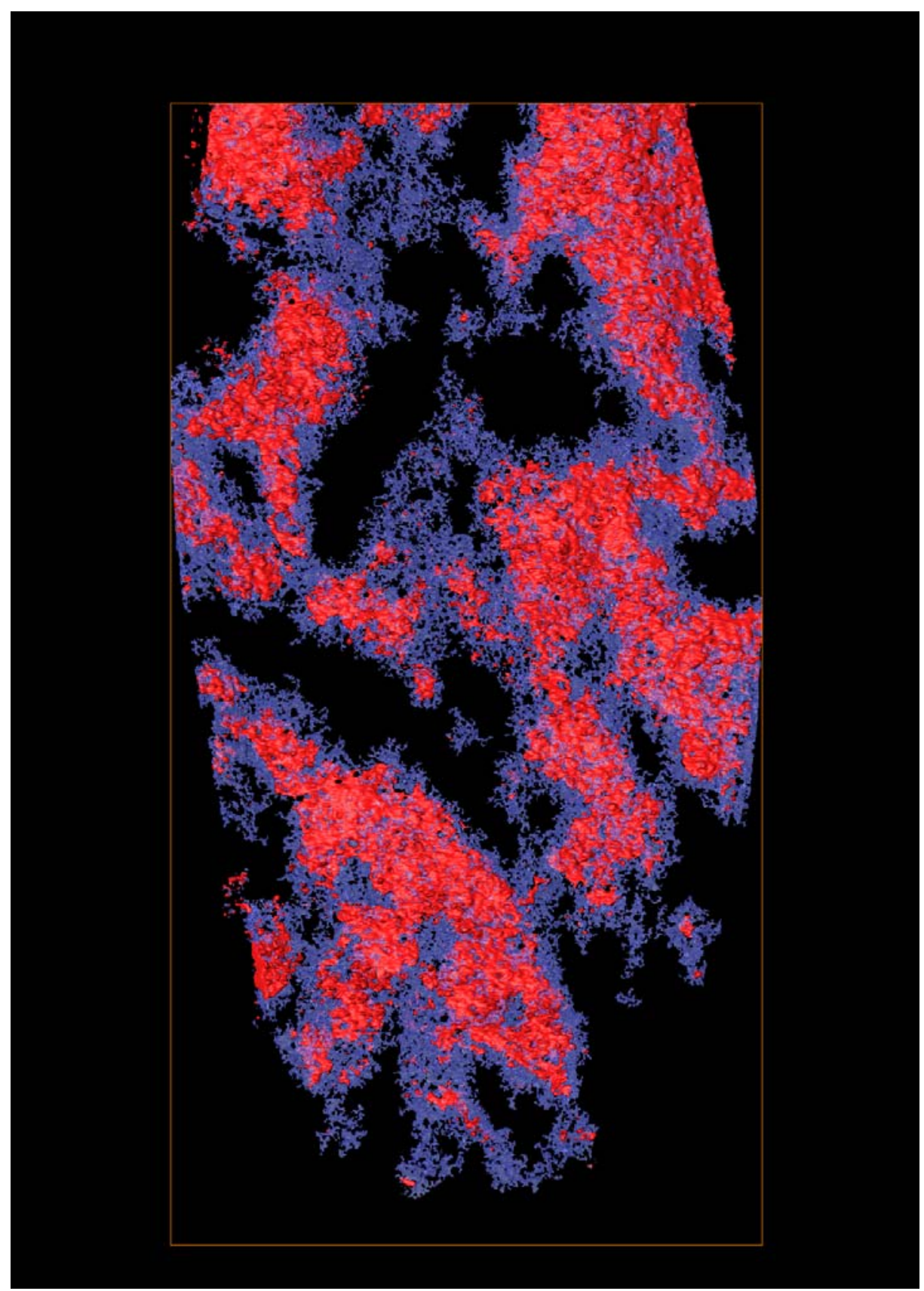

Figure 60: Both oil and water saturation at Swirr condition. Blue is water and red is oil. 
Tables 6 and 7 show the fluid occupancy fractions at the end of the oil flood and the water flood. These tables show that about $19 \%$ of the oil was produced from the fracture after water flooding. Even though the fracture was working as a conduit to the fluid, but still the Sor value is high. This is because most of the fracture's wall is water wet and most of the oil located at the center of the fracture in large aperture areas, so when flooding with water, the water prefers to flow mainly near the fracture wall and leaves the center of the fracture oil saturated.

Table 6: Water and Oil saturation at $S_{\text {or }}$ condition.

\begin{tabular}{|c|r|c|c|}
\hline Material & Count & Volume, $\mathrm{mm}^{3}$ & Percentage \\
\hline Fracture & ----------- & 181.44 & --------- \\
\hline Oil at sor & 2698137 & 81.71 & 45.04 \\
\hline Water at sor & 3292910 & 99.73 & 54.96 \\
\hline
\end{tabular}

Table 7: Water and Oil saturation inside the fracture at $S_{\text {wirr }}$ condition.

\begin{tabular}{|c|c|c|c|}
\hline Material & Count & Volume, $\mathrm{mm}^{3}$ & Percentage \\
\hline Fracture & ----------- & 181.48 & -------- \\
\hline oil_at_swirr & 3809310 & 115.37 & 63.57 \\
\hline water_at_swirr & 2183096 & 66.12 & 36.43 \\
\hline
\end{tabular}

Summary of shear fracture experiments: This exploratory study addresses the effects an artificial shear fracture created parallel to the bedding planes on absolute permeability and fluid 
distribution in two-phase flow experiment. Absolute permeabilities were measured before and after fracturing for shear fractures created parallel to bedding planes and perpendicular to bedding planes. Absolute permeability reduces $10 \%$ to $30 \%$ after fracturing induced parallel to bedding planes, while increases $9 \%$ to $17 \%$ after fracture induced perpendicular to bedding planes. Morphology of the fractures in both cases were compared and found that the shear fracture induced parallel to bedding planes has more asperities which distributed more than the asperities in the shear fracture induced perpendicular to bedding planes. Water and oil distributions were measured in the shear fracture induced parallel to bedding planes at Swirr and Sor saturation conditions. The value of Sor in the fracture is higher than in the matrix. Values of Swirr and Sor in a non-fractured sample were higher than the values in the fractured sample.

To better understand the effect of shear fractures on the multi-phase flow other parameters need to be evaluated, effects of confining pressure, effects of temperature, effects of wettability, effects of the fluids used in the experiments, types of porous media, displacements of the shear fracture, and core rock heterogeneities.

Effects of Confining Stress on Aperture Structure and Two-Phase Flow: The preliminary work on this task has been reported in the seven Semi-Annual reports. The focus of this section is on the progress made during the last half year of work of this project. 
Fracture Characterization: The scanned Berea sandstone cores had a diameter 25.0 $\mathrm{mm}$ and lengths between $64 \mathrm{~mm}$ and $70 \mathrm{~mm}$. The core shown in Figure 61 was loaded into a core holder in a flexible sleeve with opposing shims of $0.4 \mathrm{~mm}$, creating an axial shift. Then, the sample was placed into the scanner and 2250 slices were acquired covering the entire length of the sample. One of these slices is shown in Figure 61a. Once the slices were obtained they were rotated with an angle between 15 and 17 degrees. This rotation allows a better manipulation of the data in the x-y plane, Figure $\mathbf{6 1 b}$. The slices were rotated during the sinogram reconstruction process, thus avoiding the introduction of any artifacts. After rotating the slices they were cut to eliminate the area beyond the edges of the core. The center of each image was identified and then, using the known radius the slices were cut, and all external pixels were arbitrarily changed to a fixed value (mask), Figure 61c. A final step consists in reducing the cut and masked images to a rectangular shape that contains only the fracture, eliminating most of the matrix region.

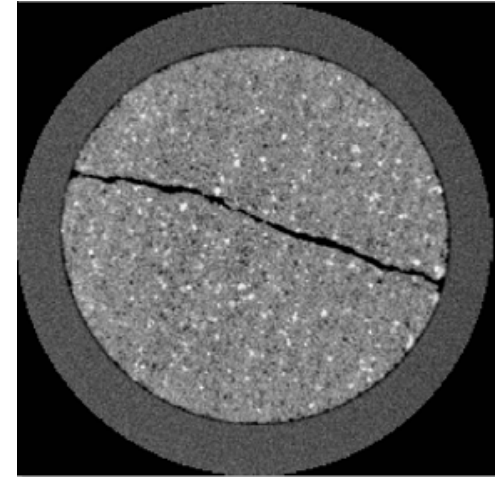

a

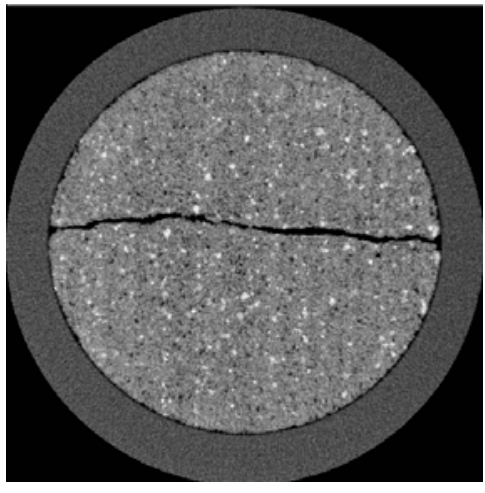

b

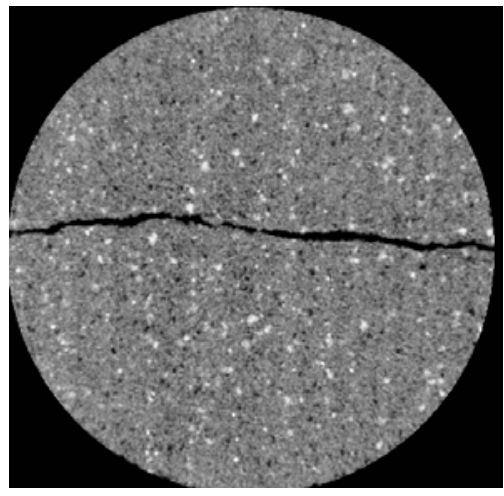

c

Figure 61: A single CT slice at various stages of processing. a: original. b: rotated. c: cut and masked. 
The cropping process reduces the size of the images and makes all future manipulations more efficient than if the original size is used. For this study an original matrix of $1024 \times 1024 \times 410$ (core BS1) or $1024 \times 1024 \times 2250$ (core BS2) was reduced to a size of $800 \times 100 \times 410$ and $850 \times 120 \times 2203$ respectively. Figure 62 shows a photograph of the one inch core before scanning (left). The final rectangle (800x100) in photograph 5.7 (right) contains the extracted fracture.

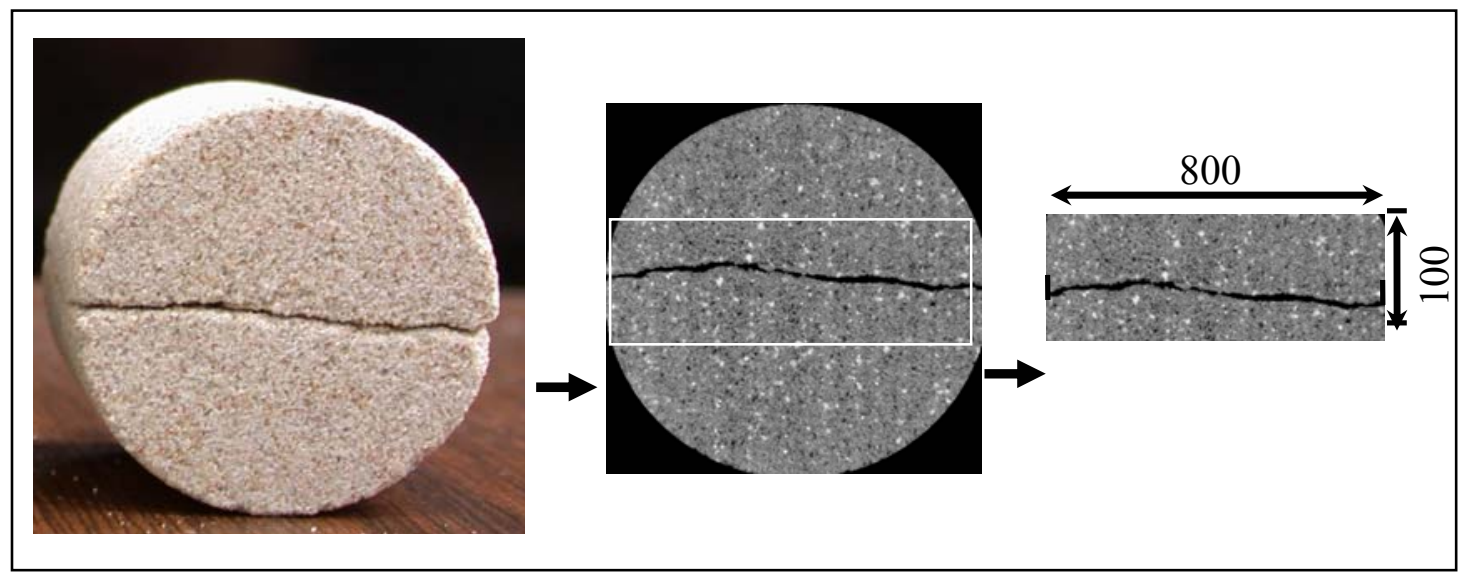

Figure 62: Original core before and after scanning and extracting a region that contains the fracture.

Figure 63 shows 6 stages of a preliminary experiment at a fixed location in the core. From left to right the confining pressure increases. The applied confining pressures are, $100,500,1000,1500,2000$ and 2500 psig, reducing the fracture aperture. The white circles in Figure 5.8 highlight the formation of a new asperity (fracture aperture closure) during the increase in confining pressure from 100 to 2500 psig. This set of data (1024x1024) is the base for extracting the fracture in order to quantify the confining pressure stress effect in three dimensions. 
a: 100 psig

b: 500 psig

c: 1000
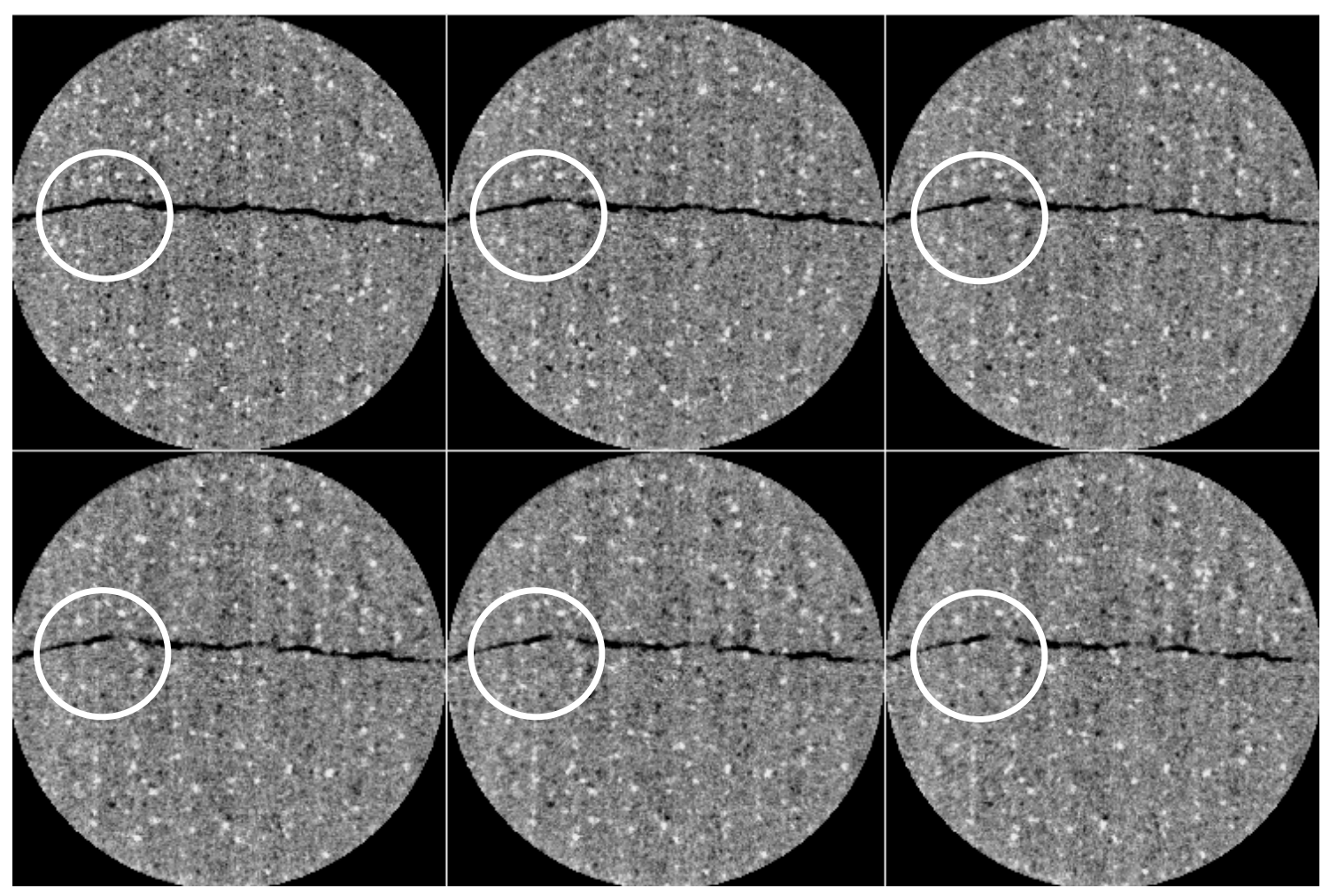

d: 1500 psig

f: 2000 psig

d: 2500 psig

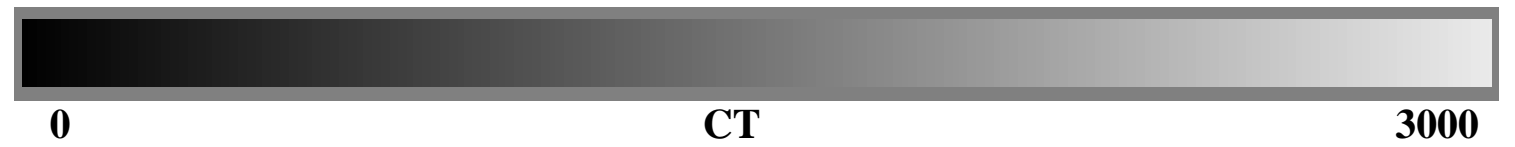

Figure 63: CT image at the same location for increasing confining pressure Slice 140.

Figure 64 shows CT profiles for different confining pressure values at the same location in the CT slice at the top. The CT profiles highlight an axial displacement of the core as a consequence of the expansion of the aluminum core holder. The axial displacement can be corrected by mapping some characteristic points that are present along the core. For this experiment a maximum axial displacement of $(0.1 \mathrm{~mm})$ was estimated between confining pressures of 100 to 2500 psig. 

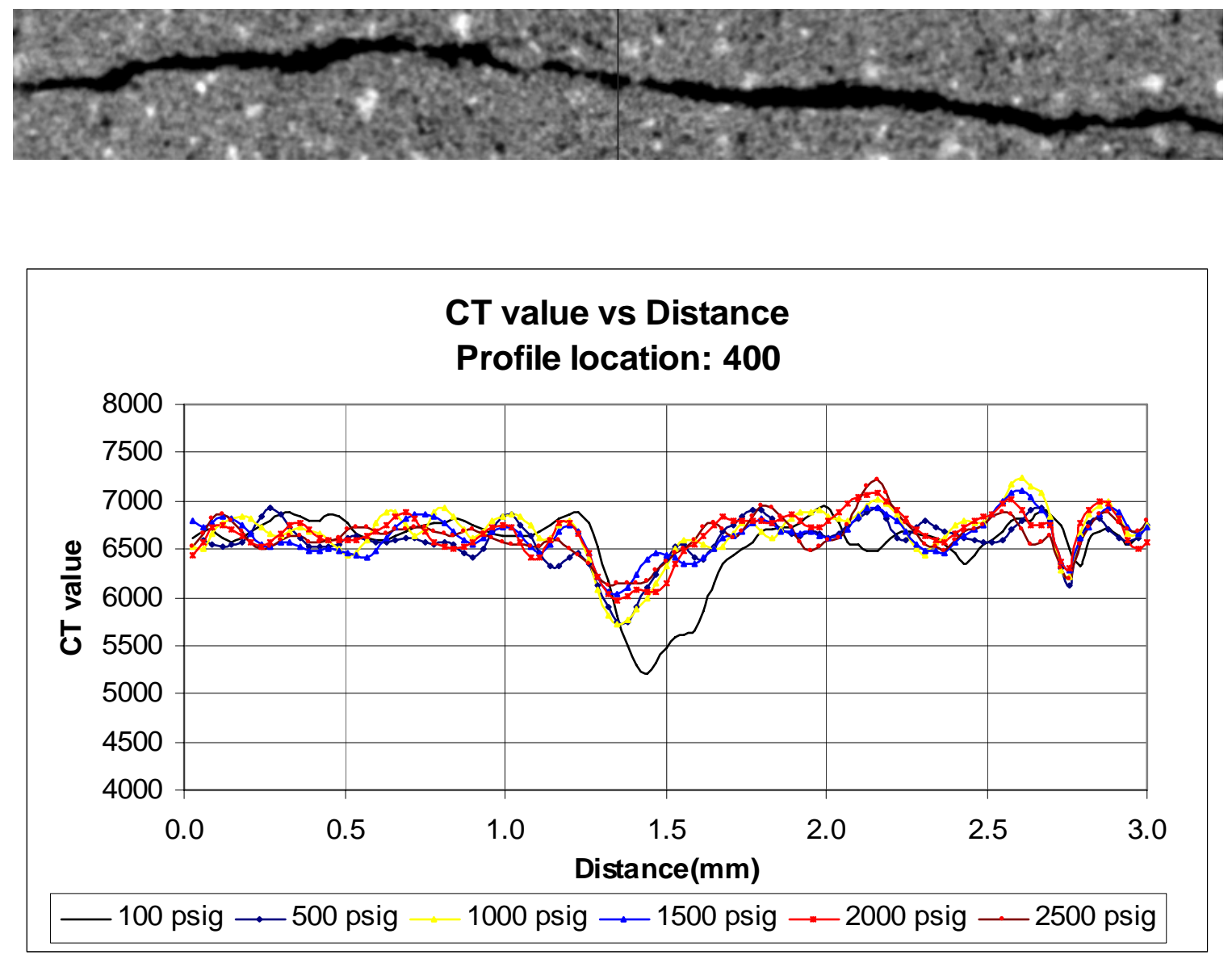

Figure 64: Aperture profiles as a function of confining pressure at the same location in the sample.

Figure 65 shows the fracture aperture (dry scans) in a two dimensional $(24,000 \times 3,000$ microns) plane for different confining pressure from 100 to $2500 \mathrm{psig}$. This sequence of images corresponds to slice number 101 at 3636 microns from the top of the core. The white squares highlight areas where new asperities formed in response to an increase in radial stress. 

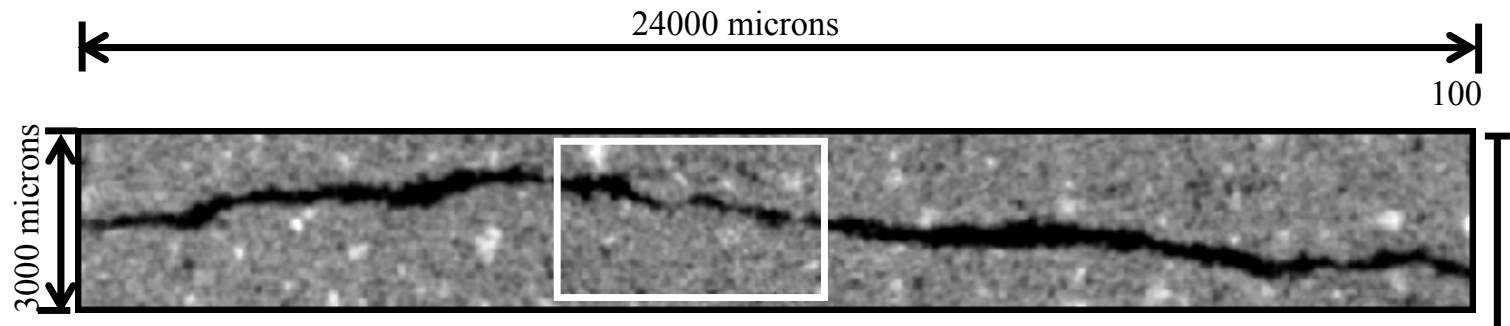

\section{T}

500

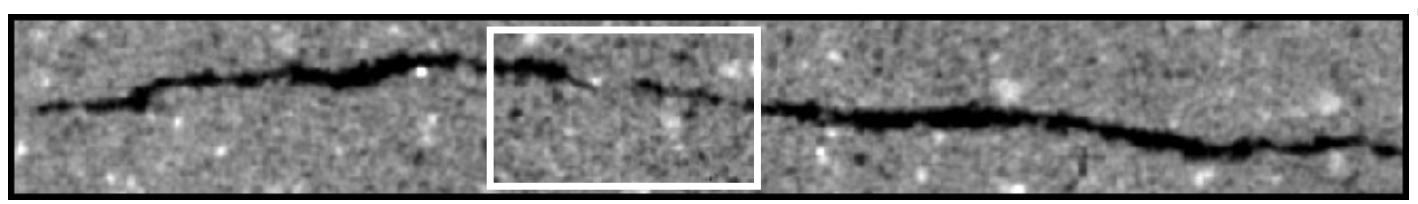

1000

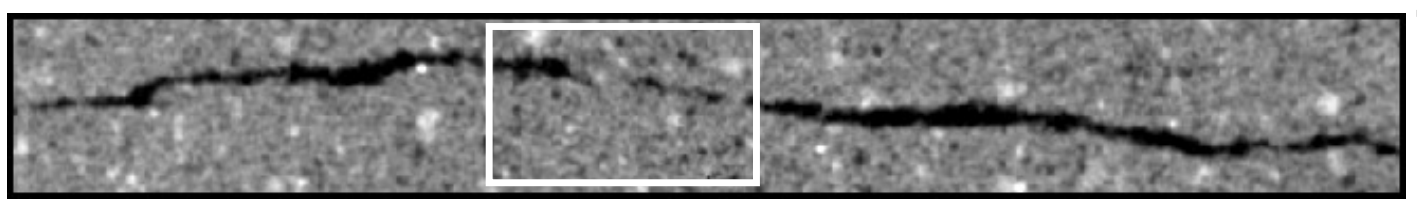

1500

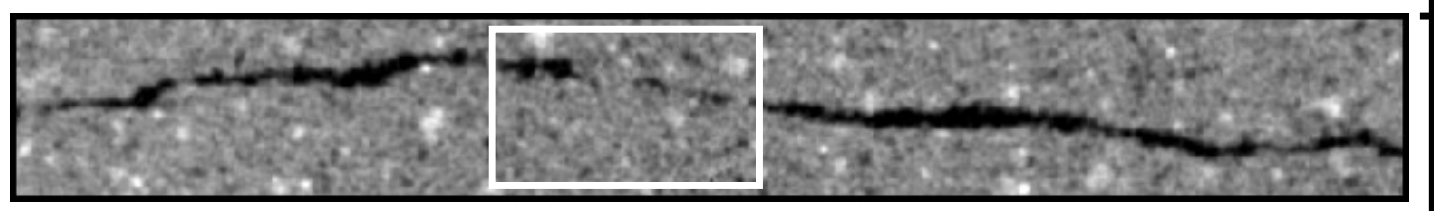

2000

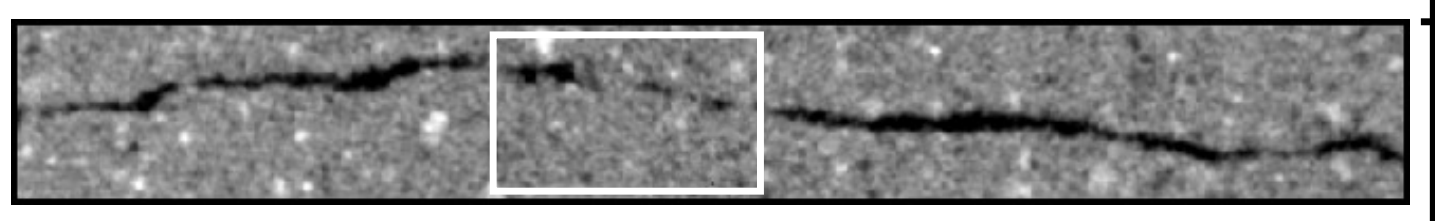

2500

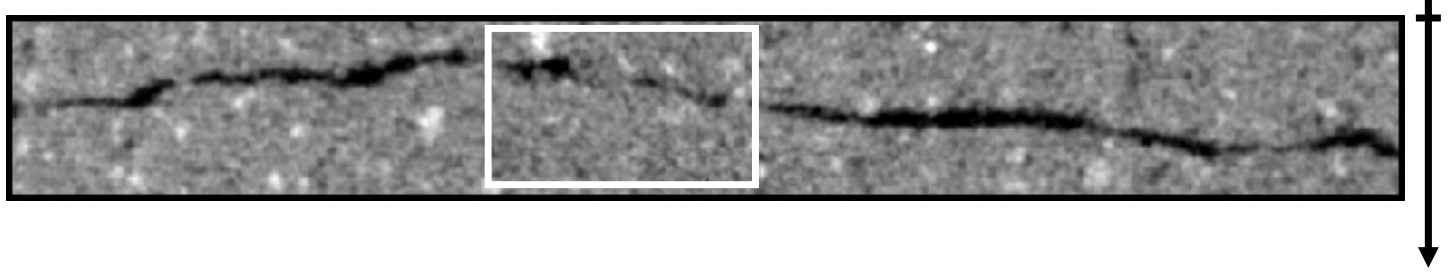

Figure 65: Aperture fracture map for 100, 500100015002000 and 2500

Psig psig confining pressure. 
Figures 66a and 66c show two different CT profiles for two different locations $(4,200$ and 19,800 microns). The initial condition (100 psig), and the final condition (2,500 psig) represent the maximum change in confining pressure. The fracture aperture exchange is represented by a drastic reduction in the CT values. The red circles in Figure 66b and 66c highlight the closure of the fracture (local asperities) in the axial direction. Figure 66b also shows how the flow channels are reduced as a consequence of the stress applied to the core.

The presence of new asperities (contact points) creates pipe-flow channels (Figure 66b). These channels appeared in the two dimensional plane (x-y) and need to be mapped in the axial direction in order to verify and quantify their influence on the fluid flow. 


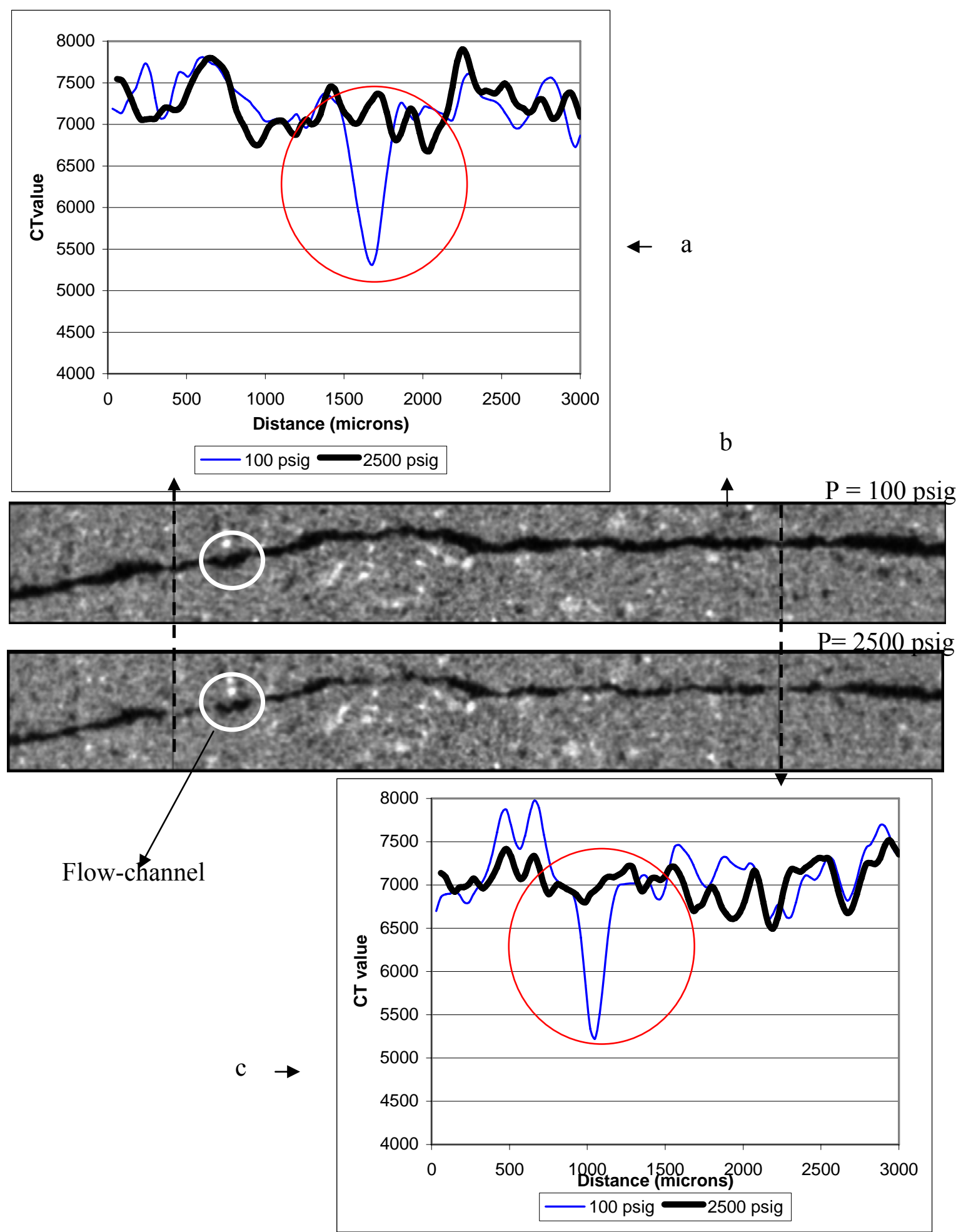

Figure 66: a: CT profile at $\mathrm{x}=140 \mathrm{~b}$ : images for 100 and 2500 psig confining pressure $\mathrm{c}$ : $\mathrm{CT}$ profile at $\mathrm{x}=660$. 
Figures 67a and Figure 67d show CT profiles for Figure 67b, demonstrating the creation of an asperity by the increase in the confining pressure. The CT profiles show an increment in the CT value from 5000 to 6500 (matrix value). The increase in CT value represents a decrease in fracture conductivity. Figure 67c shows a zoom from a secondary fracture at 100 and 2500 psig. The increase in stress closes the fracture and reduces its permeability. This aperture reduction is shown in CT profiles for two of the confining pressure stages, 500 and 2500 psig.

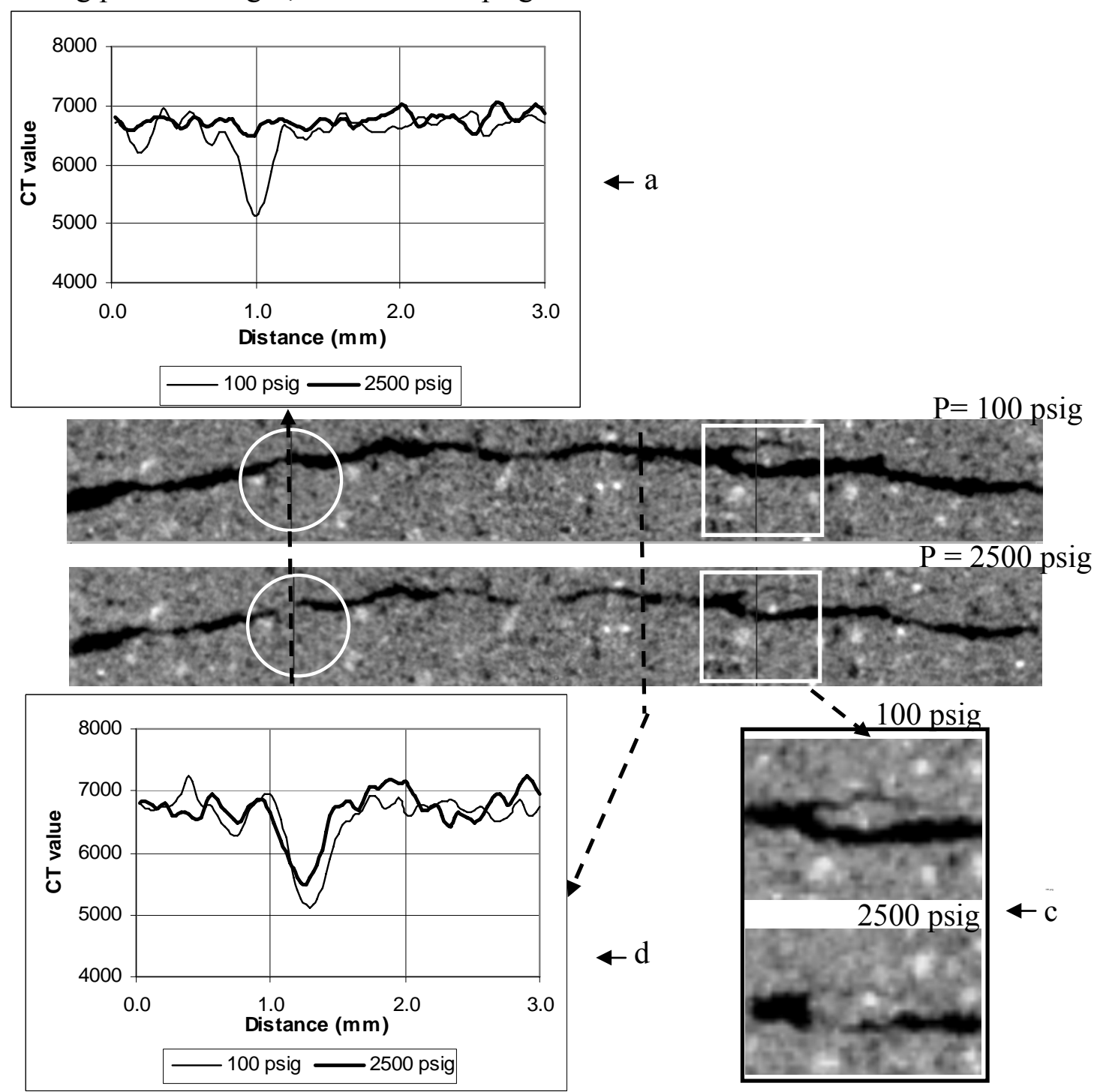

Figure 67: CT profiles at different locations (100 psig and 2500 psig confining pressure). 
After the original CT image volume scans were reduced to boxes $(800 \times 100 \times 410$ or $850 \times 120 \times 2204)$ that contain the fracture, the fracture was characterized by mapping the void space in the sample. Fracture aperture maps and fracture volumes were computed. This process was applied to different confining pressures. Figure 68 shows two threedimensional renditions of the fracture (in red) surrounded by the matrix. Multiple bedding planes can also be observed.
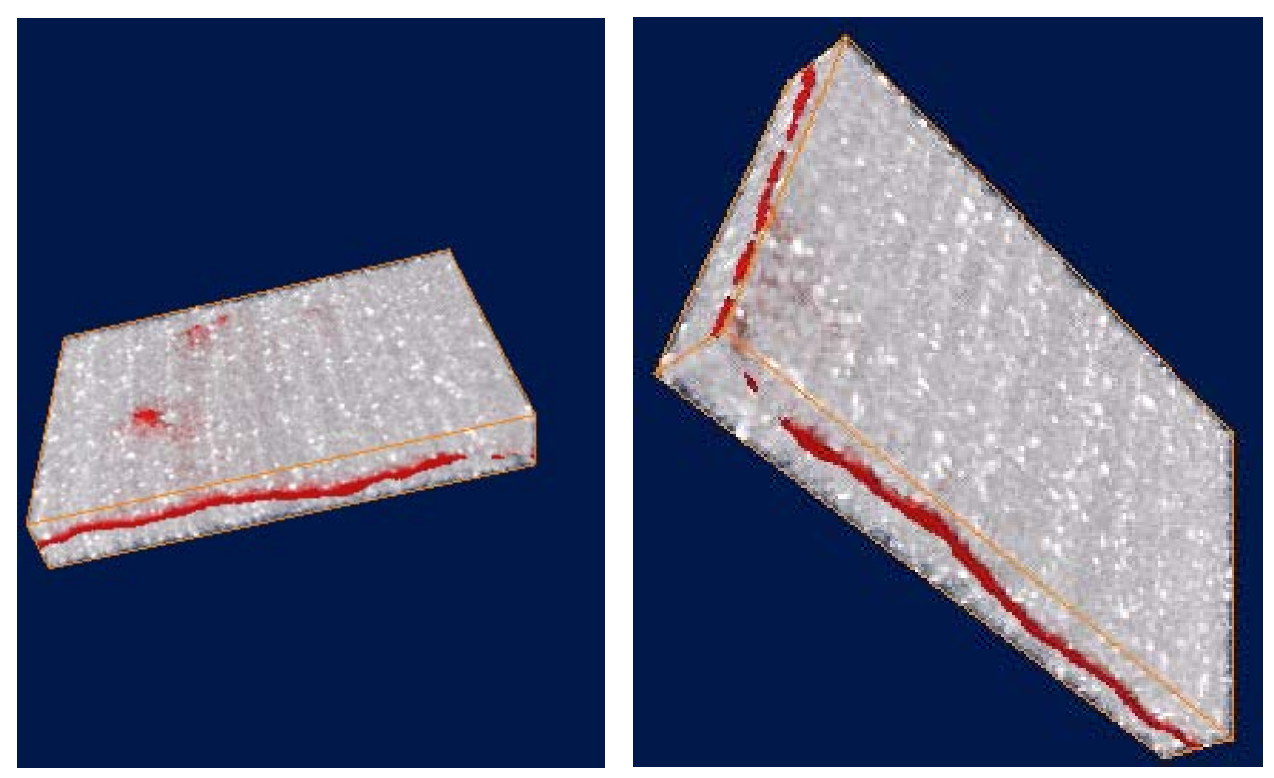

Figure 68: Fracture aperture surrounded by matrix.

Figure 69 presents an iso-surface of the fracture at 100 psig confining pressure (left) and at 2500 psig (right). The increased black regions in Figure 69 (right) remarks the increment in the area of asperities due to the increase in confining pressure. In order to differentiate the fracture from the matrix a threshold must be applied. 
The threshold value was selected carefully by visual examination of the data. The dark color in Figure 69 represents the asperities. These asperities are fracture contact points and are responsible for the reduction of the conductivity of the fracture.
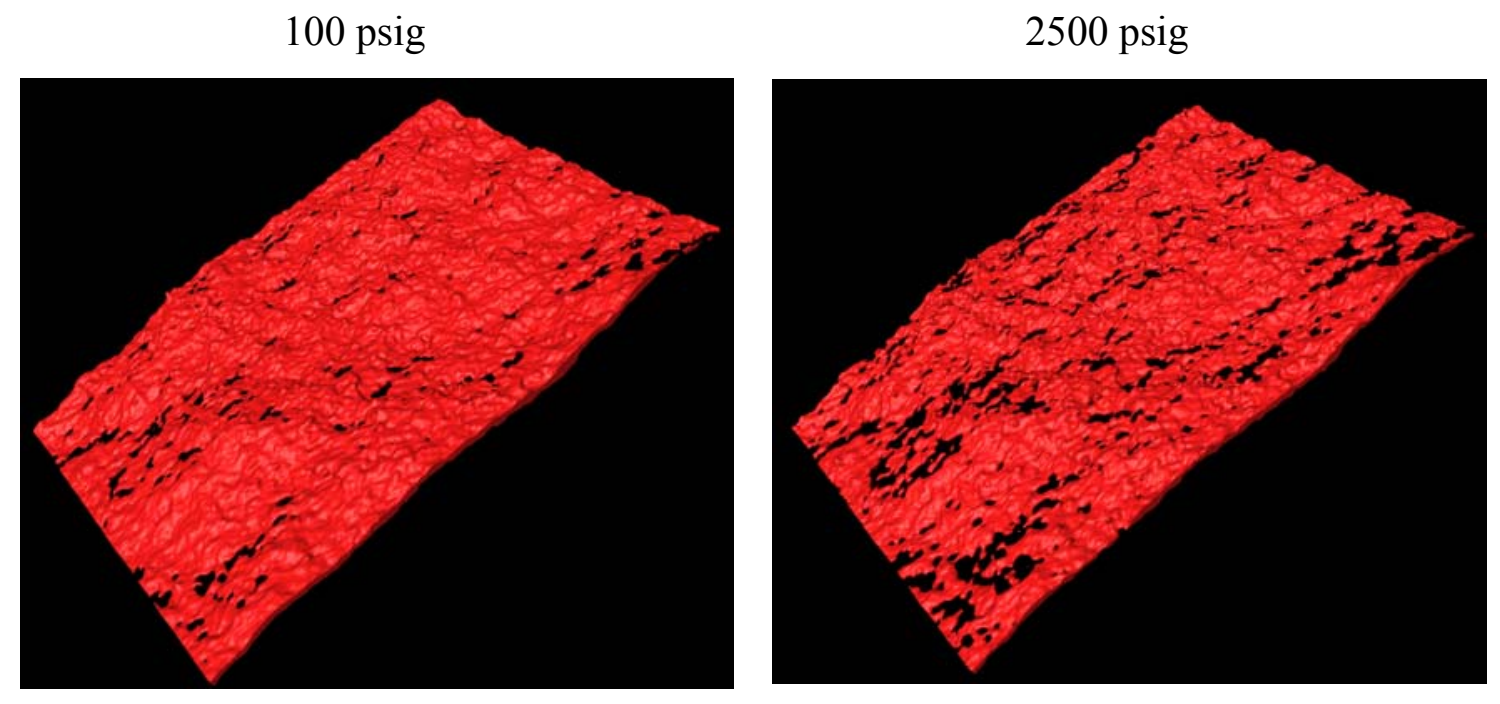

Figure 69: Three-dimensional images of the fracture under 100 and 2500 psig confining pressures.

Figure 70 shows a three-dimensional rendition of the fracture volume for the 100 psig (left) and 2500 psig (right). A coronal slice $(Y=50)$ shows the undulation of the fracture aperture along the core, emphasizing of the non uniform propagation (wave) of the fracture during the fracturing process. Figure $\mathbf{7 0}$ also highlights an increment in area of the walls of the fractures after increasing the radial stress. 

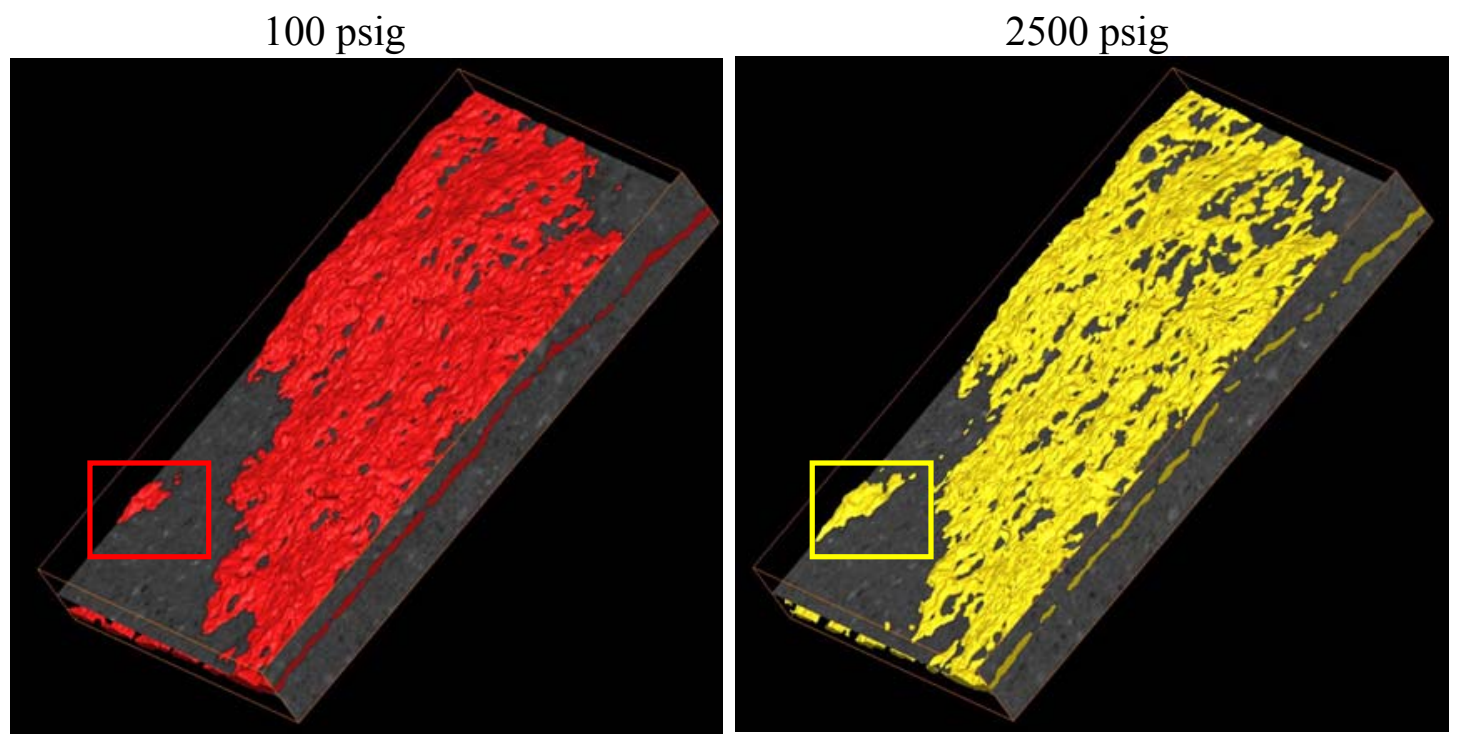

Figure 70: Fracture aperture volume with coronal slice planes.

Once the fracture aperture was partitioned (binary) only fracture and solid phases are left in the system. Figure 71 shows a schematic representation of the fracture, which is the base for the computation of fracture aperture. The zeros represent the matrix and the ones the fracture aperture.

\begin{tabular}{rllllllll|}
\hline \\
\hline
\end{tabular}

Figure 71: Cartoon of the three-dimensional (binary) fracture aperture array. 
The $\mathrm{Y}$-direction dimension was 30 microns and is taken to be orthogonal to the fracture and is used in calculating fracture aperture. Figure 72 presents 2D fracture aperture maps at 100 psig and 2500 psig.

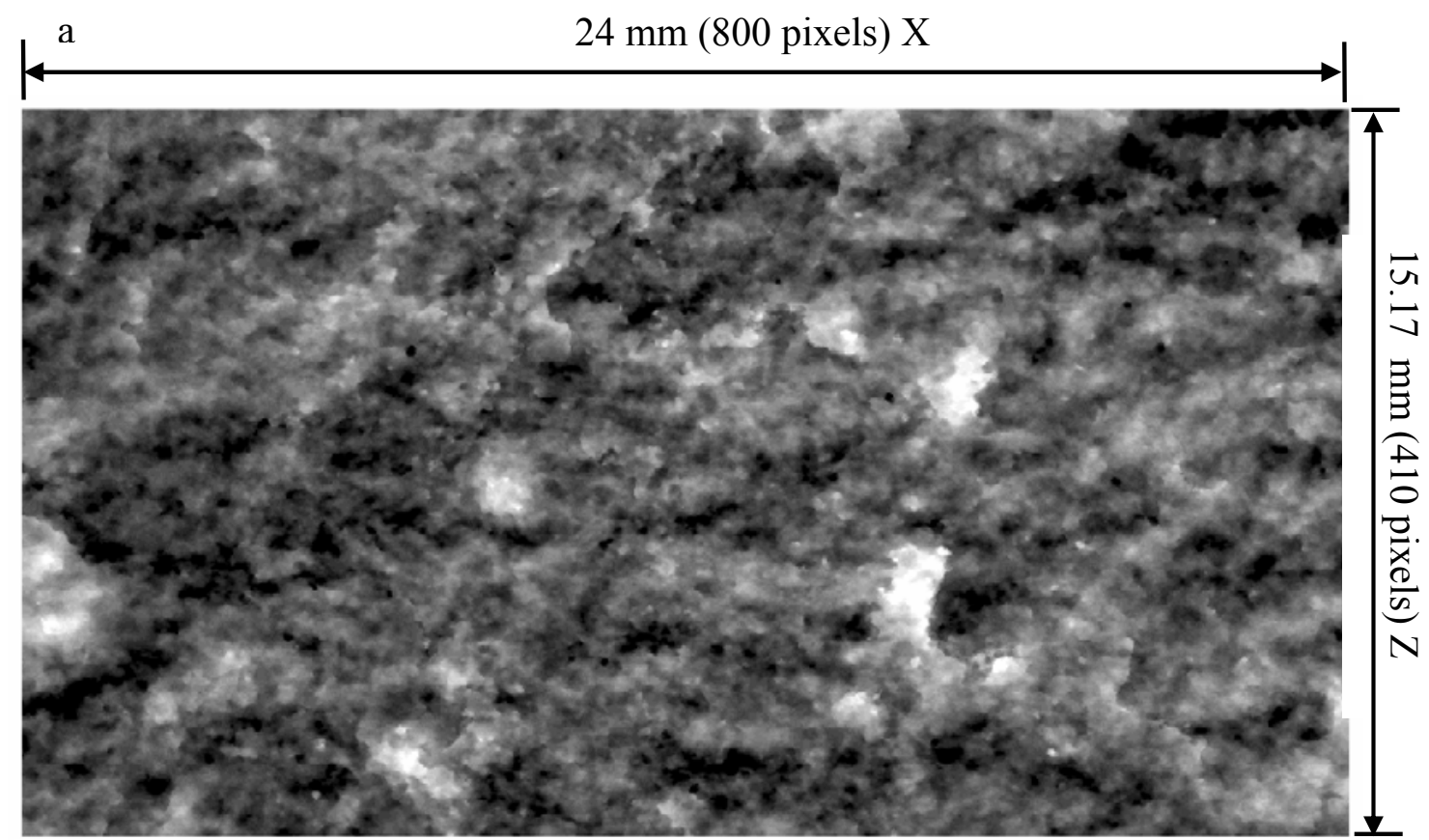

$\mathrm{b}$

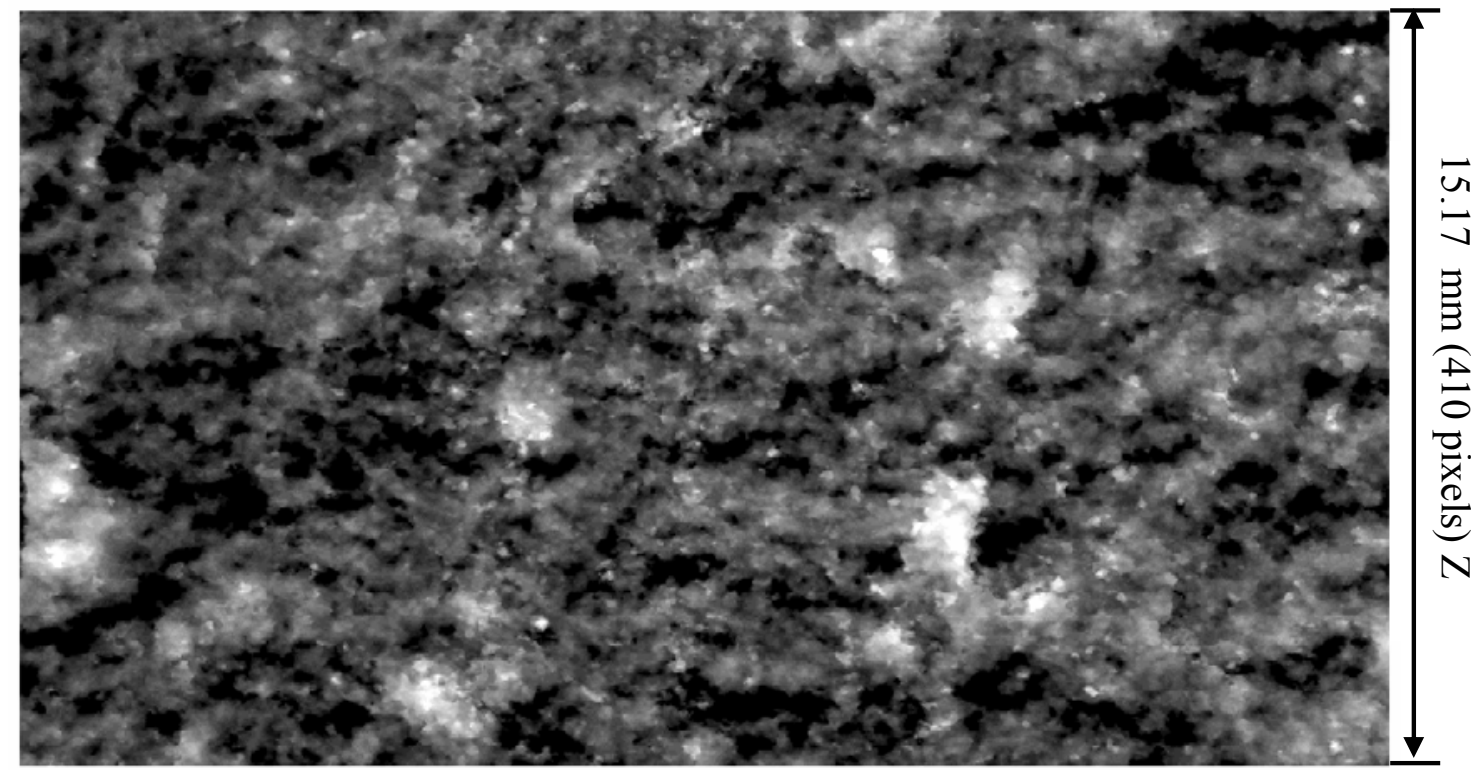

Figure 72: Two dimensional fracture aperture maps. a: 100 psig. b: 2500 psig. 
The light color highlights high porosity areas (high fracture aperture). The top image corresponds to 100 psig while bottom to 2500 psig. Figure 73 highlights the increment in the number of asperities, where red color corresponds to places with zero fracture aperture (less than 30 microns or 1 voxel).

The computed fracture aperture volume was 99.4 cubic millimeters with $1.58 \%$ of the area occupied by asperities at 100 psig, and 83.2 cubic millimeters with $5.69 \%$ asperities for the case of 2500 psig. Figure 74 shows the fracture aperture probability distribution (2500 psig) with a maximum probability value of $11.23 \%$ for fracture aperture in the range of 210 to 240 microns. The average fracture aperture was 273 microns for the 100 psig confining pressure and 229 microns for the 2500 psig confining pressure, an over reduction of $15 \%$. 


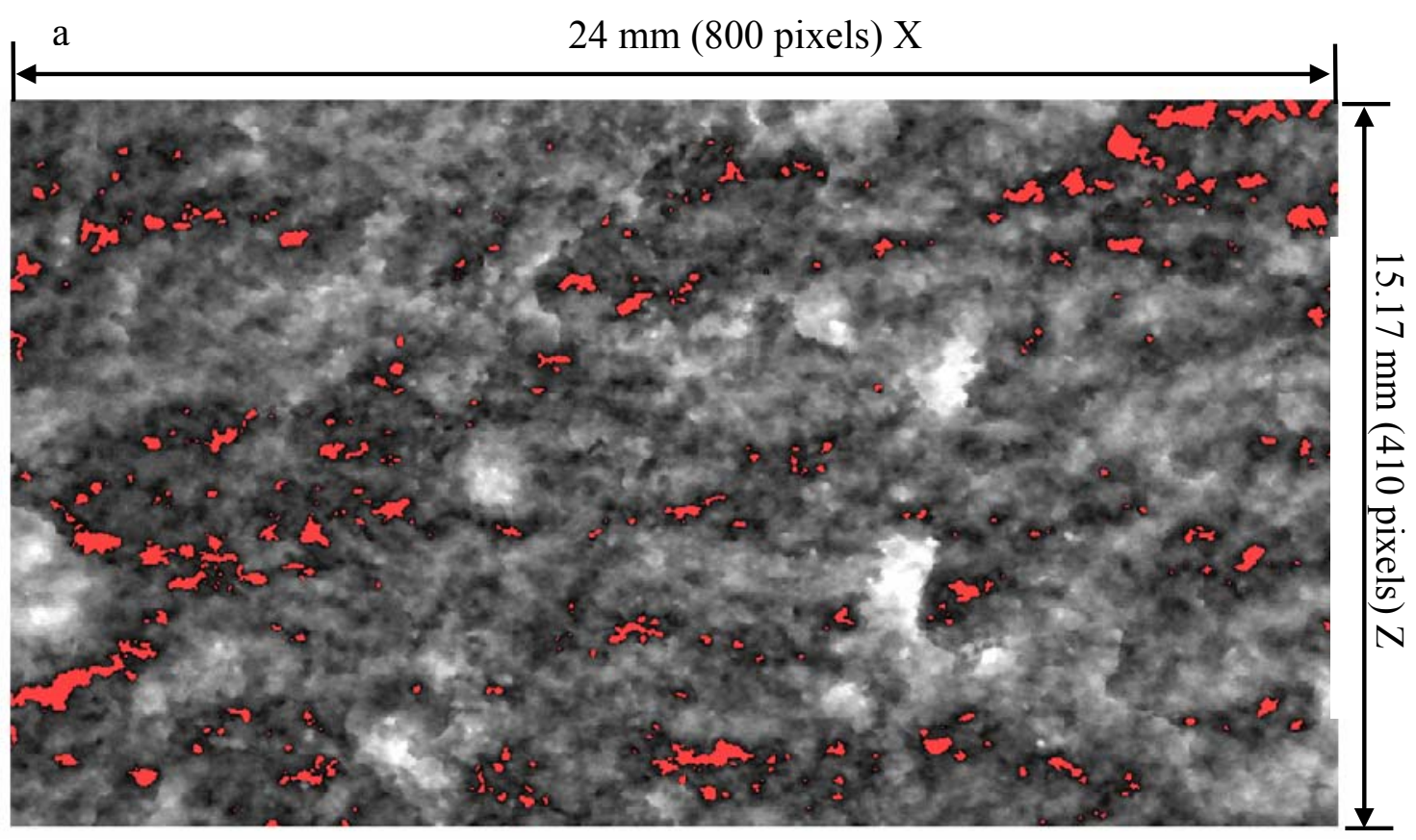

b

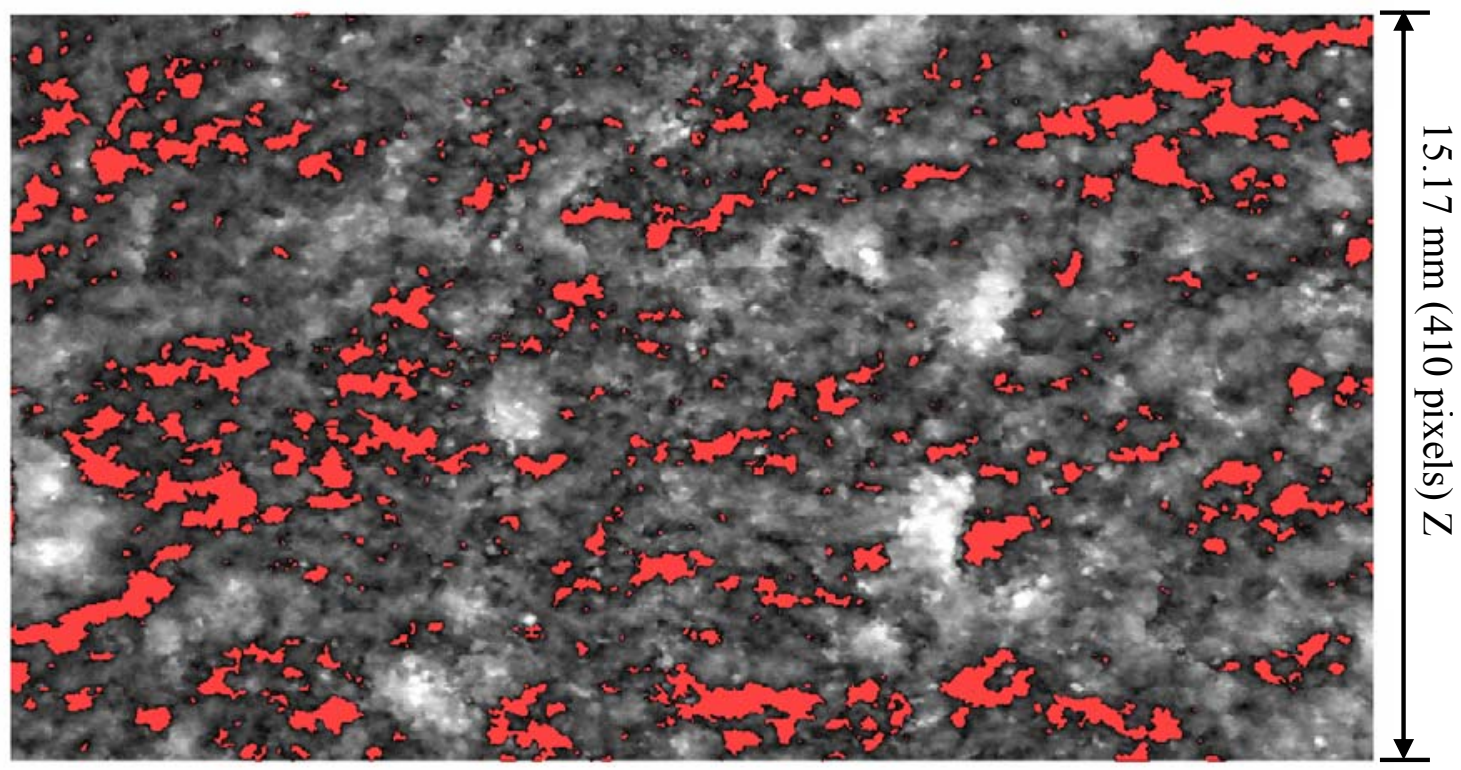

Figure 73: Two dimensional asperities maps. 


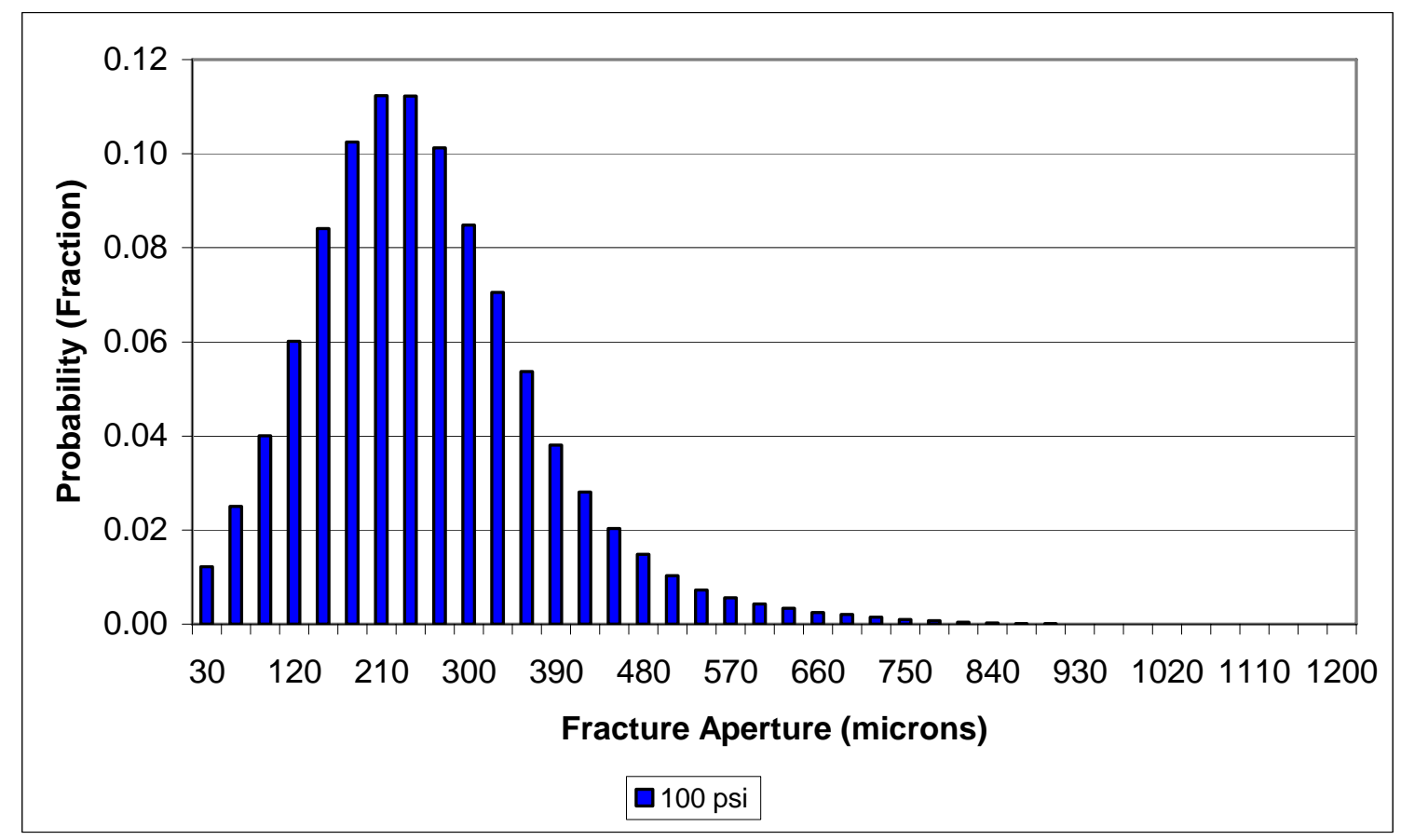

Figure 74: Probability plot from the fracture aperture.

Figure 75 shows the probability density function against the fracture aperture $(2500$ psig). Earlier studies, [Gale (1987), Pyrak-Nolte et al. (1987)] have suggested lognormal or gamma functions for fracture aperture distributions, highlighting the importance of the tail of such distributions that represents channel flow. Therefore, it is extremely important to characterize the fracture aperture not only based on the fracture porosity but also based on the interconnectivity of the fracture and the possibility of having flow channels. 


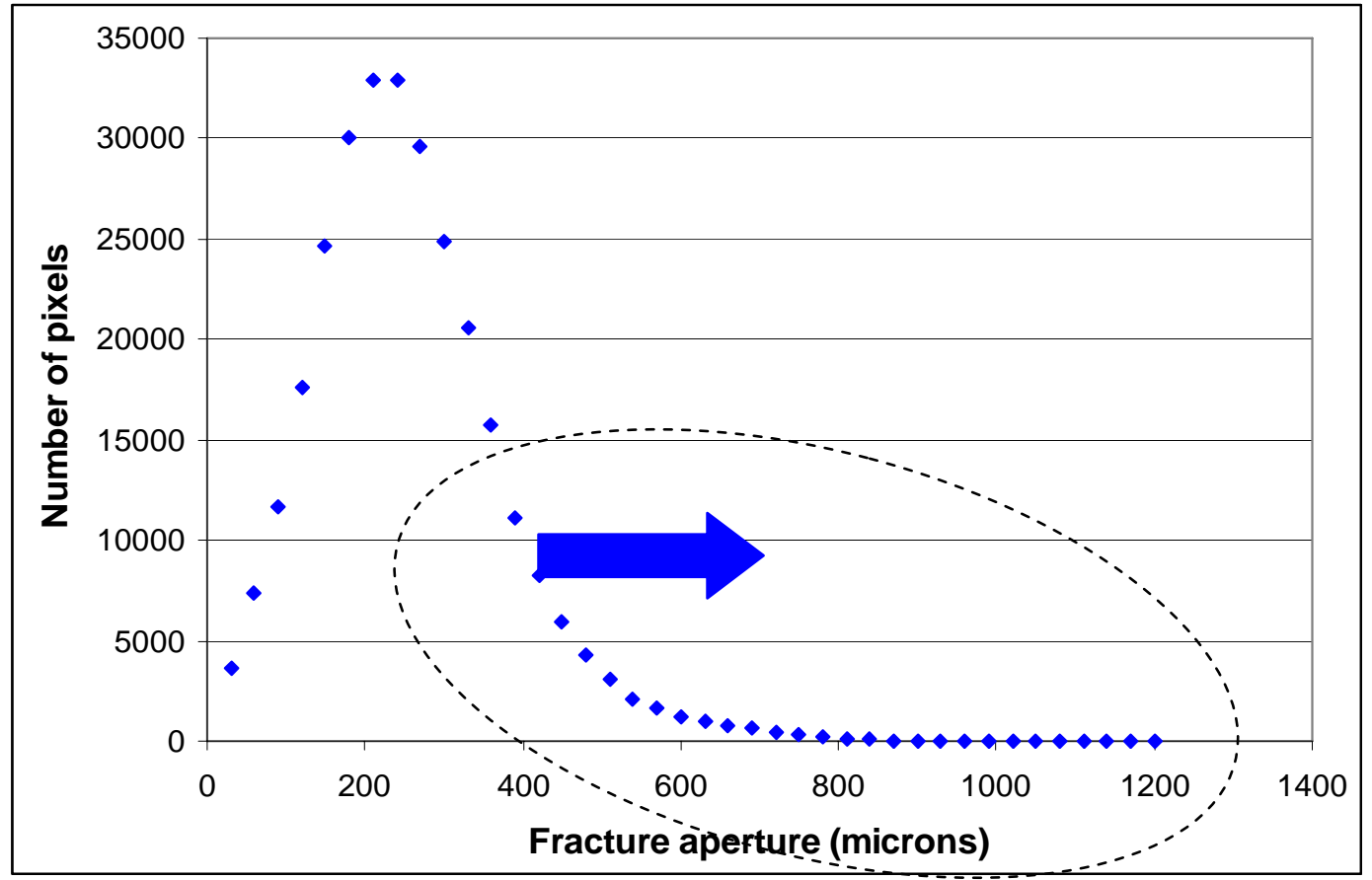

Figure 75: Fracture aperture distribution (2500 psig).

Figure 76 presents the fracture aperture distribution for the 100 and 2500 psig. The 2500 psig curve is axially displaced (down) and shifted to the left indicating the reduction of fracture aperture.

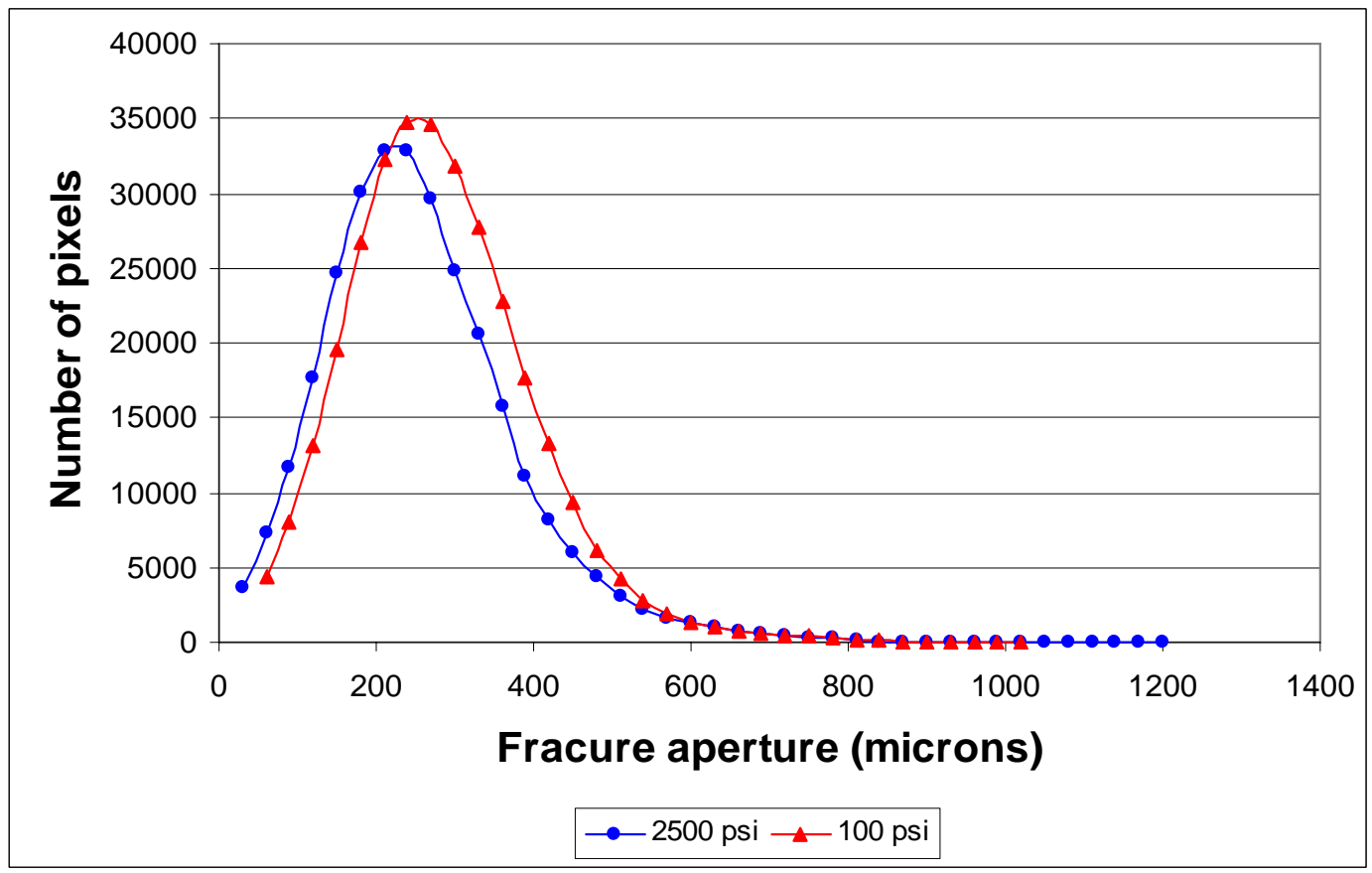

Figure 76: Fracture aperture distributions for 100 and 2500 psig. 
Using the fracture aperture distribution Zimmerman et al. (1991) suggested that the roughness of the fracture can be described or represented by the ratio of the standard deviation and the mechanical aperture of the fracture aperture distribution:

Roughness Factor $=\frac{\sigma_{m}}{a_{m}}$

Where $\sigma_{m}$ represents the standard deviation and $a_{m}$ the mechanical aperture.

A perfect parallel model has uniform fracture aperture and therefore a zero standard deviation, leading to a zero roughness factor. For the 100 psig and 2500 psig confining pressure the roughness factors were calculated. Table 8 shows the computed values, where the 2500 psig shows a higher deviation from the zero indicating an increase in roughness in the fracture as a consequence of the increase in the area of the asperities and the reduction in the average fracture aperture. Figure 77 presents a volume fracture aperture map. The black color represents no flow (asperities) and the red color represents open fracture (potential flow paths

Table 8: Roughness factor values

\begin{tabular}{|c|c|c|c|}
\hline $\begin{array}{c}\text { Confining } \\
\text { Pressure (psi) }\end{array}$ & $\begin{array}{c}\mathrm{a}_{\mathrm{m}} \\
\text { (microns) }\end{array}$ & $\begin{array}{c}\sigma_{\mathrm{m}} \\
\text { (microns) }\end{array}$ & Roughness Factor \\
\hline 100 & 281 & 281 & 1.06 \\
\hline 2500 & 351 & 256 & 1.32 \\
\hline
\end{tabular}




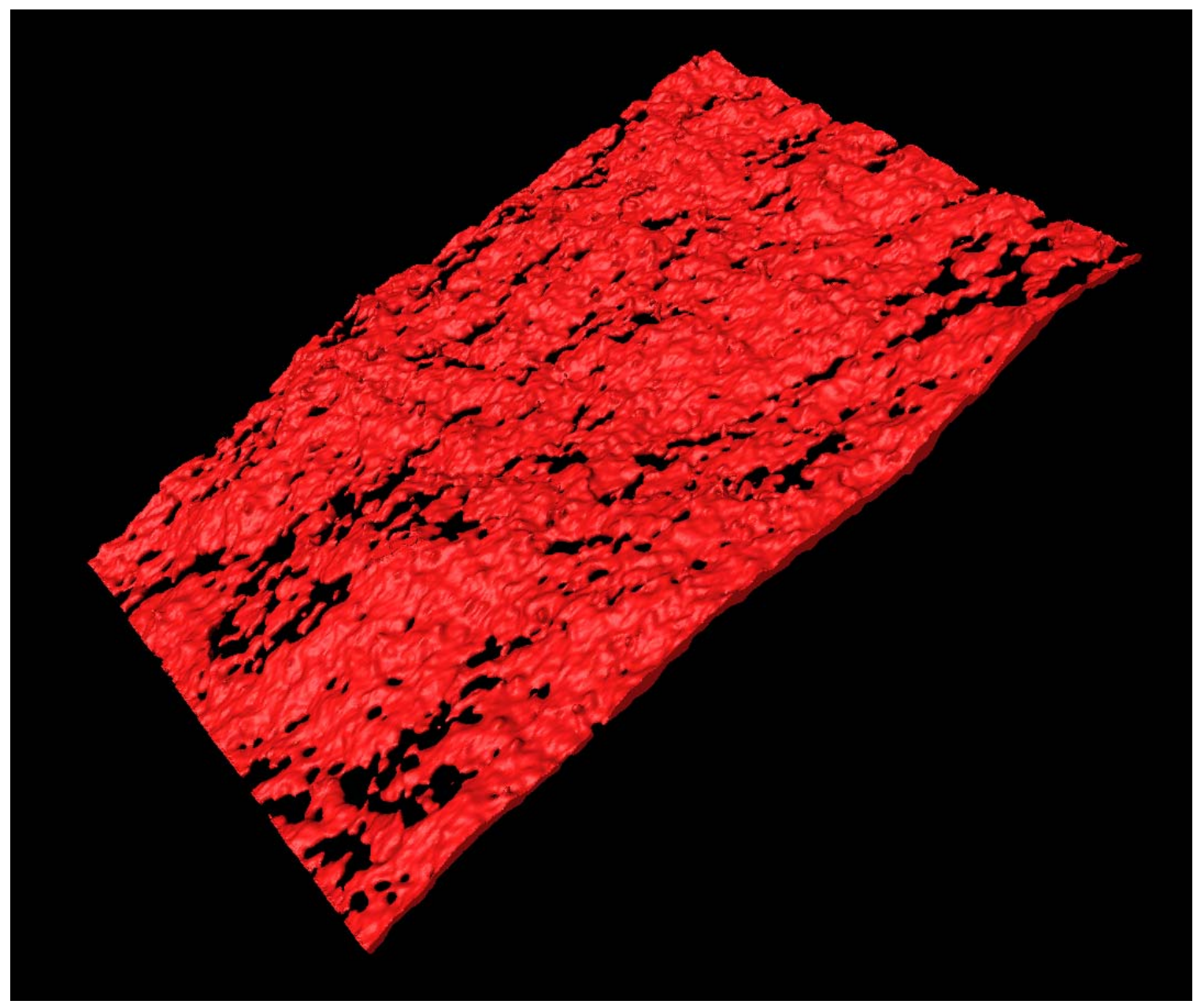

Figure 77: Three dimensional representation of fracture aperture at $2500 \mathrm{psig}$.

In order to analyze the conductivity of the fracture the tail of the fracture was extracted from the fracture aperture data. Figure 78 shows potential flow paths in the fracture, where the yellow regions highlight regions where the fracture aperture is grater than 360 (12 pixels) microns. These regions correspond to the tail of the fracture distribution shown in Figure 75. 


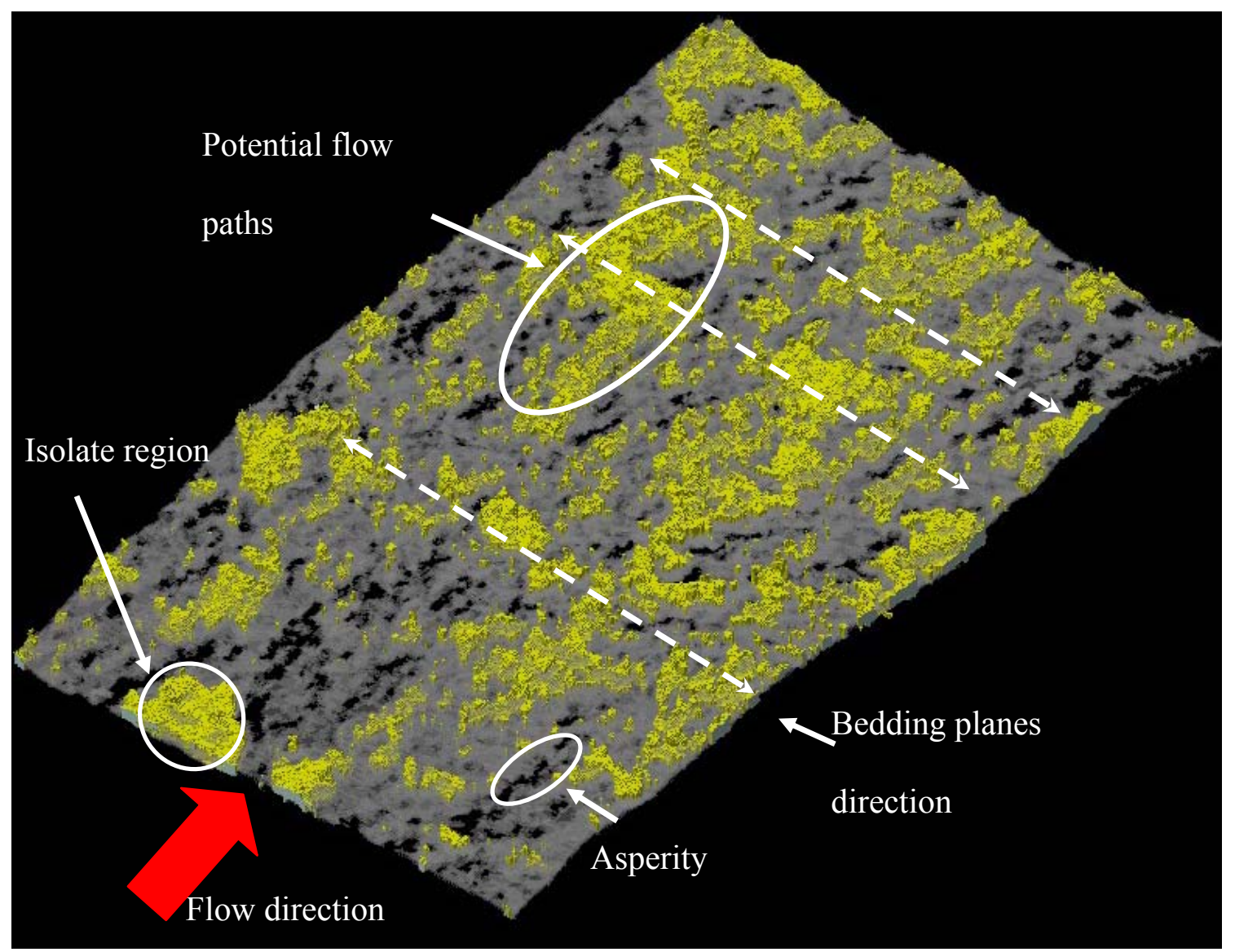

Figure 78: Fracture aperture distribution 360 microns thickness or more.

From Figure $\mathbf{7 8}$ it is also possible to identify isolated regions (white circle on Figure 78) that do not contribute to the fluid flow. Few flow channels in the bedding plane direction (perpendicular to fracture plane) can be observed. 
Structure Model Index (SMI): A Structure Model Index has been used to characterize the fracture aperture. Hildebrand et al. (1997) defined a morphometric parameter called Structure Model Index (SMI). The SMI parameter is used to quantify the characteristics form of a three-dimensional structure in terms of the amount of plates and rod composing the structure. This technique is base on the differential analysis of the triangulate surface and was implemented in bones for finding the deterioration of cancellous bone structure due to diseases. The SMI index parameter varies from 0,3 , and 4 corresponding to objects that have the shapes of plates, rods, and spheres, respectively. The Structure Model Index is given by Equation 5.1:

$$
S M I=6\left[\frac{S^{\prime} V(r)}{S(r)^{2}}\right]
$$

Where $S^{\prime}=\frac{\partial S}{\partial r},(\mathrm{r})$ represents the normal direction to the surface of the fracture and $S^{\prime}$ is the derivative of the surface area with respect to the orthogonal direction (r). V and $\mathrm{S}$ represent the fracture volume and surface area respectively. $S$ ' is computed by creating a displacement of the original surface incrementally by a factor $\Delta \mathrm{r}$ and evaluating the new surface are at the $\mathrm{r}+\Delta \mathrm{r}$ position. Equation 5.2 shows the numerical computation of $\mathrm{S}$ ':

$S^{\prime}=\frac{\partial S}{\partial r}=\frac{S(r+\Delta r)-S(r)}{\Delta r}$ 
Hildebrabd and Ruegsegger suggested a $\Delta \mathrm{r}$ value between 0.001 and 0.1 of the element edge voxel. The displacement used in this work corresponds to a ratio of 0.01 times the $\operatorname{volxel}$ size $(\Delta x=\Delta y)$ for a $\Delta \mathrm{r}$ equal to $0.00026 \mathrm{~mm}$.

Table 9 highlights the numerical computation of the Structure Model Index for Core BS2 at two different confining pressures, 500 and 2500 psig. The increase of the SMI highlights the closure and deformation of the fracture, where new non connected channels have been created.

Table 9: SMI parameters for 500 and 2500 psig.

\begin{tabular}{|c|c|c|c|c|c|c|c|}
\hline & \multicolumn{6}{|c|}{ Coefficients } & \\
\hline $\begin{array}{c}\text { Pressure } \\
\text { (psig) }\end{array}$ & $\begin{array}{c}\text { So }(\mathrm{r}) \\
\left(\mathrm{mm}^{2}\right)\end{array}$ & $\begin{array}{c}\mathrm{S} 1(\mathrm{r}+\Delta \mathrm{r}) \\
\left(\mathrm{mm}^{2}\right)\end{array}$ & $\begin{array}{c}\text { Vo } \\
\left(\mathrm{mm}^{3}\right)\end{array}$ & $\begin{array}{c}\mathrm{V} 1 \\
\left(\mathrm{~mm}^{3}\right)\end{array}$ & $\Delta \mathrm{r}(\mathrm{mm})$ & S $^{`}$ & SMI \\
\hline Fracture 500 & 4216.78 & 4219.03 & 373 & 374 & 0.00026 & 8654.6 & 1.090567 \\
\hline Fracture 2500 & 3777.83 & 3780.92 & 278 & 279 & 0.00026 & 11884.6 & 1.388956 \\
\hline
\end{tabular}

Figure 79 shows the SMI coefficient for a series of changes in confining pressure from 100 to 2500 psig (core BS1). At low pressure a significant SMI increment is observed $(\sim 0.37)$. However, when the confining pressure goes up the SMI is asymptotic to a value of 2.5. This behavior is consequence of the small reduction of the volume fracture related to the changes in surface area, consequence of the induced asperities in the system. 


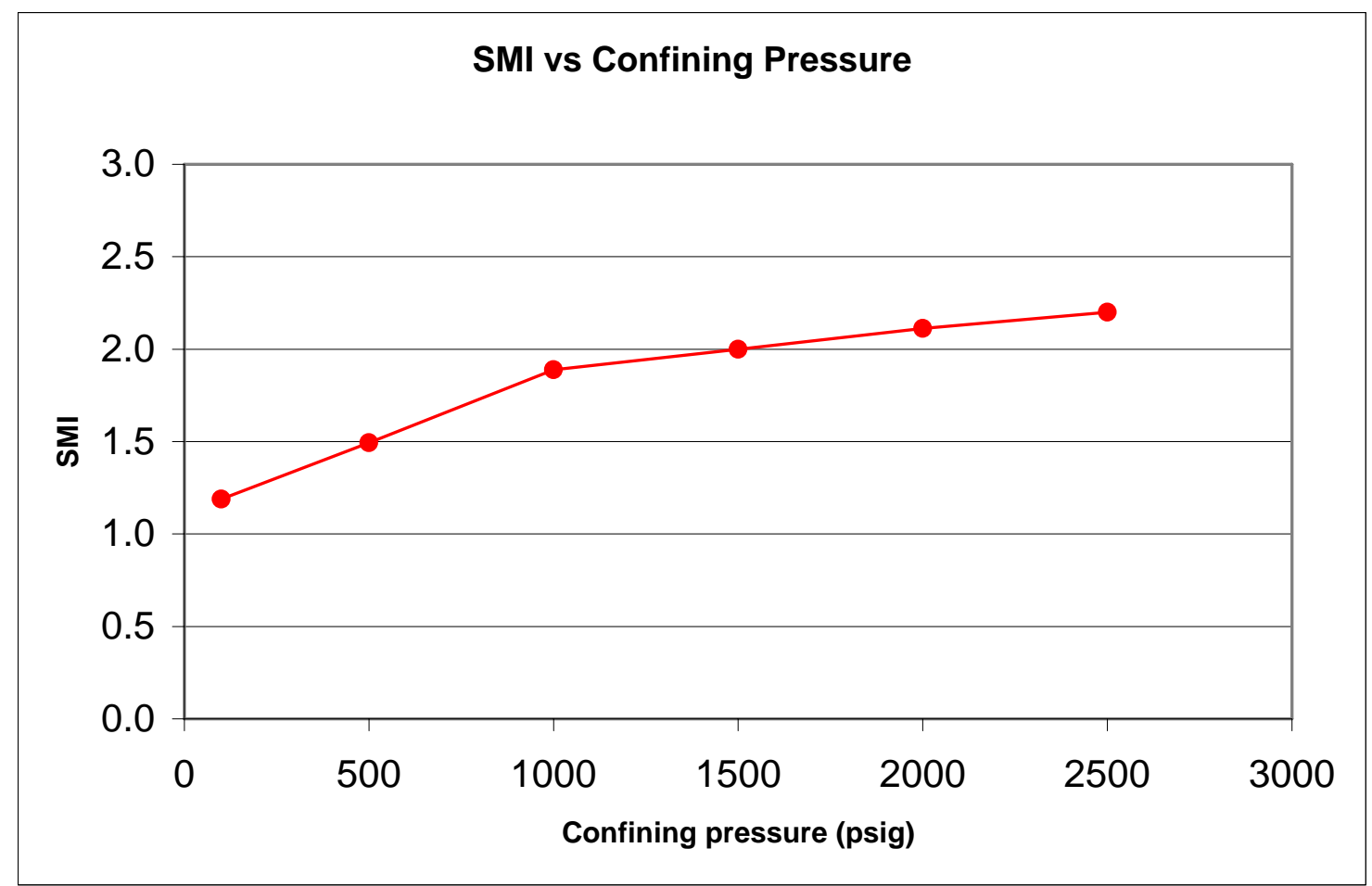

Figure 79: Structure model index for different confining pressure.

Connectivity density coefficient (EPC): The Euler-Poincaré coefficient (EPC) was computed for the fracture structure. This coefficient is a measure of the connectivity density (connections per unit volume). The Euler-Poincaré characteristic of a threedimensional structure is a topological invariant, which reports the number of particles of a structure plus the number of enclosed cavities minus the connectivity. This technique has been used for the quantification of connectivity in bones and is summarized by Odgaard et al. (1993) This method uses the number of voxels faces, edges, and corners associated with a three dimensional object that is composed of voxels. 
Prior to computing the EPC, the fracture was segmented by applying a threshold (2400). A binary system was created, where the number one was assigned to the fracture pore space and the number zero to the matrix volume. The dimensions (X, Y and, Z) of the entire sample containing the fracture were $20.8 \times 3.12 \times 63.49 \mathrm{~mm}$ or $800 \times 120 \times 2048$ (voxel size), where the volex sizes were $\mathrm{dx}=\mathrm{dy}=0.026 \mathrm{~mm}$ and $\mathrm{dz}=0.031 \mathrm{~mm}$. The three dimensional fracture volume sub-divided (gridding) in the $\mathrm{x}$ and $\mathrm{z}$ direction, keeping the aperture direction of the fracture (y) constant and equal to $3.12 \mathrm{~mm}(120$ voxels). Once the fracture was sub-divided into different blocks an EPC was computed for each block. Figure 80 shows the dry fracture at 2500 psig (left) and the subdivision where the fracture sample was divides into four equal sub-blocks ( $2 \times 2$ case). Other subdivisions included were 16 blocks (4x4), 64 blocks (8x8), and 256 blocks (16x16).

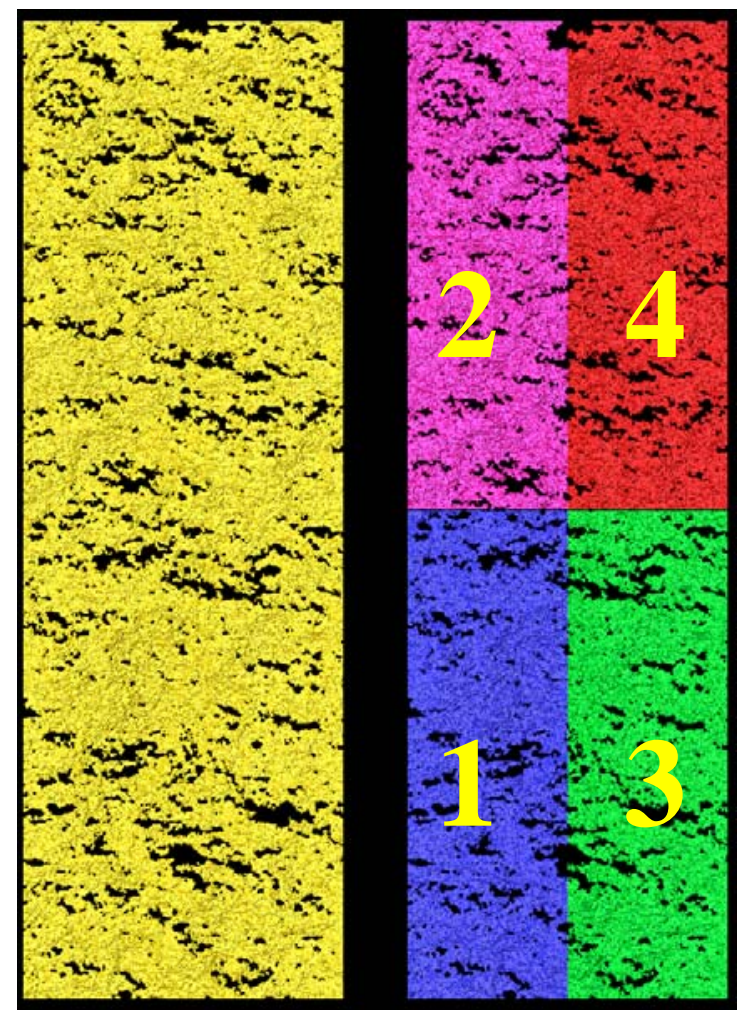

Figure 80: Fracture sub-division scheme into 4 blocks (2x2). 
Table 10 highlights the dimension of each block during the fracture refining process. The EPC and SMI results are summarized in Table 11.

Table 10: Summary of the segmentation process and block dimensions

\begin{tabular}{|l|c|c|c|c|c|}
\hline Grid & Total & $\mathbf{d x}(\mathbf{m m})$ & $\mathbf{d y}(\mathbf{m m})$ & $\mathbf{d z}(\mathbf{m m})$ & Voxels \\
\hline $\mathbf{1 x 1}$ & 1 & 20.800 & 3.120 & 63.488 & $800 \times 120 \times 2048$ \\
\hline $\mathbf{2 x 2}$ & 4 & 10.400 & 3.120 & 31.744 & $400 \times 120 \times 1024$ \\
\hline $\mathbf{4 x 4}$ & 16 & 5.200 & 3.120 & 15.872 & $200 \times 120 \times 512$ \\
\hline $\mathbf{8 x 8}$ & 64 & 2.600 & 3.120 & 7.936 & $100 \times 120 \times 256$ \\
\hline $\mathbf{1 6 x 1 6}$ & 256 & 1.300 & 3.120 & 3.968 & $50 \times 120 \times 128$ \\
\hline
\end{tabular}

Table 11: Summary of SMI and Euler-Poincaré values (Averages)

\begin{tabular}{|l|c|c|c|c|}
\hline & \multicolumn{2}{|c|}{500 psig } & \multicolumn{2}{c|}{ 2500 psig } \\
\hline & Euler-Poincaré & SMI & Euler-Poincaré & SMI \\
\hline $\mathbf{1 x 1}$ & 0.325 & 1.963 & 0.432 & 2.275 \\
\hline $\mathbf{2 x 2}$ & 0.323 & 2.423 & 0.431 & 2.482 \\
\hline $\mathbf{4 x 4}$ & 0.323 & 2.334 & 0.430 & 2.371 \\
\hline $\mathbf{8 x 8}$ & 0.322 & 1.554 & 0.429 & 1.769 \\
\hline $\mathbf{1 6 x 1 6}$ & 0.315 & 1.288 & 0.425 & 1.593 \\
\hline
\end{tabular}


An increase in the Euler-Poincaré number for the change in confining pressure was observed. This increase in connection density is a consequence of creating new asperities. Figure 81 shows the distribution of Euler-Poincaré values for the $16 x 16$ case at 500 psig. The computed average value for this case was 0.3157 .

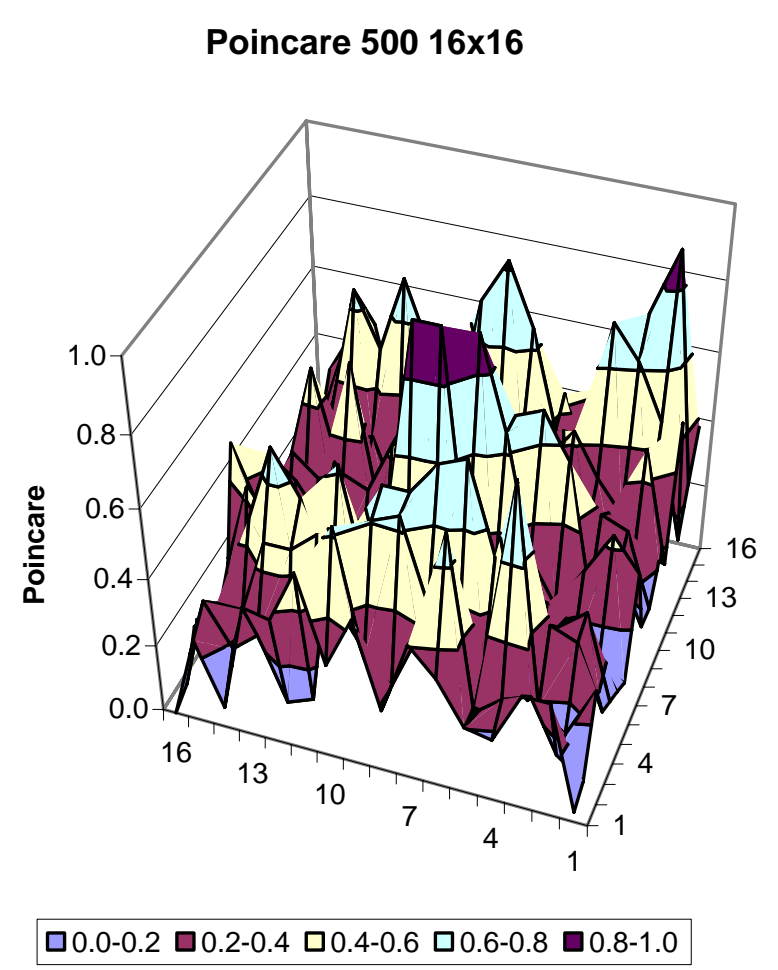

Figure 81: Euler-Poincaré distribution for the 16x16 case at 500 psig.

Figure 82 presents the corresponding Euler-Poincaré graph for the 2,500 psig case (16x16) where the average value was 0.4256 . 


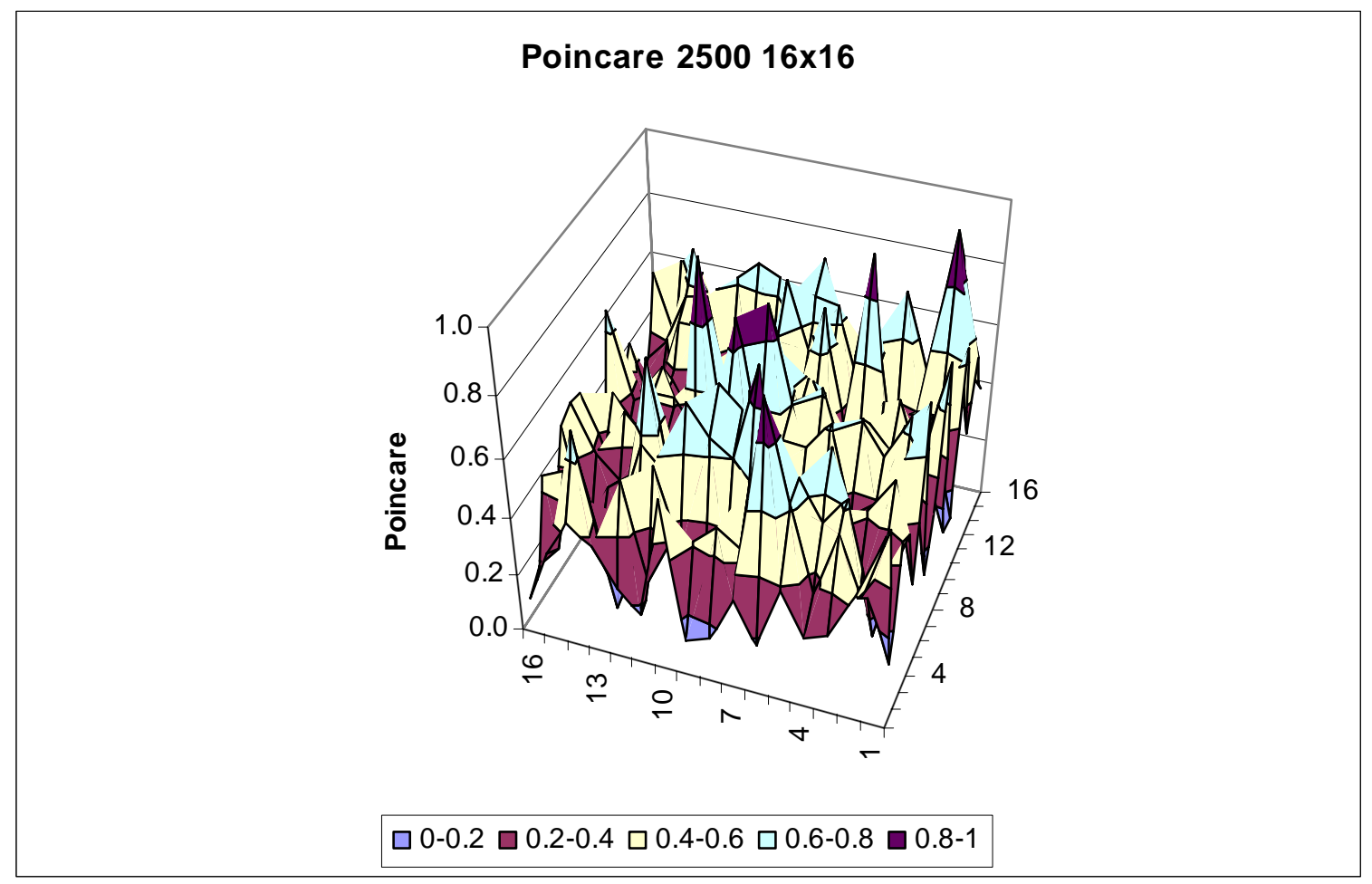

Figure 82: Euler-Poincaré distribution for the $16 \times 16$ case at 2500 psig.

The connectivity changes in the fracture are quantified by variations in connectivity densities (EPC) and for changes in the fracture structure (SMI). Figure 83 shows the SMI changes in the $16 \times 16$ case at 2500 psig, where a SMI average was equal to 1.288 for 500 psig and 1.593 for 2500 psig. Both method, EPC and SMI show agreement from the structural and connectivity point of view, highlighting regions with significant structural changes for both cases, 500 and 2500 psig. 


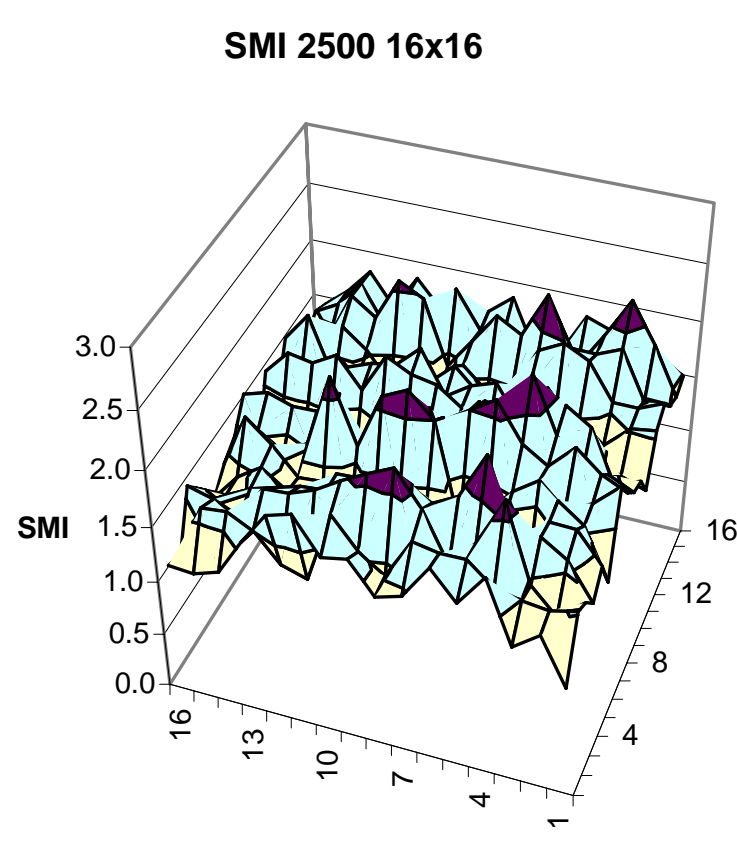

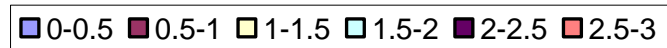

Figure 83: SMI distribution for the $16 \times 16$ case at 2500 psig.

A clear application of the EPC would be to identify fracture characteristic that can be used to simulate flow conditions in micro fractured models. Figure 84 shows blocks 135 and 241 for the $256(16 \times 16)$ case. They were identified as the maximum and minimum Euler-Poincaré number at 500 psig. The block on the Figure 84 (a) has a 0 EPC while the one on the right, Figure 84 (b), has an EPC of 0.947. The block of low EPC value has a smaller volume $\left(1.77 \mathrm{~mm}^{3}\right)$ than the one of high Euler-Poincaré $\left(1.69 \mathrm{~mm}^{3}\right)$. As has been proved previously, an increase in confining pressure creates more asperities that can be correlated with a high EPC (high connectivity density). 


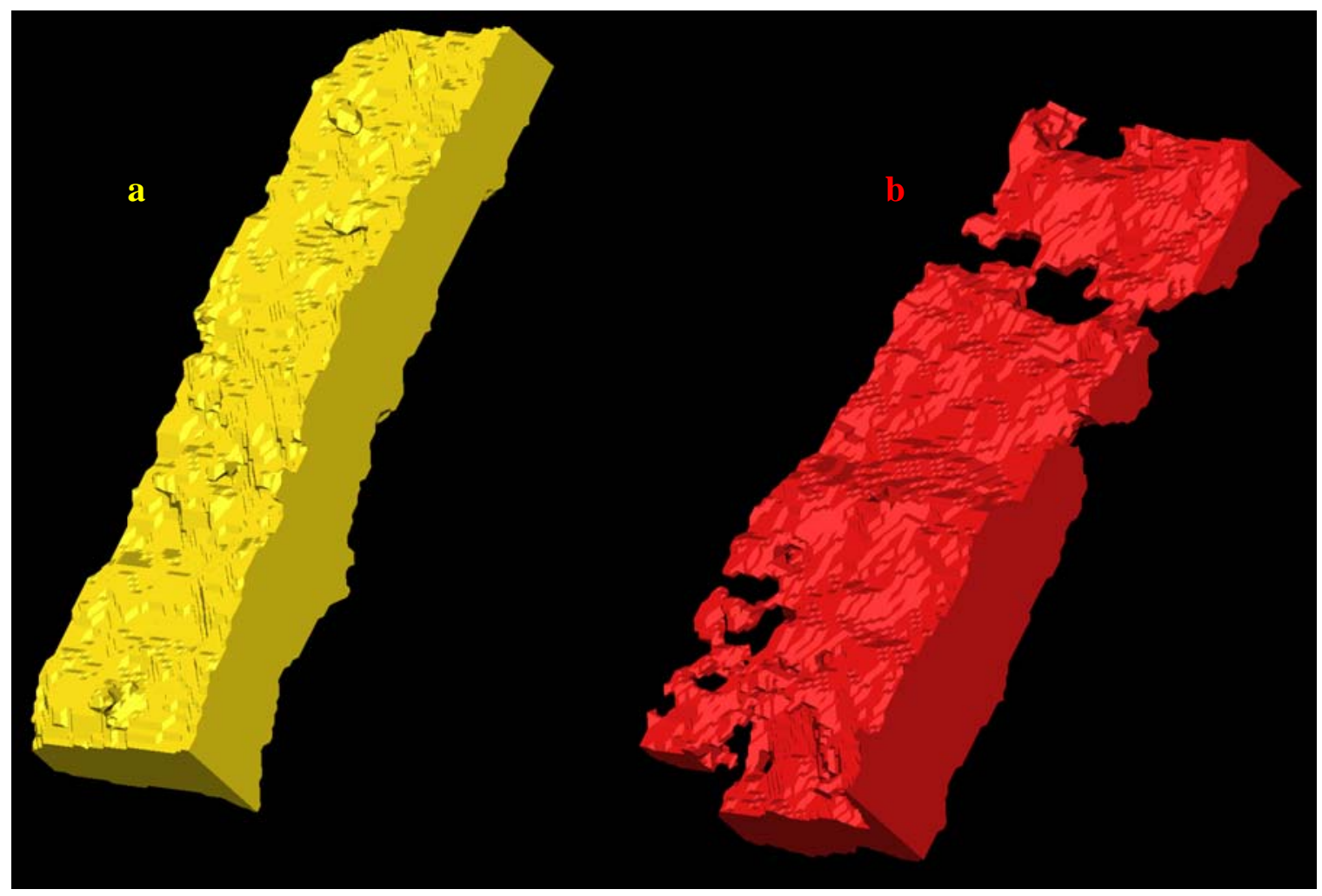

Figure 84: SMI distribution for the $16 \times 16$ case at 2500 psig. The width is

$3.120 \mathrm{~mm}$ and the length is $3.968 \mathrm{~mm}$.

So far, the effect of confining pressure has been addressed by using the Euler-Poincaré and the Structure Model Index. The EPC and SMI parameters were computed for the entire fracture. However, a segmentation process over the fracture intends to find the minimum representative volume in which the SMI and EPC are stable. Individual EPC and SMI values will help to find specific changes in the fracture that can correlate the fluid flow patterns in the fracture. Figure 85 shows the Euler-Poincare for the 4 cases, $2 \times 2,4 \times 4,8 \times 8$ and $16 \times 16$ where the average is slightly decreasing when the segmentation increases. The Euler-Poincaré value for the entire fracture was 0.4321 at 2500 psig. 
Poincare 2500 2x2

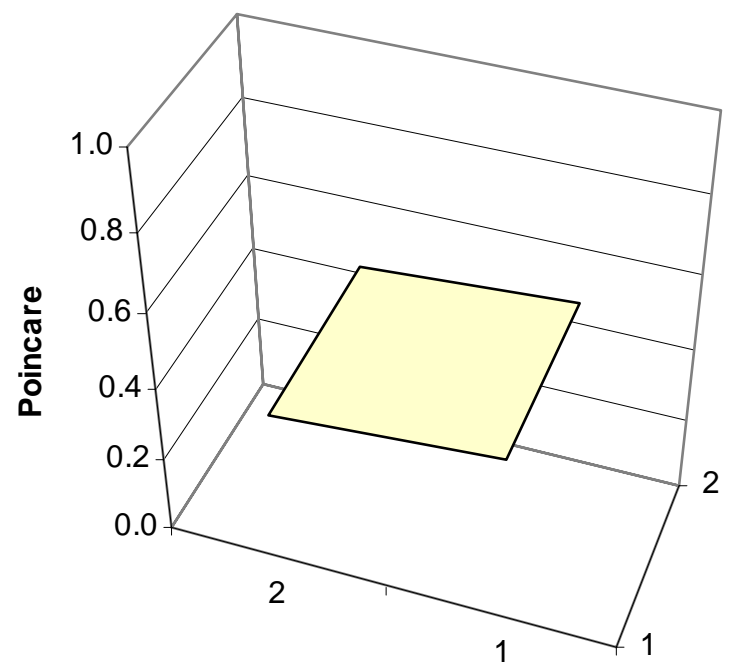

Average $=0.4314$

Poincare $25008 \times 8$

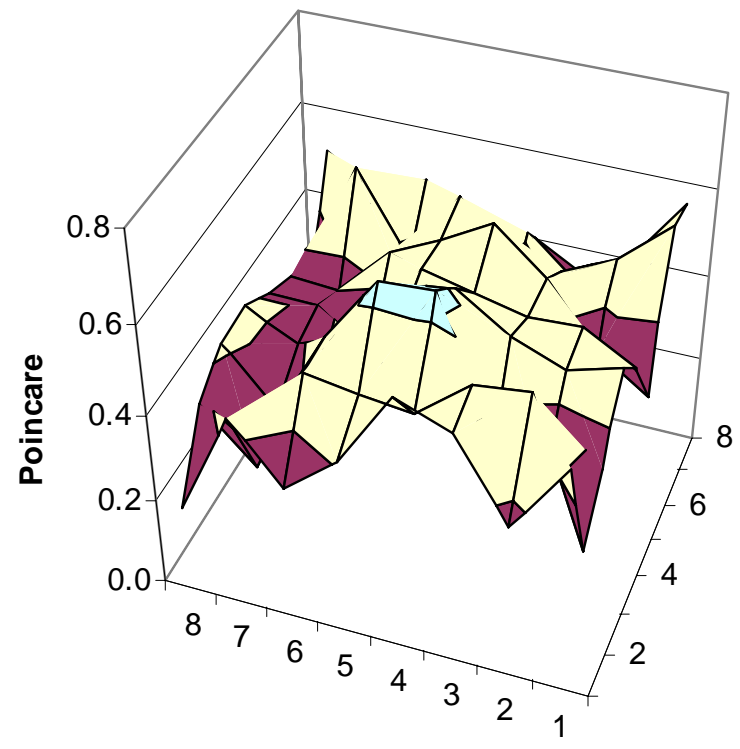

Average $=0.4295$
Poincare 2500 4x4

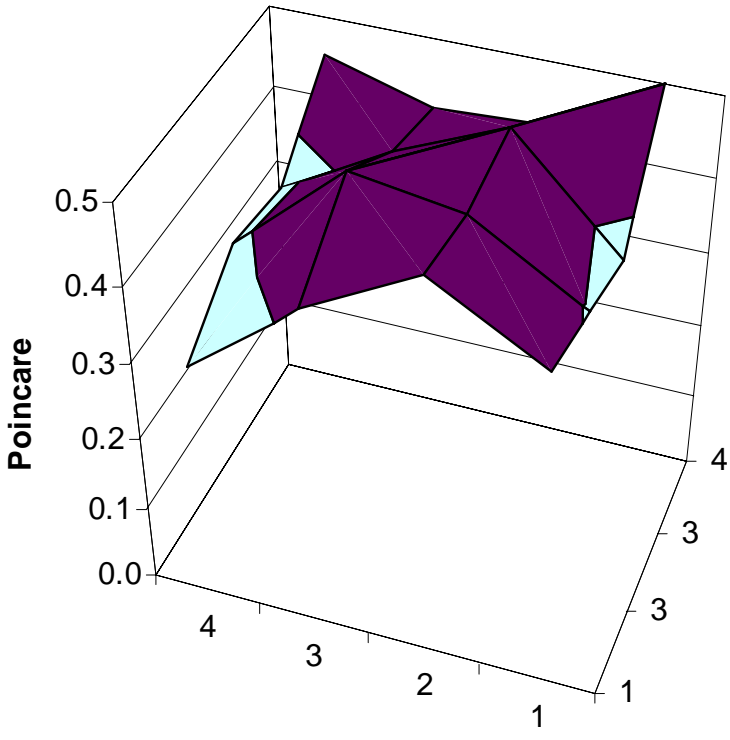

Average $=0.4306$

Poincare 500 16x16

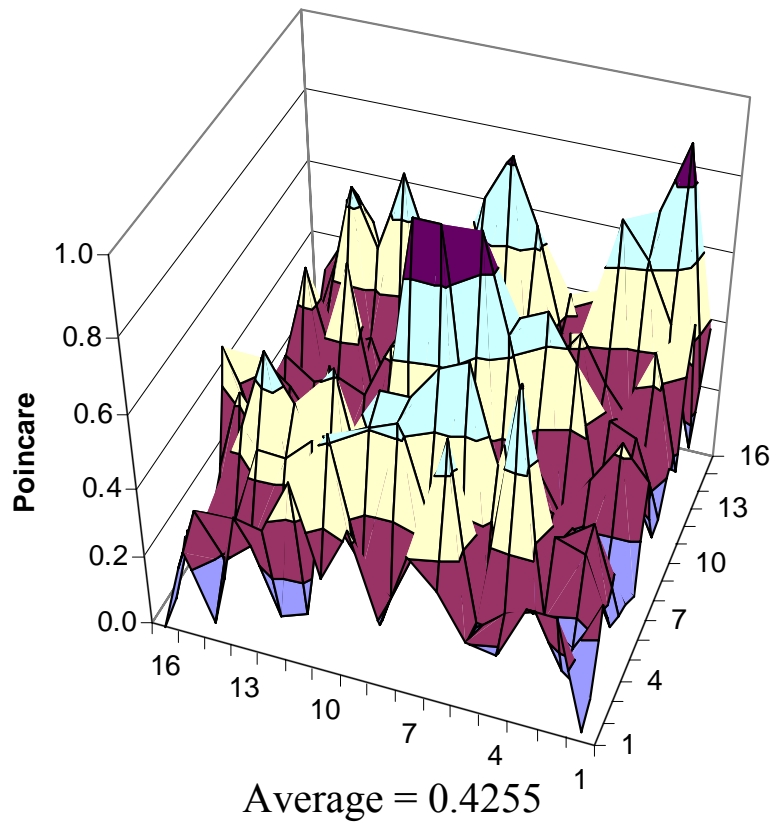

Figure 85: EPC distribution for the $2 \times 2,4 \times 4,8 \times 8$ and $16 \times 16$ case at 2500 psig. 
The average value for the Euler Poincaré does not significantly change for the given refining process, $2 \times 2,4 \times 4,8 \times 8$ or $16 \times 16$. Figure 86 show the average values for those grid blocks at 500 and 2500 psig.

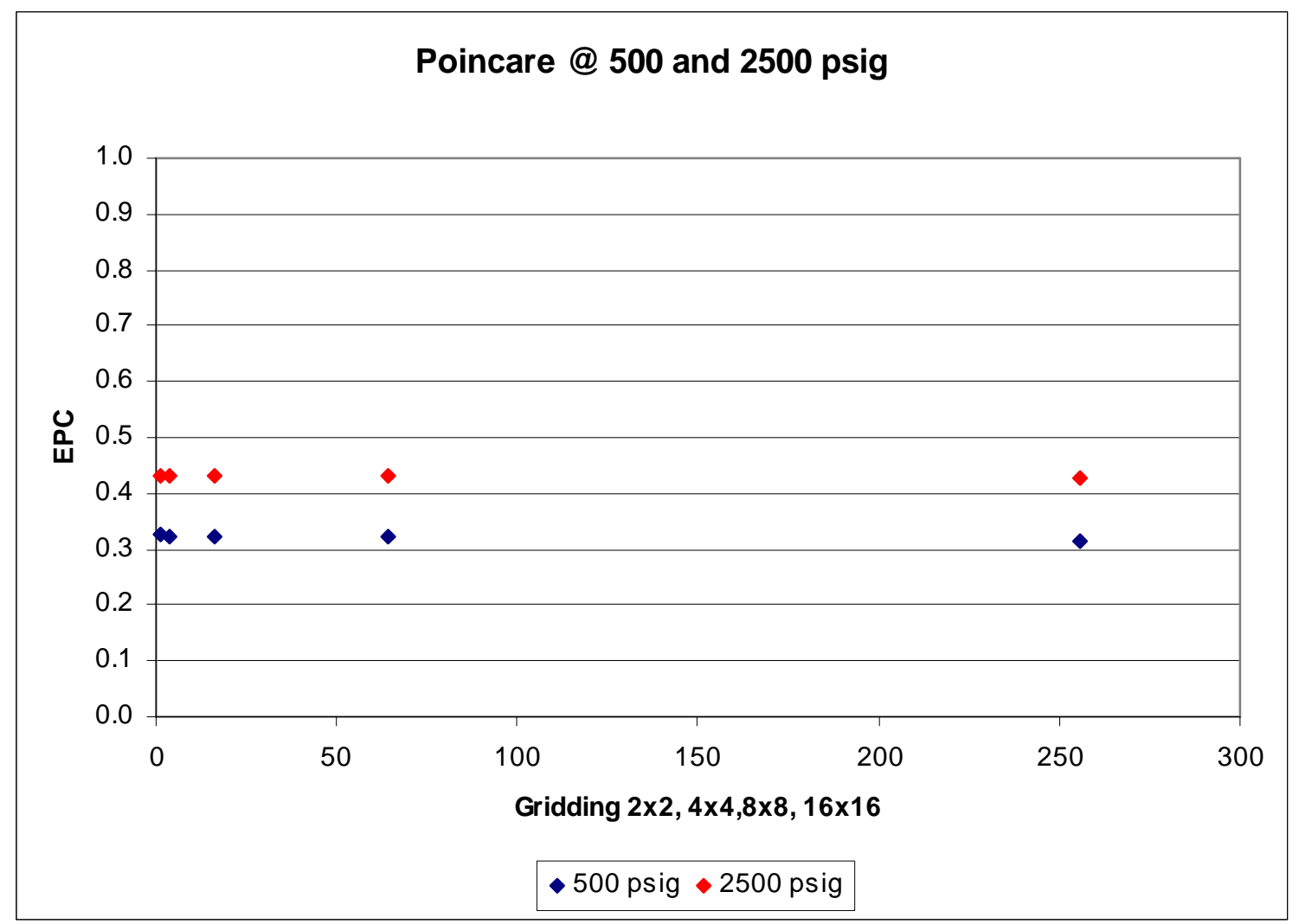

Figure 86: EPC average values for the $2 \times 2,4 \times 4,8 \times 8$ and $16 \times 16$ case at 500 and 2500 psig.

The SMI average values seem to be more sensitive to the segmentation process. Figure 87 shows the SMI behavior with respect to the segmentation process. 


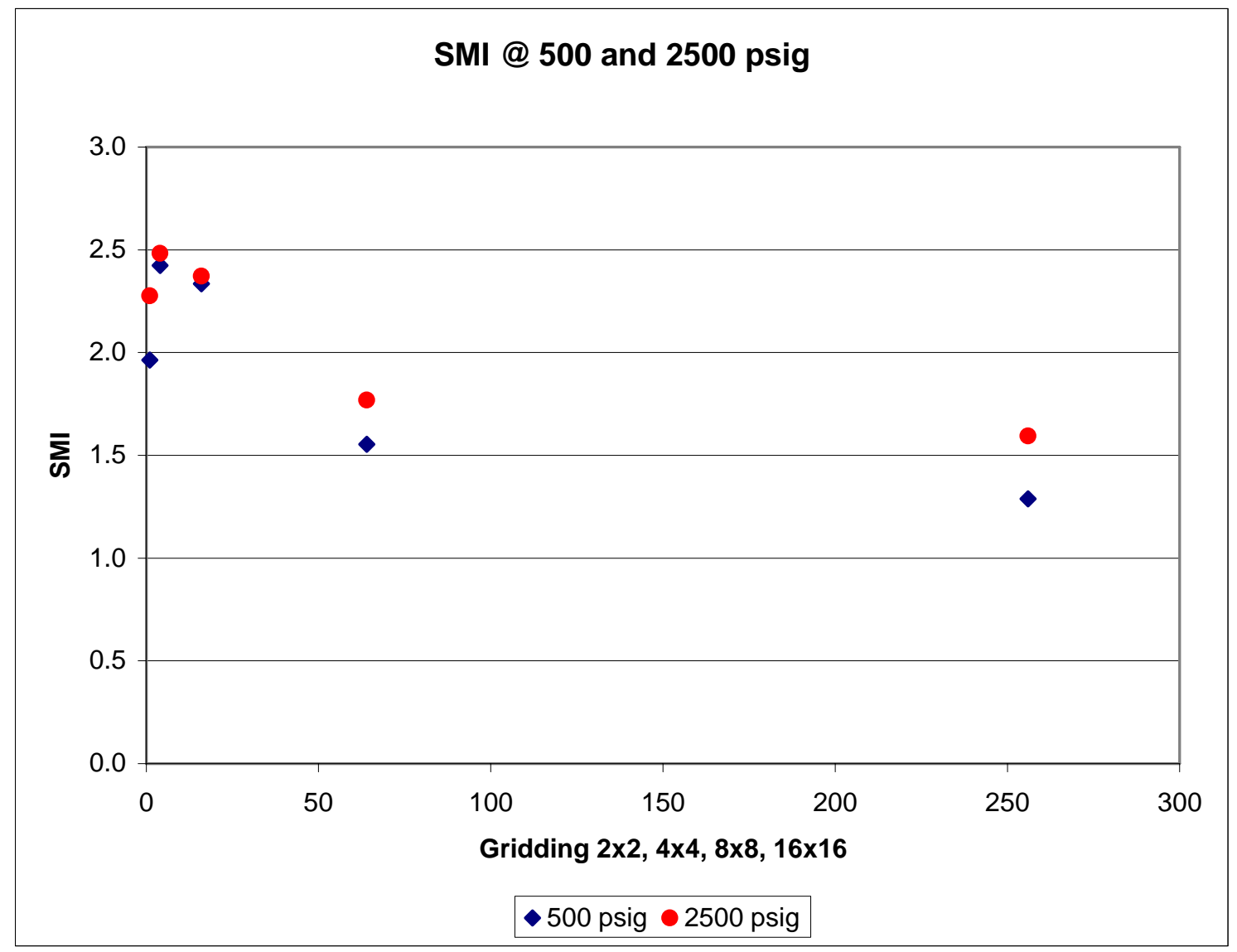

Figure 87: SMI average values for the $2 \times 2,4 \times 4,8 \times 8$ and $16 \times 16$ case at 500 and 2500 psig.

The SMI values of the confining pressure of 2500 are (red points) are higher than the ones computed from the 500 psig, highlighting a more rod type of structure. However, as the fracture is segmented, the SMI shows an increase in it values at the beginning of the segmentation process $(2 \times 2)$, followed by a monotonic decrees. Oscillations of the SMI average value would compromise the utilization of individual SMI for a general fracture characterization. 
Fluid flow experiments: All the flow experiments were performed using a Berea sandstone core. The measured absolute permeability, matrix flow, was on the order of $100 \mathrm{mD}$ (using water with $7 \%$ by weight $\mathrm{KCl}$ ). The cores were fractured as described in Chapter 4. After creating the tensile fracture, the two halves of the fracture were shifted axially by 200, 300 and 400 microns in order to create new asperities in the system. Different total liquid flow rate, 3, 6, 9 and $12 \mathrm{cc} / \mathrm{min}$ were tested. Based on the average fracture aperture and the pressure drop obtained, all cores were shifted by 400 microns and a total flow rate of $12 \mathrm{cc} / \mathrm{min}$ was used. Different fractional flow values were chosen to evaluate the effect of multiphase flow on rocks with single fractures where most of the flow is expected to be in the fracture.

The injection of the two phases (Brine and Kerosene) into the fracture was performed using the end plugs described previously. The pressure drop in the fracture was measured at the faces of the fracture using two pressure ports, one for each flow distributor. A highaccuracy digital pressure transducer with a range from 0-18 psig was used.

Table 11 shows the different flow rates for liquids and the fractional flow values used to create changes in the overall pressure drop of the system. The fractional flow is defined as the ratio of water flow rate divided by the total liquid flow rate, see Equation 3. 


$$
f w=\frac{q_{w}}{q_{w}+q_{o}}
$$

Where $\mathrm{q}_{\mathrm{w}}$ represents the water flow rate and $\mathrm{q}_{\mathrm{o}}$ the oil flow rate (kerosene).

The changes in pressure drop are a consequence of the difference in relative permeability, saturation history, and different viscosities and interfacial tensions.

Table 12: Liquid flow rates and fractional flow

\begin{tabular}{|c|c|c|c|}
\hline $\begin{array}{c}q_{w} \\
\text { (cc/min) }\end{array}$ & $\begin{array}{c}q_{o} \\
\text { (cc/min) }\end{array}$ & $\begin{array}{c}q_{I} \\
(c c / m i n)\end{array}$ & $f w$ \\
\hline 12.0 & 0.0 & 12 & 1.00 \\
\hline 11.0 & 1.0 & 12 & 0.92 \\
\hline 10.0 & 2.0 & 12 & 0.83 \\
\hline 9.0 & 3.0 & 12 & 0.75 \\
\hline 8.0 & 4.0 & 12 & 0.67 \\
\hline 7.0 & 5.0 & 12 & 0.58 \\
\hline 6.0 & 6.0 & 12 & 0.50 \\
\hline 5.0 & 7.0 & 12 & 0.42 \\
\hline 4.0 & 8.0 & 12 & 0.33 \\
\hline 3.0 & 9.0 & 12 & 0.25 \\
\hline 2.0 & 10.0 & 12 & 0.17 \\
\hline 1.0 & 11.0 & 12 & 0.08 \\
\hline 0.0 & 12.0 & 12 & 0.00 \\
\hline
\end{tabular}


Core \#1: Figure $\mathbf{8 8}$ presents the pressure drop along the fracture after changing the confining pressure from 800 to $1000 \mathrm{psig}$ at the fixed injection rate. The pressure drop in the system at 1000 psig shows a higher value than in $800 \mathrm{psig}$. This is a consequence of the closure of the fracture due to the increase in confining pressure. This behavior (aperture closure) with increase in confining pressure was mechanically confirmed in the previous section by using CT.

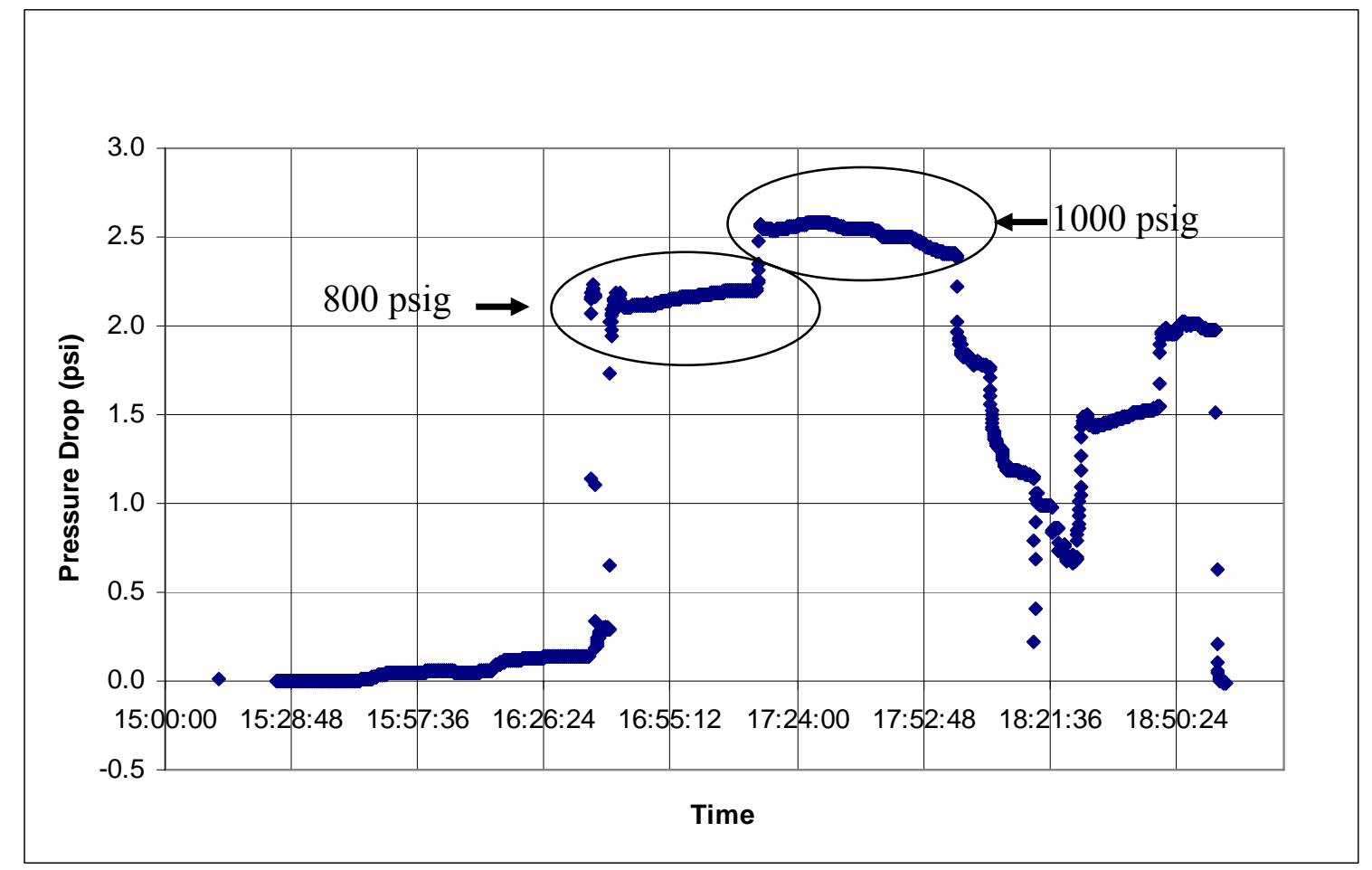

Figure 88: Changes along pressure drop for different confining pressure at fixed injection rate. 
Figure 89 shows the changes in the pressure drop (confining pressure of $800 \mathrm{psig}$ ) in the fracture for different fractional flows, from 1 to 0 . A global maximum value of 2.2 psi in the system is observed for a fractional flow of $33 \%$. A similar graph for a confining pressure of 1000 psig is shown in Figure 90 with a maximum pressure drop for a fractional flow of 0.4 .

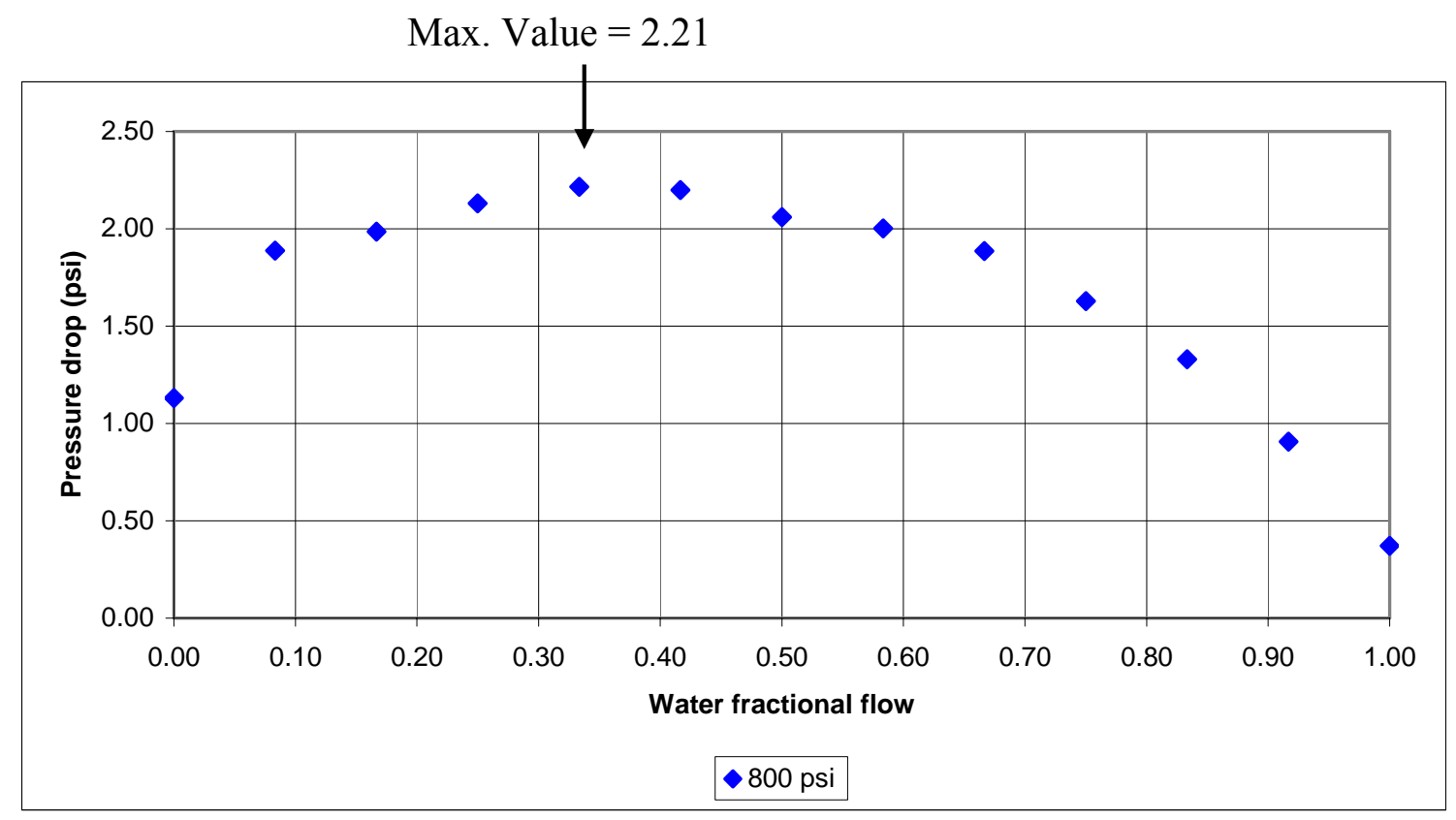

Figure 89: Pressure drop along the fracture for different fractional flow values. 
Max. Value $=2.59$

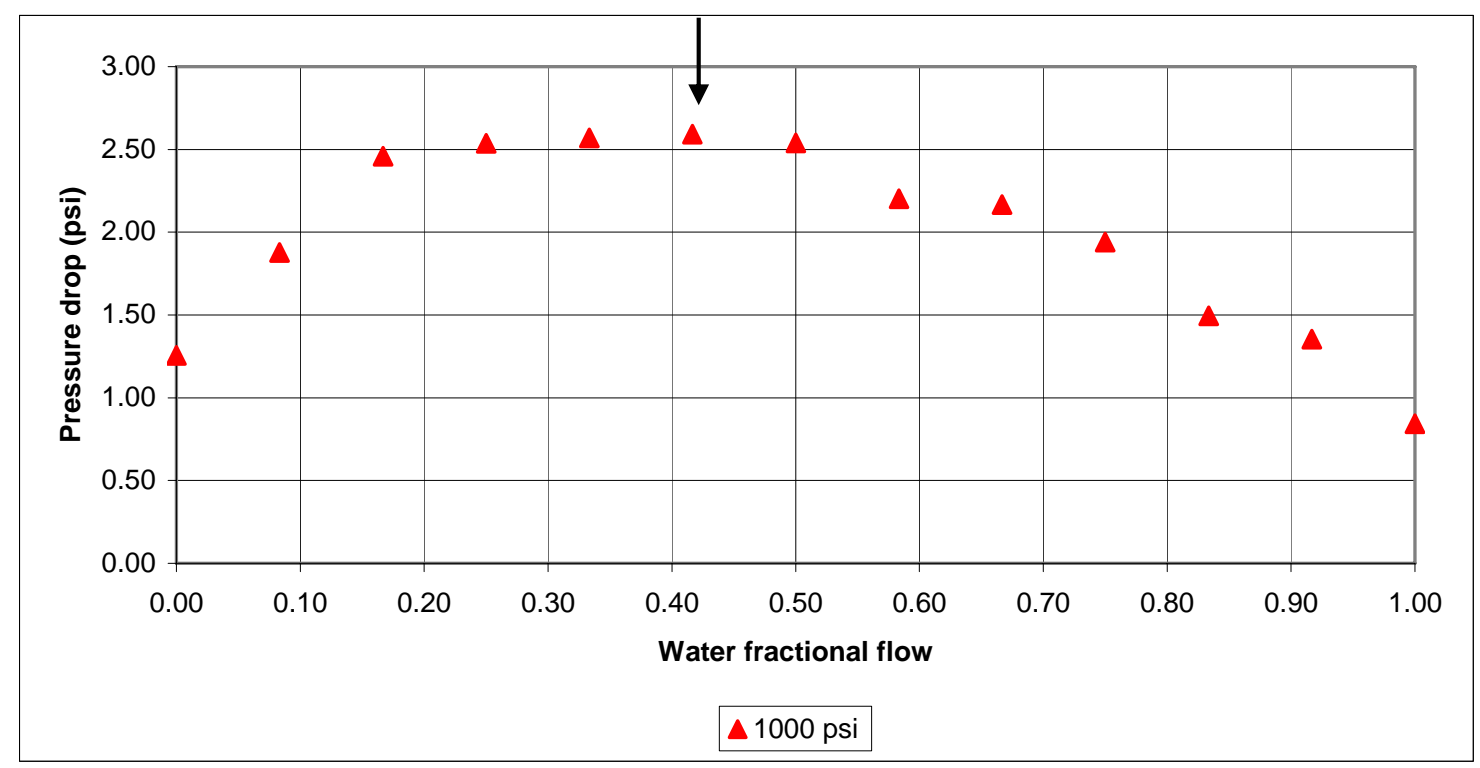

Figure 90: Pressure drop along the fracture vs fractional flow, 1000 psig confining pressure.

Figure 91 shows two pressure drop curves for two different confining pressures, 800 and 1000 psig. The difference between the two curves (Y axis) is due to the closure of the fracture aperture. For the two cases, 800 psig and 1000 psig confining pressure, a minimum pressure drop is observed for a fractional flow of 1.0 The fact that in both cases a higher pressure drop in observed for a $\mathrm{fw}=0.0$ than for $\mathrm{fw}=1.0$ would probably corresponds not only the viscosity ratios but also to the wettability of the system, the water being the wetting phase in this experiment. 


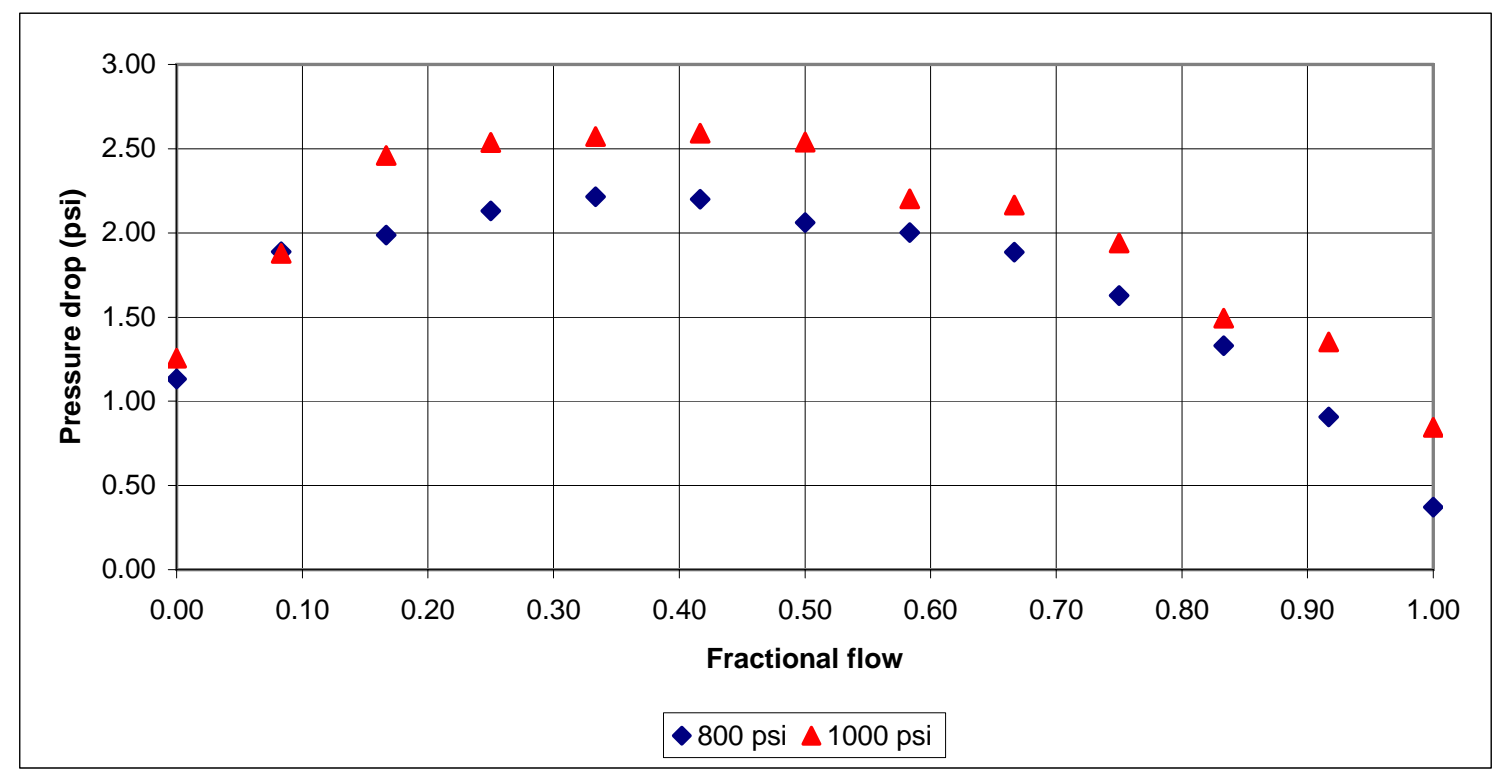

Figure 91: Pressure drop in the fracture vs fractional flow for 800 and 1000 psig.

Core \#q: To analyze the fracture topology (variability) effects, a second Berea Sandstone core was used for a fluid injection experiments. Different fractional flow conditions were applied from 0.0 to 1.0 and from 1.0 to 0.0 . Figure 92 shows saturation dependency on the flow paths. At fractional flow lower than 0.5 , hysteresis seems to be less than at high fractional flow values. Figure $\mathbf{9 3}$ presents a second set of fractional flow tests for the same rock sample, showing a consistency (repeatability) in the flow paths. 


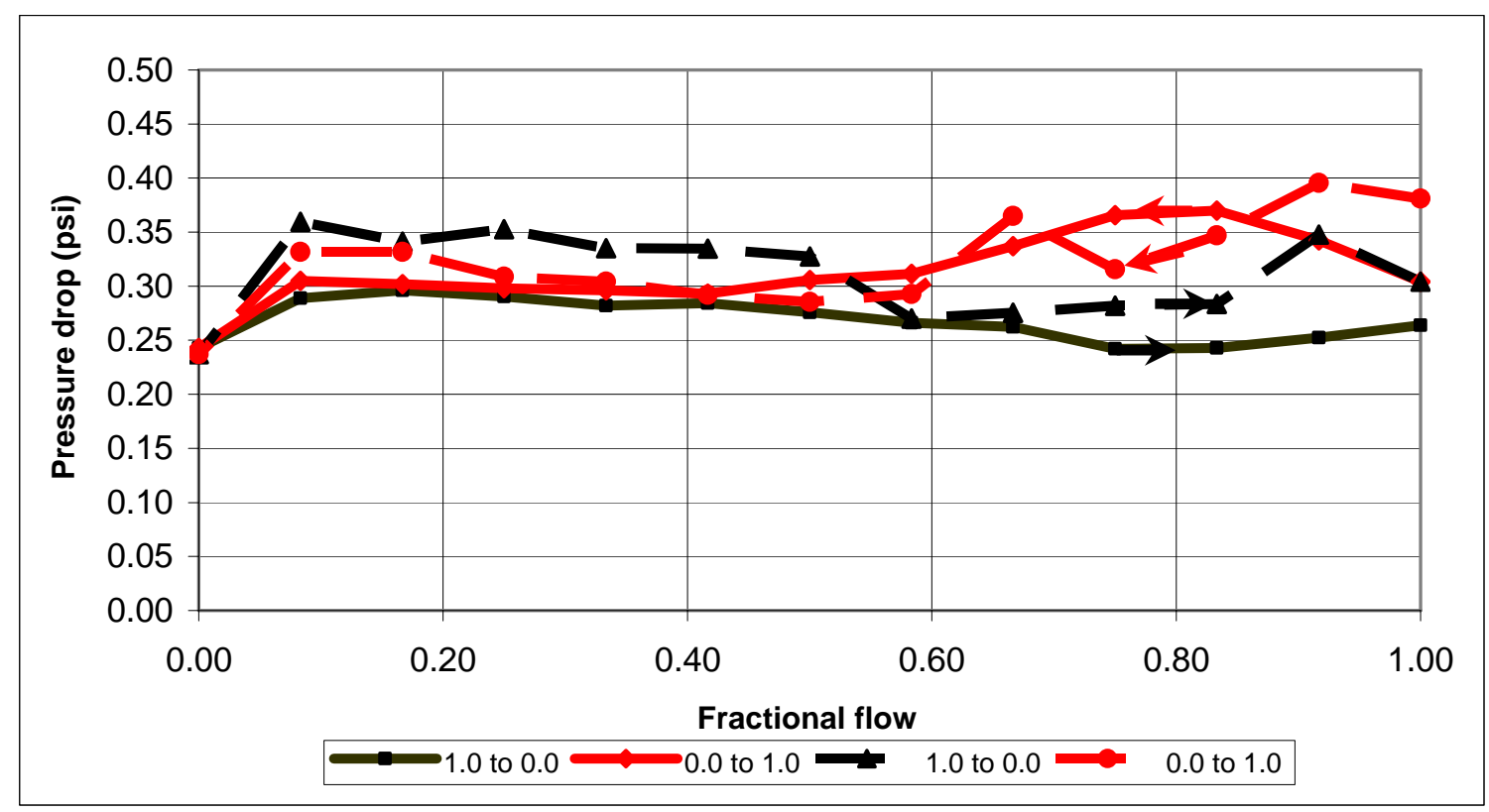

Figure 92: Pressure drop in the fracture vs fractional flow for 200 psig. Set \# 1.

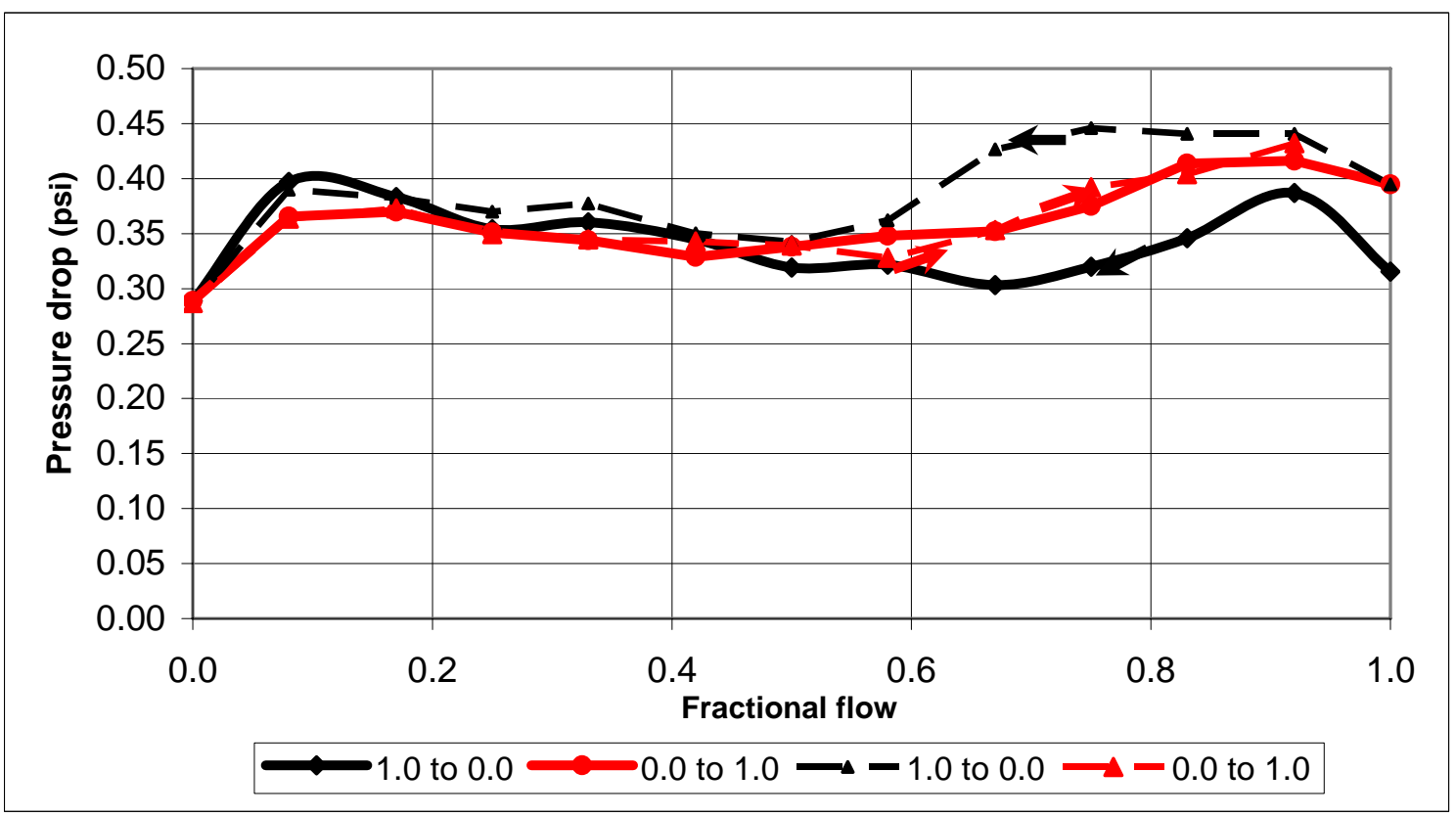

Figure 93: Pressure drop in the fracture vs fractional flow for 200 psig. Set \# 2. 
In order to maintain two phase flow injection into the fracture, the end points were not taken into consideration. The fractional flow changes were done after injecting $120 \mathrm{cc}$ (10 $\min )$. However, in some cases the volume was higher $(240 \mathrm{cc})$ based on the equilibrium of the pressure drop in the system.

Core \#3: Figure 94 shows the fractional flow behavior for another Berea Sandstone core. The pressure drop along the core presents a different shape than previous experiment. Figure 5.35 also highlights a critical point, at fractional flow equal to 0.5 , where the pressure drop behavior increases as the fractional flow increases.

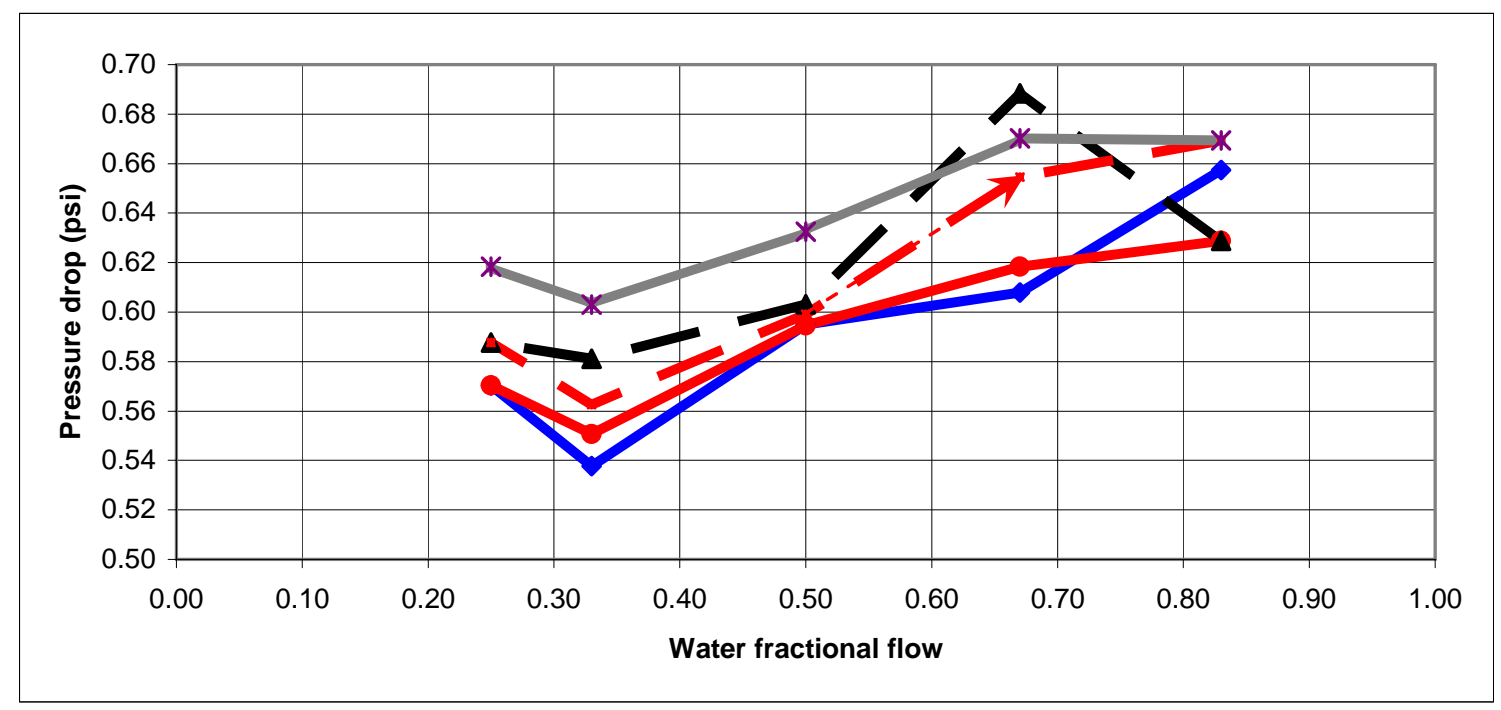

Figure 94: Pressure drop in the fracture vs fractional flow for a confining pressure equal to 800 psig. 
Figure 95 shows the pressure drop in the fracture for the section of fractional flow from 0.25 to 0.5 . A very repeatable behavior is observed for this section. The section of fractional flow from 0.5 to 0.83 presents a non repeatable behavior shown in Figure 96 .

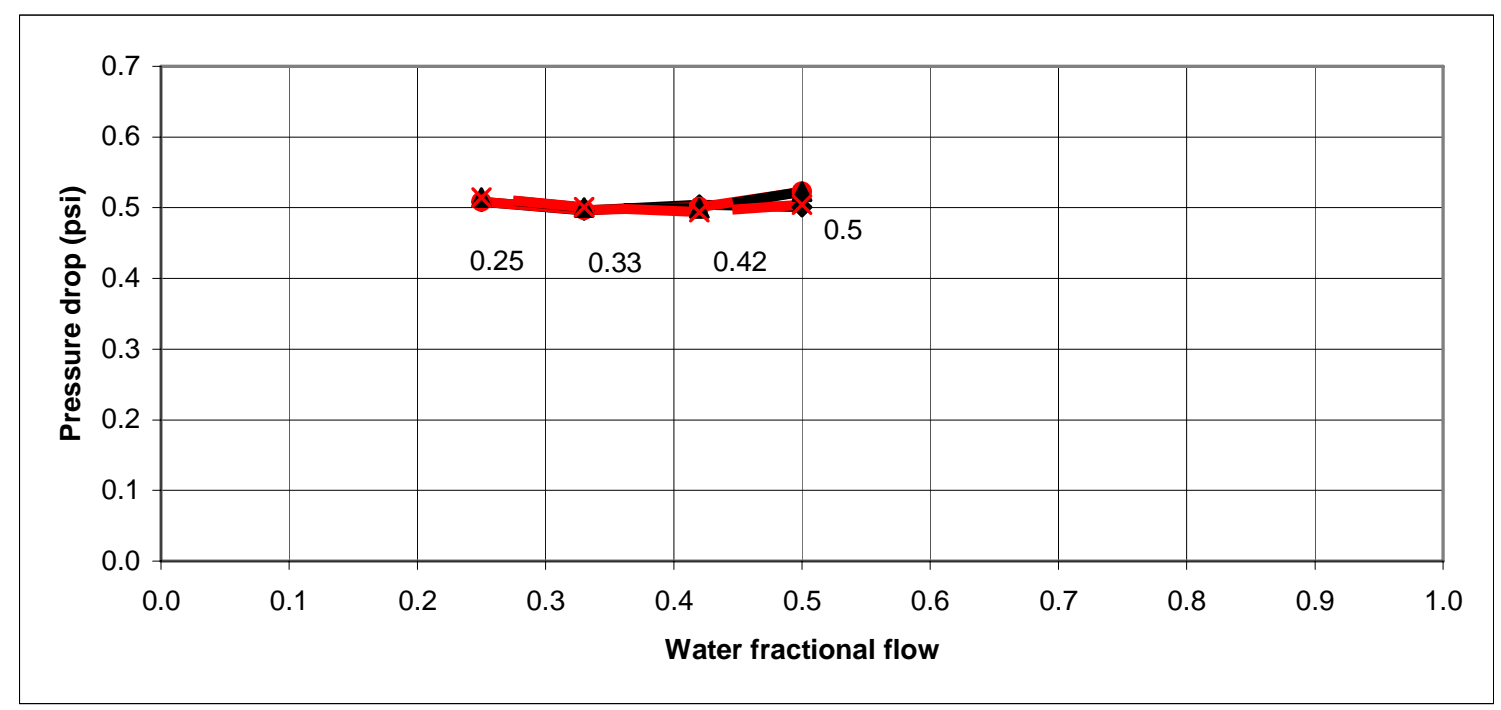

Figure 95: Fractional flow range: $0.25-0.50 .800$ psig confining pressure.

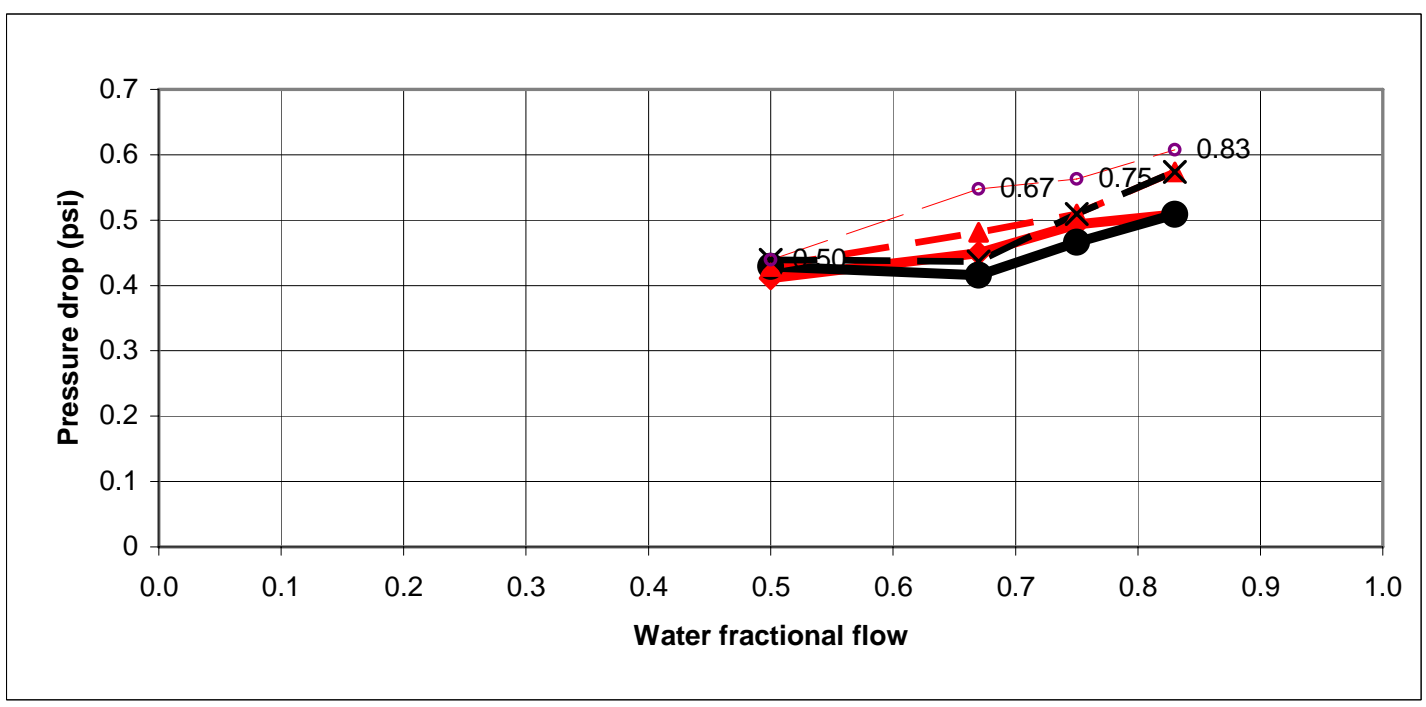

Figure 96: Fractional flow range: $0.50-0.83 .800$ psig confining pressure. 
Core \#4: The core (4) was used for fluid flow shows different flow behavior under 500 psig confining pressure. With the increase in the fractional flow of water the pressure drop decreases. The confining pressure for this experiment is different from previous experiments (500 psig). Figure 97 shows the pressure drop in the fracture for different fractional flow at 500 psig confining pressure. The fracture topology for this core seems to be different from other cores as a consequence of the change in confining pressure, having an important effect on the flow patterns. So far, the flow analyses have been performed with no $\mathrm{CT}$ information. In future experiments $\mathrm{CT}$ that will be acquired to create saturation maps that will lead to explain the observations and hypotheses pretended in this study.

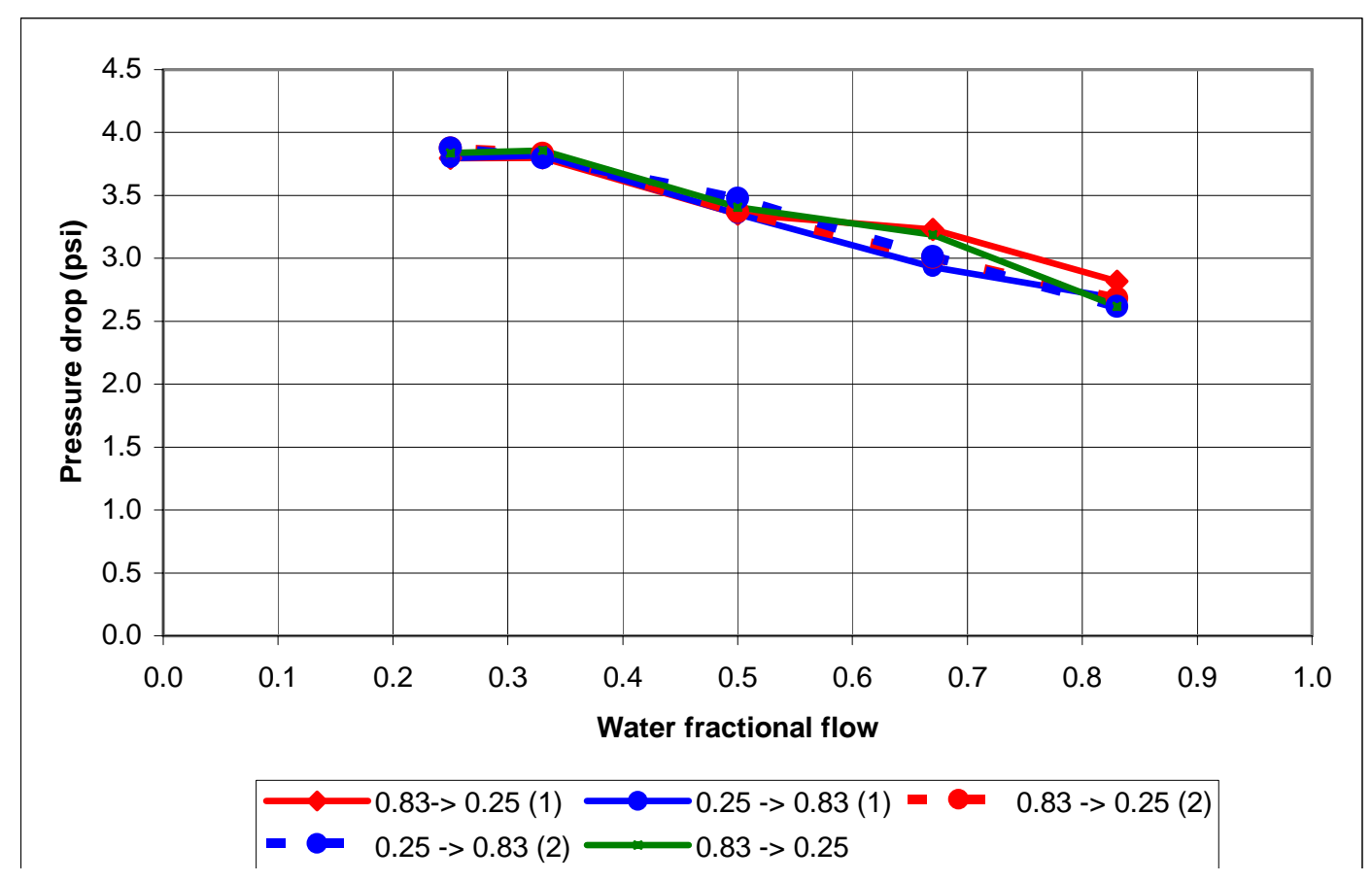

Figure 97: Pressure drop in the fracture vs fractional flow for 500 psig. Core \# 4. 
Multi-Phase flow injection with CT data: A Berea sandstone core with a $2.5 \mathrm{~cm}$ diameter and $7 \mathrm{~cm}$ in length was used, where the bedding planes were axial and perpendicular to the fracture. Figure 98 (top) presents the final cropping for the matrix and fracture. A second reduction in size is necessary, given the computational memory need to analyze and manipulate the data. Figure 98 (bottom) shows the reduction to a rectangle of $\mathrm{x}=22.1, \mathrm{y}=3.12$ and $\mathrm{x}=68.3 \mathrm{~mm}$ in size.

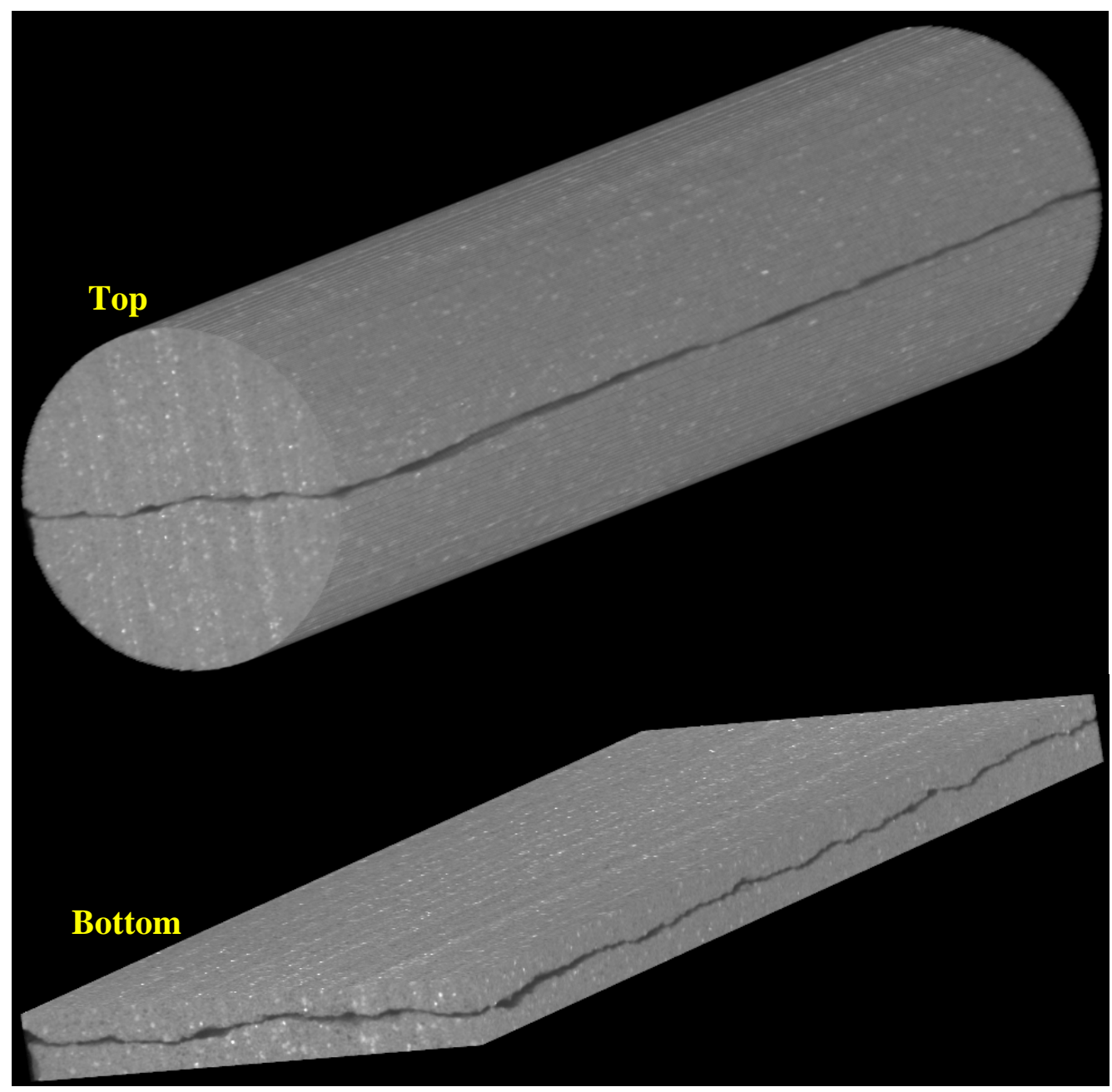

Figure 98: Fractured core (top), matrix reduction (bottom). 
After vacuum-saturating the sample the absolute permeability to water was measured. Water was injected at different flow rates. Figure $\mathbf{9 9}$ shows the pressure drop along the fracture as a function of water injection rates.

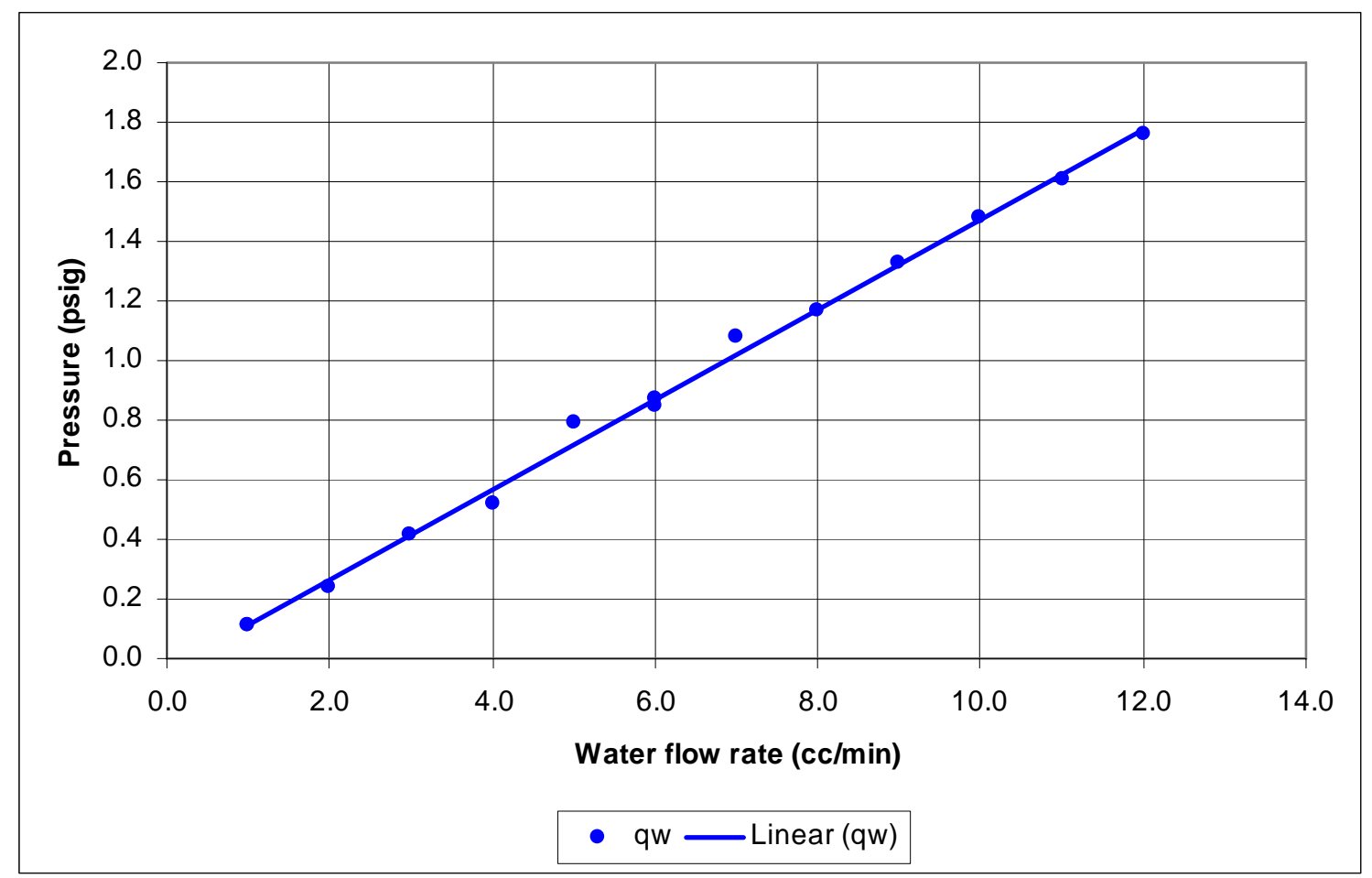

Figure 99: Pressure drop in the fracture for water injection.

A comparison of the viscosities of water and oil showed similarities in the order of magnitude for both fluids. Oil viscosity was $0.953 \mathrm{cP}$ and water viscosity was $1.006 \mathrm{cP}$. 
The parallel plate approximation, Witherspoon et al. (1980) has been used to determine the pressure drop along fracture during single phase flow. The cubic law equation is:

$Q=\frac{\Delta p}{12 \mu L} b^{3} W$

where $\mu$ is the dynamic viscosity of the fluid $\left[\mathrm{ML}^{-1} \mathrm{~T}^{-1}\right], \mathrm{L}$ is length of the sample $[\mathrm{L}], \mathrm{W}$ is the diameter of the sample $[\mathrm{L}], \mathrm{Q}$ is the flow rate $\left[\mathrm{L}^{3} \mathrm{~T}^{-1}\right], \Delta \mathrm{p}$ is the differential pressure $\left[\mathrm{ML}^{-2} \mathrm{~T}^{-1}\right]$, and $b[\mathrm{~L}]$ is the equivalent hydraulic aperture of the fracture.

Equation 4 was used to predict the pressure drop along the fracture using the fracture average aperture obtained by CT data $(0.264 \mathrm{~mm})$. The computed pressure drop for a flow rate of $12 \mathrm{cc} / \mathrm{min}$ was equal to $0.0622 \mathrm{psi}$. The pressure drop along the fracture measured experimentally was 2.31 psi. The considerable difference between the estimated cubic law and the measured value (ratio of 37) is due the complicated topology of the fracture. 
Figure 100 highlights the pressure drop in the fracture for different flow conditions. The $100 \%$ water flow is shown by the blue circles. Oil was injected into the sample driving the water saturation to its residual value, $\mathrm{S}_{\text {wirr. }}$ At this condition a set of measurements were made shown by the red diamonds. Kerosene viscosity is close to water viscosity $\left(\mu_{\mathrm{wNai}}=1.006 \mathrm{cP}, \mu_{\mathrm{Kero}}=0.953 \mathrm{cP}\right)$ resulting in a similar response to the $100 \%$ watersaturated sample with a $k_{\mathrm{ro}}\left(\mathrm{S}_{\mathrm{wir}}\right)=0.62$. The water flow at residual oil saturation (green triangles) shows a reduction in water permeability, where $\mathrm{k}_{\mathrm{rw}}\left(\mathrm{S}_{\mathrm{or}}\right)=0.306$.

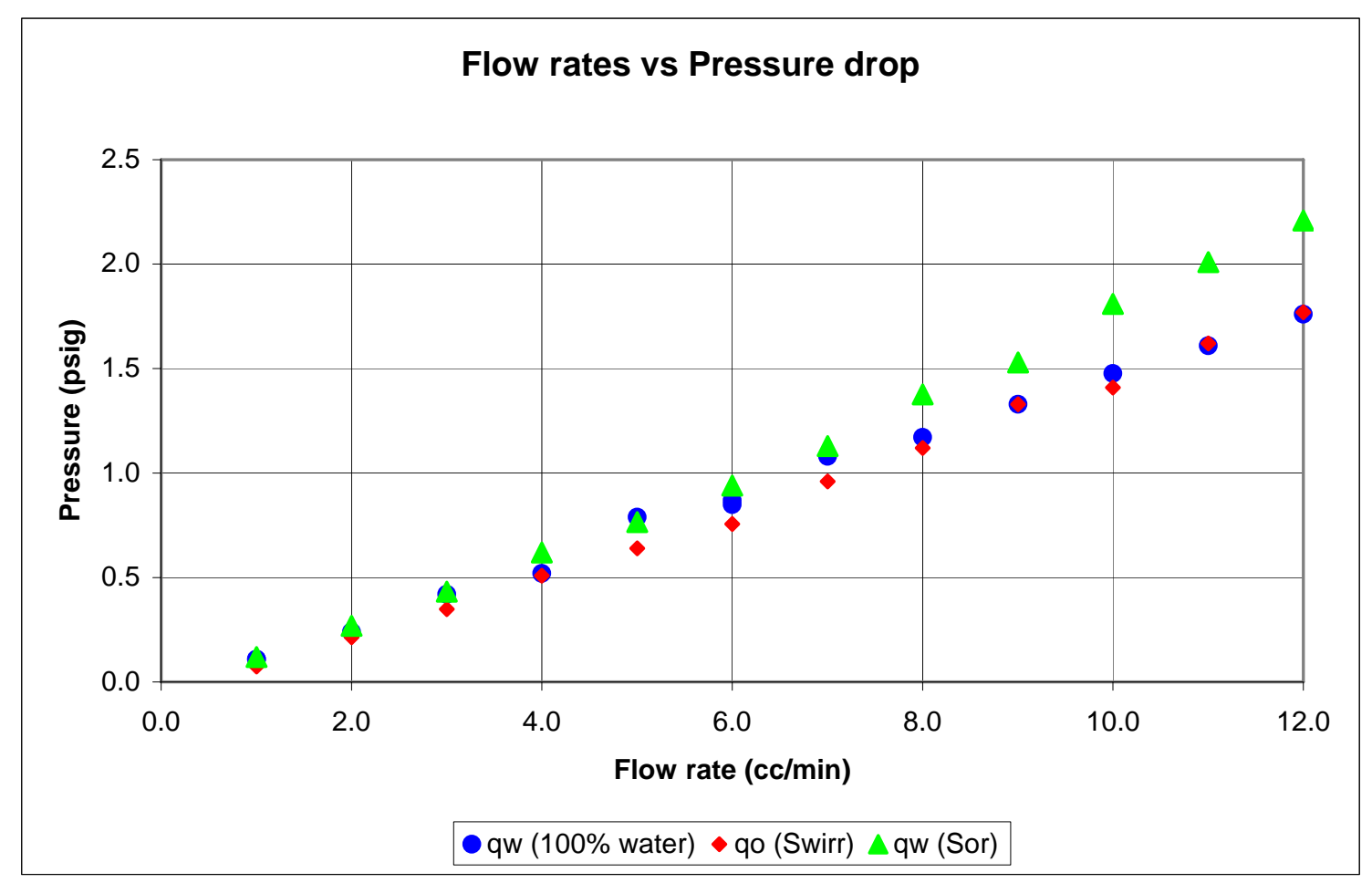

Figure 100: Water pressure drop in the fracture for different saturation conditions. 
The relative permeabilities for the oil and water at $\mathrm{S}_{\mathrm{wirr}}$ and $\mathrm{S}_{\text {or }}$ and of an intermediate point, were the core was under two phase flow $\left(f_{w}=0.66\right)$ are shown in Figure 101 shows.

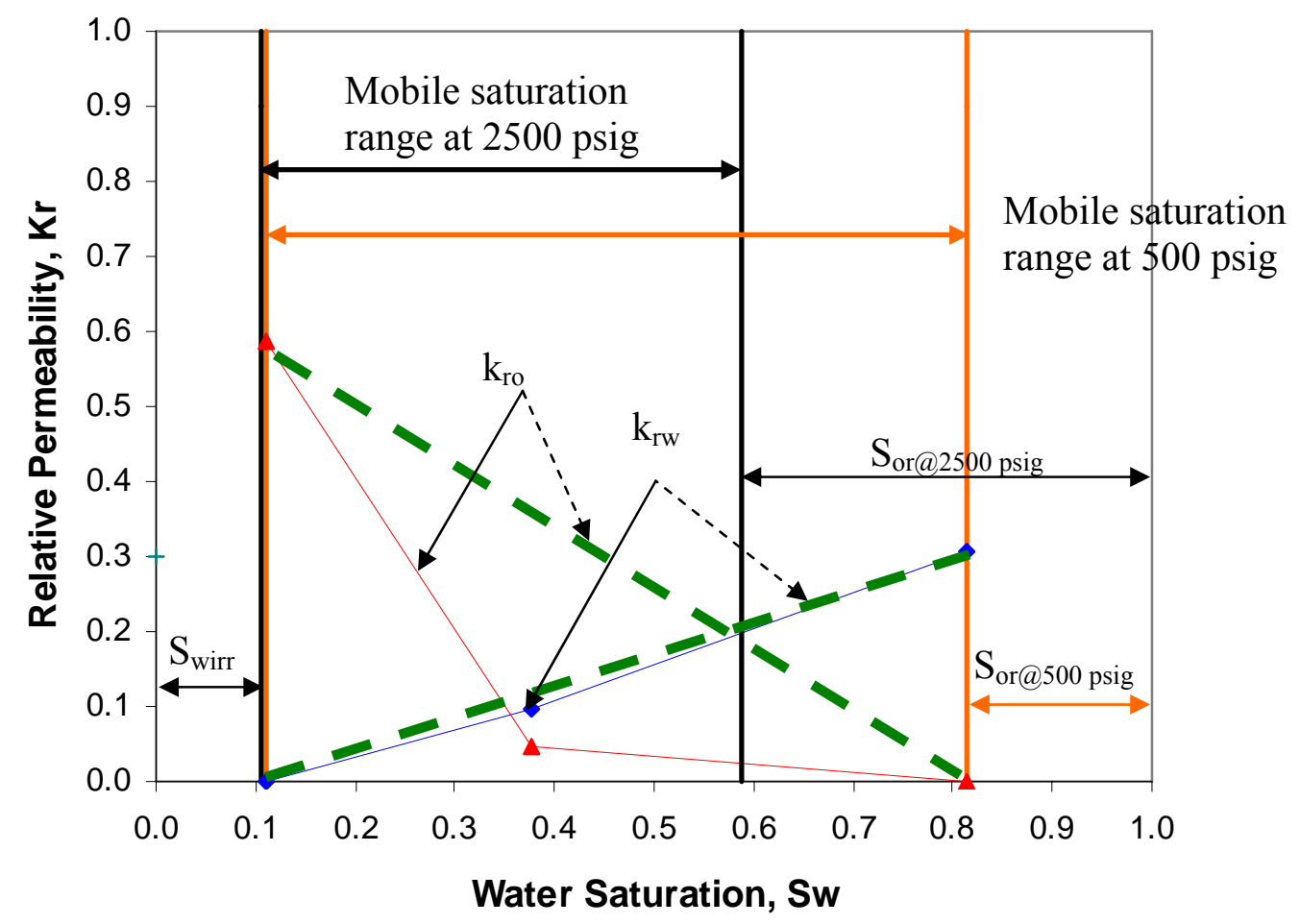

Figure 101: Relative permeabilities curve for a fractured system at 500 psig.

The green dash lines represent the $\mathrm{x}$-shaped curves for relative permeabilities for oil and water, usually used for fractured systems. The blue and red lines represent the experimental relative permeability, for water and oil respectively. A significant deviation from the straight line is observed for the $\mathrm{k}_{\mathrm{ro}}$ curve. The $\mathrm{x}$-shaped curves over estimate the permeability of the non-wetting phase. The mobile range at this low press (500 psig) is about $70 \%$. At 2500 confining pressure the mobile range saturation range was reduced to $48 \%$. 
To characterize and quantify the fracture volume a segmentation process is required. Figure 102 shows the changes of the fracture volume for different thresholds. For the dry fracture a threshold of 2400 was selected for a fracture volume of $0.376 \mathrm{cc}$ (at $500 \mathrm{psig}$ ) where the change in volume with threshold is not highly stiff.

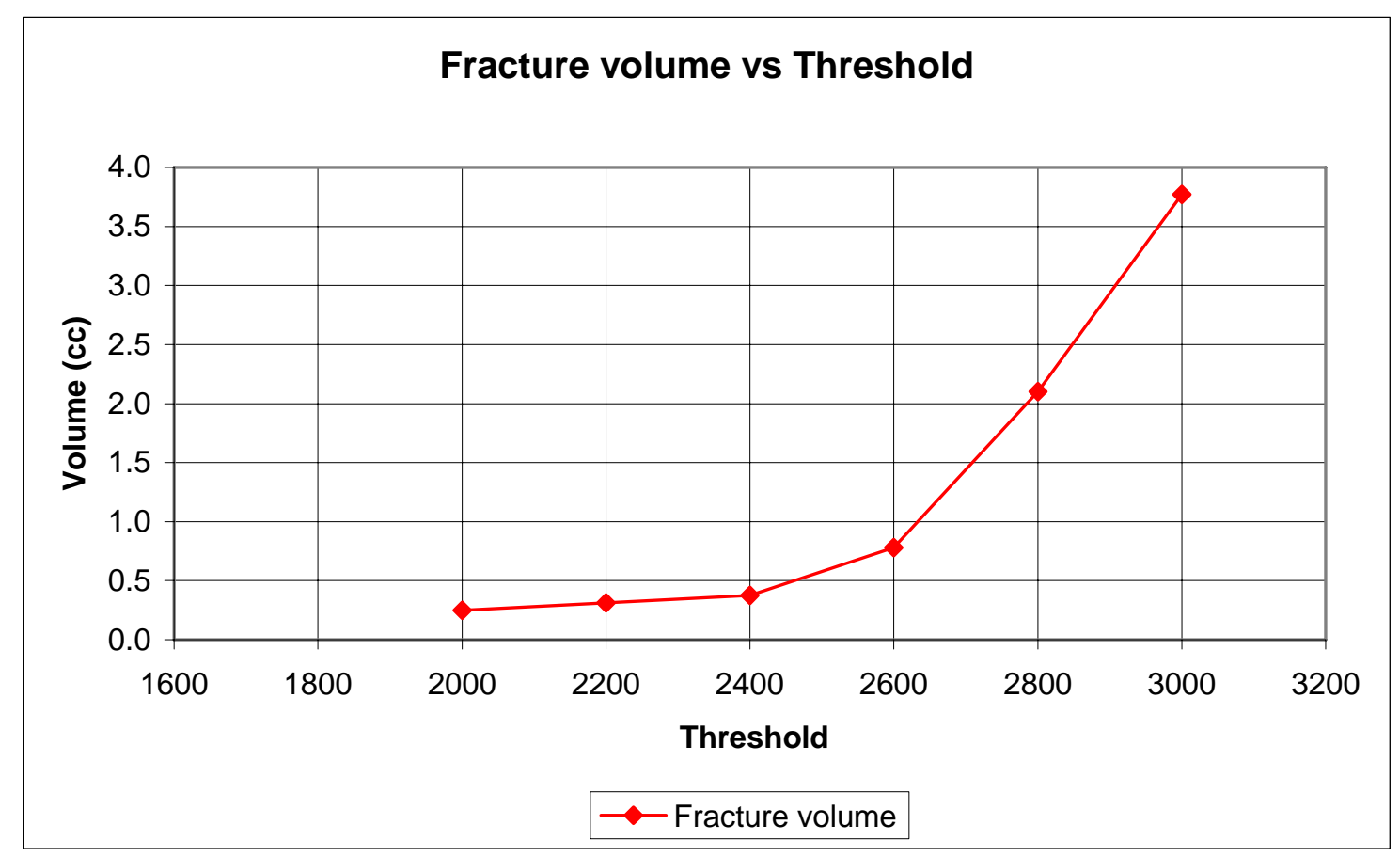

Figure 102: Fracture volume for different threshold numbers.

Figure 103 shows the results for the three dimensional fracture aperture with a fracture volume of $375.8 \mathrm{~mm}^{3}$. The black area represents the asperities (contact joints) in the system. 


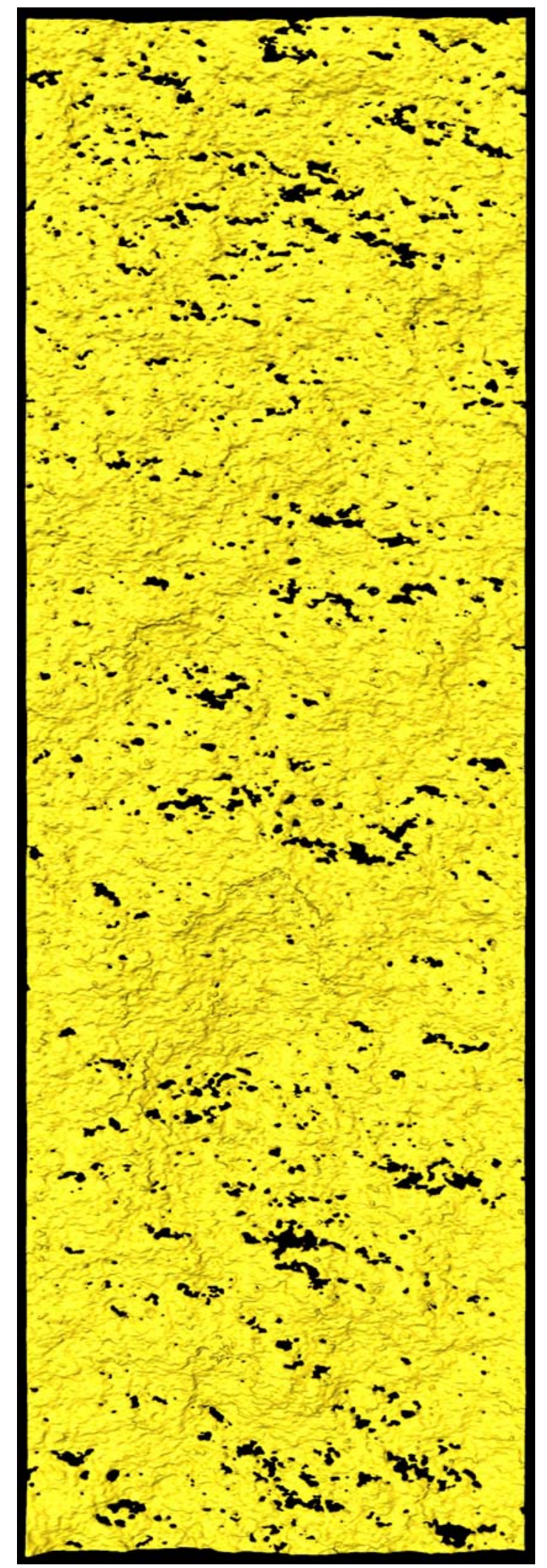

Figure 103: Three dimensional rendition of fracture asperities. 
Figure 104 shows the fracture at residual water saturation, where the oil occupies most of the fracture volume. From left to right, dry fracture, oil phase and water phase are presented. The computed fracture residual water saturation $\left(\mathrm{S}_{\mathrm{wirr}, \mathrm{f}}\right)$ was $11 \%$.

a

b

c

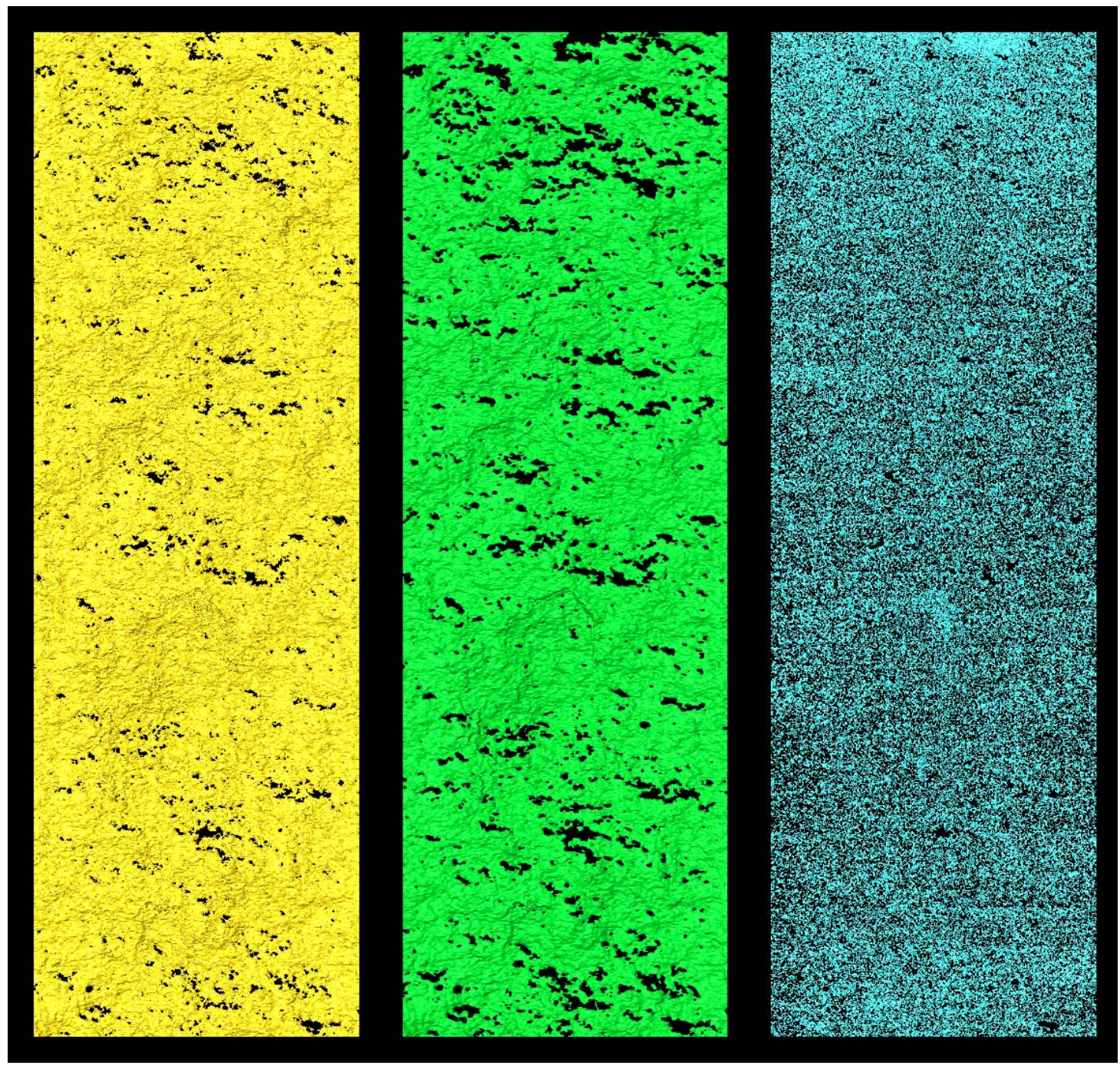

Figure 104: Fluids distribution maps: a: dry. b: oil. c: water at residual water saturation state. 
After reaching and scanning the system at $S_{\text {wirr }}$ the core was re-flooded with water to reach the residual oil saturation. Hundreds of fracture pore volumes were injected. At residual oil saturation the fluid saturations were computed: The corresponding saturations were $\mathrm{S}_{\mathrm{or}}=0.186$ and $\mathrm{S}_{\mathrm{w}}=0.814$. Figure 105 shows three dimensional rendition of the fracture. Fracture at dry condition (left), at residual oil (center) and the water (right) distribution. The oil is in discontinues isolated ganglias, and the water is the continuous phase.

a

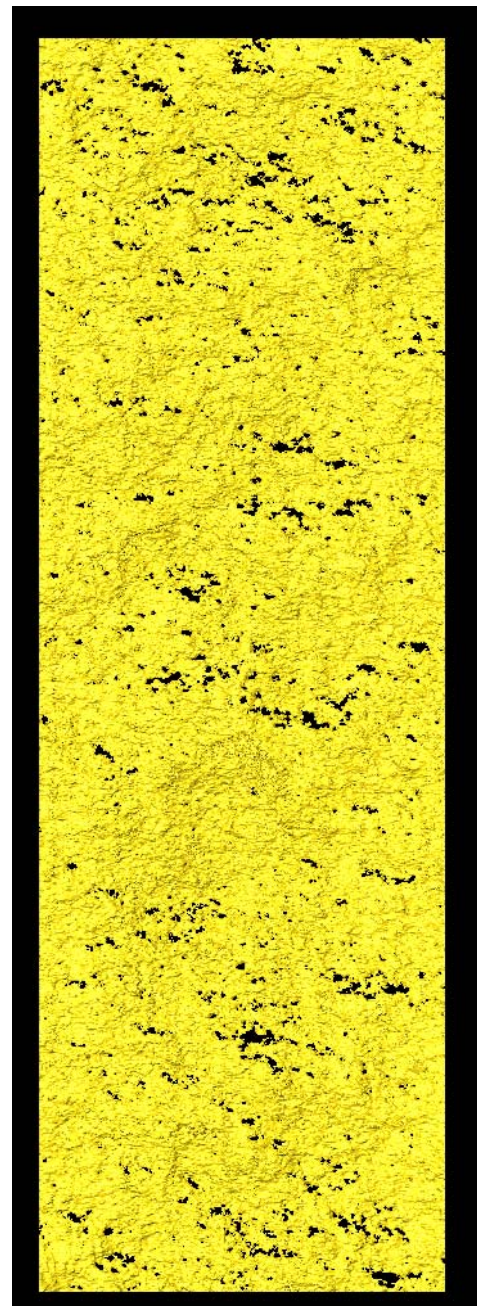

$\mathrm{b}$

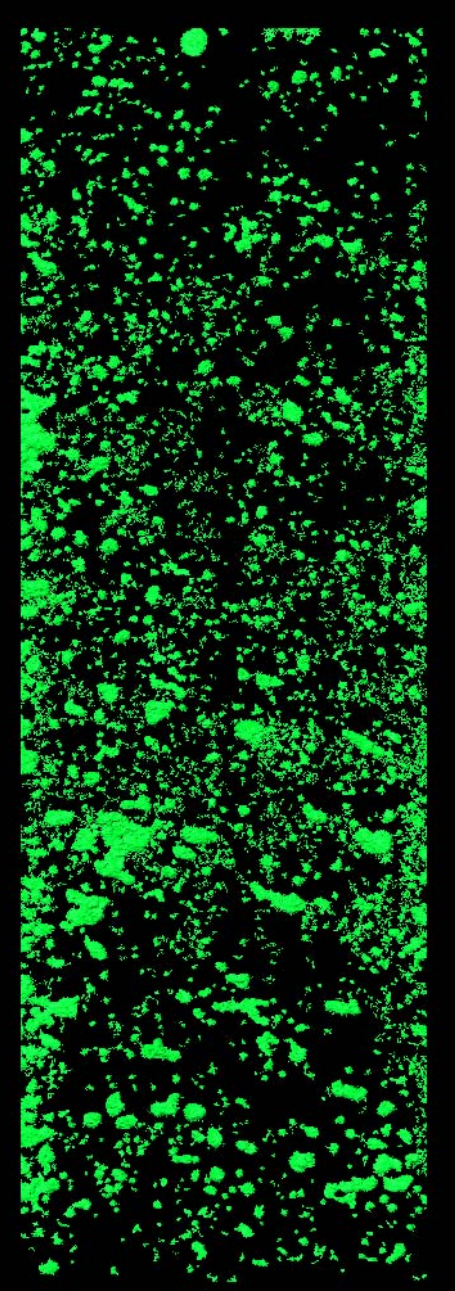

c

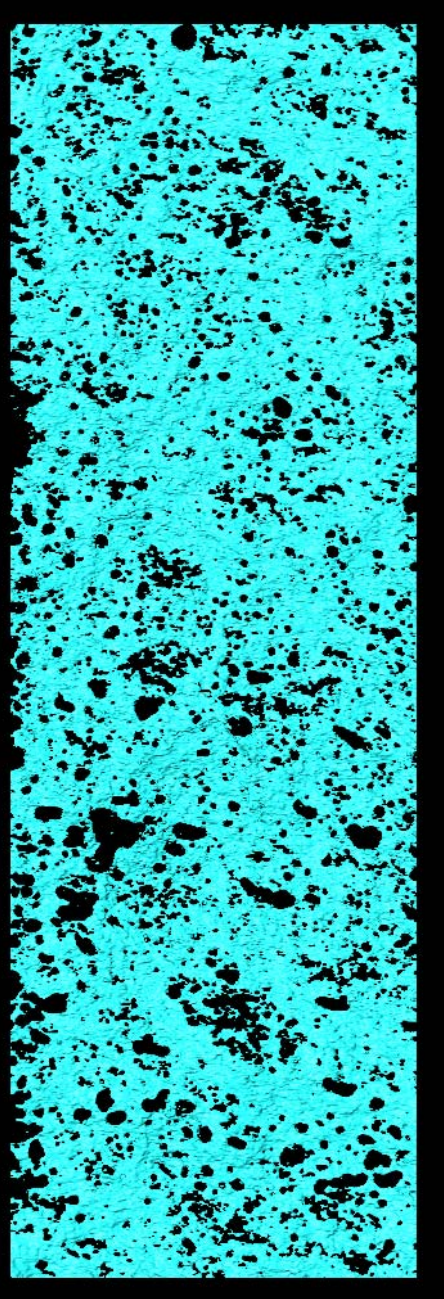

Figure 105: Fluids distribution maps: a: dry. b: Residual oil. c: Water 
Fractional Flow: After reaching residual oil saturation, different fractional flow injection sequences were done in order to establish possible trends. Figure 106 shows the pressure drop against fractional flow diagrams. The first path of fractional flow was done starting at fractional flow (water) equal 1.0 moving towards fractional flow equal zero. The total flow rate of $12 \mathrm{cc} / \mathrm{min}$ was used for the first flow test. The four traverses of the fractional flow range exhibit increase in pressure drop per a fixes $f_{w}$ value. This indicates a strong dependency of the conductivity of the fracture on drainage or imbibition process and a changing saturation distribution with time.

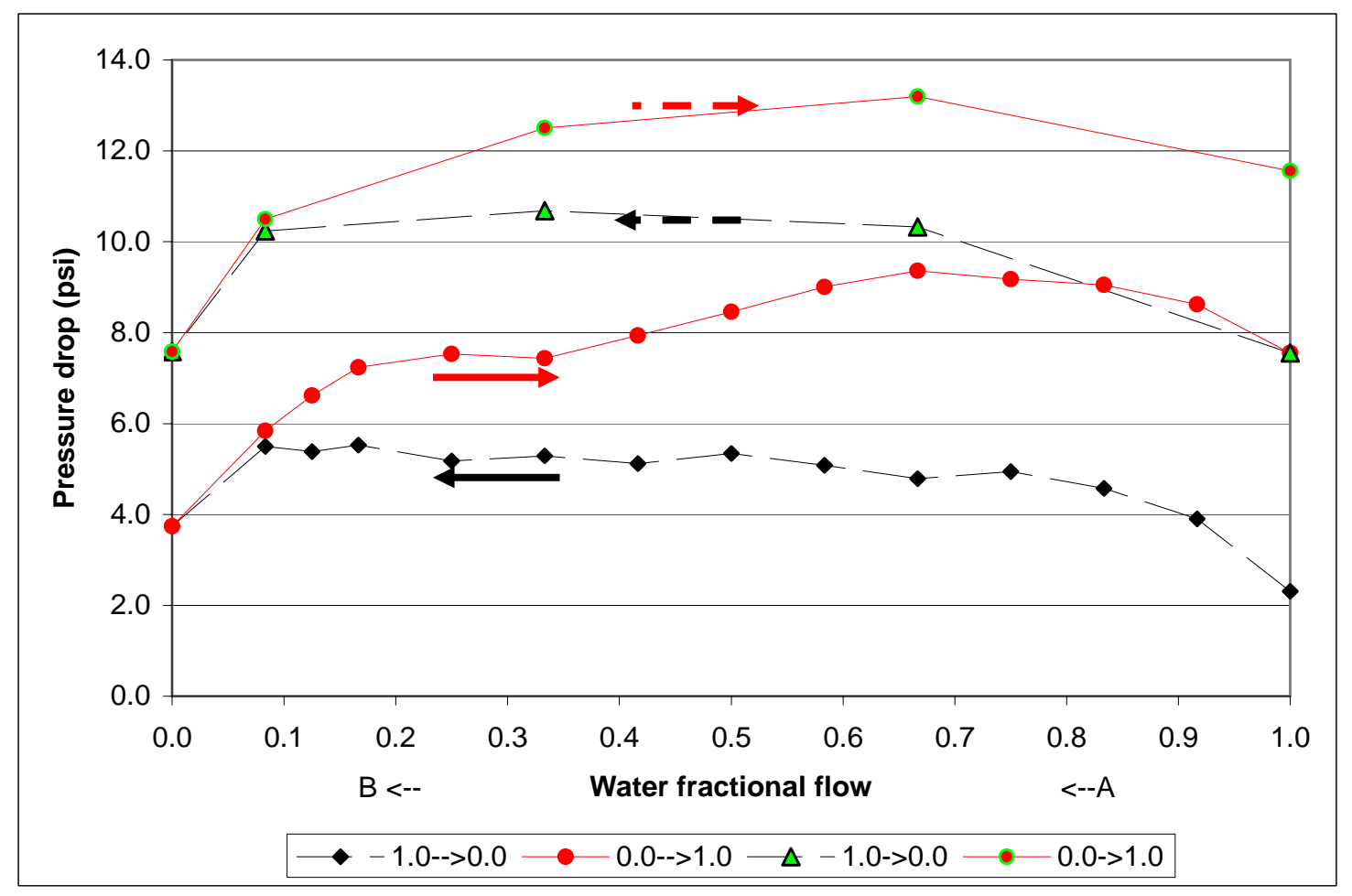

Figure 106: Pressure drop as function of fractional flow: two drainage and imbibitions cycles. 
Figure 107 shows different loops where the flow injection was stopped as consequence of the high pressure drop. The maximum allowable pressure drop was 18 psig. A build up in pressure drop in the fracture was consecutively observed for different fractional flows (bottom to top). However, when the flow was reversed (top-bottom) the pressure drop in the fracture dropped to its original value. When the flow direction was reestablished (bottom-top) a new build up was produced. Scans of the injection and production ends showed that there was no buildup of water or oil and that the end connectors were not responsible for the cyclic nature of the pressure drop along the fracture. The entire fracture was scanned at low pressure drop and high pressure drop in order to find the fluids saturation under both conditions. Figure 108 shows in a three-dimensional reconstruction of the oil and water in the fracture for the low pressure drop condition.

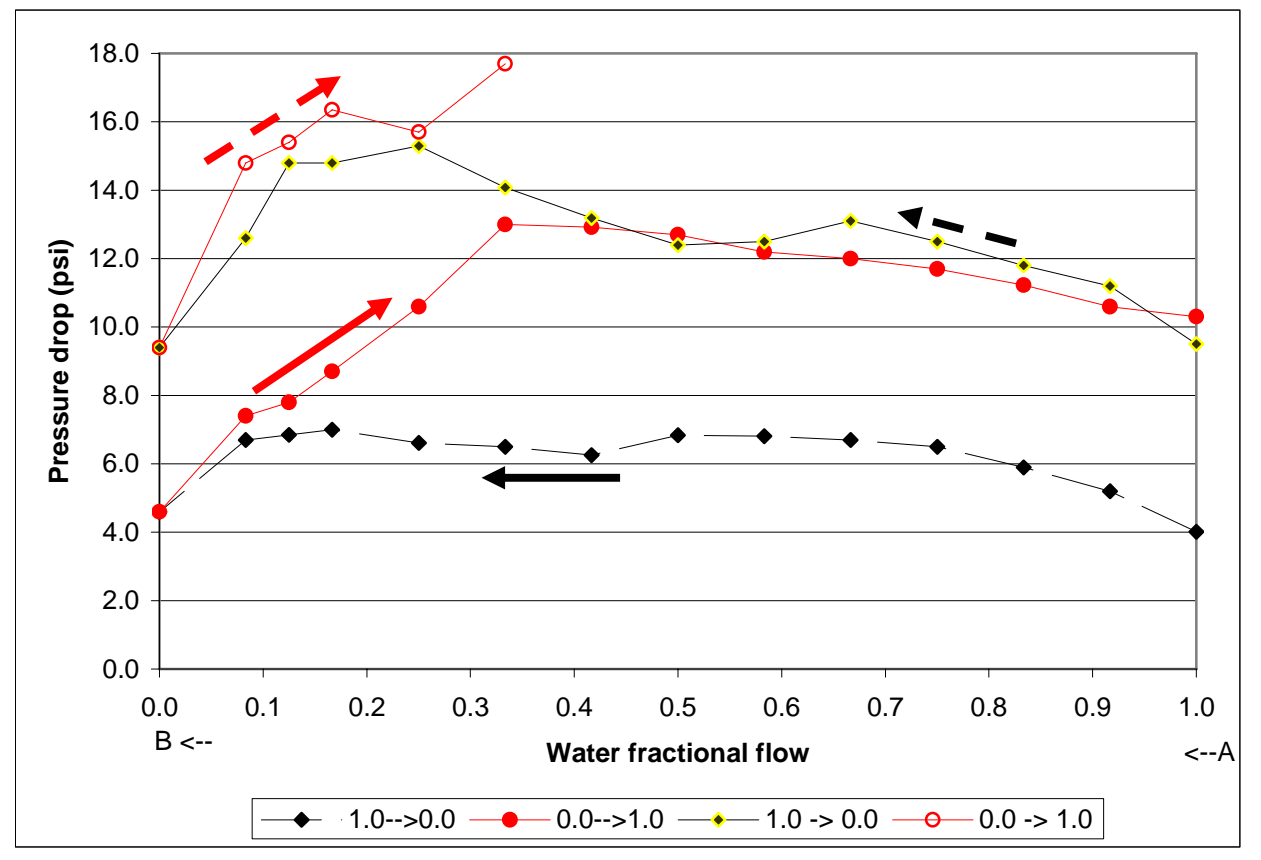

Figure 107: Pressure drop in the fracture for different fractional flow. $\mathrm{q}_{\mathrm{total}}=12 \mathrm{cc} / \mathrm{mim}$. 


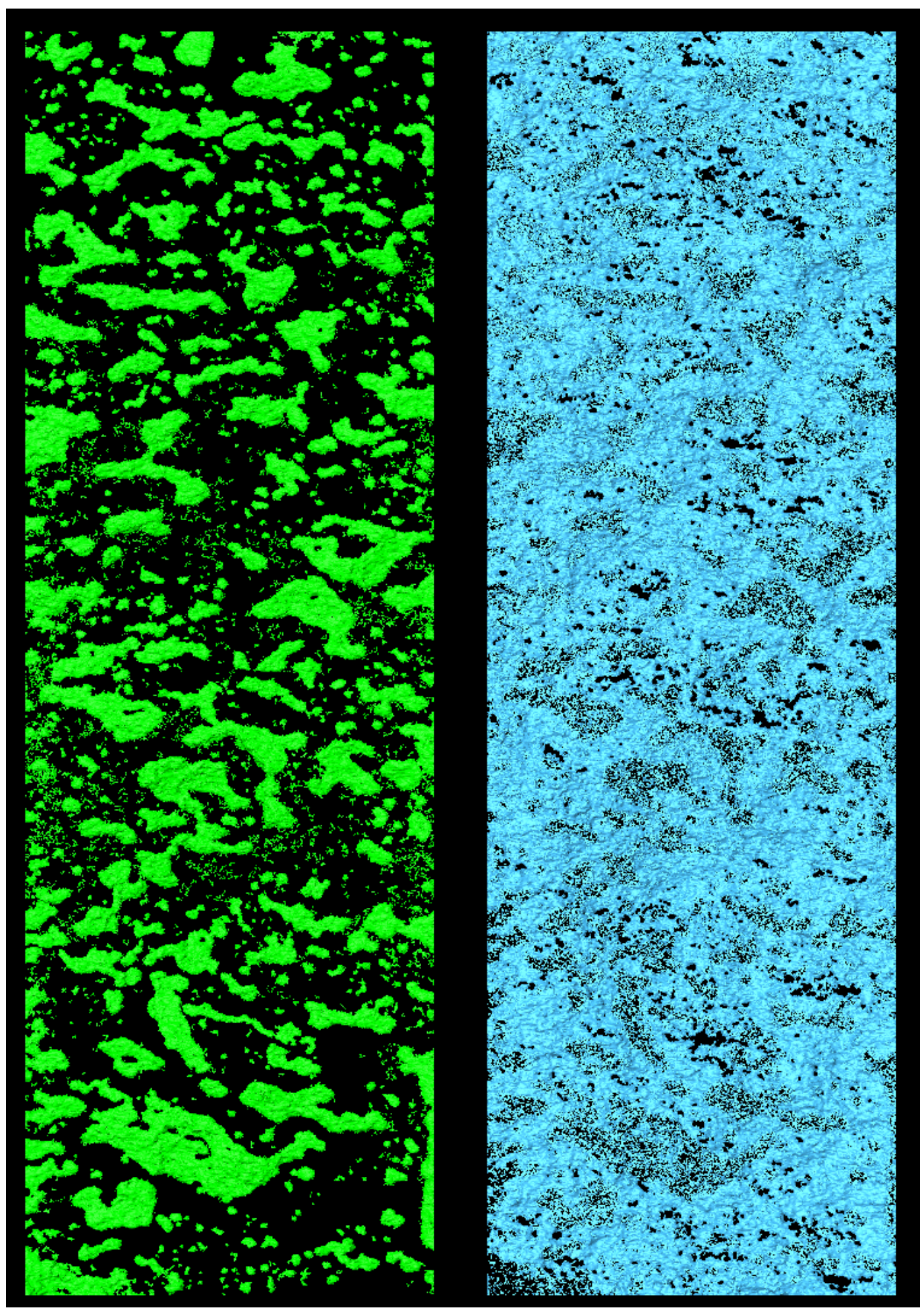

Figure 108: Fluids distribution in the fracture at low pressure drop. a: Oil b: water. 
The over all fluid saturations were $38.6\left(\mathrm{~S}_{\mathrm{of}}\right)$ and $61.4 \%\left(\mathrm{~S}_{\mathrm{wf}}\right)$ for oil and water respectively. An average saturation profile along the fracture is shown in Figure 109. The total volume of oil in the system for the low pressure drop was $145 \mathrm{~mm}^{3}$. The minimum and maximum oil saturation (cross sectional) values in the fracture were $13.1 \%$ and 63.3\%, respectively. Different non-connected oil regions are shown in Figure 110, where it is also highlighted the largest oil globule with a value of $9.3 \mathrm{~mm}^{3}$. 

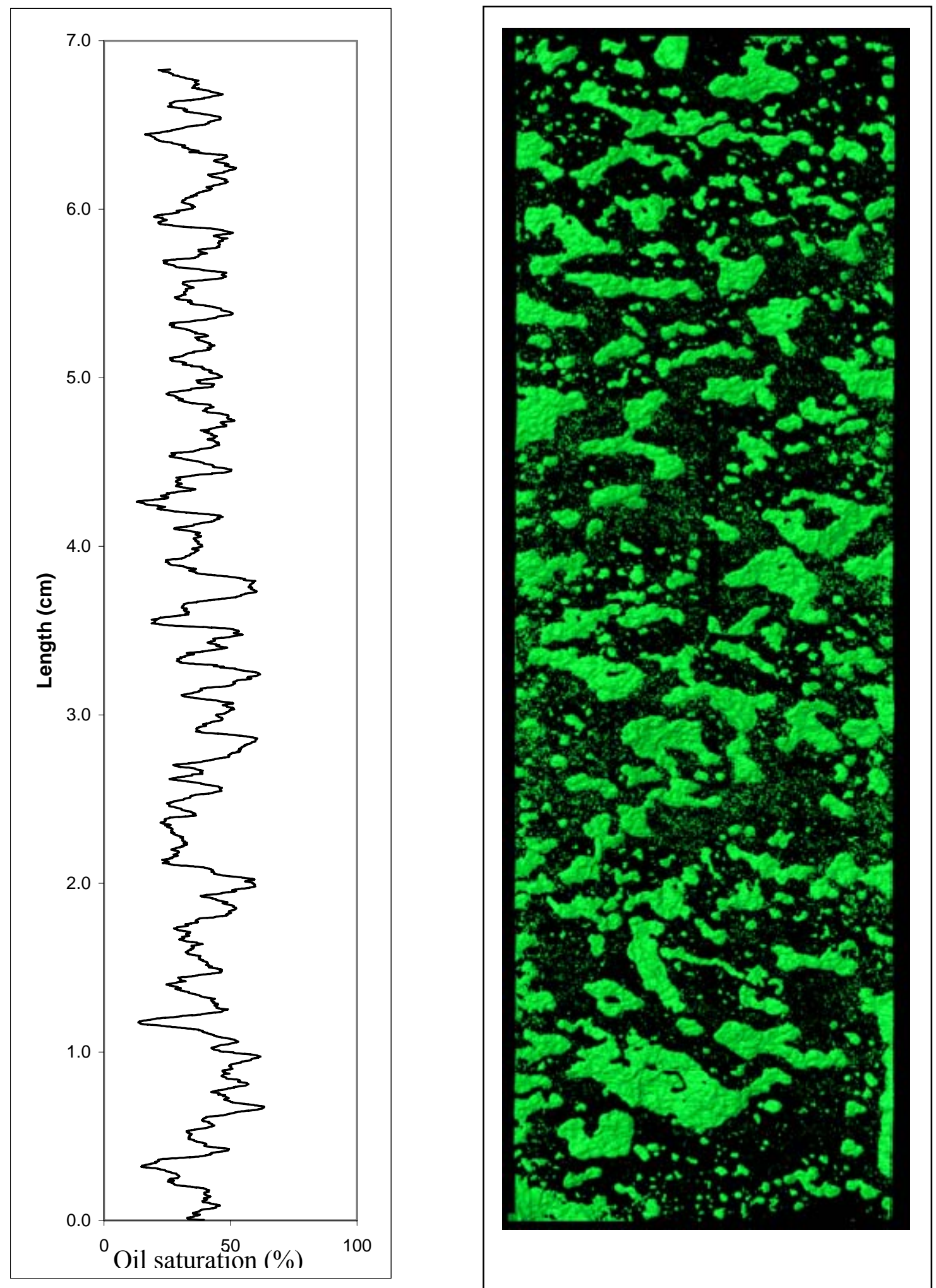

Figure 109: Oil saturation profile for the low pressure drop condition (left).Three-dimensional distribution of oil in the fracture (right). 


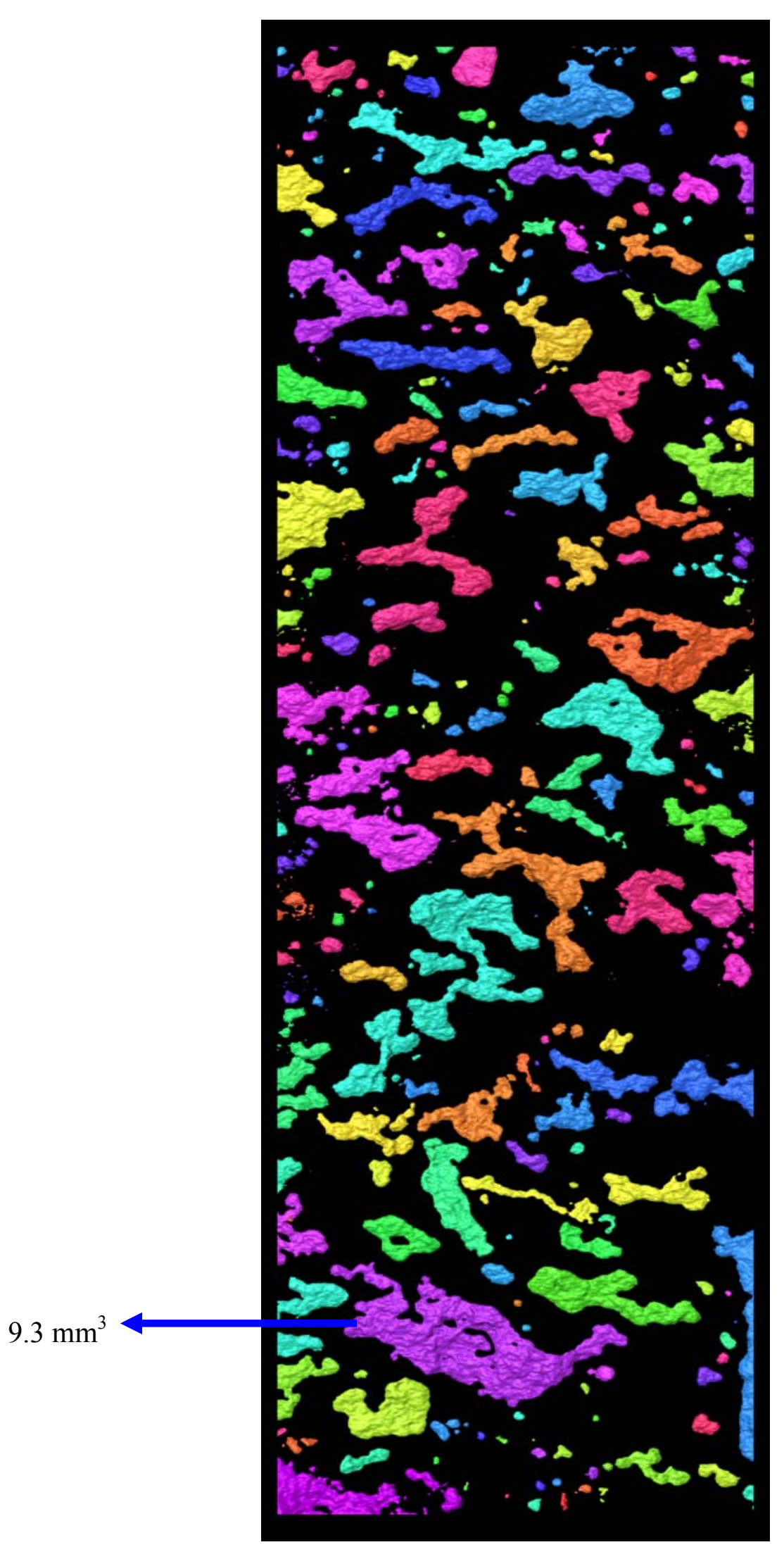

Figure 110: Disconnected oil globules in the fracture at low pressure drop condition. 
A total of 240 independent oil bodies were identified. Figure 111 presents the distribution of the oil bodies, where the left part of the graph is affected by the limitation of the x-ray system to detect small volumes at the current resolution (26 microns). Most of the oil ganglias fall in the range between 0.01 and $0.09 \mathrm{~mm}^{3}$.

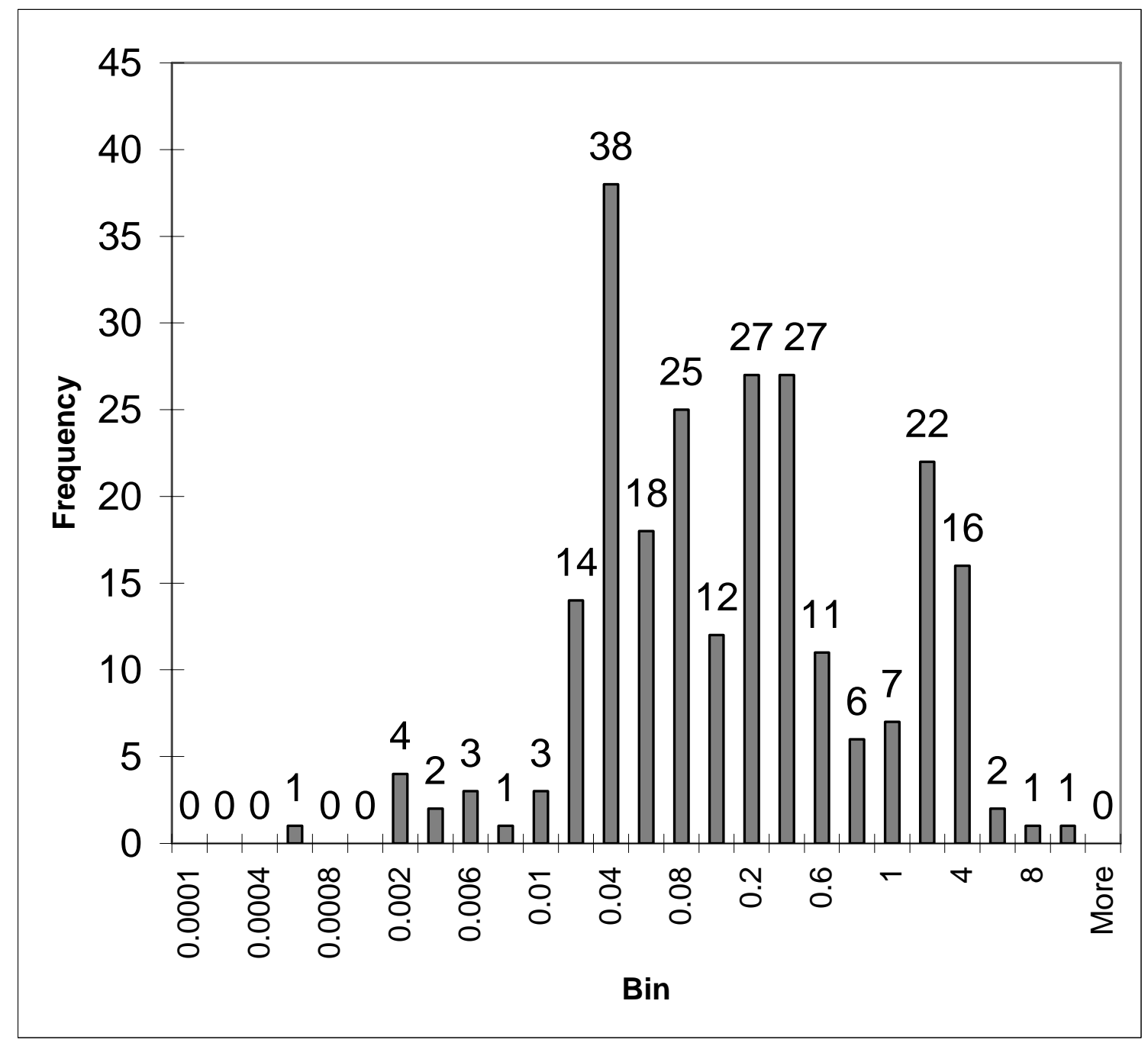

Figure 111: Oil bodies distribution at low pressure drop. 
The saturation structure during the two-phase flow was not always stable. Figure 112 shows a sudden change in pressure drop that occurred at the end of an imbibition cycle during a steady injection. This significant loose in pressure drop could be produced by the displacement of oil globules (snap-off) in the fracture, and it was not possible to document it given that the $\mathrm{CT}$ time acquisition is longer than time in which this event occurred.

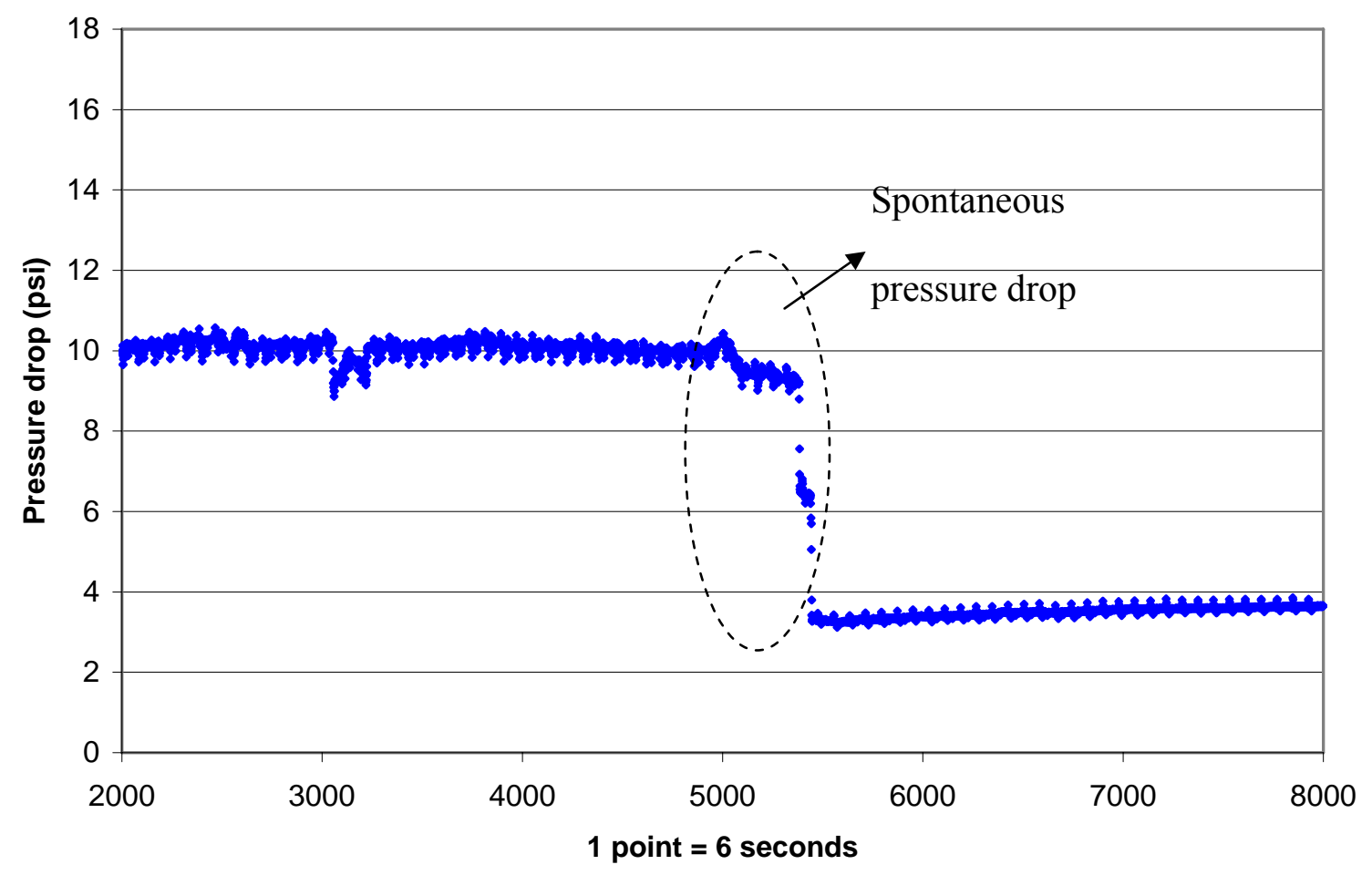

Figure 112: Fracture pressure drop during different fractional flows including a spontaneous pressure change. 
Figure 113 shows the saturation profile (left) for the fracture at high pressure drop after different fractional flow cycles. The right part of Figure 113 shows a three dimensional image of how the oil is distributed in the fracture. The volume of oil in the fracture is equal to $0.142 \mathrm{cc}$, and is similar to the value computed in the low pressure drop system $(0.145 \mathrm{cc})$.

The over all computed fluid saturation were $37.8\left(\mathrm{~S}_{\mathrm{of}}\right)$ and $62.2 \%\left(\mathrm{~S}_{\mathrm{wf}}\right)$ for oil and water respectively. Table 13 shows a comparison of the fracture saturation for both cases, low and high pressure drop. The volumes of oil and water in the fracture are similar. However, there are few regions where the non-wetting phase (oil) seems to have a higher local saturation. 

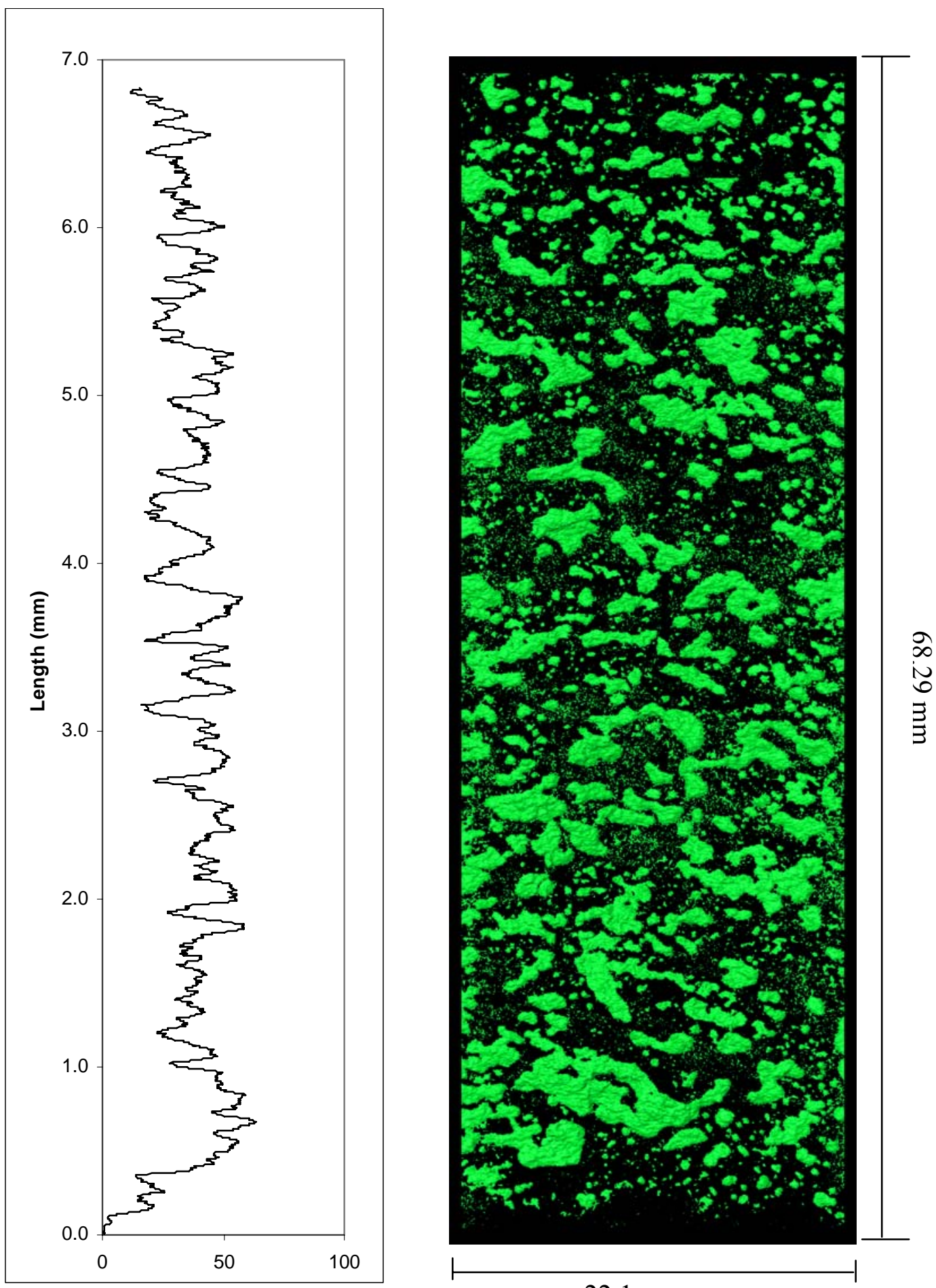

o
ஸ
3
3
3

Figure 113: Oil saturation profile for the high pressure drop condition (left).Threedimensional distribution of oil in the fracture (right). 
Table 13: Fluid fracture saturation comparison at high and low pressure

\begin{tabular}{|l|c|c|}
\hline \multicolumn{1}{|c|}{ Pressure drop $\Delta \mathrm{p}$} & Sof (\%) & Swf (\%) \\
\hline Low & 38.6 & 61.4 \\
\hline Hi & 37.8 & 62.2 \\
\hline
\end{tabular}

Figure 114 shows the large oil globule at the two pressure drop conditions. This figure shows that although the overall saturation is about the same (Table 12) the distribution of the fluids is significantly different. The red ellipse highlights a specific region for comparison. The yellow connected oil globule on the left (high pressure drop) is much smaller in the low pressure drop case. This "yellow" globule impedes fluid flow and increases the pressure drop along the fracture.

Figure 115 shows a section, between 2 and $3 \mathrm{~cm}$ (axially) along the fracture where the oil saturation of the high pressure drop case is significant higher than the low pressure drop case. 


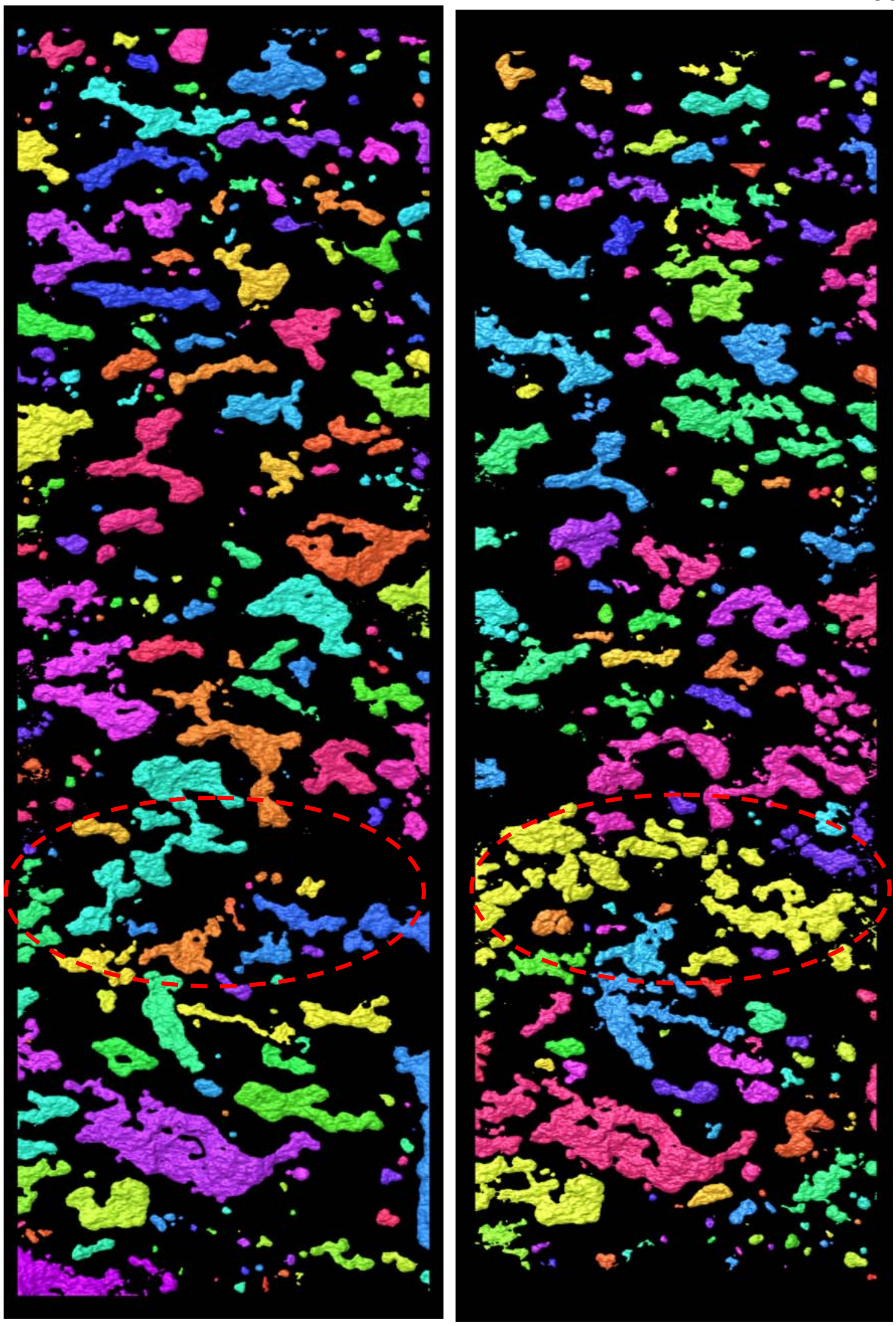

Figure 114: Oil saturation profile for the high pressure drop condition (left).Threedimensional distribution of oil in the fracture (right). 
a: Low pressure drop $\quad$ b: High pressure drop

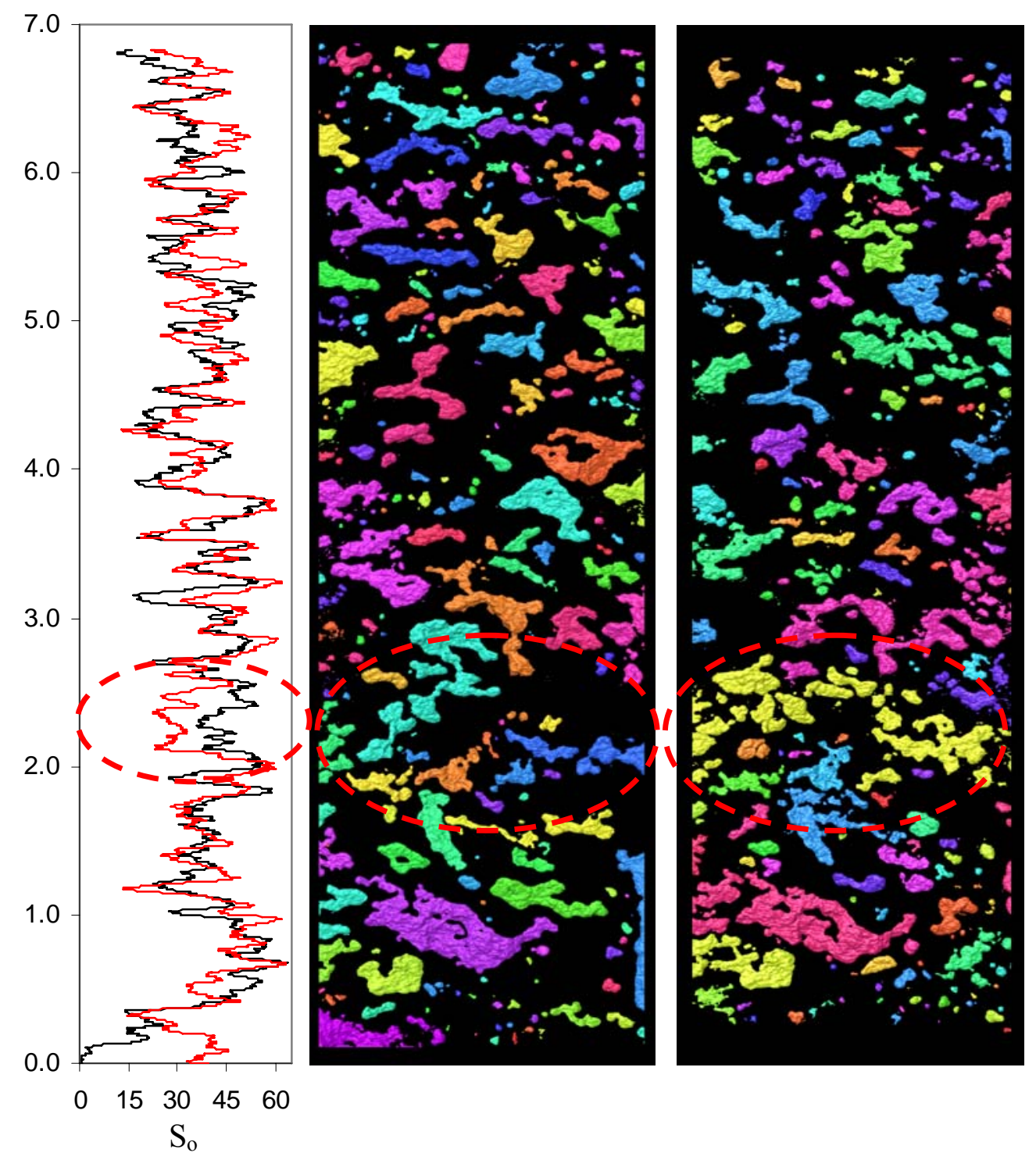

Oil saturation for the high pressure drop case.

Oil saturation for the low pressure drop case.

Figure 115: Oil distribution low pressure drop (a). Oil distribution high pressure drop (b). 
The previous figures demonstrate that the over all fluid saturations in the fracture are almost identical for high and low pressure drop. However, it is the distribution of the non wetting phase (oil) that creates a difference in the pressure drop. The low conductivity of flow in the fracture forces the fluids to flow through the matrix, thus, increasing the pressure drop of the system. In order to analyze and compare the fracture conductivity over the Berea sandstone a non-fractured "neighbor" core was used to inject fluids and compare the pressure drops. Figure 116 shows the pressure drop for a fracture and a unfracture core for different flow rates. A significant pressure drop in the presence of residual oil shows the impact of the non weatting phase.

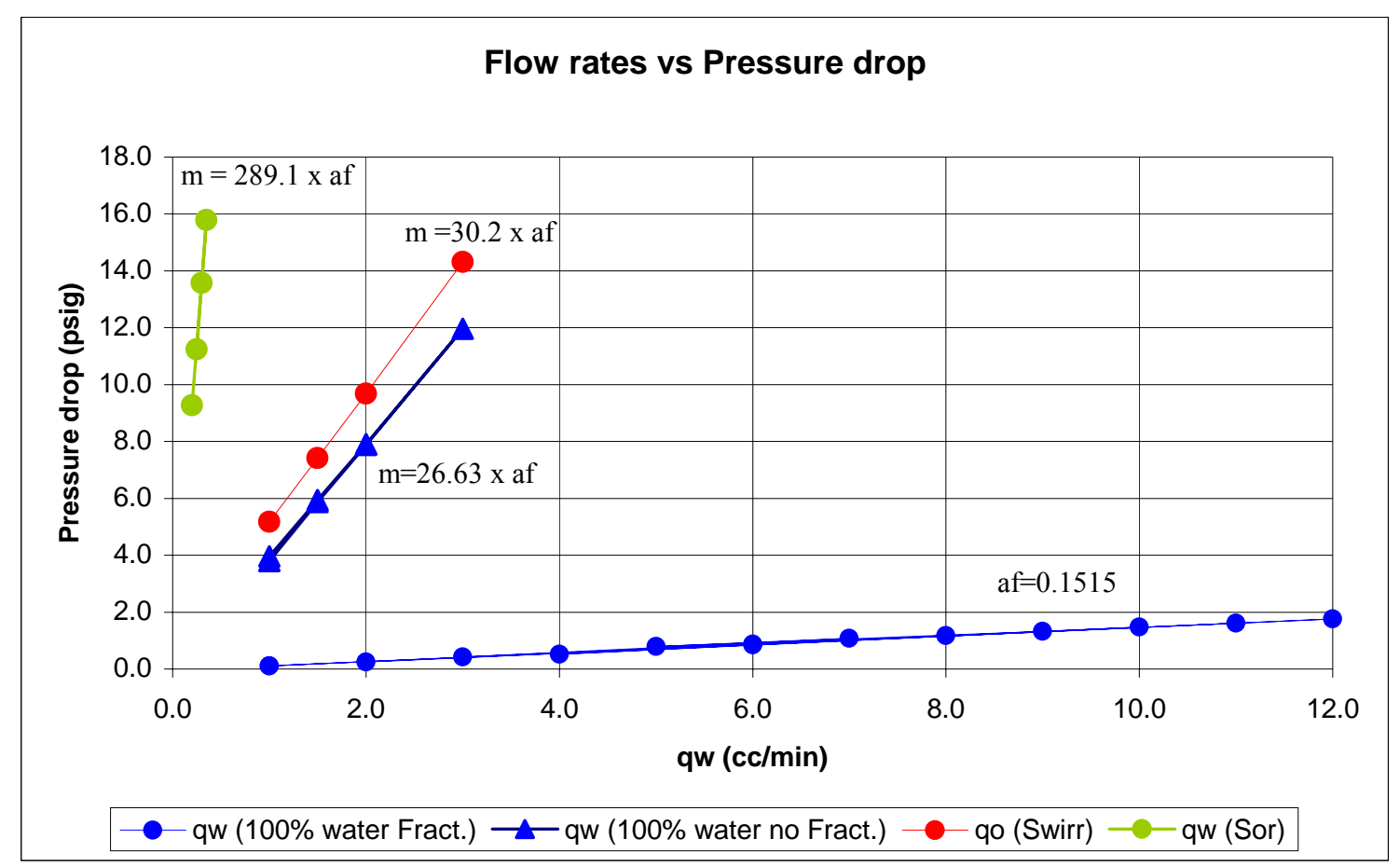

Figure 116: Pressure drop as a function of flow rate for a non-fractured and a fractured core. 
Reversing the flow direction in the fracture allows the fluids to rearrange and release the pressure buildup. This fracture plugging behavior can be directly related to problems with gas wells, where field data report possible water slugs plugging wells in hydraulically fractured gas reservoirs, Christiansen et al. (2005). Some of the field solutions for re-establishing the flow for these type of wells have been injecting from the top of the wells (reversing flow). 
Confining pressure from 500 to 2500 psig: The sample was at a saturation condition created by steady water and oil injection prior to increasing the confining pressure. During the increase in confining pressure the system was at low water flow rate $(0.3$ cc/min). Figure 117 shows a schematic of the fracture system after changing the confining pressure from 500 to 2500 psig.

The volume of the fracture prior to the change in confining was $375.8 \mathrm{~mm}$ and, $280.4 \mathrm{~mm}$ after increasing the confining pressure. Table 14 shows the fracture and fluids volume for the two different confining pressures, before and after changing the confining stress.

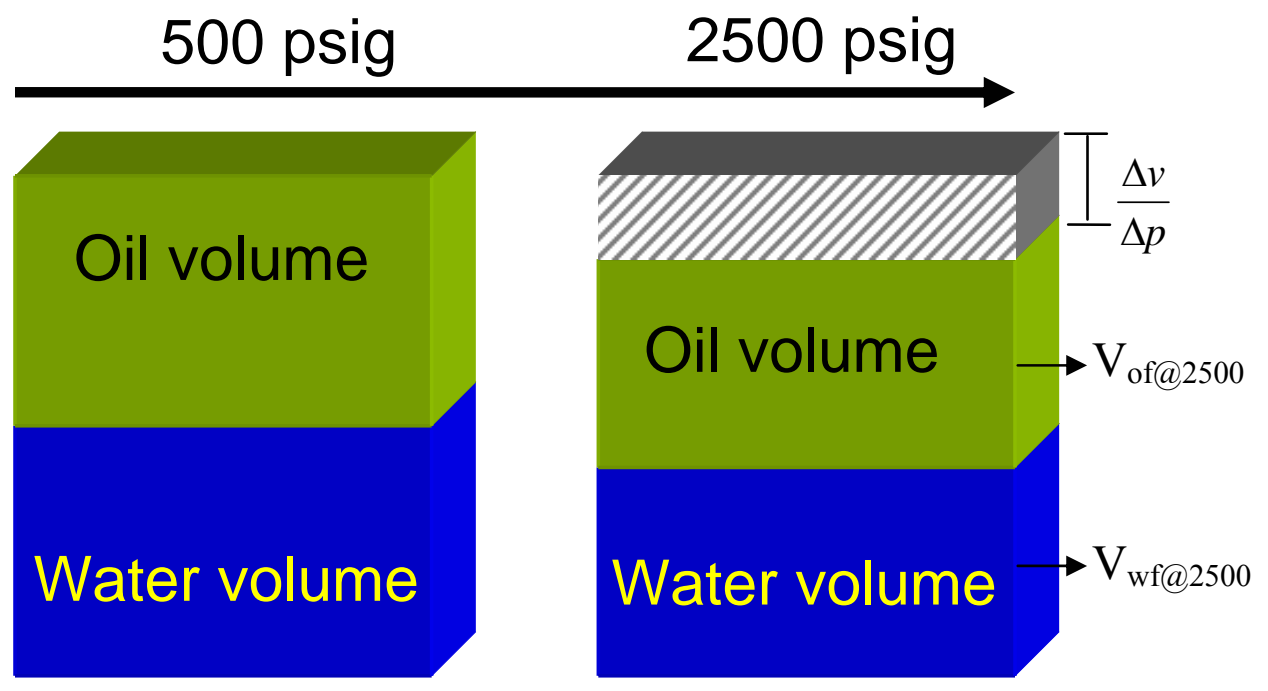

Figure 117: Schematic of the volume changes with confining pressure. 
The oil saturation decreased by about $6 \%$ indicating a preferential removal of oil from the fracture, though both oil and water were removed (oil volume removed: $52.5 \mathrm{~mm}^{3}$, water volume removed: $43.1 \mathrm{~mm}^{3)}$.

Table 14: Volume change with confining pressure. Before and after $P_{c}$ changes

\begin{tabular}{|c|c|c|c|c|c|}
\hline $\begin{array}{c}\text { Confining } \\
\text { pressure } \\
(\mathrm{psig})\end{array}$ & $\begin{array}{c}\text { Fracture } \\
\text { volume } \\
\left(\mathrm{mm}^{3}\right)\end{array}$ & Oil Volume & $\begin{array}{c}\text { Water } \\
\text { Volume } \\
\left(\mathrm{mm}^{3}\right)\end{array}$ & Saturation & Water \\
\hline 500 & 375.8 & 142.4 & 233.5 & 37.9 & 62.1 \\
\hline 2500 & 280.4 & 89.9 & 190.4 & 32.1 & 67.9 \\
\hline
\end{tabular}

The increase in confining pressure reduced the volume of the fracture and changed the volume of the water and oil present in the fracture. The volume of fluids that disappeared from the fracture could either flow out of the system through the ends of the fracture or flow into the matrix.

Average CT profiles from the matrix adjacent to the fracture are presented in Figures 118 and 119. In Figure 119 CT profiles for the dry and wet conditions are shown to highlight the significant difference in $\mathrm{CT}$ values. 
Figure 119 highlights the similar CT registration of the matrix at 500 and 2500 psig confining pressure, indicating that migration of fluids from the fracture to the matrix did not occur. A material balance would be useful to corroborate the production of the fluids from the fracture. However, given the small changes in volume and the magnitude of the dead volume such a balance calculation is not viable.

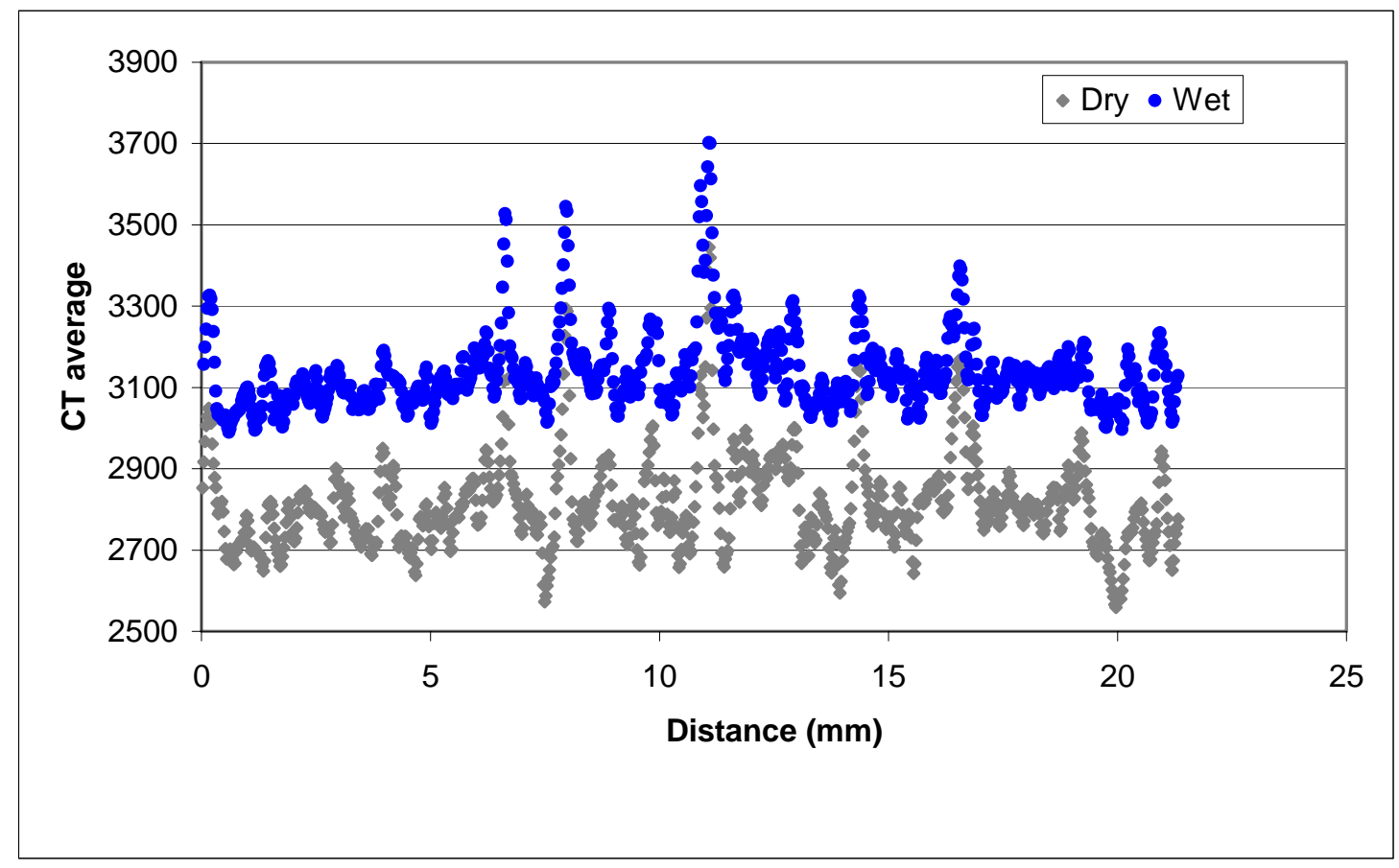

Figure 118: CT profile in the matrix area near the fracture (Dry and Wet). 


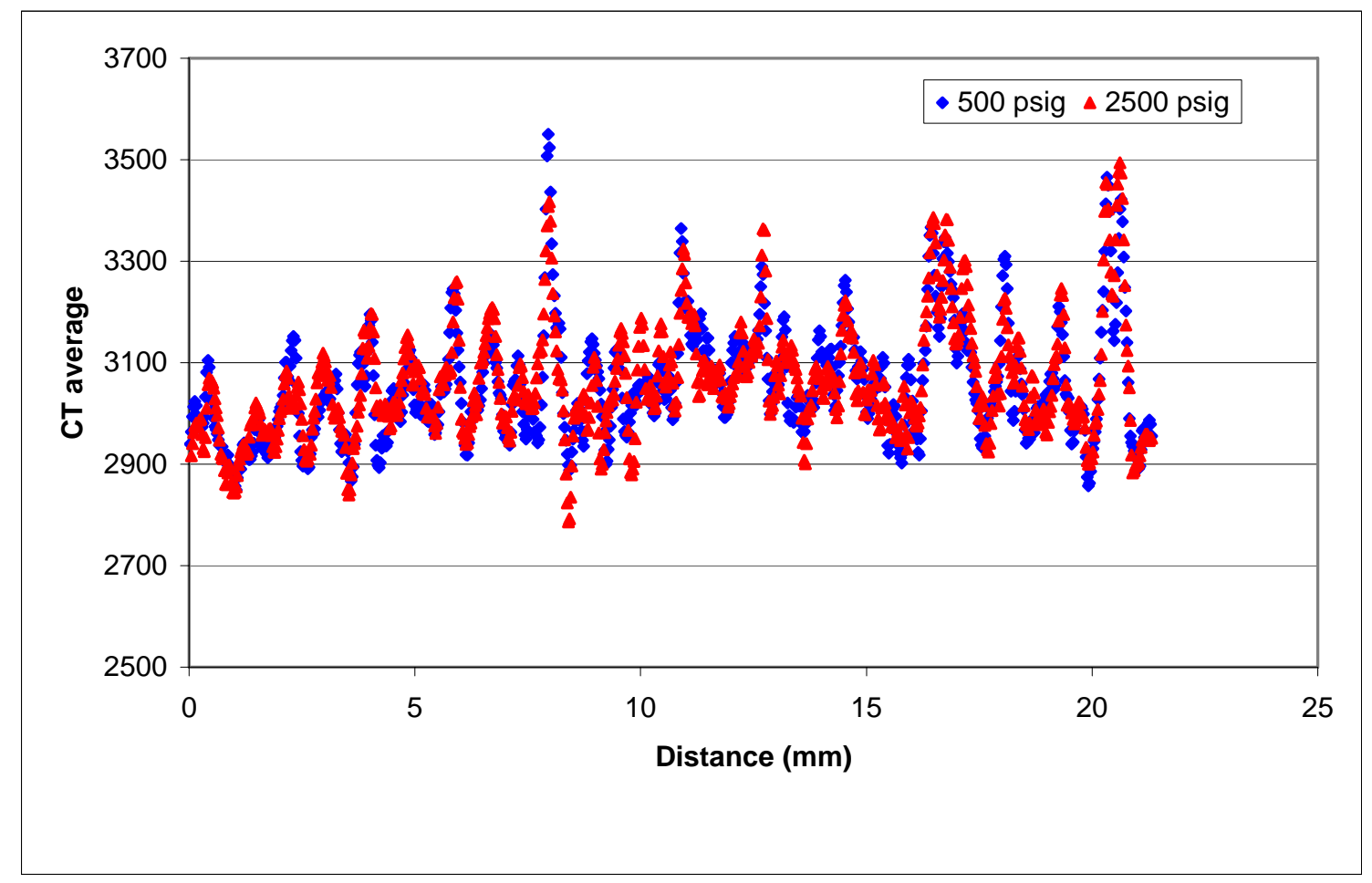

Figure 119: $C T$ profile in the matrix area near the fracture at 500 and 2500 psig.

After increasing the confining pressure from 500 to 2500 psig a new state of residual oil saturation was reached and the system was scanned at this condition. Figure 120 shows the three dimensional distribution of the residual oil saturation in the fracture at 2500 psig. The fracture volume was $280.4 \mathrm{~mm}^{3}$. 
Table 15 shows the values of the fluids saturation before and after the changes in confining pressure. The oil saturation in the fracture was $41.3 \%$ at 2500 psig, more than double the saturation of the oil saturation at $500 \mathrm{psig}(18.6 \%)$.

Table 15: Fracture fluids saturation before and after changes in Pc

\begin{tabular}{|c|c|c|}
\hline Confining Pressure (psig) & $\mathrm{S}_{\mathrm{of}}(\%)$ & $\mathrm{S}_{\mathrm{wf}}(\%)$ \\
\hline 500 & 18.6 & 81.4 \\
\hline 2500 & 41.3 & 58.7 \\
\hline
\end{tabular}

Table 15 highlights the residual oil saturation for both changes in confining pressure, 500 and 2500 psig. The oil mobile range reduced in $22 \%$ from 500 to 2500 psig confining pressure. This significant reduction in mobile oil saturation in the fracture is a key observation that has a major effect on the recovery oil from the fracture.

Table 15: Residual oil saturation and mobile at 500 and 2500 psig

\begin{tabular}{|c|c|c|}
\hline Confining Pressure (psig) & $\mathrm{S}_{\text {orf }}(\%)$ & Mobile Oil (\%) \\
\hline 500 & 18.6 & 70 \\
\hline 2500 & 41.3 & 48 \\
\hline
\end{tabular}




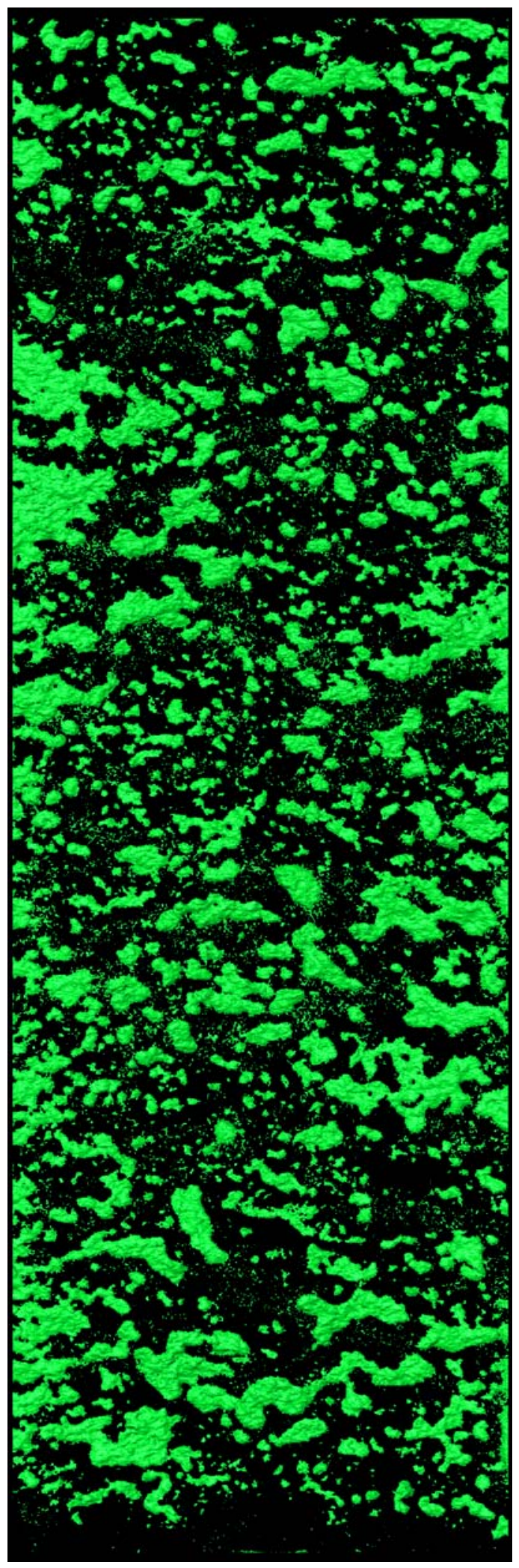

Figure 120: Residual oil saturation map at 2500 psig. 
Different fractional flow injections were performed at 2500 psig, showing similar behavior to the responses seen prior to increasing the confining pressure. Figure 121 shows the pressure drop along the fracture as function of fractional flow of water at 2500 psig. The imbibition curve ended above the drainage curve indicating changes in the internal distribution of the phases.

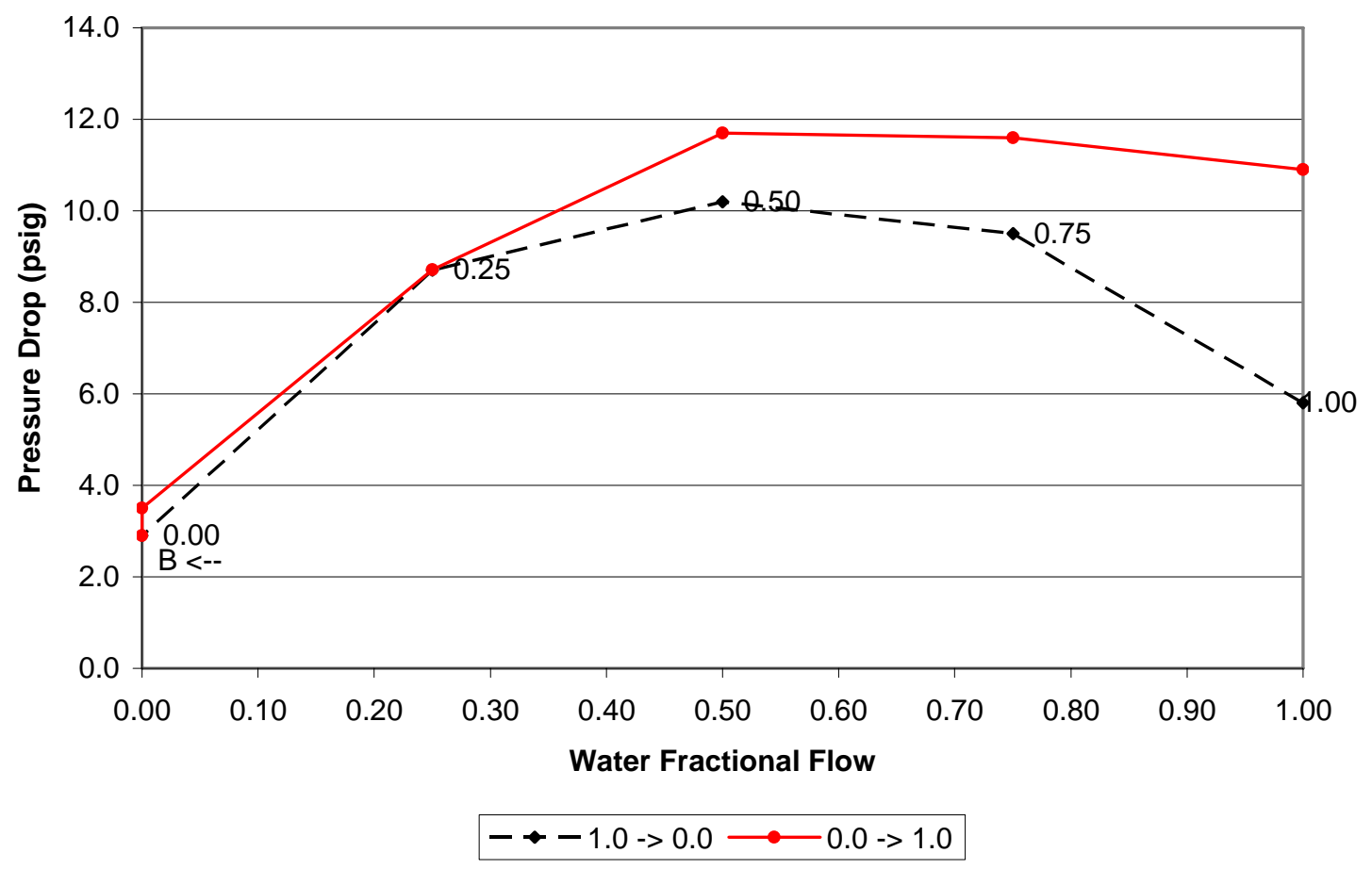

Figure 121: Pressure drop in the fracture at 2500 psig for a total liquid flow rate of $1 \mathrm{cc} / \mathrm{min}$. 
The isolation of the fracture at the first confining pressure state (500 psig) was done by extracting the digital fracture from the dry scans. Since the sample was not moved during the experiment, excellent $\mathrm{CT}$ registration was obtained and the virtual fracture from the dry scans was used to isolate the fracture in the other condition. The situation at the high confining pressure (2500 psig) was different as the sample was saturated. To obtain the dry scans the sample was cleaned by injecting Ethyl Alcohol (dozen of pores volumes). Figure 122 shows the changes of CT values at a fixed position as a function of time. When the $\mathrm{CT}$ values stabilized the sample was displaced by nitrogen and then vacuum dried. Once dry conditions were established the sample was scanned and the virtual fracture at a confining pressure of 2500 psig was extracted.

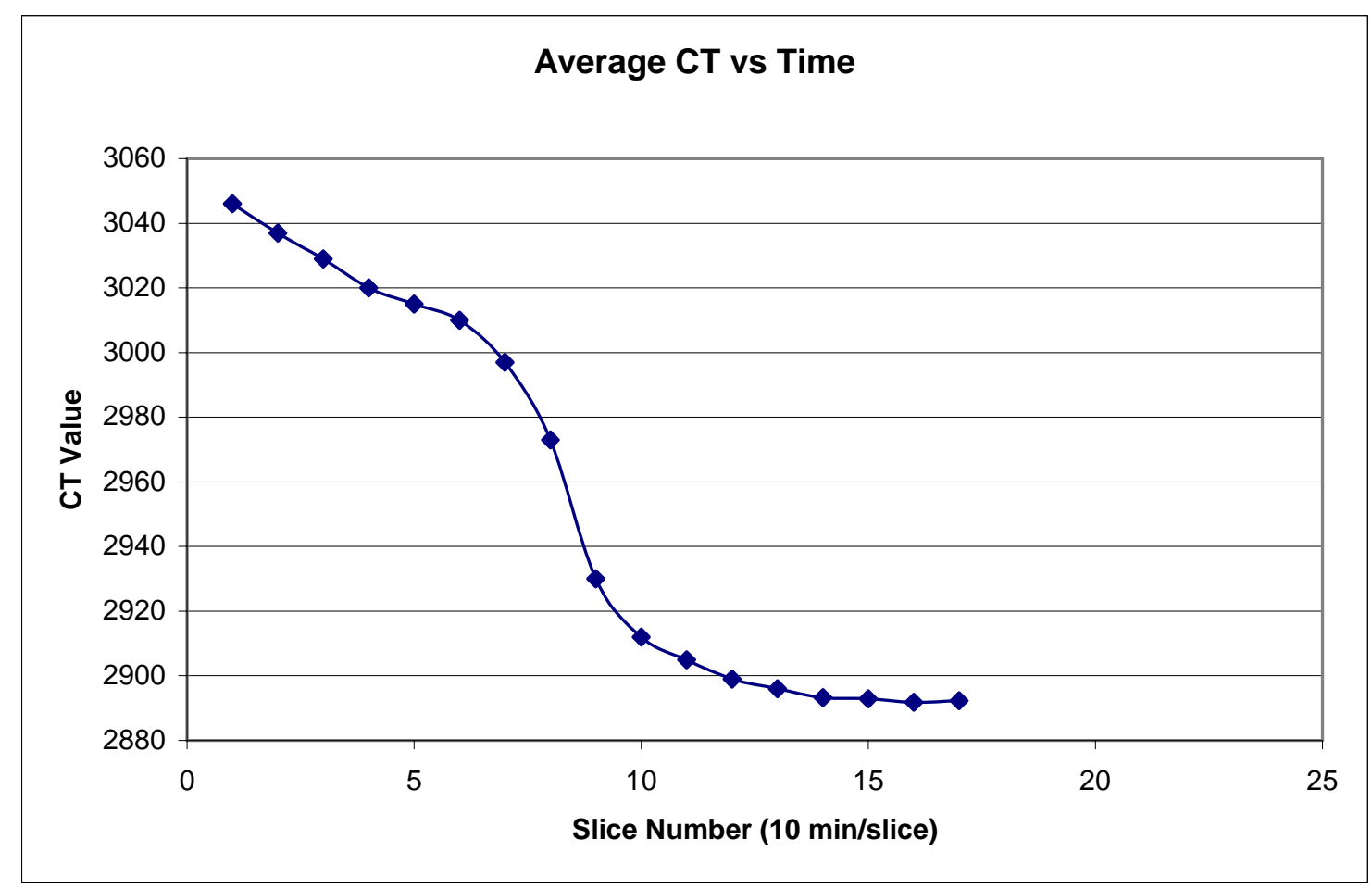

Figure 122: Changes in CT average (2500 psig) after injecting alcohol and vacuum drying of sample. 
The fracture volume for the fracture at 2500 psig was computed showing a reduction in volume from 375.8 to $280.4 \mathrm{~mm}^{3}$. Figure 123 shows three-dimensional renditions of the fracture at 500 and 2500 psig at dry condition demonstrating a significant increase of asperities. An aperture fracture thickness map for both cases is shown in Figure 124 highlighting regions with conductivity (bright areas), while the red color denotes the asperities. A total of $2.9 \%$ of the aperture thickness map corresponds to asperities for the case of 500 psig, while $11.9 \%$ corresponds to the case were the confining pressure was raised to 2500 psig. This core shows a much higher change in the percentage of asperities $(9 \%)$ than previous cases (Core BS1), where only $4.1 \%$ changes in asperities area for the same change in confining pressure. 


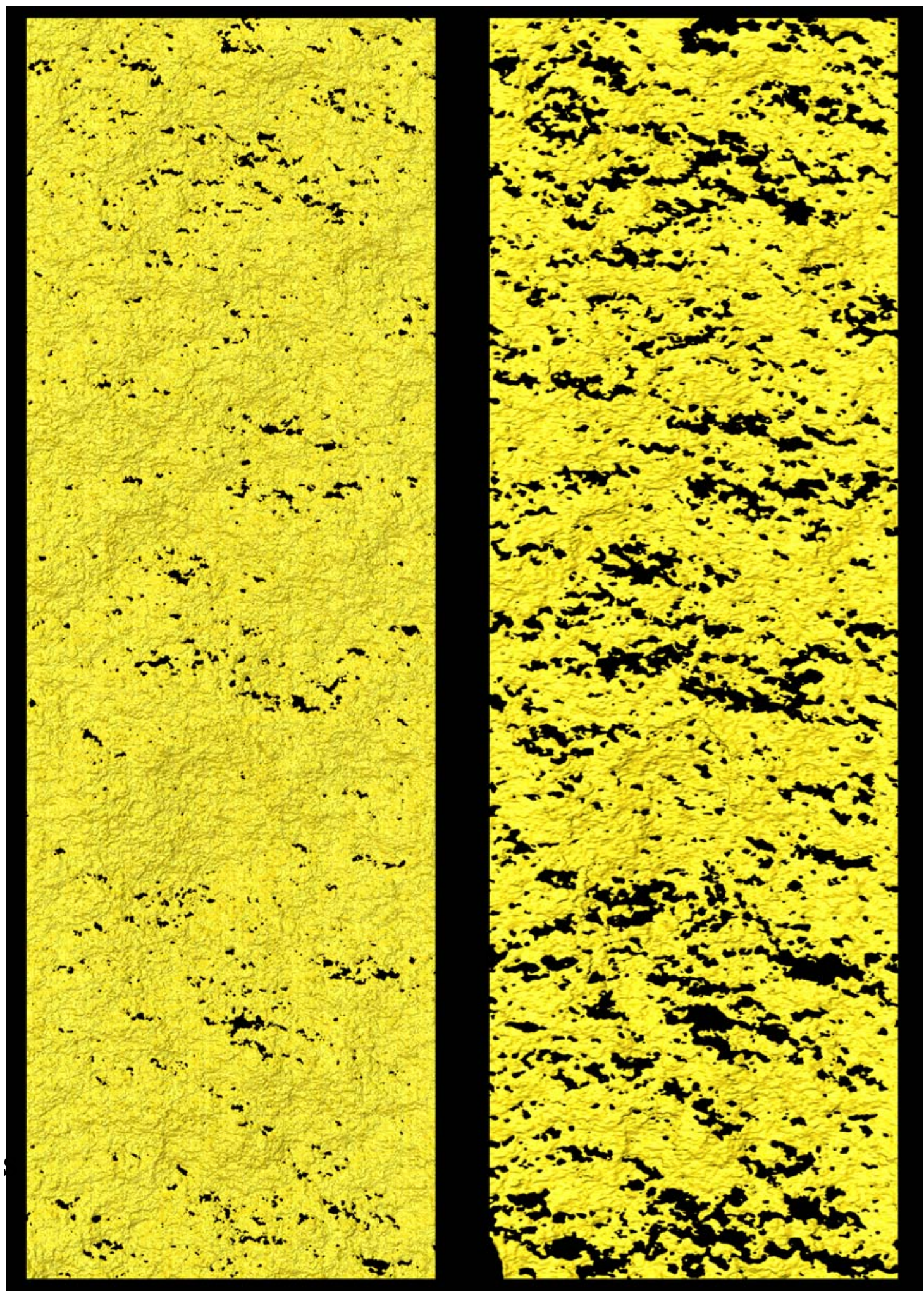

Figure 123: Three-dimensional recreation of the fracture at 500 (left) and 


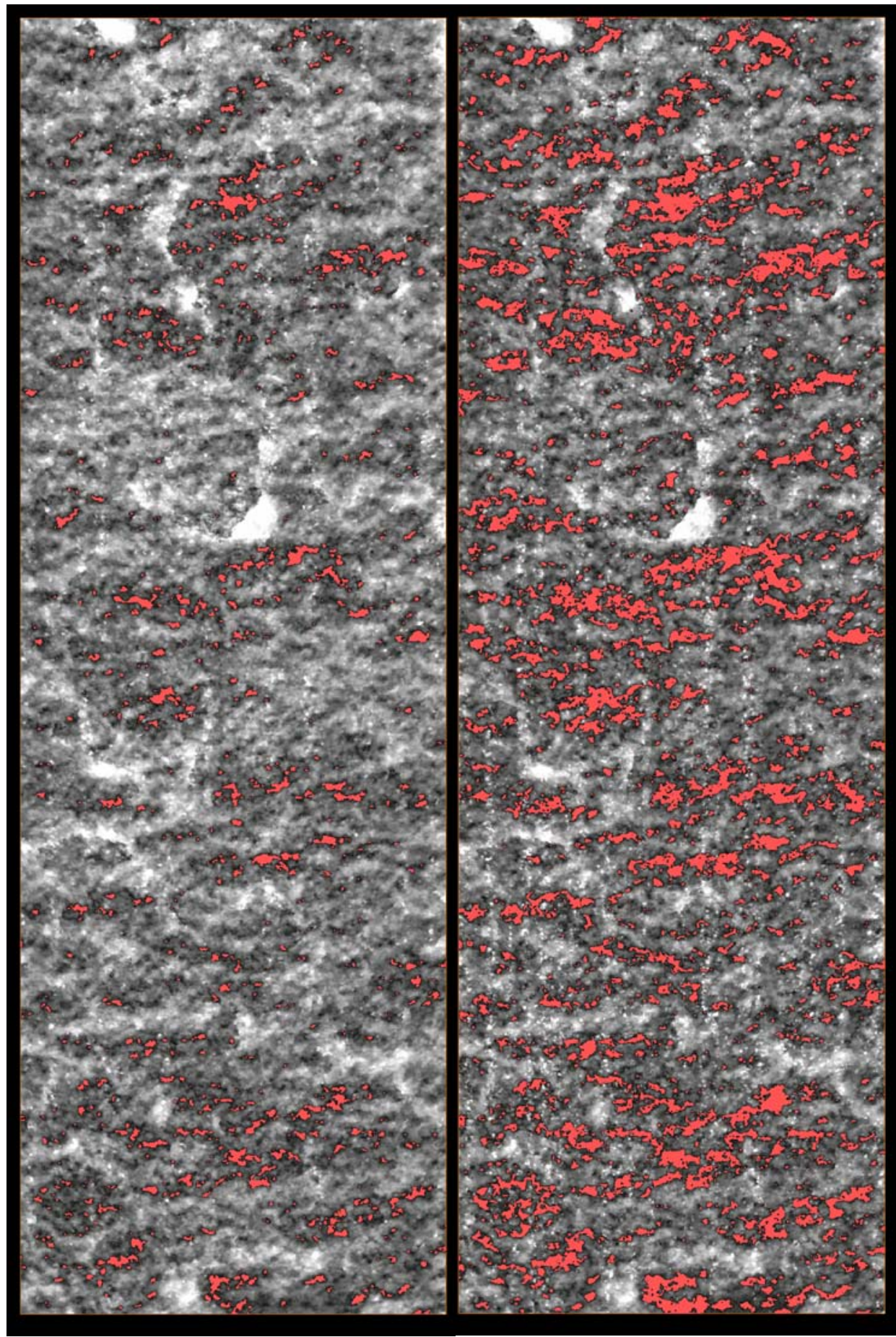

Figure 124: Thickness aperture map of the fracture at 500 (left) and 2500 (right) psig. 
Summary: A study of the behavior of multiphase flow in a tensile fracture has been performed. This study includes the characterization and the interaction of the fluids during mechanical changes in the tensile fracture using MCT. The EPC and SMI methods were used to demonstrate the fracture structure response to changes in confining stress. These characterizations will be significant in our ability to classify different fractures based on their topology. The distribution of the oil and water along the fracture have a significant impact on pressure drop and therefore on fluid transport and recovery. The change in fracture aperture has a significant impact on the fracture pore space and therefore on fluid occupancy. The redistribution of the phases in the fracture, as a consequence of changing confining stress, controls the multiphase transport properties of the fracture. The following observations and conclusions are made:

1. The distribution of the phases along the fracture modifies the conductivity of the fracture. The non wetting phase produces plugging regions that drastically reduce the multiphase conductivity of the fracture. This fracture plugging behavior can be directly related to problems with gas wells, where field data report possible water slugs plugging wells in hydraulically fractured gas reservoirs.

2. The use of the cubic law for flow through fractures severely over estimates the permeability of the fractures studied in this research. The conductivities were much lower than the predicted by the cubic law. 
3. In the cases studied, relative permeabilities deviated significantly from the $\mathrm{x}-$ shaped curves typically used for fractures systems. In particular the conventional assumption grossly over predicts $\mathrm{K}_{\mathrm{ro}}$. Application of these observations should greatly improve accuracy of dual-permeability simulators.

4. As expected, increasing the confining pressure reduced fracture aperture and absolute permeability. Aperture closure correlates with reduction in absolute permeability. A general increase in asperity contact area accompanied the observed aperture reduction.

5. The increase in confining stress caused significant reduction in the residual oil saturation in the fracture. This reduction in residual oil saturation $\left(\mathrm{S}_{\mathrm{or}}\right)$ has a major effect on the change of relative permeabilities and on the mobile saturation range of a fracture. The residual water saturation $\left(S_{\text {wirr }}\right)$ remained constant at the pressure ranges used in this experiment.

6. Flow instability of the pressure drop during drainage and imbibition cycles was observed.

7. At constant fracture fractional flow a continuous decrease in the fracture conductivity was observed. This reduction in conductivity was not stable and was removed by reversing the flow. X-rays CT confirmed the hypothesis that 
instability is caused by change in phase distribution in the fracture with little change in saturation.

8. A methodology for computing a deterministic coefficient for measuring the connectivity density, EPC, (connections per unit volume) and the fracture structure model index, SMI, of a fractured system was presented. In the future, we expect to correlate multiphase hydraulic properties of the fracture and these topological coefficients. 


\section{CONCLUSIONS}

The conclusion and summary statements are attached to each chapter in the report.

\section{REFERENCES}

The references included here are the inclusive list from all the semi-annual reports and for the theses derived from this project.

Alajmi, A. F. and Grader, A. S. (2000). Analysis of Fracture-Matrix Interactions using XRay ct. In Proceedings of the SPE Eastern Regional Meeting, volume 123, pages 97104. Society of Petroleum Engineers.

Alvarado, F. E., Grader, A. S., Karacan, O. and Halleck, P. M.: "Visualization of Three Phases in Porous Media Using Micro Computed Tomography," Petrophisycs 45, 2004.

Babadagli, T. and Ershaghi, I.: "Imbibition Assisted Two-Phase Flow in Natural Fractures," SPE, 1992.

Barton, N., Bandis, S., and Bakhtar, K. (1985). Strength Deformation and Conductivity Coupling of Rock Joints. International Journal of Rock Mechanics and Mining Sciences \& Geomechanics Abstracts, 22(3), 121-140.

Chen, D. W., Zimmerman, R. W. and Cook, N. G. W.: "The effect of contact area on the permeability of fractures," 30. US symposium on rock mechanics, Morgantown, WV (USA), 1989.

Christiansen, R., Mayoral, C. and Pereira, C.: "Capillary End Effects and Gas Production From Low Permeability Formation," SCA, Canada, 2005.

Churchel, P. L., P. B. French, J. C. Shaw, and L. L. Schramm, Rock Properties of Berea Sandstone, Baker Dolomite and Indiana Limestone, Proceedings of the 1991 International Symposium on Oilfield Chemistry, vol. 68, pp 431-446, February 1999.

Durham, W. B., Bourcier, W. L. and Burton, E. A.: "Direct observation of reactive flow in a single fracture," Water Resources Research 37 (1): 1-12, 2001.

Engelder, J. T., Cataclasis and the Generation of Fault Gouge, Geological Society of America Bulletin, vol. 85, pp 1515-1522, 1974.

Esaki, T., Du, S., Mitani, Y., Ikusada, K., and Jing, L. (1999). Development of shear flow test apparatus and determination of coupled properties for a single rock joint. 
International Journal of Rock Mechanics and Mining Sciences, vol. 36,pages 641650.

Gentier, S., Lamontangne, E., Archambault, G., and Riss, J. (1997). Anisotropy of Flow in Fracture Undergoing Shear and its Relationship to the Direction of Shearing and Injection Pressure. International Journal of Rock Mechanics and Mining Sciences Vol. 34 No. (3-4), page 412.

Gentier, S., Riss, J., Archambault, G., Flamand, R., and Hopkins, D. (2000). Influence of fracture geometry on shear behaviour. International Journal of Rock Mechanics and Mining Sciences, Vol. 37, pages 161-174.

Grader, A. S., Balzarini, M., Radaelli, F., Capasso, G., and Pellegrino, A., FractureMatrix Flow: Quantification and Visualization Using X-ray Computerized Tomography. AGU Monograph Volume 122, October 2000.

Gale, J. E.: "Comparison of coupled fracture deformation and fluid flow models with direct measurement of fracture poro-structure and stress-flow properties," Symposium of Rock Mechanics, 1987.

Grader, A. S. and O'Meara, D. J., Jr.: "Dynamic Displacement Measurements of ThreePhase Relative Permeabilities Using Three Immiscible Liquids," SPE Annual Meeting, Huston, Texas, 1998.

Gray, H. D., Fatt, I. and Bergamini, G.: "The Effect of Stress on Permeability of Sandstone Cores," SPEJ: 95-100, 1963.

Hicks, P. J., Jr., Deans, H. A. and Narayama, K.: "Distribution of Residual Oil Saturation on Heterogeneous Carbonate Cores Using X-Ray CT," SPE: 235-239, 1992.

Hildebrand, T. and Ruegsegger, P.: "Quantification of Bone Microarchitecture with the Structure Model Index," Computer Methods in Biomechanics and Biomedical Engineering 1: 15-23, 1997.

Horie, T., Firoozabadi, A. and Ishimoto, K.: "Laboratory Studies of Capillary Interaction in Fracture/Matrix Systems," SPE, 1990.

Iwai, k., Fundamental Studies of Fluid Flow Through a Single Fracture, PhD thesis, University of California, Berkley, 1976,

Jamison,W. R., and L. W. Teufel, Pore Volume Changes Associated With Failure and Frictional Sliding of a Porous Sandstone, 20th US Symposium on Rock Mechanics, pp 167-170, June 1979. 
Karpyn, Zuleima (2005). Capillary-Driven Flow in Fractured Sandstone. PhD. Thesis, Penn State University.

Keller, A. (1998). High Resolution, Non-destructive Measurement and Characterization of Fracture Apertures. International Journal of Rock Mechanics and Mining Sciences, Vol. 35, No. 8, pages 1037-1050.

Keller, A. A.: "Single and Multiphase Flow and Transport in Fractured Porous Media," Stanford, California, Stanford University, 1996.

Khilar, K. C. and Fogler, H. S.: "Colloidally induced fine migration in porous media," Reviews in chemical engineering No. 4: 41-108, 1987.

Lomize, G. M.: "Flow in Fractured Rocks" (in Russian). Gosenergoizdat, Moscow: 127, 1951.

Louis, C.: "A study of groundwater flow in jointed rock and its influence on the stability of rock masses," Rock Mech. Res Rep. 10: 90, 1969.

Makurat, A. (1985). The Effect of Shear Displacement on the Permeability of Natural Rough Joints. In Proceedings of the 17th International Congress of the International Association of Hydrogeologists, Vol. 39, pages 99-106.

Mohammad, Nazia (2004). Effects of Artificial Shear Fracture on Two-Phase Flow in Berea Sandstone. Ms. Thesis, Penn State University,

Montemagno, C. D., Pyrak-Notle, L. J. (1999). Fracture Network Versus Single Fractures: Measurement of Fracture Geometry with X-ray Tomography. Phys. Chem. Earth (A), Vol. 24, No. 7, pages 575-579.

Neuzil, C. E. and Tracy, J. V.: "Flow Through Fractures," Water Resources Research 17: 191-199, 1981.

Odgaard, A. and Gundersen, H.: "Quantification of Connectivity in Cancellous Bone, with Special Emphasis on 3-D Reconstructions," Bone 14: 173-182, 1993.

Olsson, W. A., Brown, S. R., (1993). Hydromechanical Response of A fracture Undergoing Compression and Shear. International Journal of Rock Mechanics and Mining Sciences, Geomech. Apstr. 30, 845-851.

Pepper, J. F., Jr. W. Dewitt, and D. F. Demarest, Geology of Bedford Shale and Berea Sandstone in Appalachian Basin, United States Geological Survey - Professional Papers, vol. 259, pp 109, 1954. 
Persoff, P. and Pruess, K.: "Two-Phase Flow Visualization and Relative Permeability Measurements in Natural Rough-Walled Rock Fractures," Water Resources Research 31 (5), 1985.

Pyrak-Nolte, L. J., Montemagno, C. D. and Nolte, D. D.: "Volumetric Imaging of aperture distribution in connected fracture networks," Geophysical Research Letters 24:18: 2343-2346, 1997.

Pyrak-Nolte, L. J., Myer, L. R., Cook, N. G. W. and Witherspoon, P. A.: "Hydraulic and mechanical properties of natural fractures in low permeability rock," Proceedings of the 26th International Congress of Rock Mechanics, 1987.

Pyrak-Nolte, L. J., Nolte, D. D., Myer, L. R. and Cook, N. G. W.: "Fluid flow through fractures," Proceedings of the International Symposium on Rock Joints, Norway, 1990.

Raven, K. G., and J. E. Gale, Water Flow in a Natural Rock Fracture as a function of Stress and Sample Size, International Journal of Rock Mechanics and Mining Sciences \& Geomechanics Abstratcs, vol. 22.4, pp 251-261, August 1985

Revil, A. Pervasive pressure solution in a quartz sand. J. Geophys. Res., 106, B5, 86658686, 2001.

Schrauf, T. W. and Evans, D. D.: "Laboratory Studies of Gas Flow Through a Single Fracture," Water Resources Research 22 (7): 1038-1050, 1986.

Teufel, L. W. (1987). Permeability Changes During Shear Deformation of Fractured Rock. In Proceedings of the 28th U.S. Symposium on Rock Mechanics, volume 129, pages $473-480$.

Tsang, Y. W.: "The effect of tortuosity on fluid flow through a single fracture," Water Resources Research 20 (9): 1209-1215, 1984.

Tsang, Y. W. and Tsang, C. F.: "Channel Model of Flow Through Fractured Media," Water Resources Research 23 (3): 467-479, 1987.

Vinegar, H. J., and Wellington, S. L., Tomographic Imaging of Three-Phase Flow Experiments, Rev. Sci. Insts., January, pp. 96-107, 1987.

Vukuturi, V. S., Lama, R. D., and Saluja, S. S., Handbook on Mechanics Properties of Rocks, Volume I Trans Tech Publications, 1974.

Walsh, J. B.: "The effect of pore pressure and confining pressure on fracture permeability," Int. R. Mech. Min. Sci. \& Geomech. 18: 429-435, 1981. 
Walter, D. A. and Wong, R. C. K.: "The hydraulic and mechanical response if an oil sand fracture under a varying pressure," Can. Geotech. J 36: 262-271, 1999.

Wang, S. Y., Ayral, S. and Gryte, C. C.: "Computed Tomography for the Observation of Oil Displacement in Porous Media," SPE: 53-55, 1984.

Wellington, S. L. and Vinegar, H. J.: "X-Ray Computerized Tomography," Journal of Petroleum Technology: 885-887, 1987.

Witherspoon, P. A., Wang, J. S. Y., Iwai, K. and Gale, J. E.: "Validity of cubic law for fluid flow in a deformable rock fracture," Water Resources Research 16: 1016-1024, 1980.

Yeo, I. W., Freitas, M. H. and Zimmerman, R. W.: "Effect of shear displacement on the aperture and permeability of a rock fracture," Int. J. Rock. Mech. Min. Sci. Geomech. 35: 1051-1070, 1998.

Zimmerman, R. W., Kumar, S. and Bodvarsson, G. S.: "Lubrication theory analysis of the permeability of rough-walled fractures," International Journal of Rock Mechanics and Mining Science and Geomechanical Abstracts 28(B12): 325-331, 1991.

\section{LIST OF ACRONYMS AND ABBREVIATIONS}

